\title{
Inorganic Fullerene-like Nanoparticles and Inorganic Nanotubes
}

\section{Edited by}

Reshef Tenne and Andrey N. Enyashin

Printed Edition of the Special Issue Published in Inorganics 
Reshef Tenne and Andrey N. Enyashin (Eds.)

\section{Inorganic Fullerene-like Nanoparticles and Inorganic Nanotubes}


This book is a reprint of the special issue that appeared in the online open access journal Inorganics (ISSN 2304-6740) in 2014 (available at:

http://www.mdpi.com/journal/inorganics/special_issues/inorganic_fullerenes).

\section{Guest Editors}

Reshef Tenne

Weizmann Institute

Israel

Andrey N. Enyashin

Institute of Solid State Chemistry UB RAS

Russia

Editorial Office

MDPI AG

Klybeckstrasse 64

Basel, Switzerland

Publisher

Shu-Kun Lin

Managing Editor

$\mathrm{Min} \mathrm{Su}$

\section{Edition 2015}

MDPI • Basel • Beijing • Wuhan

ISBN 978-3-03842-149-8 (PDF)

ISBN 978-3-03842-150-4 (Hbk)

(C) 2015 by the authors; licensee MDPI, Basel, Switzerland. All articles in this volume are Open Access distributed under the Creative Commons Attribution 4.0 license (http://creativecommons.org/licenses/by/4.0/), which allows users to download, copy and build upon published articles even for commercial purposes, as long as the author and publisher are properly credited, which ensures maximum dissemination and a wider impact of our publications. However, the dissemination and distribution of physical copies of this book as a whole is restricted to MDPI, Basel, Switzerland. 


\section{Table of Contents}

List of Contributors V

About the Guest Editors. VIII

Preface IX

Nourdine Zibouche, Mahdi Ghorbani-Asl, Thomas Heine and Agnieszka Kuc

Electromechanical Properties of Small Transition-Metal Dichalcogenide Nanotubes

Reprinted from: Inorganics 2014, 2(2), 155-167

http://www.mdpi.com/2304-6740/2/2/155 1

Volker Brüser, Ronit Popovitz-Biro, Ana Albu-Yaron, Tommy Lorenz, Gotthard Seifert, Reshef Tenne and Alla Zak

Single- to Triple-Wall $\mathrm{WS}_{2}$ Nanotubes Obtained by High-Power Plasma Ablation of $\mathrm{WS}_{2}$ Multiwall Nanotubes

Reprinted from: Inorganics 2014, 2(2), 177-190

http://www.mdpi.com/2304-6740/2/2/177

Claudia C. Luhrs, Michael Moberg, Ashley Maxson, Luke Brewer and Sarath Menon IF-WS $_{2}$ Nanostructured Carbon Hybrids Generation and Their Characterization Reprinted from: Inorganics 2014, 2(2), 211-232 http://www.mdpi.com/2304-6740/2/2/211 .28

Matthew R. Farrow, John Buckeridge, C. Richard A. Catlow, Andrew J. Logsdail, David O. Scanlon, Alexey A. Sokol and Scott M. Woodley

From Stable ZnO and GaN Clusters to Novel Double Bubbles and Frameworks Reprinted from: Inorganics 2014, 2(2), 248-263 http://www.mdpi.com/2304-6740/2/2/248 .50

\section{Mohammed Naffakh and Ana M. Díez-Pascual}

Thermoplastic Polymer Nanocomposites Based on Inorganic Fullerene-like Nanoparticles and Inorganic Nanotubes

Reprinted from: Inorganics 2014, 2(2), 291-312

http://www.mdpi.com/2304-6740/2/2/291 
Fang Xu, Nannan Wang, Hong Chang, Yongde Xia and Yanqiu Zhu

Continuous Production of IF-WS $\mathrm{W}_{2}$ Nanoparticles by a Rotary Process

Reprinted from: Inorganics 2014, 2(2), 313-333

http://www.mdpi.com/2304-6740/2/2/313

.87

\section{Albert Rimola and Mariona Sodupe}

Gas-Phase and Microsolvated Glycine Interacting with Boron Nitride Nanotubes. A B3LYP-

D2* Periodic Study

Reprinted from: Inorganics 2014, 2(2), 334-350

http://www.mdpi.com/2304-6740/2/2/334

Olga Brontvein, Reshef Tenne and Andrey Enyashin

The Role of Lead $(\mathrm{Pb})$ in the High Temperature Formation of $\mathrm{MoS}_{2}$ Nanotubes Reprinted from: Inorganics 2014, 2(2), 363-376

http://www.mdpi.com/2304-6740/2/2/363 130

Jamie Cook, Steven Rhyans, Lou Roncase, Garth Hobson and Claudia C. Luhrs

Microstructural Study of IF-WS 2 Failure Modes

Reprinted from: Inorganics 2014, 2(3), 377-395

http://www.mdpi.com/2304-6740/2/3/377

Georges Moussa, Chrystelle Salameh, Alina Bruma, Sylvie Malo, Umit B. Demirci, Samuel Bernard and Philippe Miele

Nanostructured Boron Nitride: From Molecular Design to Hydrogen Storage Application

Reprinted from: Inorganics 2014, 2(3), 396-409

http://www.mdpi.com/2304-6740/2/3/396

Daniel Raichman, David Strawser and Jean-Paul Lellouche

Design of Experiments: Optimizing the Polycarboxylation/Functionalization of Tungsten

Disulfide Nanotubes

Reprinted from: Inorganics 2014, 2(3), 455-467

http://www.mdpi.com/2304-6740/2/3/455 176

Nourdine Zibouche, Agnieszka Kuc, Pere Miró and Thomas Heine

Noble-Metal Chalcogenide Nanotubes

Reprinted from: Inorganics 2014, 2(4), 556-564

http://www.mdpi.com/2304-6740/2/4/556 190 


\section{List of Contributors}

Ana Albu-Yaron: Department of Materials and Interfaces, Weizmann Institute of Science, P.O. Box 26, Rehovot 76100, Israel.

Samuel Bernard: IEM (Institut Europeen des Membranes), UMR 5635 (CNRS-ENSCMUM2), Universite Montpellier 2, Place E. Bataillon, F-34095 Montpellier, France.

Luke Brewer: Mechanical and Aerospace Engineering Department. Naval Postgraduate School 700 Dyer Rd. Watkins Hall. Monterey, CA 9394, USA.

Olga Brontvein: Department of Materials and Interfaces, Weizmann Institute of Science, Rehovot 7610001, Israel.

Alina Bruma: Laboratoire CRISMAT, UMR 6508 CNRS/ENSICAEN/UCBN, 6 boulevard du Maréchal Juin, 14050 Caen, France.

Volker Brüser: Leibnitz Institute for Plasma Science and Technology (INP), Felix-Hausdorff-Straße 2, 17489 Greifswald, Germany.

John Buckeridge: Department of Chemistry, Kathleen Lonsdale Materials Chemistry, University College London, 20 Gordon Street, London WC1H 0AJ, UK.

C. Richard A. Catlow: Department of Chemistry, Kathleen Lonsdale Materials Chemistry, University College London, 20 Gordon Street, London WC1H 0AJ, UK.

Hong Chang: College of Engineering, Mathematics and Physical Sciences, University of Exeter, Exeter EX4 4QF, UK.

Jamie Cook: Mechanical and Aerospace Engineering Department, Naval Postgraduate School, 700 Dryer Rd., Watkins Hall Rm. 305, Monterey, CA 93943, USA.

Umit B. Demirci: IEM (Institut Europeen des Membranes), UMR 5635 (CNRS-ENSCMUM2), Universite Montpellier 2, Place E. Bataillon, F-34095 Montpellier, France.

Ana M. Díez-Pascual: Instituto de Ciencia y Tecnología de Polímeros (ICTP-CSIC), Juan de la Cierva 3, 28006 Madrid, Spain.

Andrey Enyashin: Institute of Solid State Chemistry RAS Pervomayskaya Str., 91, 620990 Ekaterinburg, Russia.

Matthew R. Farrow: Department of Chemistry, Kathleen Lonsdale Materials Chemistry, University College London, 20 Gordon Street, London WC1H 0AJ, UK.

Mahdi Ghorbani-Asl: School of Engineering and Science, Jacobs University Bremen, Campus Ring 1, 28759 Bremen, Germany.

Thomas Heine: School of Engineering and Science, Jacobs University Bremen, Campus Ring 1, 28759 Bremen, Germany.

Garth Hobson: Mechanical and Aerospace Engineering Department, Naval Postgraduate School, 700 Dryer Rd., Watkins Hall Rm. 305, Monterey, CA 93943, USA.

Agnieszka Kuc: School of Engineering and Science, Jacobs University Bremen, Campus Ring 1, 28759 Bremen, Germany.

Jean-Paul Lellouche: Department of Chemistry, Nanomaterials Research Center, Institute of Nanotechnology \& Advanced Materials, Bar-Ilan University, Ramat-Gan 52900, Israel. 
Andrew J. Logsdail: Department of Chemistry, Kathleen Lonsdale Materials Chemistry, University College London, 20 Gordon Street, London WC1H 0AJ, UK.

Tommy Lorenz: Physikalische Chemie, Technische Universität Dresden, Bergstrasse, 66b, 01062 Dresden, Germany.

Claudia C. Luhrs: Mechanical and Aerospace Engineering Department, Naval Postgraduate School, 700 Dryer Rd., Watkins Hall Rm. 305, Monterey, CA 93943, USA.

Sylvie Malo: Laboratoire CRISMAT, UMR 6508 CNRS/ENSICAEN/UCBN, 6 boulevard du Maréchal Juin, 14050 Caen, France.

Ashley Maxson: Mechanical and Aerospace Engineering Department. Naval Postgraduate School 700 Dyer Rd. Watkins Hall. Monterey, CA 9394, USA.

Sarath Menon: Mechanical and Aerospace Engineering Department. Naval Postgraduate School 700 Dyer Rd. Watkins Hall. Monterey, CA 9394, USA.

Philippe Miele: IEM (Institut Europeen des Membranes), UMR 5635 (CNRS-ENSCMUM2), Universite Montpellier 2, Place E. Bataillon, F-34095 Montpellier, France.

Pere Miró: School of Engineering and Science, Jacobs University Bremen, Campus Ring 1, 28759 Bremen, Germany.

Michael Moberg: Mechanical and Aerospace Engineering Department. Naval Postgraduate School 700 Dyer Rd. Watkins Hall. Monterey, CA 9394, USA.

Georges Moussa: IEM (Institut Europeen des Membranes), UMR 5635 (CNRS-ENSCMUM2), Universite Montpellier 2, Place E. Bataillon, F-34095 Montpellier, France.

Mohammed Naffakh: Departamento de Ingeniería y Ciencia de los Materiales, Escuela Técnica Superior de Ingenieros Industriales, Universidad Politécnica de Madrid, José Gutiérrez Abascal 2, 28006 Madrid, Spain.

Ronit Popovitz-Biro: Department of Chemical Research Support, Weizmann Institute of Science, P.O. Box 26, Rehovot 76100, Israel.

Daniel Raichman: Department of Chemistry, Nanomaterials Research Center, Institute of Nanotechnology \& Advanced Materials, Bar-Ilan University, Ramat-Gan 52900, Israel.

Steven Rhyans: Mechanical and Aerospace Engineering Department, Naval Postgraduate School, 700 Dryer Rd., Watkins Hall Rm. 305, Monterey, CA 93943, USA; Hartnell College, Salinas, CA 93901, USA.

Albert Rimola: Departament de Química, Universitat Autònoma de Barcelona, 08193 Bellaterra, Catalonia, Spain.

Lou Roncase: Navy's Weapons Survivability Laboratory (WSL), Naval Air Warfare Center (NAWC), China Lake, CA 93555, USA.

Chrystelle Salameh: IEM (Institut Europeen des Membranes), UMR 5635 (CNRS-ENSCMUM2), Universite Montpellier 2, Place E. Bataillon, F-34095 Montpellier, France.

David O. Scanlon: Department of Chemistry, Kathleen Lonsdale Materials Chemistry, University College London, 20 Gordon Street, London WC1H 0AJ, UK; Diamond Light Source Ltd., Diamond House, Harwell Science and Innovation Campus, Didcot, Oxfordshire OX11 0DE, UK.

Gotthard Seifert Seifert: Physikalische Chemie, Technische Universität Dresden, Bergstrasse, 66b, 01062 Dresden, Germany. 
Mariona Sodupe: Departament de Química, Universitat Autònoma de Barcelona, 08193 Bellaterra, Catalonia, Spain.

Alexey A. Sokol: Department of Chemistry, Kathleen Lonsdale Materials Chemistry, University College London, 20 Gordon Street, London WC1H 0AJ, UK.

David Strawser: Department of Chemistry, Nanomaterials Research Center, Institute of Nanotechnology \& Advanced Materials, Bar-Ilan University, Ramat-Gan 52900, Israel.

Reshef Tenne: Department of Materials and Interfaces, Weizmann Institute of Science, P.O. Box 26, Rehovot 76100, Israel.

Nannan Wang: College of Engineering, Mathematics and Physical Sciences, University of Exeter, Exeter EX4 4QF, UK.

Scott M. Woodley: Department of Chemistry, Kathleen Lonsdale Materials Chemistry, University College London, 20 Gordon Street, London WC1H 0AJ, UK.

Yongde Xia: College of Engineering, Mathematics and Physical Sciences, University of Exeter, Exeter EX4 4QF, UK.

Fang Xu: College of Engineering, Mathematics and Physical Sciences, University of Exeter, Exeter EX4 4QF, UK.

Alla Zak: Faculty of Science, Holon Institute of Technology, P.O. Box 305, Holon 58102, Israel.

Yanqiu Zhu: College of Engineering, Mathematics and Physical Sciences, University of Exeter, Exeter EX4 4QF, UK.

Nourdine Zibouche: School of Engineering and Science, Jacobs University Bremen, Campus Ring 1, 28759 Bremen, Germany. 


\section{About the Guest Editors}

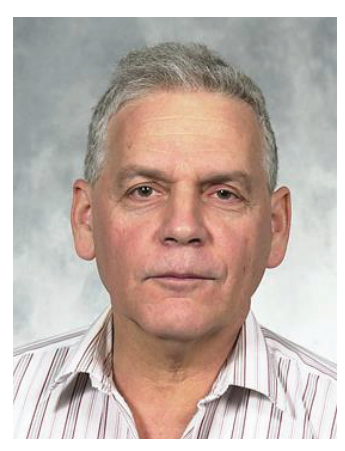

Reshef Tenne earned his Ph.D. in 1976 in the Hebrew University of Jerusalem. He joined the Weizmann Institute in 1979, where he was promoted to a professor in 1995. He headed the Department of Materials and Interfaces and was the director of the G. Schmidt Minerva Center for Supramolecular Chemistry (2000-2007) and the Helen and Martin Kimmel Center for Nanoscale Science (2003-2014).

Prof. Tenne is recognized for the discovery of the inorganic fullerene-like (IF) nanostructures and inorganic nanotubes (INT) of WS2 and MoS2 (in 1992); for their detailed study and for their commercialization as superior solid lubricants. He holds the Drake Family Chair in Nanotechnology (2004-); received the Materials Research Society Medal (2005); The Kolthoff Prize in Chemistry of the Technion, Israel (2005); The Israel Vacuum Society Excellence in Science Prize (2006); The Landau Prize in Nanotechnology (2006); was nominated as MRS Fellow in 2008; receiving the Israel Chemical Society Prize (2008) and the European Research Society (ERC) Advanced Research Grant (2008). In 2011, he was elected to the Israel Academy of Sciences and Academia Europaea and became a Fellow of the Royal Society of Chemistry. He was chosen to deliver the CNR Award Lecture (Indian Chemical Res. Soc.) in 2012 and received the ChinaNano plenary lecture prize (2011). His paper in Nanomaterials and Energy Journal received the best paper award of the year (in 2014) from the Institute of Civil Engineering (UK). Prof. Tenne is recipient of the Rothschild Prize in Physics and Chemistry (2015).

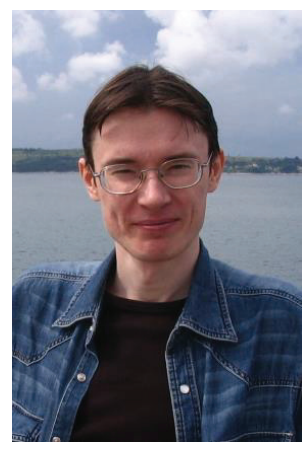

Andrey N. Enyashin received his Ph.D. in chemistry at Ural Technical State University (Yekaterinburg) in 2005. After a postdoc in the theoretical chemistry group of Prof. Gotthard Seifert at the Dresden University of Technology, Germany, he was appointed senior researcher at the Institute of Solid State Chemistry of UB RAS (Russian Federation). He was also a guest scientist at the Donostia International Physics Center, Spain. He is a recipient of the Samsung Electro-Mechanics Medal (2007). Currently, his field of interest is computational materials science of inorganic and carbon nanostructures. 


\section{Preface}

\section{Inorganic Fullerene-Like Nanoparticles and Inorganic Nanotubes}

Fullerene-like nanoparticles (inorganic fullerenes; IF) and nanotubes of inorganic layered compounds (inorganic nanotubes; INT) combine low dimensionality and nanosize, enhancing the performance of corresponding bulk counterparts in their already known applications, as well as opening new fields of their own. This issue gathers articles from the diverse area of materials science and is devoted to fullerene-like nanoparticles and nanotubes of layered sulfides and boron nitride, and collating the most current results obtained at the interface between fundamental research and engineering.

Arising from a fortuitous lab discovery, the commercial production of inorganic hollow nanoparticles was focused on molybdenum and tungsten disulfides. Their superior solid lubrication effects have engendered intense industrial scale-up and commercialization, with sales of thousands of tons of formulated lubricants per year. Yet, the search and evaluation of more cost-effective and environmentally friendly manufacturing technologies continues. The paper by $\mathrm{Xu}$ et al. published recently recent "Continuous Production of IF-WS $\mathrm{W}_{2}$ Nanoparticles by a Rotary Process" describes an attempt for further rationalization and scale-up of the manufacturing of $\mathrm{WS}_{2}$ nanoparticles after gas-solid reductive sulfurization of $\mathrm{WO}_{3}$ nanoparticles in a rotary furnace. This systematic study included the investigation of many reaction parameters, such as precursor type, reaction temperature and time, and the reducing atmosphere. This new technique could, in the future, become a successful alternative for increasing the yield of IF production compared to the current fluidized-bed reactor.

The fullerene-like morphology of $\mathrm{MoS}_{2}$ and $\mathrm{WS}_{2}$ considerably improves the tribological properties of these compounds, pushing ahead the large-scale use of layered sulfides in machinery, aerospace and, in the future, also in medical industries as dry and oil-based lubricants as well as wear-resistant surface coatings. Such applications require deep understanding of the factors determining the mechanical and structural stability of inorganic nanoparticles under extreme conditions of high pressure or intense irradiation. The study of Cook et al. "Microstructural Study of IF-WS ${ }_{2}$ Failure Modes" explores the failure mechanisms found in $\mathrm{WS}_{2}$ Ifs, treated with diverse pressure loading methods. The authors uncover at least two distinct fracture modes, i.e., the collapse of quasi-spherical morphology into agglomerated plate-like sheets and the delamination and exfoliation of the IF-WS nanoparticles. The latter process is accomplished by inductively-coupled radio-frequency plasma irradiation of multiwall $\mathrm{WS}_{2}$ nanotubes, which is discussed in another paper "Single-to Triple-Wall $\mathrm{WS}_{2}$ Nanotubes Obtained by High-Power Plasma Ablation of $\mathrm{WS}_{2}$ Multiwall Nanotubes" in this issue. The authors were able to control the process of layer-by-layer "undressing" of multilayered INTs and, in this manner, fabricate $\mathrm{WS}_{2}$ nanotubes with ultrathin (one to three layers) walls. 
The significant stability of hollow sulfide nanoparticles (IF/INT) under shock-wave propagation or irradiation suggests their potential use as fillers for impact resilient polymer or ceramic composites. Apparently, such applications of $\mathrm{WS}_{2}$ or $\mathrm{MoS}_{2}$ fullerene-like particles or nanotubes may yield polymer composites with a high degree of crystallinity and, consequently, with improved thermoplastic and mechanical properties, as demonstrated by Naffakh et al. in the paper "Thermoplastic Polymer Nanocomposites Based on Inorganic Fullerene-Like Nanoparticles and Inorganic Nanotubes". However, in many cases, the adhesion between a nanoparticle and the polymer matrix is limited to the weak van der Waals interaction and could be enhanced by means of covalent bonding at the nanoparticle-polymer interface. Surface functionalization of IFs or INTs, as reported by Raichmann et al. in "Design of Experiments: Optimizing the Polycarboxylation/Functionalization of Tungsten Disulfide Nanotubes", could provide stronger adhesion of nanoparticles with the polymer matrix. The emphasis in this direction was given to the non-trivial functionalization of the hydrophobic $\mathrm{WS}_{2}$ nanotubes by hydrophilic carboxyl groups, which could further stimulate the fabrication of hydrophilic polymer composites or ceramics.

Substantial progress in safe production and pioneering use of IFs and INTs has been made possible due to the comprehensive experimental research of their formation conditions, chemical activity, mechanical and electronic characteristics. However, novel and modified nanoparticles of sulfides and other compounds, such as boron nitride, can provide a much larger diversity of new materials in catalysis, electronics and electrochemistry, and their detailed characterization is still required. In the paper "Nanostructured Boron Nitride: from Molecular Design to Hydrogen Storage Application", high-temperature spray-pyrolysis synthesis of hollow-core BN nanoparticles was demonstrated. The synthesized nanoparticles were carefully characterized and studied as a host for hydrogen storage applications.

Computational materials science can be a valuable tool for a preliminary study of this kind of nanoparticles and this issue contains examples of theoretical papers describing investigations of this type. For example, the paper "Gas-Phase and Microsolvated Glycine Interacting with Boron Nitride Nanotubes: A B3LYP-D2* Periodic Study" examines the adsorption of the amino-acid glycine on the surface of zig-zag BN nanotubes. Pure and solvated glycine moieties have been investigated. In several cases, chemisorption was found to be important, while in others $\pi-\pi$ stacking, or through water molecules, was found to be more relevant. In another study, nanotubes of noble-metal dichalcogenides were designed and described as stable semiconductors in theoretical work by Zibouche et al.: "Noble-Metal Chalcogenide Nanotubes". It can guide experimental groups in researching fullerene-like nanoparticles and nanotubes of other compounds. In another theoretical paper: "From Stable ZnO and GaN Clusters to Novel Double Bubbles and Frameworks", bottom-up construction of hollow clusters ("bubbles") of high-symmetry were systematically studied.

The subjects of the presented papers cover a wide range of challenges in the area of inorganic fullerene-like nanoparticles and nanotubes. However, it can include only a few comprehensive experimental and theoretical efforts, stepwise evaluating the rationalization of the synthesis, and elucidation of the stability, mechanical, electronic and adhesive properties of these nanostructures. We believe that this thematic issue can be helpful, not only for an 
advanced researcher to grasp the latest developments in this field, but also to permit a beginner to gain a deeper insight into the field of inorganic fullerene-like nanoparticles and nanotubes.

Reshef Tenne and Andrey N. Enyashin

Guest Editors 



\title{
Electromechanical Properties of Small Transition-Metal Dichalcogenide Nanotubes
}

\section{Nourdine Zibouche, Mahdi Ghorbani-Asl, Thomas Heine and Agnieszka Kuc}

\begin{abstract}
Transition-metal dichalcogenide nanotubes (TMC-NTs) are investigated for their electromechanical properties under applied tensile strain using density functional-based methods. For small elongations, linear strain-stress relations according to Hooke's law have been obtained, while for larger strains, plastic behavior is observed. Similar to their 2D counterparts, TMC-NTs show nearly a linear change of band gaps with applied strain. This change is, however, nearly diameter-independent in case of armchair forms. The semiconductor-metal transition occurs for much larger deformations compared to the layered tube equivalents. This transition is faster for heavier chalcogen elements, due to their smaller intrinsic band gaps. Unlike in the 2D forms, the top of valence and the bottom of conduction bands stay unchanged with strain, and the zigzag NTs are direct band gap materials until the semiconductor-metal transition. Meanwhile, the applied strain causes modification in band curvature, affecting the effective masses of electrons and holes. The quantum conductance of TMC-NTs starts to occur close to the Fermi level when tensile strain is applied.
\end{abstract}

Reprinted from Inorganics. Cite as: Zibouche, N.; Ghorbani-Asl, M.; Heine, T.; Kuc, A. Electromechanical Properties of Small Transition-Metal Dichalcogenide Nanotubes. Inorganics

2014, 2, 155-167.

\section{Introduction}

In the past few years, transition-metal dichalcogenides (TMCs) have become a class of materials most widely investigated in the fields of physics, materials science or nanotechnology. Especially, two-dimensional (2D) layered forms of TMCs are of great interest, as they can be easily manufactured to monolayers using chemical or mechanical exfoliation and chemical deposition techniques [1-3]. They possess desirable intrinsic band gaps ranging from about 1.0 to $2.0 \mathrm{eV}$, and they were utilized in nanoelectronic applications to produce field-effect transistors, logical circuits, amplifiers and photodetectors [4-7]. The electronic properties of 2D TMCs can be tuned by various means, including quantum confinement [8-11], mechanical deformations [12-14], electric fields [15,16] or local defects [17-19].

Similar to carbon, tubular and fullerene-like nanostructures can be formed from other inorganic materials, including sulfo-carbides [20], boron-carbon-nitrides [21] or TMCs [22,23]. Though less than their carbon counterparts, in particular $\mathrm{MoS}_{2}$ and $\mathrm{WS}_{2}$ nano-onions and nanotubes have been investigated both theoretically and experimentally [24-30]. TMC nanotubes (TMCs-NTs) behave as exceptional lubricants [31,32], and it has been shown that when the $\mathrm{MoS}_{2}$ NTs or nano-onions are added to base grease, the friction coefficient remains low, even at very high loads [33]. The mechanical properties of TMC-NTs have been investigated experimentally, where tubes were subject 
to tensile strain using atomic force microscopy [34-37]. Elastic deformations were predicted from linear strain-stress relation up to the fracture point (at $13 \mathrm{GPa}$ and $12 \%$ strain for $\mathrm{WS}_{2}$ TMC-NTs), and fracture was directly related to the formation of local defects [35]. The mechanical properties of $\mathrm{WS}_{2}$ NTs under axial tension and compression [36] shows that they are ultra-strong and elastic, which distinguishes them from other known materials. Quantum-mechanical simulations showed that under squeezing, $\mathrm{MoS}_{2}$ NTs start to form platelets, partially attached to the grips, which provide good lubrication at the position of closest contact. This is interpreted as 'nano-coating'.

Single-walled TMC-NTs have interesting electronic properties that depend on their diameter and chirality. Zigzag $(\mathrm{n}, 0)$ NTs are direct band gap semiconductors, resembling $1 H$ TMC forms, while armchair (n,n) NTs are indirect band gap materials, similar to the $2 H$ TMC structures [24,29]. Zigzag tubes are, therefore, suggested for luminescent devices, an application that would not be possible for carbon NTs. With increasing tube diameter, the band gaps increase and eventually approach the single-layer limit.

Doping inorganic semiconducting NTs may lead to new optoelectronic nanomaterials. Ivanovskaya et al. [38] have investigated the effect of Mo to $\mathrm{Nb}$ doping on the electronic structure of $\mathrm{MoS}_{2}$ NTs using the density functional based tight-binding (DFTB) method. It has been found that composite $\mathrm{Mo}_{1-x} \mathrm{Nb}_{x} \mathrm{~S}_{2} \mathrm{NTs}$ are more stable than the corresponding mixture of pure tubes. This effect was even stronger for larger tube diameters. The authors reported that all doped NTs were metallic, independent of their chirality, diameters or the substitutional patterns. The density of states close to the Fermi level of Nb-substituted $\mathrm{MoS}_{2}$ NTs can be tuned in a wide range by the degree of doping.

Electromechanical properties have been widely investigated theoretically for TMC monolayers $[14,39,40]$, but they remain to be explored for the associated tubular structures. Because of their excellent lubricating properties, the application of tensile stress on 2D TMC systems is rather difficult in experiments. The experimental setup for direct tensile tests of TMC-NTs is, however, state-of-the-art [35,37]. We have recently shown that the electronic properties of large-diameter TMC-NTs can be tuned by an external tensile strain for nanoelectromechanical applications [41] and that Raman spectroscopy is an ideal tool to monitor the strain of the individual tubes due to a linear correlation between the Raman shift and the strain. These results hold, however, for large diameter nanotubes. For small diameter tubes, finite size effects are expected.

In this work, we have investigated the electromechanical properties of small diameter TMC nanotubes by applying axial tensile strain. Stress-strain relations, the electronic structure and quantum conductance response to the mechanical deformations were compared between Mo- and W-based NTs with different chalcogen atoms. The results were compared to the available experimental and theoretical works. Our calculations show that up to $2 \%-5 \%$ elongations, the stress-strain relations scale linearly, and we obtained Young's moduli of about $200 \mathrm{GPa}$ for armchair and zigzag tubes, with the notable exception of much softer $\mathrm{WTe}_{2}$. The shape of the band structures is strongly affected, the conduction bands have a loose dispersion for zigzag tubes, while the dispersion deepens for armchair materials. We find nearly a linear decrease in the band gap for all types of nanotubes, and eventually, the semiconductor-metal transition occurs. This is, however, observed at 
larger elongations than for the corresponding layered forms. Tensile strain enhances conductance closer to the Fermi level.

\section{Computational Details}

We have investigated the $(\mathrm{n}, \mathrm{n})$ armchair and the $(\mathrm{n}, 0)$ zigzag $\mathrm{TX}_{2}$ nanotubes $(\mathrm{T}=\mathrm{Mo}$, W; $\mathrm{X}=\mathrm{S}, \mathrm{Se}, \mathrm{Te}$ ) with $n=21$ and 24 (see Figure 1). All structures were fully optimized (atomic positions and lattice constants) employing helical boundary conditions as implemented in the Crystal09 software package [42].

Figure 1. Front and sided views of zigzag and armchair $\mathrm{TX}_{2} \mathrm{NT}$ structures at equilibrium and under tensile strain $(\varepsilon)$.

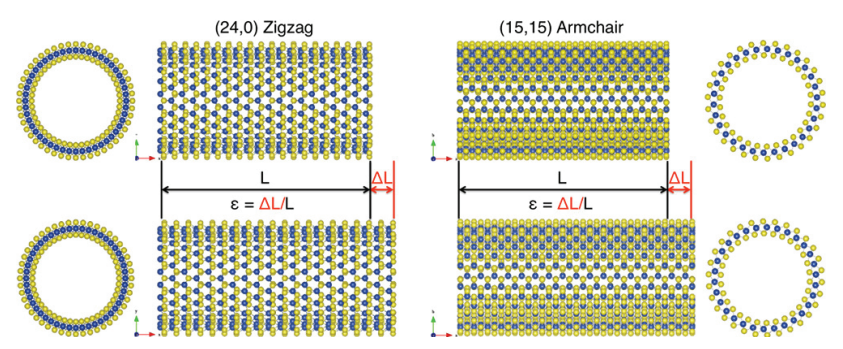

For the strained structures, only the atomic coordinates have been re-optimized, while the unit cell parameter along the tube axis was kept fixed, as reported in our previous works [14,41]. The tensile strain is defined as $\varepsilon=\left(L-L_{0}\right) / L_{0}$, where $L_{0}$ and $L$ are equilibrium and strained lattice values, respectively ( $c f$. Figure 1). The elastic properties of the tubes under tensile stress were calculated as force, F, acting on the area, A. The area can be calculated as follows:

$$
A=2 \pi R_{0} \delta
$$

where $R_{0}$ is the tube radius, defined as the distance between the center of the tube and the metal atom, and $\delta$ is the thickness of tube wall, taken as the interlayer distance of the bulk material. The Young's modulus, $Y$, is obtained from the second derivative of the total energy with respect to the applied strain at the equilibrium volume, $V_{0}$ :

$$
Y=\frac{1}{V_{0}} \frac{\partial^{2} E}{\partial \varepsilon^{2}}
$$

where $V_{0}=A L_{0}$.

Structural and electromechanical properties have been calculated using density functional theory (DFT) in the representation by Perdew, Burke and Ernzerhof (PBE) [43], a method that was validated for the TMC systems earlier [11,29,41]. The all-electron $86-311 \mathrm{G}^{*}$ basis was chosen for sulfur atoms, while for the heavier elements, the effective core potential (ECP) approach with large cores was employed, accounting for scalar relativistic effects $[44,45]$. The shrinking factor was set to 
eight, resulting in $5 k$ points in the irreducible Brillouin zone according to the Monkhorst-Pack sampling [46]. Band structures were calculated along the high symmetry points using the $\Gamma-X$ path.

The coherent electronic transport calculations were carried out using the density functional based tight-binding (DFTB) [47-49] method in conjunction with the non-equilibrium Green's function technique $[50,51]$ and the Landauer-Büttiker approach. The present approach was already validated and described in detail in our previous works on various TMC materials [14,41,52].

\section{Results and Discussion}

We have calculated the electromechanical properties of $\mathrm{TX}_{2}$ nanotubes by applying tensile strain $(\varepsilon)$ to the tubes along their axis. Tensile strain causes changes in the geometry and results in the elongation of the T-X bonds (see Figure 2). These bond lengths increase nearly linearly with $\varepsilon$ and are more sever for zigzag NTs. This trend is similar to the corresponding mechanical deformations in the 2D TMC structures [14,41]. In nanotubes, one needs to distinguish between T-X bond lengths in the outer and inner walls, the latter being slightly shorter. While for armchair NTs, outer and inner bonds change in the same way, this is not the case for zigzag NTs. At larger $\varepsilon$, the inner bonds undergo stronger elongations, eventually approaching the same values as for the outer bonds. We obtain elongation of $0.8-1.0 \mathrm{pm}$ and $0.5-0.6 \mathrm{pm}$ per $1 \%$ of strain for the zigzag and armchair NTs, respectively. Stronger elongations of bonds in the zigzag NTs can be understood, such that along the tube, where the tensile strain is applied, there are many bonds oriented exactly parallel to the axis, while this is not the case in armchair tubes. These bonds can be easily stretched, resulting also in a reduction of the $\mathrm{X}-\mathrm{T}-\mathrm{X}$ angles.

Once the tubes are subject to $\varepsilon$, also the tube diameters change, namely they have to shrink to compensate for the elongations along the tube axis. On average, the tube diameters shrink by $0.6 \AA$ and $0.2 \AA$ per $1 \%$ of strain for zigzag and armchair NTs, respectively.

The stress-strain relations for all the studied tubes are shown in Figure 3. If the curves are fitted to the harmonic approximation for small deformations according to Hooke's law, the stress-strain plots are linear, and the plastic deformations for larger strain values could not be observed. From this fitting, however, we have obtained the Young's moduli for all the tubes (see Table 1). Our results are in agreement with the available experimental and theoretical values. For example, the experimental Young's modulus of multi-wall $\mathrm{WS}_{2}$ NTs is found to be $152 \mathrm{GPa}$ [36], $171 \mathrm{GPa}$ [34] and $223 \mathrm{GPa}$ [37]. For single-wall $\mathrm{MoS}_{2}$ NT ropes, the lowest measured Young's modulus was $120 \mathrm{GPa}$ [26], whereas theoretical values estimated from DFTB calculations for $\mathrm{MoS}_{2}$ NTs are 200 $\mathrm{GPa}$ [53] and $230 \mathrm{GPa}$ [30,54]. Moreover, Li et al. [55] have reported 150 and $127 \mathrm{GPa}$ for $(6,6)$ and (10,0) $\mathrm{MoS}_{2}$ NTs, respectively.

If the curves are fitted to a higher order polynomial (here, the fourth order polynomial was chosen), we observe that already, for the $\varepsilon$ of $3 \%-5 \%$ (for sulfides and selenides) or $2 \%-3 \%$ (for tellurides), the curves deviate from linearity. 
Figure 2. The metal-chalcogen bond length $(\mathrm{T}-\mathrm{X})$ change with the applied tensile strain of exemplary $\mathrm{MoS}_{2}$ and $\mathrm{WSe}_{2}$ NTs. Similar linear changes are obtained for other transition-metal dichalcogenide-NTs.

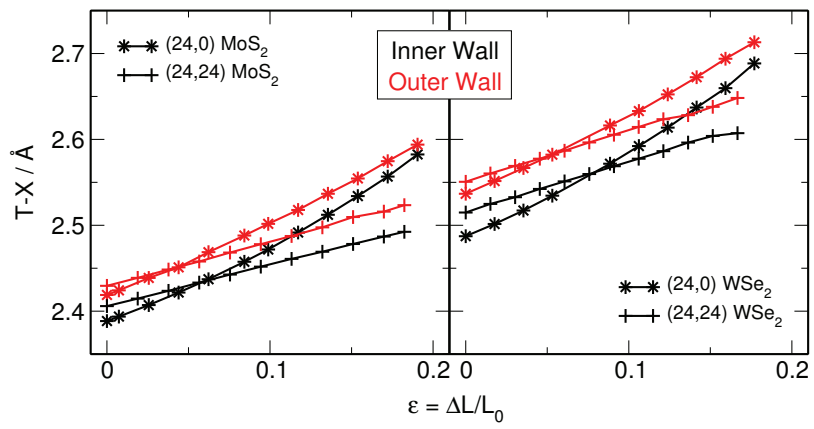

Figure 3. The calculated strain-stress relation of $\mathrm{TX}_{2} \mathrm{NTs}$ under applied tensile strain along the tube axis. Note the different scale on $x$ - and $y$-axes of $\mathrm{TTe}_{2} \mathrm{NTs}_{\text {. }}$

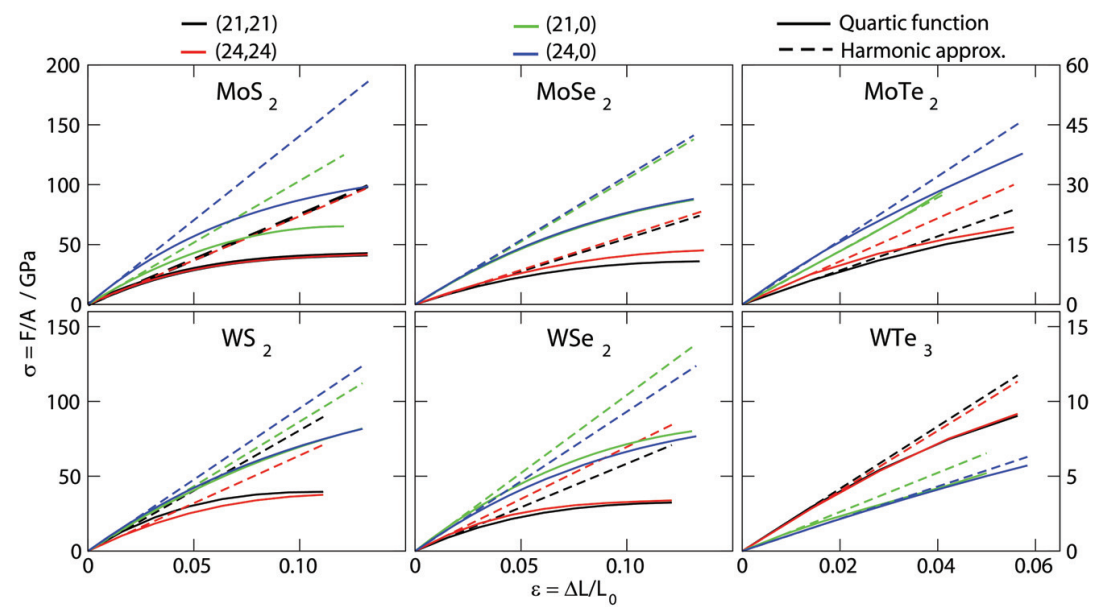

Table 1. The calculated Young's moduli of all the studied NTs. The numbers are obtained from the harmonic approximation following Hooke's law for small values of tensile strain.

\begin{tabular}{ccccc}
\hline \multirow{2}{*}{ System } & \multicolumn{4}{c}{ Chirality } \\
\cline { 2 - 5 } & $\mathbf{( 2 1 , 0 )}$ & $\mathbf{( 2 4 , 0 )}$ & $\mathbf{( 2 1 , 2 1 )}$ & $\mathbf{( 2 4 , 2 4 )}$ \\
\hline $\mathrm{MoS}_{2}$ & 191 & 259 & 235 & 232 \\
$\mathrm{MoSe}_{2}$ & 184 & 188 & 165 & 174 \\
$\mathrm{MoTe}_{2}$ & 110 & 132 & 119 & 150 \\
$\mathrm{WS}_{2}$ & 160 & 177 & 256 & 203 \\
$\mathrm{WSe}_{2}$ & 184 & 165 & 177 & 211 \\
$\mathrm{WTe}_{2}$ & 44 & 36 & 59 & 57 \\
\hline
\end{tabular}


Changes in the geometry of TMC NTs under mechanical deformations also affect the electronic structure of these materials. The band structure responses to the tensile strain are shown in Figures 4 and 5 for zigzag and armchair NTs, respectively. In the equilibrium, zigzag NTs are direct band gap semiconductors at $\Gamma$, while armchair NTs are indirect band gap materials with the valence band maximum (VBM) at $\Gamma$ and the conduction band minimum $(\mathrm{CBM})$ at $2 / 3$ between $\Gamma$ and $X$. This is in close agreement with the DFTB calculations of Seifert et al. [24]. These features are unaffected by $\varepsilon$; however, the CBM of armchair tubes shifts slightly towards the $X$ point.

Figure 4. The calculated band structure response to the applied tensile strain of zigzag $\mathrm{TX}_{2}$ NTs. (a) $\mathrm{MoX}_{2}$ and (b) $\mathrm{WX}_{2}$.
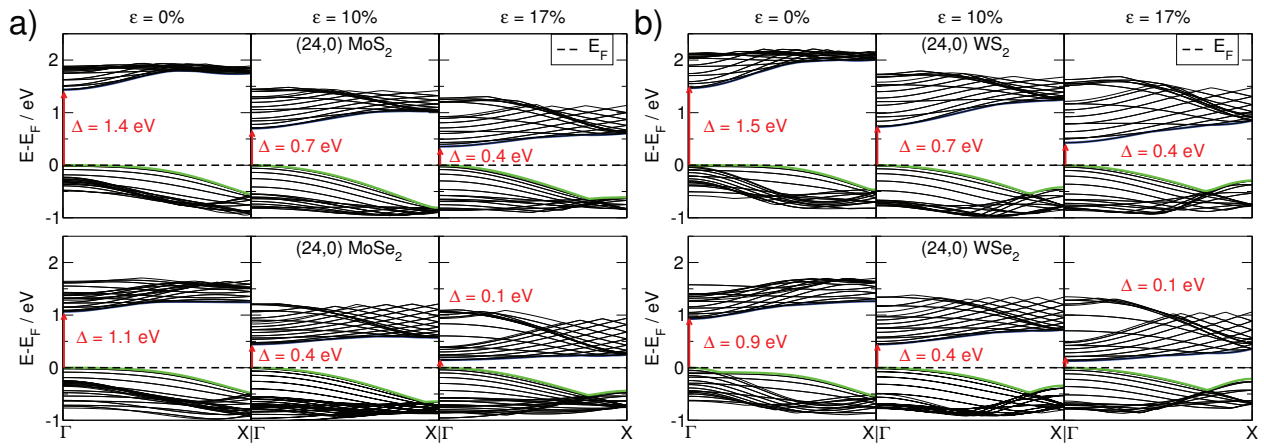

Figure 5. The calculated band structure response to the applied tensile strain of armchair $\mathrm{TX}_{2}$ NTs. (a) $\mathrm{MoX}_{2}$ and (b) $\mathrm{WX}_{2}$.
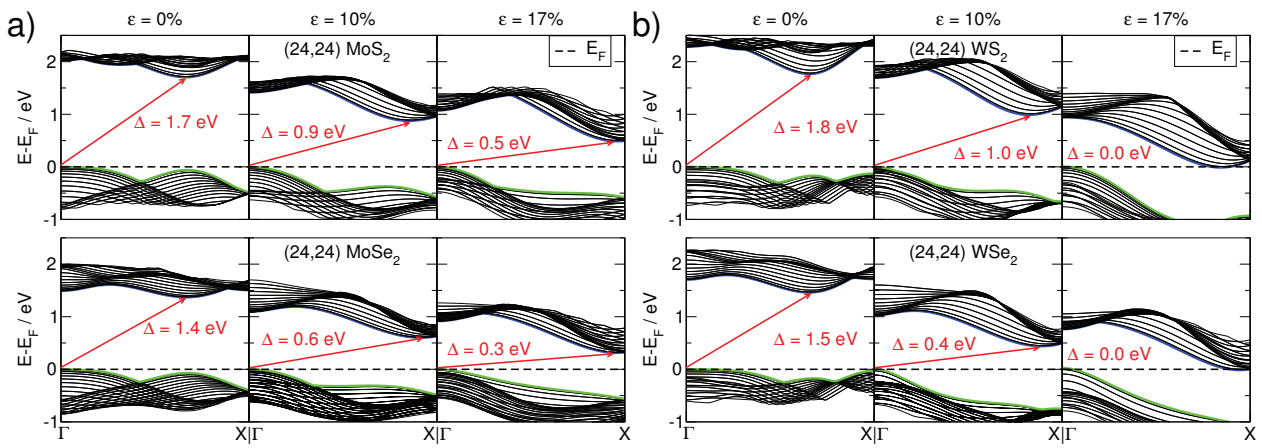

Both the valence and the conduction bands are affected by the mechanical deformations. While in the zigzag NTs, the CBM looses its dispersion with the applied strain, it is opposite in the case of armchair tubes. In the latter, the dispersion deepens, and the CBM position shifts towards the $X$ point. The valence bands get more dispersed along the $\Gamma-X$ paths for larger deformations, and this is chirality-independent.

The band gap evolution with the tensile strain is shown in Figure 6. Nearly linear scaling is found for $\varepsilon$ of $10 \%-12 \%$. The semiconductor-metal transition occurs for elongations much larger than in 
the case of layered 2D forms [14,41], but it is faster for the NTs with heavier chalcogen atoms, as they have a smaller intrinsic band gap. We have noticed that for armchair NTs, there is almost no band gap dependency on the tube diameter for the whole range of $\varepsilon$, as it is in the zigzag forms.

Figure 6. The calculated band gap evolution with the applied tensile strain of zigzag and armchair $\mathrm{TX}_{2}$ NTs.

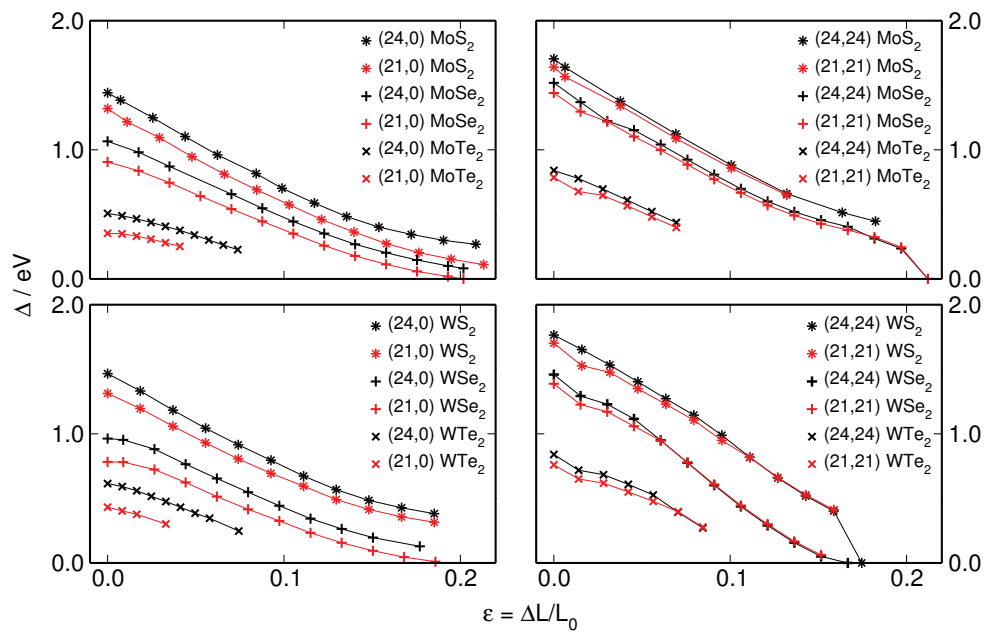

Figure 7 shows the intrinsic quantum conductance $(\mathcal{G})$ calculated along the $\mathrm{TX}_{2} \mathrm{NTs}$ with respect to the applied tensile strain. As the materials are stretched along the tube axis, $\mathcal{G}$ starts to appear closer to the Fermi level, and eventually, the transport channel opens. For all NTs, the conductance below the Fermi level reduces with strain; however, above the $\mathrm{E}_{\mathrm{F}}$, it stays unchanged (it increases) for zigzag (armchair) NTs. Our quantum transport calculations aim to describe the intrinsic conductance of the entire tubes along their principal symmetry axis. Note that the quantum transport calculations are carried out using the DFTB method, which tends to overestimate the electronic band gap.

We have calculated the effective masses of electrons and holes at the CBM and VBM, respectively (see Table 2). The effective masses of holes are reduced with the tensile strain, which is consistent with stronger dispersion in the VBM. The masses of electrons at the equilibriums are similar for zigzag and armchair NTs of the same type. These numbers are larger for heavier chalcogen atoms, similar to the electron effective masses. We expect the effective masses of electrons to increase (decrease) for zigzag (armchair) NTs with $\varepsilon$ as the dispersion of bands decreases (increases). The effective masses are calculated from the harmonic approximation and fitting to the energy point close to the VBM and CBM. This, therefore, strongly depends on the number of $k$-points along the path in the Brillouin zone. We have chosen very fine $k$-point sampling of 150 points between $\Gamma$ and $X$. Thus, we do not observe as clear trends as expected in the effective masses of electrons. For the $(6,6)$ and (10,0) $\mathrm{MoS}_{2}$ NTs, Li et al. [55] have obtained effective masses of electrons and holes of 0.53, $0.51,0.83$ and 1.55 , for armchair and zigzag forms, respectively. 
Figure 7. The electron quantum conductance of $\mathrm{TX}_{2}$ NTs under applied tensile strain along the tube axis.
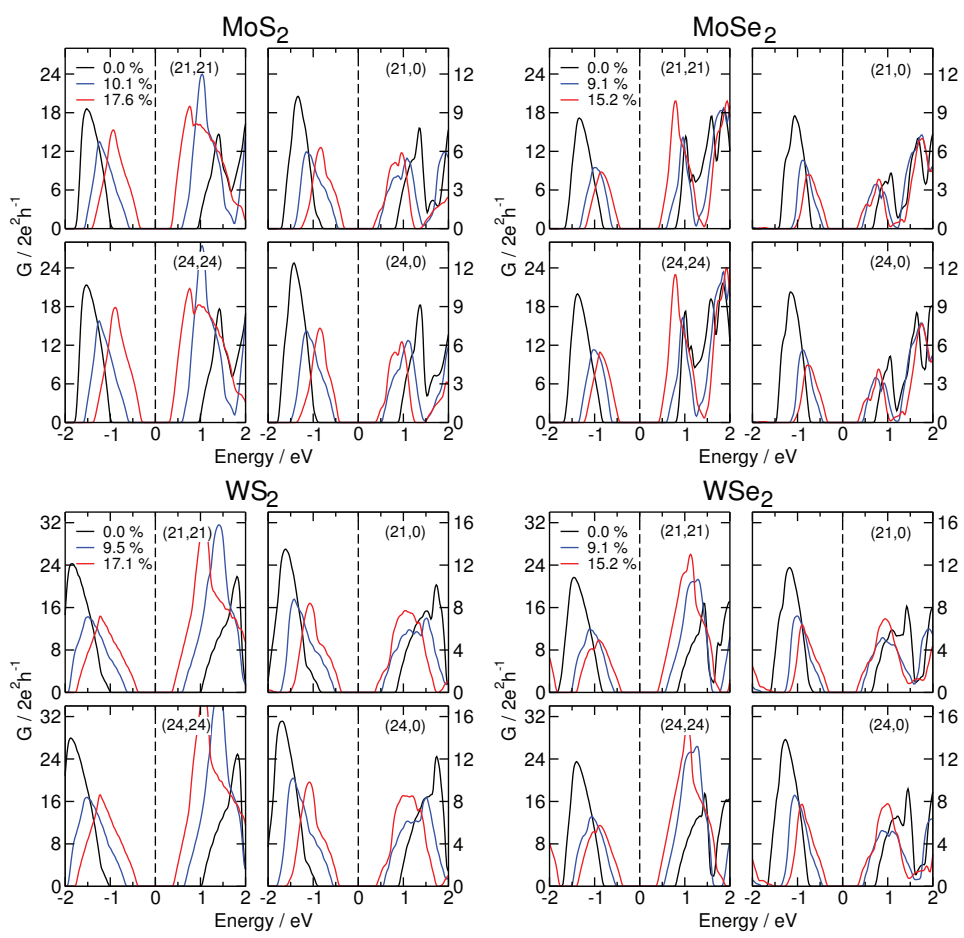

Table 2. The calculated effective electron and hole masses (in $\mathrm{m}_{0}$ units) of $\mathrm{TX}_{2} \mathrm{NTs}$ with respect to the applied tensile strain $(\varepsilon)$. For zigzag NTs, both effective masses are calculated at the $\Gamma$ point; for armchair NTs, effective masses of holes are calculated at $\Gamma$ and of electrons between $\Gamma$ and $X$. Note, for the latter, we do not specify the exact $k$-point as the conduction band minimum (CBM) shifts with $\varepsilon$. The negative values of hole effective masses come from the band curvature at the valence band maximum (VBM).

\begin{tabular}{cccccccc}
\hline \multirow{2}{*}{ System } & \multirow{2}{*}{ Chirality } & \multicolumn{3}{c}{ Electron masses } & \multicolumn{3}{c}{ Hole masses } \\
\cline { 3 - 8 } & & $\mathbf{0 \%}$ & $\mathbf{5 \%}$ & $\mathbf{1 0 \%}$ & $\mathbf{0 \%}$ & $\mathbf{5 \%}$ & $\mathbf{1 0 \%}$ \\
\hline \multirow{2}{*}{$\mathrm{MoS}_{2}$} & $(24,24)$ & 0.621 & 0.631 & 0.707 & -6.209 & -1.684 & -0.974 \\
& $(24,0)$ & 0.655 & 0.621 & 0.660 & -10.03 & -1.990 & -1.033 \\
\hline \multirow{2}{*}{$\mathrm{MoSe}_{2}$} & $(24,24)$ & 0.868 & 0.938 & 0.884 & -15.366 & -2.233 & -1.140 \\
& $(24,0)$ & 1.092 & 0.998 & 0.739 & -0.485 & -3.079 & -3.461 \\
\hline \multirow{2}{*}{$\mathrm{WS}_{2}$} & $(24,24)$ & 0.434 & 0.457 & 0.524 & -11.498 & -1.934 & -0.498 \\
& $(24,0)$ & 0.475 & 0.434 & 0.467 & -12.348 & -3.171 & -1.270 \\
\hline \multirow{2}{*}{$\mathrm{WSe}_{2}$} & $(24,24)$ & 0.621 & 0.715 & 0.659 & -15.147 & -2.285 & -7.922 \\
& $(24,0)$ & 0.585 & 0.714 & 0.916 & -1.362 & -2.846 & -1.396 \\
\hline \multirow{2}{*}{} & & & & & & &
\end{tabular}




\section{Conclusions}

We have investigated the electromechanical properties of inorganic nanotubes of the $\mathrm{TX}_{2}$-type under applied tensile strain. The tubes undergo changes in geometry, namely the $\mathrm{T}-\mathrm{X}$ bond lengths are elongated. More pronounced changes are obtained for the zigzag NTs, in which some of the bonds are oriented parallel to the tube axis, which means along the acting deformation force. The stress-strain relation fitted to the harmonic approximation for small deformations gives Young's moduli of about $200 \mathrm{GPa}$, with the exception of $\mathrm{WTe}_{2}$, which produces notably smaller values around $50 \mathrm{GPa}$.

The electronic properties and the quantum transport are particularly affected by mechanical deformations. Nearly a linear change in the band gap is observed for elongations up to $12 \%$. The semiconductor-metal transition is eventually obtained for all type of tubes; however, it is much faster for heavier chalcogen atoms. Nanotubes require larger tensile strain to become metallic than the corresponding $2 \mathrm{D}$ materials.

The dispersion of the valence and conduction bands changes strongly with the applied strain. Notably, the VBM deepens the dispersion, which results in the lowering of the hole effective masses. The CBM is chirality dependent, and the dispersion is lost (enhanced) for zigzag (armchair) NTs. The transport channels start to open closer to the Fermi level for larger $\varepsilon$.

The electronic properties and the possibility to tune by tensile strain suggest that inorganic NTs, such as TMC materials, could be considered in nanoelectronic applications, for example as switching materials.

\section{Acknowledgments}

This work was supported by the German Research Council (Deutsche Forschungsgemeinschaft, HE 3543/18-1), the European Commission (FP7-PEOPLE-2009-IAPP QUASINANO, GA 251149 and FP7-PEOPLE-2012-ITN MoWSeS, GA 317451).

\section{Author Contributions}

N. Zibouche, M. Ghorbani-Asl, A. Kuc and T. Heine generated, analyzed and discussed the results. T. Heine conceived of this project. All authors contributed in writing this paper.

\section{Conflicts of Interest}

The authors declare no conflict of interest.

\section{References}

1. Coleman, J.N.; Lotya, M.; O’Neill, A.; Bergin, S.D.; King, P.J.; Khan, U.; Young, K.; Gaucher, A.; De, S.; Smith, R.J.; et al. Two-Dimensional Nanosheets Produced by Liquid Exfoliation of Layered Materials. Science 2011, 331, 568-571. 
2. Novoselov, K.S.; Jiang, D.; Schedin, F.; Booth, T.J.; Khotkevich, V.V.; Morozov, S.V.; Geim, A.K. Two-dimensional atomic crystals. Proc. Nat. Acad. Sci. USA 2005, 102, 10451-10453.

3. Lee, Y.H.; Zhang, X.Q.; Zhang, W.; Chang, M.T.; Lin, C.T.; Chang, K.D.; Yu, Y.C.; Wang, J.T.S.W.; Chang, C.S.; Li, L.J.; et al. Synthesis of Large-Area $\mathrm{MoS}_{2}$ Atomic Layers with Chemical Vapor Deposition. Adv. Mater. 2012, 24, 2320-2325.

4. Radisavljevic, B.; Radenovic, A.; Brivio, J.; Giacometti, V.; Kis, A. Single-layer $\mathrm{MoS}_{2}$ transistors. Nat. Nanotechnol. 2011, 6, 147-150.

5. Radisavljevic, B.; Whitwick, M.B.; Kis, A. Integrated Circuits and Logic Operations Based on Single-Layer $\mathrm{MoS}_{2}$. ACS Nano 2011, 5, 9934-9938.

6. Radisavljevic, B.; Whitwick, M.B.; Kis, A. Small-signal amplifier based on single-layer $\mathrm{MoS}_{2}$. Appl. Phys. Lett. 2012, 101, 043103:1-043103:4.

7. Lopez-Sanchez, O.; Lembke, D.; Kayci, M.; Radenovic, A.; Kis, A. Ultrasensitive photodetectors based on monolayer $\mathrm{MoS}_{2}$. Nat. Nanotechnol. 2013, 8, 497-501.

8. Li, T.S.; Galli, G.L. Electronic properties of $\mathrm{MoS}_{2}$ nanoparticles. J. Phys. Chem. C 2007, 111, 16192-16196.

9. Splendiani, A.; Sun, L.; Zhang, Y.B.; Li, T.S.; Kim, J.; Chim, C.Y.; Galli, G.; Wang, F. Emerging Photoluminescence in Monolayer $\mathrm{MoS}_{2}$. Nano Lett. 2010, 10, 1271-1275.

10. Ramakrishna Matte, H.S.S.; Gomathi, A.; Manna, A.K.; Late, D.J.; Datta, R.; Pati, S.K.; Rao, C.N.R. $\mathrm{MoS}_{2}$ and $\mathrm{WS}_{2}$ Analogues of Graphene. Angew. Chem. Int. Ed. 2010, 49, 4059-4062.

11. Kuc, A.; Zibouche, N.; Heine, T. Influence of quantum confinement on the electronic structure of the transition metal sulfide $\mathrm{TS}_{2}$. Phys. Rev. B 2011, 83, 245213:1-245213:4.

12. Bertolazzi, S.; Brivio, J.; Kis, A. Stretching and Breaking of Ultrathin $\mathrm{MoS}_{2}$. ACS Nano 2011, 5, 9703-9709.

13. Castellanos-Gomez, A.; Poot, M.; Steele, G.A.; van der Zant, H.S.J.; Agrait, N.; Rubio-Bollinger, G. Elastic Properties of Freely Suspended $\mathrm{MoS}_{2}$ Nanosheets. Adv. Mater. 2012, 24, 772-775.

14. Ghorbani-Asl, M.; Borini, S.; Kuc, A.; Heine, T. Strain-dependent modulation of conductivity in single layer tranisition metal dichalcogenides. Phys. Rev. B 2013, 87, 235434:1-235434:6.

15. Ramasubramaniam, A.; Naveh, D.; Towe, E. Tunable band gaps in bilayer transition-metal dichalcogenides. Phys. Rev. B 2011, 84, 205325:1-205325:10.

16. Liu, Q.; Li, L.; Li, Y.; Gao, Z.; Chen, Z.; Lu, J. Tuning Electronic Structure of Bilayer $\mathrm{MoS}_{2}$ by Vertical Electric Field: A First-Principles Investigation. J. Phys. Chem. C 2012, 116, 21556-21562.

17. Enyashin, A.N.; Bar-Sadan, M.; Houben, L.; Seifert, G. Line Defects in Molybdenum Disulfide Layers. J. Phys. Chem. C 2013, 117, 10842-10848.

18. Van der Zande, A.M.; Huang, P.Y.; Chenet, D.A.; Berkelbach, T.C.; You, Y.; Lee, G.H.; Heinz, T.F.; Reichman, D.R.; Muller, D.A.; Hone, J.C. Grains and grain boundaries in highly crystalline monolayer molybdenum disulphide. Nat. Mater. 2013, 12, 554-561.

19. Ghorbani-Asl, M.; Enyashin, A.N.; Kuc, A.; Seifert, G.; Heine, T. Defect-induced conductivity anisotropy in $\mathrm{MoS}_{2}$ monolayers. Phys. Rev. B 2013, 88, 245440:1-245440:7. 
20. Goyenola, C.; Gueorguiev, G.K.; Stafström, S. Fullerene-like $\mathrm{CS}_{x}$ : A first-principles study of synthetic growth. Chem. Phys. Lett. 2011, 506, 86-91.

21. Hellgren, N.; Berlind, T.; Gueorguiev, G.K.; Johansson, M.P.; Stafström, S.; Hultman, L. Fullerene-like B-C-N thin films: A computational and experimental study. Mater. Sci. Eng. B 2004, 113, 242-247.

22. Tenne, R.; Margulis, L.; Genut, M.; Hodes, G. Polyhedral and cylindrical structures of tungsten disulfide. Nature 1992, 360, 444-446.

23. Margulis, L.; Salitra, G.; Tenne, R.; Talianker, M. Nested Fullerene-like Structures. Nature 1993, 365, 113-114.

24. Seifert, G.; Terrones, H.; Terrones, M.; Jungnickel, G.; Frauenheim, T. Structure and electronic properties of $\mathrm{MoS}_{2}$ nanotubes. Phys. Rev. Lett. 2000, 85, 146-149.

25. Seifert, G.; Kohler, T.; Tenne, R. Stability of Metal Chalcogenide Nanotubes. J. Phys. Chem. B 2002, 106, 2497-2501.

26. Kis, A.; Mihailovic, D.; Remskar, M.; Mrzel, A.; Jesih, A.; Piwonski, I.; Kulik, A.J.; Benoit, W.; Forro, L. Shear and Young's moduli of $\mathrm{MoS}_{2}$ nanotube ropes. Adv. Mater. 2003, 15, 733-736.

27. Milosevic, I.; Nikolic, B.; Dobardzic, E.; Damnjanovic, M.; Popov, I.; Seifert, G. Electronic properties and optical spectra of $\mathrm{MoS}_{2}$ and $\mathrm{WS}_{2}$ nanotubes. Phys. Rev. B 2007, 76, 233414.

28. Tenne, R.; Redlich, M. Recent progress in the research of inorganic fullerene-like nanoparticles and inorganic nanotubes. Chem. Soc. Rev. 2010, 39, 1423-1434.

29. Zibouche, N.; Kuc, A.; Heine, T. From layers to nanotubes: Transition metal disulfides $\mathrm{TMS}_{2}$. Eur. Phys. J. B 2012, 85, 1-7.

30. Lorenz, T.; Teich, D.; Joswig, J.O.; Seifert, G. Theoretical Study of the Mechanical Behavior of Individual $\mathrm{TiS}_{2}$ and $\mathrm{MoS}_{2}$ Nanotubes. J. Phys. Chem. C 2012, 116, 11714-11721.

31. Mattheis, L.F. Energy-Bands For $2 \mathrm{H}-\mathrm{NbSe}_{2}$ And $2 \mathrm{H}-\mathrm{MoS}_{2}$. Phys. Rev. Lett. 1973, 30, 784-787.

32. Drummond, C.; Alcantar, N.; Israelachvili, J.; Tenne, R.; Golan, Y. Microtribology and friction-induced material transfer in $\mathrm{WS}_{2}$ nanoparticle additives. Adv. Funct. Mater. 2001, $11,348-354$.

33. Stefanov, M.; Enyashin, A.N.; Heine, T.; Seifert, G. Nanolubrication: How Do $\mathrm{MoS}_{2}$-Based Nanostructures Lubricate? J. Phys. Chem. C 2008, 112, 17764-17767.

34. Kaplan-Ashiri, I.; Cohen, S.R.; Gartsman, K.; Rosentsveig, R.; Seifert, G.; Tenne, R. Mechanical behavior of individual $\mathrm{WS}_{2}$ nanotubes. J. Mater. Res. 2004, 19, 454-459.

35. Kaplan-Ashiri, I.; Cohen, S.R.; Gartsman, K.; Rosentsveig, R.; Ivanovskaya, V.; Heine, T.; Seifert, G.; Wagner, H.D.; Tenne, R. Direct tensile tests of individual $\mathrm{WS}_{2}$ nanotubes. In Proceedings of the Pricm 5: The Fifth Pacific Rim International Conference on Advanced Materials, Pts 1-5, Beijing, China, 2-5 November 2004; Volume 475-479, pp. 4097-4102. 
36. Kaplan-Ashiri, I.; Cohen, S.R.; Gartsman, K.; Rosentsveig, R.; Ivanovskaya, V.V.; Heine, T.; Seifert, G.; Wagner, H.D.; Tenne, R. On the Mechanical Behavior of Tungsten Disulfide Nanotubes under Axial Tension and Compression. Proc. Natl. Acad. Sci. USA 2006, 103, 523-528.

37. Tang, D.M.; Wei, X.; Wang, M.S.; Kawamoto, N.; Bando, Y.; Zhi, C.; Mitome, M.; Zak, A.; Tenne, R.; Golberg, D. Revealing the Anomalous Tensile Properties of $\mathrm{WS}_{2}$ Nanotubes by in Situ Transmission Electron Microscopy. Nano Lett. 2013, 13, 1034-1040.

38. Ivanovskaya, V.V.; Heine, T.; Gemming, S.; Seifert, G. Structure, stability and electronic properties of composite $\mathrm{Mo}_{1-x} \mathrm{Nb}_{x} \mathrm{~S}_{2}$ nanotubes. Phys. Stat. Solidi B 2006, 243, 1757-1764.

39. Yun, W.S.; Han, S.W.; Hong, S.C.; Kim, I.G.; Lee, J.D. Thickness and strain effects on electronic structures of transition metal dichalcogenides: $2 H-\mathrm{MX}_{2}$ semiconductors $(\mathrm{M}=\mathrm{Mo}$, $\mathrm{W} ; \mathrm{X}=\mathrm{S}, \mathrm{Se}, \mathrm{Te})$. Phys. Rev. B 2012, 85, 033305.

40. Scalise, E.; Houssa, M.; Pourtois, G.; Afanas'ev, V.; Stesmans, A. Strain-induced semiconductor to metal transition in the two-dimensional honeycomb structure of $\mathrm{MoS}_{2}$. Nano Res. 2012, 5, 43-48.

41. Ghorbani-Asl, M.; Zibouche, N.; Wahiduzzaman, M.; Oliveira, A.F.; Kuc, A.; Heine, T. Electromechanics in $\mathrm{MoS}_{2}$ and $\mathrm{WS}_{2}$ : Nanotubes vs. monolayers. $\quad$ Sci. Rep. 2013, 3, 2961:1-2961:8.

42. Dovesi, R.; Saunders, V.R.; Roetti, R.; Orlando, R.; Zicovich-Wilson, C.M.; Pascale, F.; Civalleri, B.; Doll, K.; Harrison, N.M.; Bush, I.J.; et al. CRYSTAL09 User's Manual; University of Torino: Torino, Italy, 2009.

43. Perdew, J.P.; Burke, K.; Ernzerhof, M. Generalized gradient approximation made simple. Phys. Rev. Lett. 1996, 77, 3865-3868.

44. Cora, F.; Patel, A.; Harrison, N.M.; Roetti, C.; Catlow, C.R.A. An ab initio Hartree-Fock study of alpha- $\mathrm{MoO}_{3}$. J. Mater. Chem. 1997, 7, 959.

45. Cora, F.; Patel, A.; Harrison, N.M.; Dovesi, R.; Catlow, C.R.A. An ab initio Hartree-Fock study of the cubic and tetragonal phases of bulk tungsten trioxide. J. Am. Chem. Soc. 1996, $118,12174-12182$.

46. Monkhorst, H.J.; Pack, J.D. Special Points For Brillouin-Zone Integrations. Phys. Rev. B 1976, 13, doi:10.1103/PhysRevB.13.5188.

47. Seifert, G.; Porezag, D.; Frauenheim, T. Calculations of molecules, clusters, and solids with a simplified LCAO-DFT-LDA scheme. Int. J. Quantum Chem. 1996, 58, 185-192.

48. Oliveira, A.F.; Seifert, G.; Heine, T.; Duarte, H.A. Density-Functional Based Tight-Binding: An Approximate DFT Method. J. Br. Chem. Soc. 2009, 20, 1193-1205.

49. Wahiduzzaman, M.; Oliveira, A.F.; Philipsen, P.; Zhechkov, L.; van Lenthe, E.; Witek, A.H.; Heine, T. DFTB Parameters for the Periodic Table: Part 1, Electronic Structure. J. Chem. Theory Comput. 2013, 9, 4006-4017.

50. Di Carlo, A.; Gheorghe, M.; Bolognesi, A.; Lugli, P.; Sternberg, M.; Seifert, G.; Frauenheim, T. Molecular Devices Simulations Based on Density Functional Tight-Binding. J. Comput. Electron. 2002, 1, 109-112. 
51. Datta, S. Quantum Transport: Atom to Transistor, 2 ed.; Cambridge University Press: Cambridge, UK; New York, NY, USA, 2005.

52. Ghorbani-Asl, M.; Juarez-Mosqueda, R.; Kuc, A.; Heine, T. Efficient Quantum Simulations of Metals within the $\Gamma$-Point Approximation: Application to Carbon and Inorganic 1D and 2D Materials. J. Chem. Theory Comput. 2012, 8, 2888-2895.

53. Li, T. Ideal strength and phonon instability in single-layer $\mathrm{MoS}_{2}$. Phys. Rev. B 2012, 85, 235407.

54. Zhang, D.B.; Dumitrica, T.; Seifert, G. Helical Nanotube Structures of $\mathrm{MoS}_{2}$ with Intrinsic Twisting: An Objective Molecular Dynamics Study. Phys. Rev. Lett. 2011, 107, doi:10.1103/PhysRevLett.107.109902.

55. Li, W.; Zhang, G.; Guo, M.; Zhang, Y.W. Strain-tunable electronic and transport properties of $\mathrm{MoS}_{2}$ nanotubes. Nano Res. 2014, accepted. 


\title{
Single- to Triple-Wall $\mathbf{W S}_{\mathbf{2}}$ Nanotubes Obtained by High-Power Plasma Ablation of $\mathbf{W S}_{2}$ Multiwall Nanotubes
}

\author{
Volker Brüser, Ronit Popovitz-Biro, Ana Albu-Yaron, Tommy Lorenz, Gotthard Seifert, \\ Reshef Tenne and Alla Zak
}

\begin{abstract}
The synthesis of inorganic nanotubes (INT) from layered compounds of a small size $(<10 \mathrm{~nm}$ in diameter) and number of layers $(<4)$ is not a trivial task. Calculations based on density functional tight-binding theory (DFTB) predict that under highly exergonic conditions, the reaction could be driven into a "window" of (meta-) stability, where 1-3-layer nanotubes will be formed. Indeed, in this study, single- to triple-wall $\mathrm{WS}_{2}$ nanotubes with a diameter of 3-7 nm and a length of 20-100 nm were produced by high-power plasma irradiation of multiwall $\mathrm{WS}_{2}$ nanotubes. As target materials, plane crystals $(2 \mathrm{H})$, quasi spherical nanoparticles (IF) and multiwall, 20-30 layers, $\mathrm{WS}_{2}$ nanotubes were assessed. Surprisingly, only INT-WS 2 treated by plasma resulted in very small, and of a few layers, "daughter" nanotubules. The daughter nanotubes occur mostly attached to the outer surface of the predecessor, i.e., the multiwall "mother" nanotubes. They appear having either a common growth axis with the multiwall nanotube or tilted by approximately $30^{\circ}$ or $60^{\circ}$ with respect to its axis. This suggests that the daughter nanotubes are generated by exfoliation along specific crystallographic directions. A growth mechanism for the daughter nanotubes is proposed. High resolution transmission and scanning electron microscopy (HRTEM/HRSEM) analyses revealed the distinctive nanoscale structures and helped elucidating their growth mechanism.
\end{abstract}

Reprinted from Inorganics. Cite as: Brüser, V.; Popovitz-Biro, R.; Albu-Yaron, A.; Lorenz, T.; Seifert, G.; Tenne, R.; Zak, A. Single- to Triple-Wall WS2 Nanotubes Obtained by High-Power Plasma Ablation of $\mathrm{WS}_{2}$ Multiwall Nanotubes. Inorganics 2014, 2, 177-190.

\section{Introduction}

Multiwall inorganic nanotubes of $\mathrm{WS}_{2}\left(\mathrm{INT}-\mathrm{WS}_{2}\right)$ were discovered in 1992 [1], and the route for their scaled-up synthesis was developed in 2009 [2]. Together with BN [3] and $\mathrm{MoS}_{2}$ [4,5], they probably constitute the most investigated kind of inorganic nanotubes from layered compounds. The crystalline and electronic structure of INT has been studied in great detail [6-8]. In particular, calculations have shown that multiwall $\mathrm{WS}_{2}\left(\mathrm{MoS}_{2}\right)$ nanotubes become more stable than the respective nanosheets at a threshold outer diameter of about 15 to $20 \mathrm{~nm}$ and being made up of at least 5-10 layers [9]. Indeed, many of the high-temperature (above $700{ }^{\circ} \mathrm{C}$ ) synthetic strategies ended up in multiwall nanotubes exhibiting a high-crystalline order, which agree quite well with the predicted sizes $[2,10,11]$.

Nonetheless, these conditions are not sufficiently exergonic to drive the reaction into windows of (meta-) stability far enough from equilibrium, where 1-3-layer nanotubes could be formed. It was shown in the past that reactions carried out under highly exergonic conditions, like laser ablation [12], for example, can yield closed-cage $\mathrm{MoS}_{2}$ nanoparticles having a small size and number of layers. Calculations based on density functional tight-binding theory (DFTB) [9] (see Figure 1) present the 
energy-per-atom of nanotubes as a function of the number of atoms in the unit length (unit cell), N $\mathrm{Not}_{\text {, }}$ and for different number of layers $(k=1-4)$. They are compared with nanostripes (nanoribbons) of the same number of atoms. For the sake of simplicity, the calculations were carried out for $\mathrm{MoS}_{2}$, which is structurally analogous to $\mathrm{WS}_{2}$. It is noticed that the energy-per-atom increases with a decreasing number of atoms for both the nanostripes and the nanotubes, but for different reasons. The energy-per-atom for the nanostripes increases, due to edge effects, i.e., the abundance of rim atoms with dangling bonds. On the other hand, the nanotubes become less stable at a smaller radius of curvature, due to the increasing elastic energy of folding. In addition, the folding energy increases more steeply for the nanotubes than the energy of the nanoribbons as the number of atoms shrinks. Consequently, smaller diameter nanotubes become less stable than the straight nanostripes to the left of the cross-over point (stability threshold) of the two curves. While the cross-over point itself moves to the left as the number of layers decreases, the corresponding threshold energy-per-atom rapidly increases (becomes less negative), particularly for nanotubes with three layers and below. It is therefore clear that the generation of nanotubes of a small size and number of layers $(k<4)$ requires highly exergonic conditions, which is the subject of the present work.

Figure 1. The calculated energy-per-atom for $\mathrm{MoS}_{2}$ nanotubes and nanostripes with 1-4 walls as a function of the number of atoms in the tube unit cell, $N_{\text {tot. }}$.

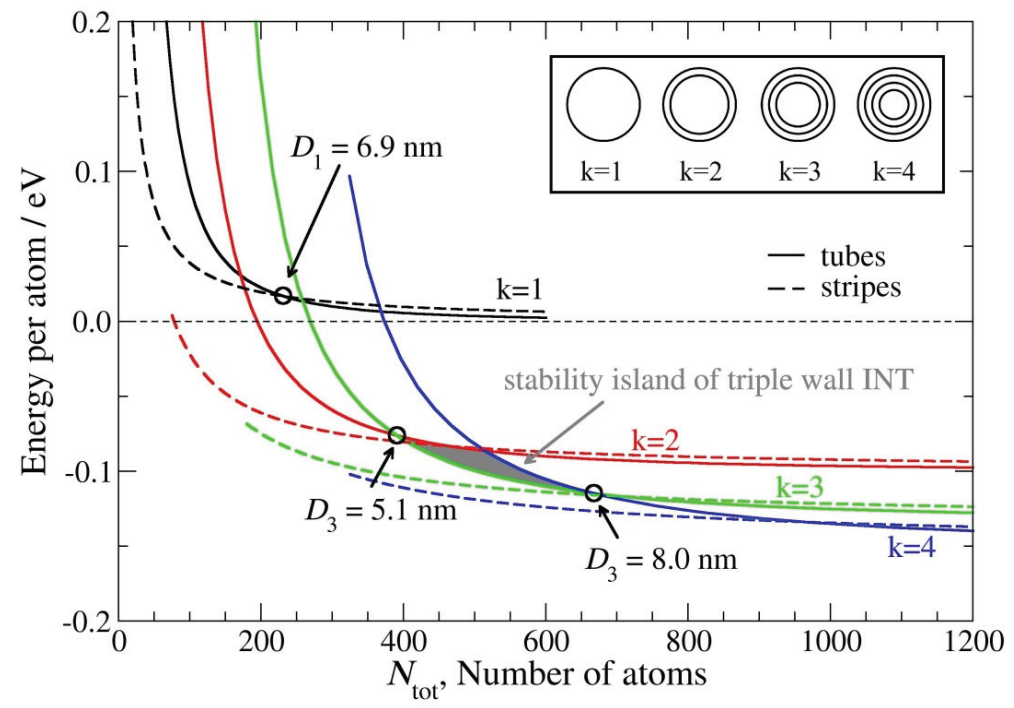

Interestingly, in the range of $\sim 390<N_{\text {tot }}<670$, which corresponds to nanotubes with outer diameters of $5.1 \mathrm{~nm}<D_{3}<8.0 \mathrm{~nm}$, the triple layer nanotubes are more stable than nanotubes with $k=2$ and $k=4$ (see Figure 1). The diameter $\left(D_{k}\right)$ represents here the outer diameters of the nanotubes with $k$ shells. This theoretical prediction is in agreement with experimental results presented in this work: the majority of the daughter nanotubes were triple-walled. Note that nanotubes with the same (outer) diameters, but different number of shells, have consequently a different (total) number of 
atoms. Thus, a single-wall tube with a larger diameter may have less atoms than triple-walled tubes of a smaller diameter.

A similar situation has been encountered with the stability window of $\mathrm{MoS}_{2}$ nanotetrahedra and nanooctahedra consisting of 2-4 layers. These nanostructures were proposed first in $[13,14]$ and realized in $[15,16]$. Indeed, $\mathrm{MoS}_{2}$ nanooctahedra/nanotetrahedra were obtained by rapid quenching of laser- [15-18] or solar- [19] ablated $\mathrm{MoS}_{2}$ soot or by an arc-discharge process [20]. It can, therefore, be concluded that highly exergonic reaction conditions and rapid quenching of the nanoclusters can access (meta-) stability windows, which favor new nanotubes that are not reachable by the conventional thermally-driven synthesis at $<1000^{\circ} \mathrm{C}$.

\section{Results and Discussion}

In the present work, 1-3-layer $\mathrm{WS}_{2}$ nanotubes with a diameter of 3-7 nm and a length of 20-100 nm were produced by applying inductively coupled radio-frequency plasma irradiation on multiwall INT-WS 2 .

\subsection{Scanning and Transmission Electron Microscopy Analysis}

Typical scanning and transmission electron microscopy images of a pristine (untreated) multiwall $\mathrm{WS}_{2}$ ("mother") nanotube are presented in Figure 2a,b, respectively. The majority of the predecessor INT was 5-20 microns in length and 30-120 nm in diameter.

The HRTEM images in Figure 3a,b display the range of daughter nanotubes obtained by plasma-treating of the multiwall $\mathrm{WS}_{2}$ nanotubes at $600 \mathrm{~W}$ for $40 \mathrm{~min}$ : tiny daughter nanotubes adjacent to the outer surface (Figure $3 \mathrm{a}$ ) of the mother nanotube and a few isolated daughter nanotubes (Figure $3 \mathrm{~b}$ ). The amount of such daughter nanotubes increased with the treatment time from 10 to $40 \mathrm{~min}$ at $400 \mathrm{~W}$ plasma power. The extension of the plasma treatment time to $80 \mathrm{~min}$ did not reveal any additional improvement; however, the increase of plasma power from 400 to $600 \mathrm{~W}$ resulted in a sharp increase in the amount of the daughter nanotubes.

At $600 \mathrm{~W}$ and $40 \mathrm{~min}$ of treatment, a rough statistical estimate shows that daughter nanotubes were attached to about $80 \%$ of the plasma treated multiwall nanotubes. In comparison, only $10-20 \%$ of the multiwall $\mathrm{WS}_{2}$ nanotubes were covered with daughter nanotubes by a $400-\mathrm{W}$ plasma treatment. Some nanostructures could be better described as nanoscrolls. However, the majority of the daughter nanostructures are nanotubes, having at least one perfectly closed layer. Future work will be focused on devising this technique to increase the yield of a single- to a few-layer nanotubes of $\mathrm{WS}_{2}$ or other INT, as well. Indeed, by irradiating $\mathrm{MoS}_{2}$ powder with a focused solar beam, single-wall $\mathrm{MoS}_{2}$ was rarely observed in the processed powder [21], which confirms that highly exergonic conditions produced by focused solar (laser) ablation may lead to the production of single-wall nanotubes of this kind. 
Figure 2. (a) SEM and (b) TEM micrograph of a pristine multiwall $\mathrm{WS}_{2}$ nanotube.

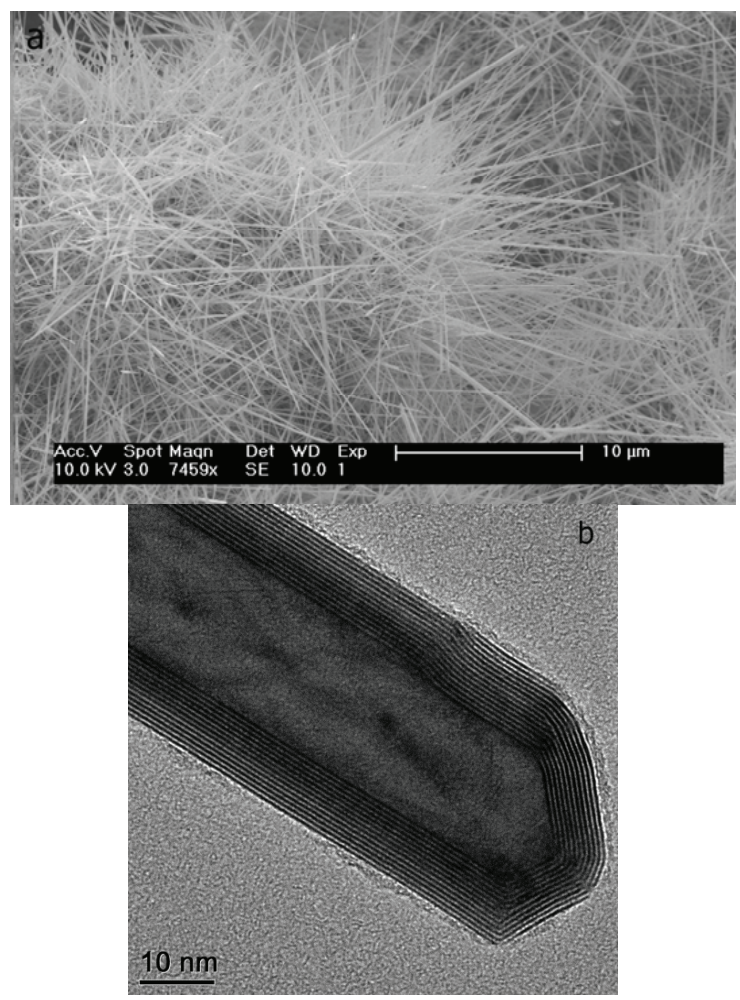

Figure 3. TEM images of daughter $\mathrm{WS}_{2}$ nanotubes obtained by plasma ablation of multiwall inorganic nanotube (INT)-WS 2 at $600 \mathrm{~W}$ for $40 \mathrm{~min}$ : (a) A large number of daughter nanotubes next to a treated multiwall nanotube; (b) A group of daughter nanotubes isolated from plasma-treated multiwall $\mathrm{WS}_{2}$ nanotubes by sonication.

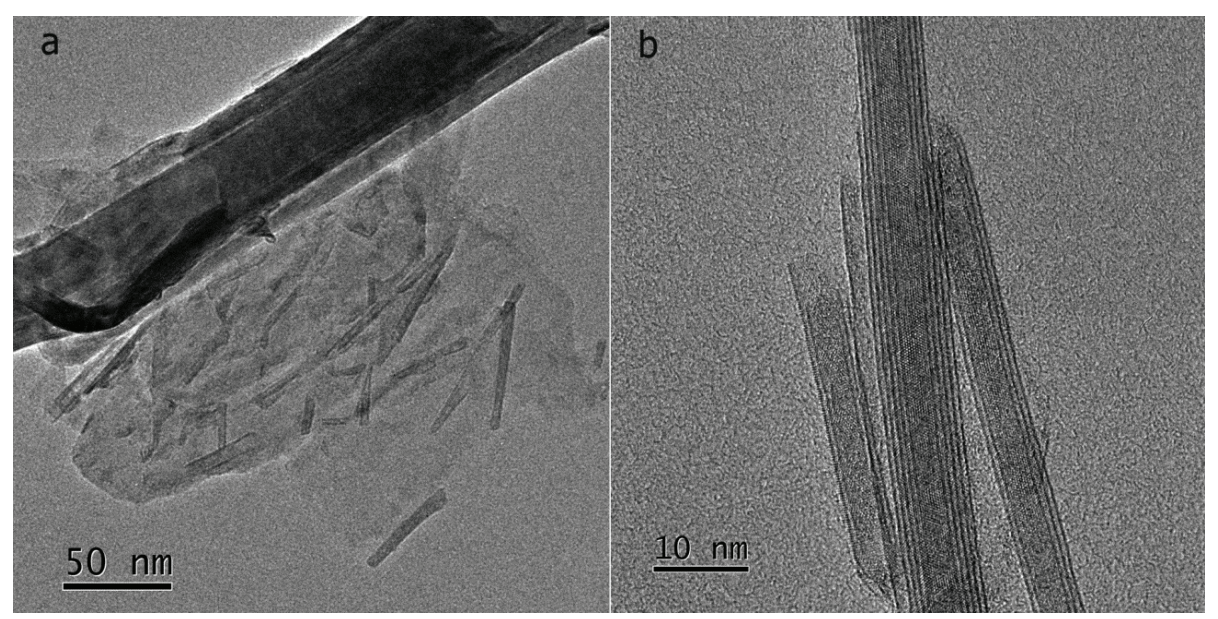


Moreover, many daughter nanotubes were found attached and being tilted at $c a .30$ or $60^{\circ}$ with respect to the mother nanotube axis (see the white arrows in Figure 4a,b). However, some daughter nanotubes were found to be attached, having a common growth axis with the multiwall nanotube (see the black arrow in Figure 4b).

Figure 4. (a) TEM images of the daughter nanotubes tethered to the surface and tilted at approximately 30 or $60^{\circ}$ with respect to the mother nanotube growth axis (white arrows);

(b) TEM image of a daughter nanotube with growth axis parallel to the mother nanotube (black arrows).
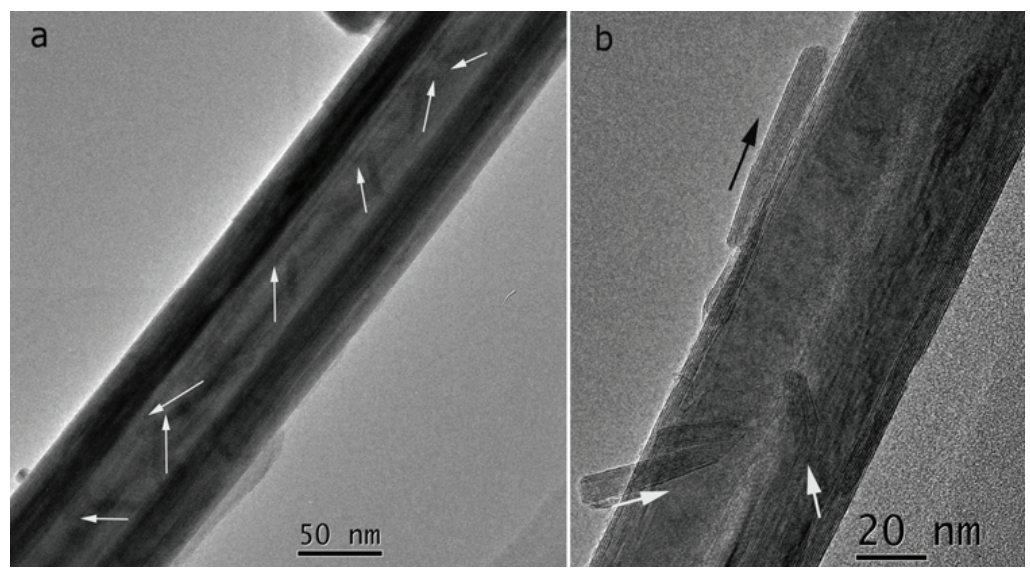

These observations suggest that the small nanotubes were exfoliated by unzipping the outer walls of the mother nanotubes along specific crystallographic directions. In addition to nanotubes/nanoscrolls, a few layers-thick $\mathrm{WS}_{2}$ nanoplatelets of typical sizes in the range of the nanotubes' length, i.e., $50-100 \mathrm{~nm}$, were also observed.

In an attempt to separate the daughter nanotubes from the mother nanotubes, the plasma-ablated $\mathrm{WS}_{2}$ nanotube powder was ultrasonically treated in ethanol for $10 \mathrm{~min}$. The high-resolution TEM (HRTEM) images in Figure 5 (see also Figure 3b) clearly depict the daughter nanotubes being more easily observed after detachment from the predecessor nanotubes.

Rough statistical analysis revealed that the interlayer distance in most daughter nanotubes varied between 6.3-6.5 $\AA$ (see Figure 5a), which is larger than the interlayer spacing of $2 \mathrm{H}-\mathrm{WS}_{2}$ $\left(6.23 \AA / 2\right.$-Theta $\left.=14.32^{\circ}\right)$ and multiwall nanotubes $\left(6.31 \AA / 2\right.$-Theta $\left.=14.13^{\circ}\right)[2]$. This observation suggests that the daughter nanotubes were not fully relaxed during the growth process and that the annealing of the sample could possibly lead to further structural relaxation. The Fourier (FFT) analysis (see the inset in Figure 5b) of the area framed by the square shows that the nanotube is chiral with a helical angle of six degrees. Once daughter nanotubes are observed in larger yields, techniques like ultracentrifugation could be used to separate them according to the number of layers and the length. 
Figure 5. (a) HRTEM images of a two-three-layer nanotube with a non-uniform diameter after detachment from the large $\mathrm{WS}_{2}$ multiwall nanotube; (b) Another three-layer daughter nanotube after detachment. The Fourier (FFT) analysis (see the inset) of the area framed by the square shows that the nanotube is chiral with a helical angle of six degrees.

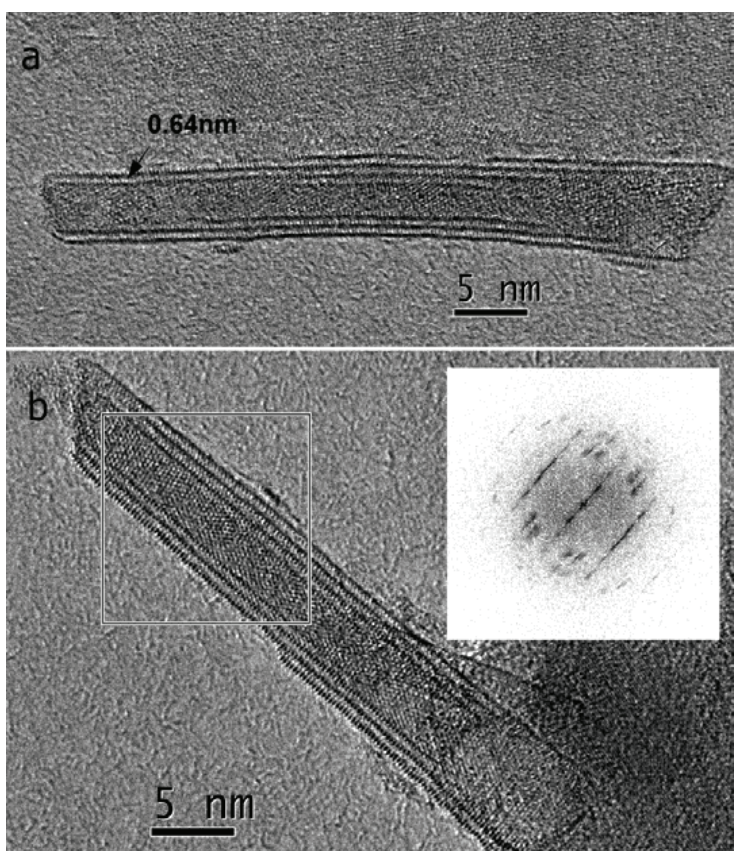

Energy dispersive X-ray analysis (EDS) within the TEM (not shown) confirmed that the nanotubes are made solely of tungsten and sulfur. Negligible traces of oxygen were found, which could be mainly attributed to surface impurities.

In a separate series of experiments, several powders of different microcrystalline layered materials, including $2 \mathrm{H}-\mathrm{WS}_{2}, 2 \mathrm{H}-\mathrm{MoS}_{2}$ and $2 \mathrm{H}-\mathrm{NbS}_{2}$, and also the respective diselenides, received a similar plasma treatment. No daughter nanotubes were found in these treated samples, whatsoever. A few layers-thick $W_{2}$ nanoplatelets with typical sizes in the range of the nanotubes $(50-100 \mathrm{~nm})$ were nevertheless abundant in plasma-treated $2 \mathrm{H}-\mathrm{WS}_{2}$ powder. Furthermore, the $\mathrm{NbSe}_{2}$ powder turned out to be unstable under the plasma treatment conditions. Plasma treatment $(400 \mathrm{~W})$ of fullerene-like $\mathrm{WS}_{2}$ nanoparticles with hollow cage structure (inorganic fullerene-like (IF-WS $\mathrm{S}_{2}$ nanoparticles) resulted in a few layers exfoliation and a few small-sized ("daughter") fullerene-like nanoparticles or nanotubes (see Figure 6). The daughter IF-WS 2 nanoparticles are reminiscent of the arc-discharge produced IF-MoS 2 nanoparticles [20]. In concluding this large series of experiments, it is possible to state that only plasma irradiation of multiwall $\mathrm{WS}_{2}$ nanotubes yielded daughter nanotubes in a reproducible fashion. 
Figure 6. TEM image of the attached daughter single wall fullerene-like nanoparticles generated by plasma treatment of multiwall fullerene-like $\mathrm{WS}_{2}$ nanoparticles.

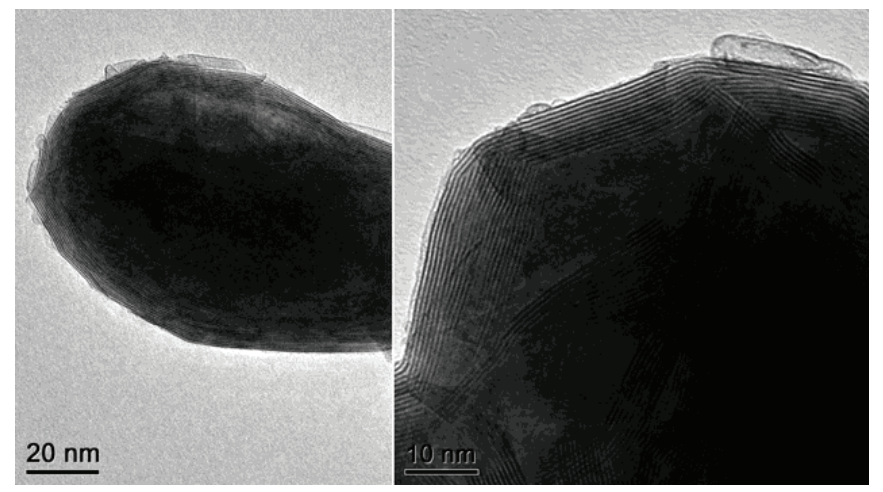

Single-wall carbon nanotubes can be obtained in large quantities, e.g., via the arc-discharge technique [22]. Given the interlayer distance of $3.4 \AA$ in graphite, the monoatomic graphene plane can be closed into nanotubes of a diameter smaller than $0.5 \mathrm{~nm}[23,24]$. On the other hand, the $\mathrm{WS}_{2}$ $\left(\mathrm{MoS}_{2}\right)$ layer consists of six-fold bonded tungsten (molybdenum) atoms sandwiched between two sub-layers of three-fold bonded sulfur atoms. This makes the $\mathrm{WS}_{2}$ layers pretty rigid, with interlayer spacing of $6.23 \AA$; it is no wonder that the elastic energy for $\mathrm{WS}_{2}\left(\mathrm{MoS}_{2}\right)$ nanotube formation is appreciably larger than that of graphitic carbon. If one takes the elastic energy threshold for folding to be $0.05 \mathrm{eV} /$ atom, the calculated diameter of a single-wall carbon nanotube is between one and $1.2 \mathrm{~nm}[25]$, and that of a single-wall $\mathrm{MoS}_{2}$ should be $6.2 \mathrm{~nm}[9,26]$. Therefore, the diameters of the daughter $\mathrm{WS}_{2}$ nanotubes observed in the current series of experiments reconcile very well with the previous calculations.

\subsection{Growth Mechanism}

It is hypothesized that the formation of the daughter nanotube occurs through a strong interaction of the highly energetic plasma, used in this work, with a point or line defect on the outer surface of the mother nanotube, leading to rapid unzipping and exfoliation of 1-3 layers-thick $\mathrm{WS}_{2}$ fragments. One way for the exfoliated nanosheets to release the large elastic strain and fold into a nanotube is through an "inverted umbrella" reaction, which is the manifestation of the "Walden inversion" typical of a nucleophilic attack of a stereoisomer by an electron-rich moiety [27].

In an effort to understand the mechanism of formation of these daughter nanotubes, additional HRSEM analysis of the plasma-treated nanotubes was undertaken. The HRSEM in Figure 7a reveals a reversely revolved nanoscroll of $\sim 20 \mathrm{~nm}$ in diameter attached to the surface of the mother nanotube. Furthermore, a clearly observed step defect or dark contrast on the mother nanotube beneath the daughter nanoscroll is reminiscent of the exfoliation process of the $\mathrm{WS}_{2}$ patch. Unfortunately, the resolution of the SEM did not permit viewing the smaller (3-7 $\mathrm{nm}$ ) daughter nanotubes. 
Figure 7. (a) HRSEM micrograph of a daughter nanoscroll attached to a large nanotube; (b) (I) HRTEM of a two-layer daughter nanoscroll viewed head-on along its axis and attached to a large multiwall nanotube; (II) an initial scrolling stage of an exfoliated single layer and three (III) layers before the formation of the nanotube; (c) HRSEM image of a daughter nanotube attached to a mother INT; and (d) HRTEM images revealing the folding of $\mathrm{WS}_{2}$ nanosheets to form a daughter nanotube.

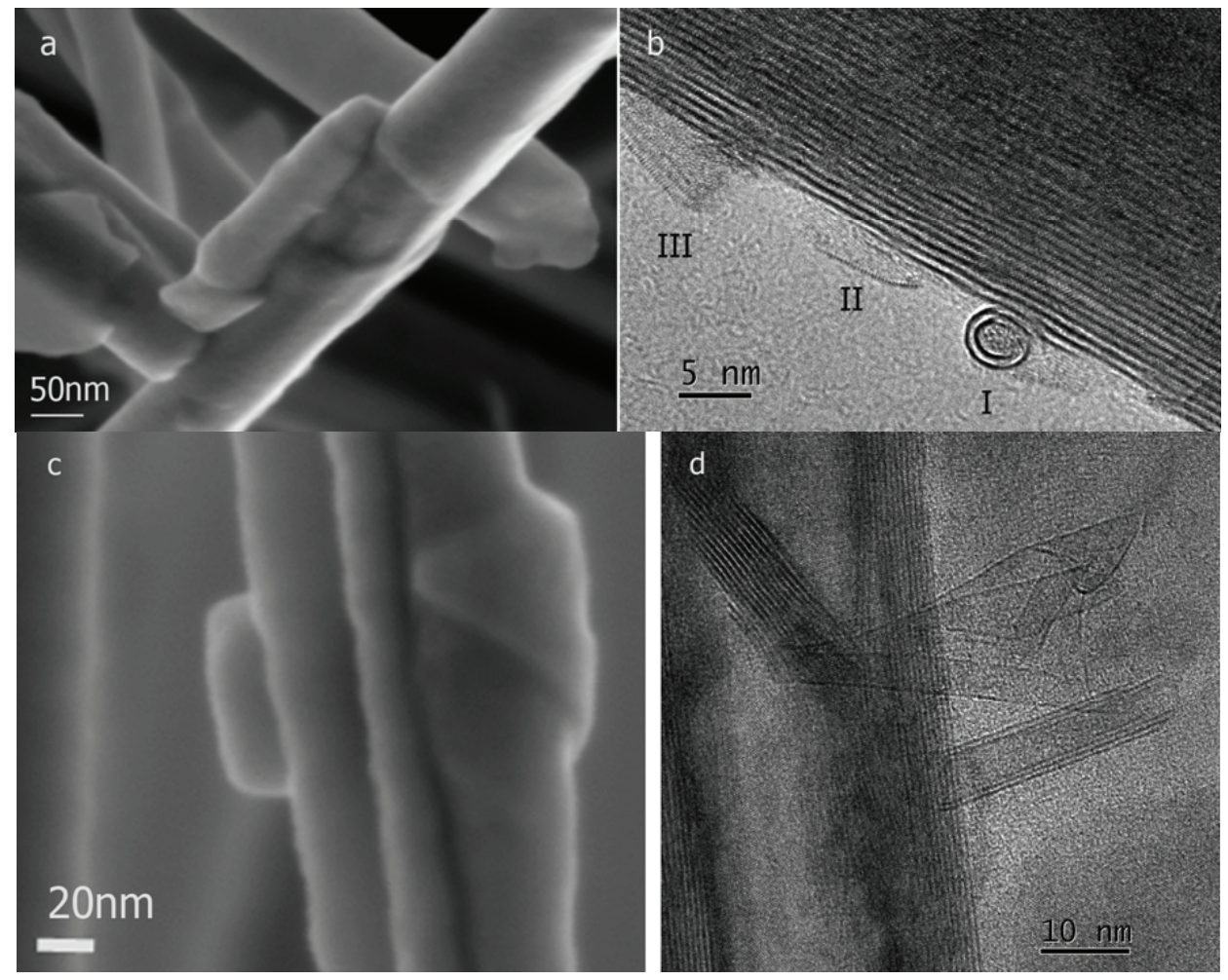

Nonetheless, this analysis suggests very strongly that the elastic strain of the exfoliated $\mathrm{WS}_{2}$ sheet produces oppositely revolved daughter nanotubes. Moreover, at better resolution, the HRTEM image in Figure 7b depicts a two-layer daughter nanoscroll viewed head-on along its axis marked by "I". Furthermore, an initial scrolling stage of an exfoliated single layer ("II") and three layers ("III") before the formation of the nanotube was also observed. In addition, Figure 7c,d shows an HRSEM image of a daughter nanotube attached to a mother nanotube and HRTEM images of a $\mathrm{WS}_{2}$ nanosheet in the process of folding to form a daughter nanotube.

A schematic model for the growth mechanism of the daughter nanotubes is depicted in Figure 8. This growth mechanism proposes that the fragments of the outermost (1-3) layers of the predecessor nanotubes were unzipped by the plasma treatment, exfoliated and folded into daughter nanotubes. The large excitation energies of the plasma together with the mechanical strain lead to a nanoscopic "Walden-type inversion" [27]. The reactive edges of the inverted layers induce further folding into daughter nanotubes with a smaller radius of curvature than the predecessor (mother) multiwall $\mathrm{WS}_{2}$ 
nanotube. Detachment of the 1-3 layers from the mother nanotube may also be followed by rotation and inclination, in this case, the axes of the mother and daughter nanotubes do not necessarily coincide or form a specific angle between them. In other cases the rapid quenching of the excess energy of the nanosheets does not permit them to fully close, which leads to nanoscrolls or to a nanotube with one closed wall and the others remaining unclosed. Nanoscrolls may also occur due to steric hindrance, where the plasma-induced exfoliated nanosheets released their energy without being able to undergo timely inversion.

Figure 8. Schematics of the proposed growth mechanism of the daughter nanotubes by plasma treatment of the multiwall mother nanotubes.

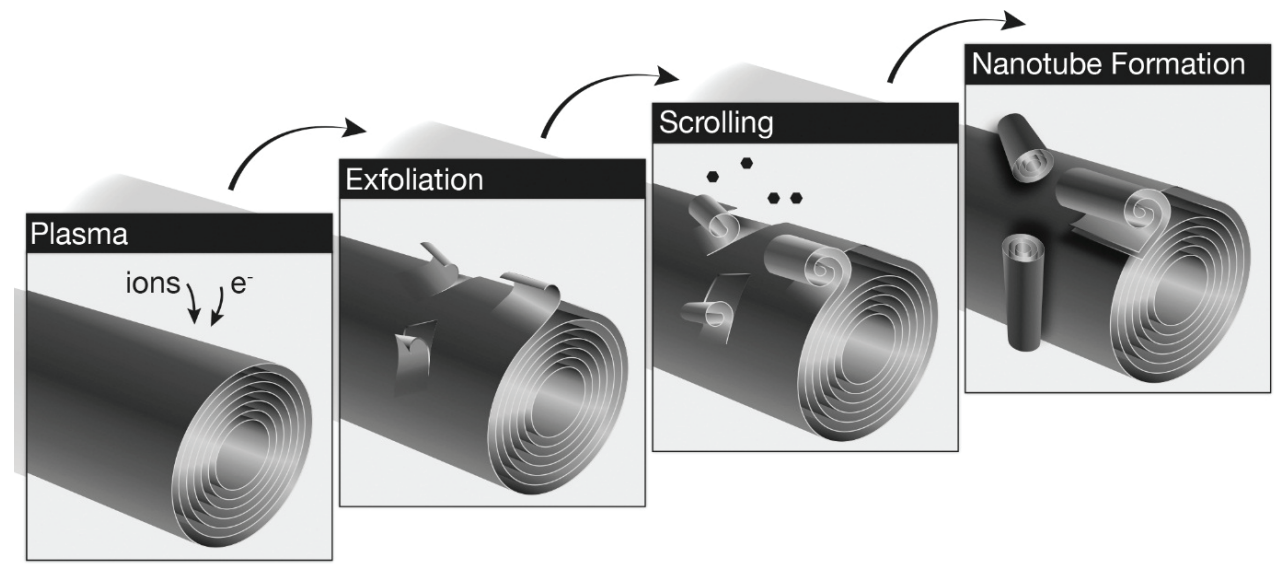

In a few cases, $\mathrm{WS}_{x}$ nanoclusters were observed adjacent to the daughter nanotubes (see Figure 9). These non-stoichiometric nanoclusters could be obtained by the condensation of tungsten and sulfur atoms or $\mathrm{WS}_{2}$ molecules from the vapor phase. In turn, the condensation of clusters onto the tube edges could lead to further elongation or even the growth of an extra layer on the daughter nanotube surface. Another plausible event is the condensation of the vapors into separate nanosheets, which, upon quenching, form isolated nanotubes.

The proposed mechanism is consistent with the data presented in this work. In order to shed light on the detailed growth mechanism of the (daughter) nanotubes and to control their length, diameter and the number of layers, future experiments will focus on the variation of the plasma treatment process, including the substrate temperature, pressure in the chamber, etc.

Since highly excited clusters of $\mathrm{WS}_{2}\left(\mathrm{MoS}_{2}\right)$ can be formed using arc-discharge and a variety of other techniques, high-power plasma ablation would possibly allow synthesizing a few-wall nanotubes under controlled conditions in higher yields. 
Figure 9. TEM images of nanoclusters surrounding daughter nanotubes. Presumably, the clusters were generated by the condensation of tungsten and sulfur atoms or $\mathrm{WS}_{2}$ molecular clusters from the vapor phase created by the plasma treatment of the multiwall $\mathrm{WS}_{2}$ nanotubes.

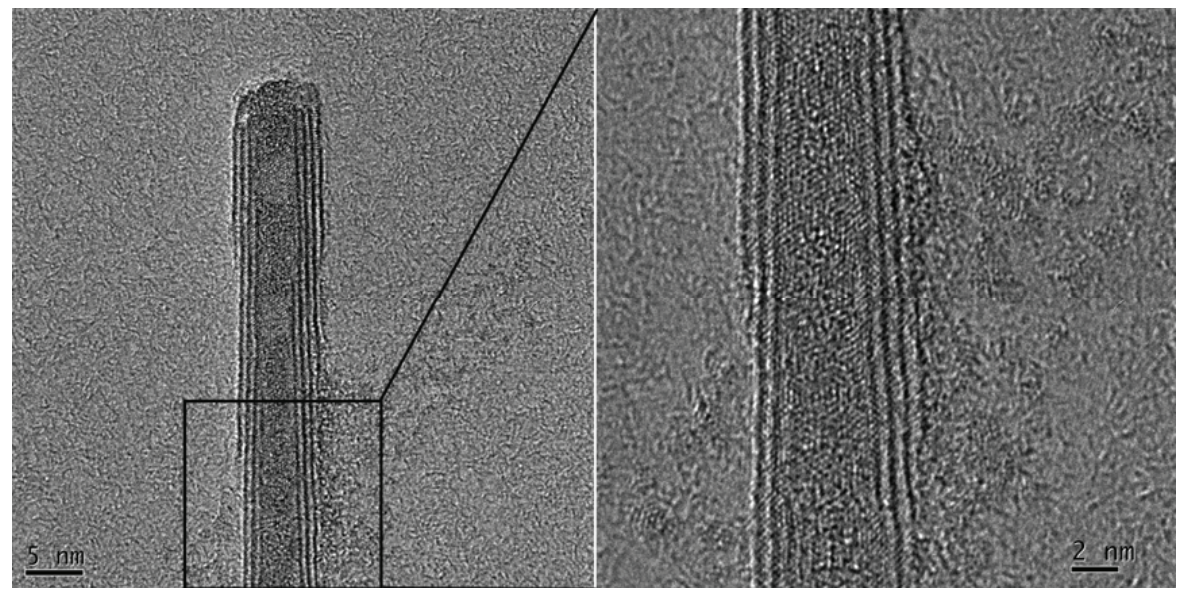

\section{Experimental Section}

\subsection{Plasma Treatment}

The schematic drawing and photograph of the experimental set-up for the inductively coupled radio-frequency plasma irradiation $(27.12 \mathrm{MHz})$ [28] of the multiwall $\mathrm{WS}_{2}$ nanotubes is depicted in Figure 10.

In these experiments, non-thermal plasma with electrons, atoms and ions, having different temperatures each, was used to irradiate powders of multiwall $\mathrm{WS}_{2}$ nanotubes (INT-WS 2 ), inorganic fullerene-like (IF) quasi spherical nanoparticles and different transition metal dichalcogenides microcrystalline $2 \mathrm{H}$-platelets. A plasma power in the range of 400-600 W was applied for 5, 10, 20, 40 and $80 \mathrm{~min}$. The electron temperature in these experiments was in the range of $1.7 \times 10^{4}-2.3 \times 10^{4} \mathrm{~K}(1.5-2 \mathrm{eV})$, and the electron density was in the range of $\sim 10^{12} / \mathrm{cm}^{3}$ [29].

The argon gas pressure was $10 \mathrm{~Pa}$, and the flow speed of the Ar gas was $30-35 \mathrm{~cm}^{3} / \mathrm{s}$, while the base pressure before the Ar gas was $10^{-4} \mathrm{~Pa}$. The temperature of the neutral Ar atoms and $\mathrm{Ar}^{+}$ions is approximately two orders of magnitude smaller than that of the electrons. The plasma energy impact on the substrate surfaces was $2.3 \mathrm{~W} / \mathrm{cm}^{2}$ at $400 \mathrm{~W}$ and $3.1 \mathrm{~W} / \mathrm{cm}^{2}$ at $600 \mathrm{~W}$ [30]. The plasma parameters, pressure and energy impact were constant over the treatment time. The temperature of the substrate increased with time and depended on the heat conductivity and the quality of the thermal contact between the powder and the susceptor. The nanoparticles temperature was different from that of the gas. It was influenced by a number of factors, including the electron and ion bombardment, electron-ion recombination, reaction enthalpy from the chemical surface reaction, energy loss by heat radiation and conduction. The temperature of the nanotubes could be estimated to be in the range of a few hundred degrees centigrade [31]. 
Figure 10. (a) Schematic representation and (b) Photograph of the experimental set-up for the plasma treatment of the multiwall $\mathrm{WS}_{2}$ nanotubes.

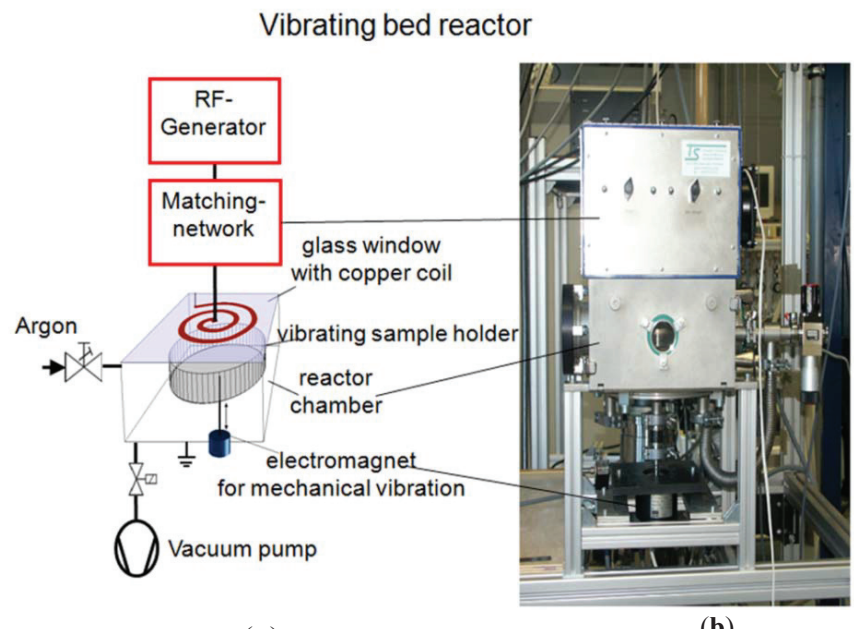

(a)

(b)

\subsection{Electron Microscopy}

The resulting samples were examined by transmission electron microscopy (TEM) (Philips CM120 operating at $120 \mathrm{kV}$, equipped with an energy dispersive X-ray spectroscopy (EDS) detector (EDAX Phoenix Microanalyzer) for chemical analysis). High-resolution TEM (HRTEM) (FEI Technai F30-UT, with a field-emission gun operating at $300 \mathrm{kV}$ ) and scanning transmission electron microscopy (STEM) (FEI Technai F20 operating at $200 \mathrm{kV}$ equipped with a high-angle annular dark field (HAADF) detector and EDS detector (EDAX-Phoenix Microanalyzer)) were also used. Complementary information was obtained by high-resolution scanning electron microscopy (HRSEM) (Zeiss Ultra model V55 and LEO model Supra 55VP equipped with an EDS detector (Oxford model INCA) and backscattering electron (BSE) detector).

\section{Conclusions}

In conclusion, WS2 nanotubes of 1-3 layers (“daughter nanotubes"), 20-100 nm-long and with diameters varying between 3-7 nm, were obtained by plasma treatment of multiwall nanotubes in inert atmosphere. The proposed growth mechanism of the daughter nanotubes involves the strong interaction of the plasma with point or line defects, causing unzipping and exfoliation of the outermost layers of the multiwall nanotube, the release of the elastic strain, followed by scrolling and closure into small nanoscrolls or nanotubes. Sublimation of W and $\mathrm{S}$ atoms, $\mathrm{WS}_{2}$ molecules and cluster formation could serve as an additional building material for daughter tube formation and extension. These few-layered $\mathrm{WS}_{2}$ nanotubes represent a locally stable, highly excited state of this solid. Being of such small dimensions, they should reveal a quantum confinement effect, as well as new optical, electrical and mechanical properties. Furthermore, these nanotubes can be suspended in different solvents and could possibly be of particular interest, e.g., for drug delivery. 


\section{Acknowledgments}

We gratefully acknowledge Gal Radovski for assisting with the HRSEM microscopy analysis. We gratefully acknowledge the support of the ERC project INTIF 226639, ITN MoWSeS 317451, the Israel Science Foundation, the COST action COINAPO MP0902, the FTA project of the Isr. Natl. NanoIntiative; the G.M.J. Schmidt Minerva Center for supramolecular chemistry; the Harold Perlman and the Irving and Azelle Waltcher Foundations, and the Irving and Cherna Moskowitz Center for Nano and Bio-Nano Imaging. R.T. is the Drake Family Chair in Nanotechnology and director of the Helen and Martin Kimmel Center for Nanoscale Science.

\section{Author Contributions}

V.B.: Plasma treatment; R.P.-B.: TEM analyses and discussion of the results; A.A.-Y.: TEM analysis and discussion of the results; T.L.: Theoretical calculations; G.S.: Theoretical calculations; R.T.: Analysis of the nanotubes, discussion and the dissemination of the results; A.Z.: Synthesis of inorganic multiwall $\mathrm{WS}_{2}$ nanotubes, TEM/SEM analyses, discussion of the plasma experiment, discussion the dissemination of the results.

\section{Conflicts of Interests}

The authors declare no conflict of interests.

\section{References}

1. Tenne, R.; Margulis, L.; Genut, M.; Hodes, G. Polyhedral and cylindrical structures of tungsten disulphide. Nature 1992, 360, 444-446.

2. Zak, A.; Sallacan-Ecker, L.; Margolin, A.; Genut, M.; Tenne, R. Insight into the growth mechanism of $\mathrm{WS}_{2}$ nanotubes in the scaled-up fluidized-bed reactor. Nano 2009, 4, 91-98.

3. Chopra, N.G.; Luyken, J.; Cherry, K.; Crespi, V.H.; Cohen, M.L.; Louie, S.G.; Zettl, A. Boron Nitride Nanotubes. Science 1995, 269, 966-967.

4. Feldman, Y.; Wasserman, E.; Srolovitz, D.J.; Tenne, R. High-rate, gas-phase growth of $\mathrm{MoS}_{2}$ nested inorganic fullerenes and nanotubes. Science 1995, 267, 222-225.

5. Remskar, M.; Skraba, Z.; Cleton, F.; Sanjines, R.; Levy, F. MoS2 as microtubes. Appl. Phys. Lett. 1996, 69, 351-353.

6. Rubio, A.; Corkill, J.F.; Cohen, M.L. Theory of graphitic boron nitride nanotubes. Phys. Rev. B 1994, 49, 5081-5084.

7. Seifert, G.; Terrones, H.; Terrones, M.; Jungnickel, G.; Frauenheim, T. Structure and electronic properties of $\mathrm{MoS}_{2}$ nanotubes. Phys. Rev. Lett. 2000, 85, 146-149.

8. Linnolahti, M.; Pakkanen, T.A. Quantum chemical treatment of large nanotubes via use of line group symmetry: Structural preferences of magnesium dichloride nanotubes. J. Phys. Chem. B 2006, 110, 4675-4678.

9. Seifert, G.; Köhler, T.; Tenne, R. Stability of metal chalcogenide nanotubes. J. Phys. Chem. B 2002, 106, 2497-2501. 
10. Therese, H.A.; Li, J.; Kolb, U.; Tremel, W. Facile large scale synthesis of $\mathrm{WS}_{2}$ nanotubes from $\mathrm{WO}_{3}$ nanorods prepared by a hydrothermal route. Solid State Sci. 2005, 7, 67-72.

11. Li, Y.D.; Li, X.L.; He, R.R.; Zhu, J.; Deng, Z.X. Artificial lamellar mesostructures to WS2 nanotubes. J. Am. Chem. Soc. 2002, 124, 1411-1416.

12. Sen, R.; Govindaraj, A.; Suenaga, K.; Suzuki, S.; Kataura, H.; Iijima, S.; Achiba, Y. Encapsulated and hollow closed-cage structures of $\mathrm{WS}_{2}$ and $\mathrm{MoS}_{2}$ prepared by laser ablation at 450-1050 ${ }^{\circ}$ C. Chem. Phys. Lett. 2001, 340, 242-248.

13. Margulis, L.; Salitra, G.; Tenne, R.; Talianker, M. Nested fullerene-like structures. Nature 1993, $365,113-114$.

14. Tenne, R. Doped and Heteroatom-Containing Fullerene-like Structures and Nanotubes. Adv. Mater. 1995, 7, 965-995.

15. Singh, D.M.D.J.; Pradeep, T.; Bhattacharjee, J.; Waghmare, U.V. Novel cage clusters of MoS(2) in the gas phase. J. Phys. Chem. A 2005, 109, 7339-7342.

16. Parilla, P.A.; Dillon, A.C.; Jones, K.M.; Riker, G.; Schulz, D.L.; Ginley, D.S.; Heben, M.J. The first true inorganic fullerenes? Nature 1999, 397, 114.

17. Enyashin, A.N.; Gemming, S.; Bar-Sadan, M.; Popovitz-Biro, R.; Hong, S.Y.; Prior, Y.; Tenne, R.; Seifert, G. Structure and stability of molybdenum sulfide fullerenes. Angew. Chem. Int. Ed. 2007, 46, 623-627.

18. Enyashin, A.N.; Bar-Sadan, M.; Sloan, J.; Houben, L.; Seifert, G. Nanoseashells and nanooctahedra of $\mathrm{MoS}_{2}$ : Routes to inorganic fullerenes. Chem. Mater. 2009, 21, 5627-5636.

19. Albu-Yaron, A.; Levy, M.; Tenne, R.; Popovitz-Biro, R.; Weidenbach, M.; Bar-Sadan, M.; Houben, L.; Enyashin, A.N.; Seifert, G.; Feuermann, D.; et al. $\mathrm{MoS}_{2}$ hybrid nanostructures: from octahedral to quasi-spherical shells within individual nanoparticles. Angew. Chem. Int. Ed. 2011, 123, 1850-1854.

20. Alexandrou, I.; Sano, N.; Burrows, A.; Meyer, R.R.; Wang, H.; Kirkland, A.I.; Kiely, C.J.; Amaratunga, G.A.J. Structural investigation of $\mathrm{MoS}_{2}$ core-shell nanoparticles formed by an arc discharge in water. Nanotechnology 2003, 14, 913-917.

21. Gordon, J.M.; Katz, E.A.; Feuermann, D.; Albu-Yaron, A.; Levy, M.; Tenne, R. Singular MoS2, $\mathrm{SiO}_{2}$ and $\mathrm{Si}$ nanostructures - synthesis by solar ablation. J. Mater. Chem. 2003, 18, 458-462.

22. Journet, C.; Maser, W.K.; Bernier, P.; Loiseau, A.; de-la Chapelle, M.L.; Lefrant, S.; Deniard, P.; Lee, R.; Fischer, J.E. Large-scale production of single-walled carbon nanotubes by the electric-arc technique. Nature 1997, 388, 756-758.

23. Qin, L.-C.; Zhao, X.; Hirahara, K.; Miyamoto, Y.; Ando, Y.; Iijima, S. Materials science: The smallest carbon nanotube. Nature 2000, 408, 50.

24. Wang, N.; Tang, Z.K.; Li, G.D.; Chen, J.S. Materials science: Single-walled 4 A carbon nanotube arrays. Nature 2000, 408, 50-51.

25. Hernandez, E.; Goze, C.; Bernier, P.; Rubio, A. Elastic properties of $\mathrm{C}$ and $\mathrm{B}_{x} \mathrm{C}_{y} \mathrm{~N}_{z}$ composite nanotubes. Phys. Rev. Lett. 1998, 80, 4502-4505.

26. Lorenz, T.; Teich, D.; Joswig, J.-O.; Seifert, G. Theoretical study of the mechanical behavior of individual $\mathrm{TiS}_{2}$ and $\mathrm{MoS}_{2}$ nanotubes. J. Phys. Chem. C 2012, 116, 11714-11721. 
27. Koshland, D.E. Stereochemistry and the mechanism of enzymatic reactions. Biolog. Rev. 1953, 28, 416-436.

28. Herrmann, I.; Brüser, V.; Fiechter, S.; Kersten, H.; Bogdanoff, P. Electrocatalysts for oxygen reduction prepared by plasma treatment of carbon-supported cobalt tetramethoxyphenylporphyrin. J. Electrochem. Soc. 2005, 152, A2179-A2185.

29. Meichsner, J.; Schmidt, M.; Schneider, R.; Wagner, H.E. Nonthermal Plasma Chemistry and Physics; CRC Press: Boca Raton, FL, USA, 2013; Section 3.8.7, pp. 120-121.

30. Savastenko, N.A.; Müller, S.; Anklam, K.; Brüser, M.; Quade, A.; Walter, C.; Brüser, V. Effect of plasma treatment on the properties of Fe-based electrocatalysts. Surf. Coat. Technol. 2011, 205, 439-442.

31. Kortshagen, U. Nonthermal plasma synthesis of semiconductor nanocrystals. J. Phys. D Appl. Phys. 2009, 42, 113001. 


\title{
IF-WS 2 /Nanostructured Carbon Hybrids Generation and Their Characterization
}

\section{Claudia C. Luhrs, Michael Moberg, Ashley Maxson, Luke Brewer and Sarath Menon}

\begin{abstract}
With the aim to develop a new generation of materials that combine either the known energy absorbing properties of carbon nanofibers (CNF), or the carbon-carbon bond strength of graphene sheets $(\mathrm{G})$, with the shock resistance properties reported for Inorganic Fullerene type $\mathrm{WS}_{2}$ structures (IF-WS 2 ), hybrid CNF/IF-WS 2 and G/IF-WS 2 were generated, characterized and tested. Experimentation revealed that in situ growth of carbon nanostructures with inorganic fullerene tungsten disulfide particulates had to be performed from particular precursors and fabrication conditions to avoid undesirable byproducts that hinder fiber growth or deter graphene generation. The novel protocols that allowed us to integrate the IF-WS 2 with the carbon nanostructures, producing dispersions at the nanoscale, are reported. Resulting hybrid CNF/IF-WS 2 and G/IF-WS products were analyzed by X-ray Diffraction (XRD), Scanning Electron Microscope (SEM) and TEM (Transmission Electron Microscopy). The thermal stability of samples in air was evaluated by Thermogravimetric Analysis (TGA). CNF/IF-WS 2 and G/IF-WS 2 hybrids were introduced into epoxy matrices, and the mechanical properties of the resulting composites were analyzed using nanoindentation. Epoxy composite samples showed drastic improvements in the Young's modulus and hardness values by the use of only $1 \%$ hybrid weight loadings. The carbon nanofiber inclusions seem to have a much greater impact on the mechanical properties of the composite than the graphene based counterparts.
\end{abstract}

Reprinted from Inorganics. Cite as: Luhrs, C.C.; Moberg, M.; Maxson, A.; Brewer, L.; Menon, S. IF-WS 2 /Nanostructured Carbon Hybrids Generation and Their Characterization Inorganics 2014, 2 , $211-232$.

\section{Introduction}

Tungsten disulfide inorganic fullerene-like particulates (IF-WS 2 ), a dichalcogenide with distinct physical and chemical properties [1,2] that presents a hollow cage structure with potential uses as lubricant, component in batteries, supercapacitors or catalyst, among others [3-9], was introduced a few years ago as a material with shock resistance properties [10-13]. The shock absorbing ability of IF-WS 2 particulates allows them to endure pressures up to $25 \mathrm{GPa}$, with concomitant temperatures of up to $1000{ }^{\circ} \mathrm{C}$, without structural degradation or phase change [12], a characteristic that opens an exciting window of possibilities for protective systems applications. Recent reports have further explored the features of such $\mathrm{WS}_{2}$ nanostructures and some studies of their inclusion in polymeric matrices, along with the composites mechanical properties, have been published [14-24]. Despite those outstanding characteristics, the $\mathrm{WS}_{2}$ density might be considered a drawback if it is to be used as component in protective gear that should be lightweight, able to withstand high temperatures and be flame resistant. A possible compromise between all those requirements might be made by joining the sought-after shock absorbing attribute of IF-WS 2 with lighter materials. 
Due to their strength and light weight, tridimensional carbon assemblies and porous carbon structures such as nanotubes, foams or intertwined nanofibers [25-37], along with two-dimensional structures, such as Graphene [38-40], have been the focus of attention for energy absorbing applications. However, despite the advances in the field, the performance of personal protective equipment or sporting gear composed of carbon nanostructures can still be improved by the use of materials that could divert, distribute or dissipate the energy of impacts and the shock waves associated with them, in a more efficient manner.

Given the shock resistance characteristics of IF-WS 2 and the energy absorption of carbon nanostructures mentioned above, the combination of those two types of materials seems as a natural next step in the design of protective systems. Recent work that has successfully explored the possibility of merging the carbon component with IF-WS 2 using polymeric matrices $[16,18,41-45]$. However, the energy dissipation characteristics of those products could be attributed, to an extent, to the role of the viscoelastic polymeric matrices. Conversely, new reports in the generation of carbon fiber tridimensional structures have proven that CNF can be grown to form a macroscopic foam with viscoelastic properties without the need of a polymeric component [46]. Adding the known shock absorbing characteristics of IF-WS 2 to such type of carbon structures will be highly desirable and is the focus of this investigation.

The development of a protocol to combine the IF-WS 2 characteristics with those of the carbon structures in the absence of a polymer, to generate a random distribution of those two phases at the nanoscale ( $c a$. a hybrid made of solely inorganic components, thus, avoiding some of the polymer drawbacks such as aging but gaining in terms of lightweight and thermal stability), remains as one of the major challenges.

Experimentation by our group has shown that adding IF-WS 2 to an already existing 3D carbon structure, even when using solvents to achieve the mixture, renders an inhomogeneous solid. The present work aims to produce a well-dispersed hybrid system composed of a carbon solid (Carbon Nanofiber or Graphene) and low loading levels of IF-WS 2 . We found that to produce a well dispersed $3 \mathrm{D}$ structure of carbon nanofibers and IF-WS 2 it is necessary to employ the two stage in situ protocol described in the next section. In contrast, to produce a hybrid of Graphene (2D layered structure) with IF-WS 2 , the use of the in situ protocol did not show significant improvement in the phase distribution when compared to physically mixing the components.

As an extension of the primary goals, we studied the mechanical properties of epoxy composites based on hybrid CNF/IF-WS 2 and G/IF-WS 2 made by in situ routes, and contrast the latter to those created from physical mixtures of the components.

\section{Results and Discussion}

With the goal of generating hybrid Carbon nanostructure/IF-WS 2 we conducted the steps summarized in the experimental section and produced samples whose qualities are described below. We have divided the results and discussion segment into three parts; the first one presenting the microstructural characteristics and stability of samples based on CNF/IF-WS 2 , the second one including the study of Graphene/IF-WS 2 and the third showing the mechanical properties of both $\mathrm{CNF} / \mathrm{IF}-\mathrm{WS}_{2}$ and Graphene/IF-WS 2 epoxy composites. The samples made in the absence of polymer 
were denominated hybrids and the ones with nanostructures embedded in epoxy are referred as composites as a way to distinguish them.

\subsection{Carbon Nanofiber/IF-WS 2 Hybrids}

The combination of existing 3D CNF structures with IF-WS 2 in the absence of a polymeric matrix, as mentioned in the introduction section, results in inhomogeneous solids. For example, the addition of IF-WS 2 to already existing CNF generates samples where diffusion paths dominate the final structure: the surface of the carbon fibers tends to contain larger amounts of the IF-WS 2 phase than sections of the sample not exposed to the surface. The use of solvents and sonication to disperse the IF or its precursors, is not enough to produce a homogeneous product. A second example; The addition of metal catalyst particles (to promote the carbon fiber growth) to existing IF-WS $\mathrm{W}_{2}$ followed by thermal treatments to proceed with the carbon nanofibers growth in the presence of a carbon source, produces a thin layer of metal sulfides in the surface of the metal particles. This phenomenon is observed also in the absence of carbon sources: at moderate temperatures (below $350{ }^{\circ} \mathrm{C}$ according to our findings) the metal reacts with the tungsten sulfide producing thin films of secondary phases in the metal particle surface, which in practice poison the catalyst, capping it and hindering the growth of the carbon fibers. Hence, this method also fails to produce the expected product. Variations in the experimental parameters, such as gas flows, placement of precursors, size and nature of metal catalyst, amid others, generates, in the best case scenario, amorphous carbonaceous byproducts of different sizes and morphologies, far from the desired fiber or porous structures [47].

Diverse precursor options could be used to generate IF-WS 2 structures; $\left(\mathrm{NH}_{4}\right)_{2} \mathrm{WS}_{4}$ thermal decomposition and $\mathrm{WO}_{3}$ reaction with sulfur-containing compounds are among the most common routes employed [1,48-56]. The generation of IF-WS 2 carried out in our laboratories using such precursors resulted in samples with different levels of long range order and particle size, as can be inferred from its XRD pattern and SEM analysis. Figure 1 presents the $\mathrm{WS}_{2}$ patterns obtained when decomposing $\left(\mathrm{NH}_{4}\right)_{2} \mathrm{WS}_{4}$ and from the reaction of commercial $\mathrm{WO}_{3}$ nanoparticles with $\mathrm{H}_{2} \mathrm{~S}$ atmospheres. It is worth noting that the temperatures needed for such reactions to occur differ, the former was performed at moderate temperatures $\left(\mathrm{ca} .500{ }^{\circ} \mathrm{C}\right)$ while the latter required higher temperatures to be carried out to completion $\left(c a .800^{\circ} \mathrm{C}\right)$. The diffraction peaks for IF-WS 2 generated from $\mathrm{WO}_{3}$ are narrower and the reflections $(001)$ with $1=2 n$ more evident and well defined than the ones obtained from $\left(\mathrm{NH}_{4}\right)_{2} \mathrm{WS}_{4}$. SEM observation of both products confirmed the presence of the hollow cage structures, the so-called 3R phase. Moreover, the former tetrathiotungstate precursor reacts with metal particles when it decomposes, which makes $\mathrm{WO}_{3}$ the best choice to be combined with metal catalyst for fiber growth.

The two stage in situ synthesis of homogeneous CNF/IF-WS 2 required diverse atmospheres and temperature steps to be accomplished successfully. The first stage had the objective to grow CNF from a metal catalyst intermixed with $\mathrm{WO}_{3}$ and the second stage consisted on sulfurizing the mixture of $\mathrm{CNF} / \mathrm{WO}_{3}$ to transform the tungsten oxide into IF-WS 2 .

The dispersion of the metal particle (nickel in this case) with the $\mathrm{WO}_{3}$ nanoparticles was performed using a solvent and sonication, followed by evaporating the mixture until dry since it rendered a more homogenous precursor than simply grinding the solids. A first temperature step was 
carried out at $350{ }^{\circ} \mathrm{C}$ in reducing atmospheres as a precautionary measure to assure that the metal catalyst surface was free of oxides. A second step at the same temperature including ethylene (as carbon source) and small amounts of oxygen diluted in inert gas (as reaction initiator and radical originator) was performed to cover the metal particle with an initial thin layer of carbon (which prevents the metal gross agglomeration and sintering at higher temperatures). The growth step was made at $550{ }^{\circ} \mathrm{C}$, following protocols previously developed [57]. A final temperature stage at $900{ }^{\circ} \mathrm{C}$ in $\mathrm{H}_{2} \mathrm{~S}$ containing environment was included to transform the $\mathrm{WO}_{3}$, now dispersed in between carbon nanofibers, into IF-WS 2 . The sample was then allowed to cool to room temperature using an inert atmosphere.

Figure 1. XRD patterns of IF-WS 2 particles generated from different precursors. IF-WS 2 generated from (a) commercial $\mathrm{WO}_{3}$ particles; (b) from ammonium tetrathiotungstate. All peaks were identified as IF-WS 2 [12].

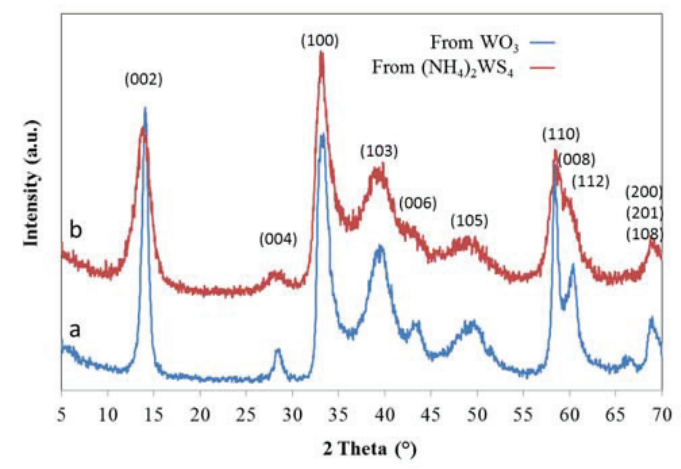

The electron micrographs of the nickel nanoparticles employed, the carbon nanotube intertwined nanofibers generated and the hybrid CNF/IF-WS 2 are presented in Figure 2. The ratios of catalyst to $\mathrm{WO}_{3}$ used were designed to have nominal loading values of $0.5,1.5$, and $5 \%$ of IF-WS 2 with respect to the total weight of the CNF. The distribution of the IF particles was studied using SEM in Secondary Electron (SE) and Backscattered Electron (BSE) modes along with Energy Dispersive Spectroscopy (EDS) mapping and can be described as discrete pockets of cage like particulates of approximately 1-2 microns dispersed into the CNF threads (Figure 2c). This finding is consistent across different sample locations.

The catalyst used for the carbon nanofibers was not removed and represents close to $3 \%$ of the total weight of the sample. Its effects on the properties of the epoxy composites are discussed in Section 2.3.

The last stage of the in situ protocol, the sulfurization, transforms not only the $\mathrm{WO}_{3}$ into $\mathrm{WS}_{2}$ but the nickel catalyst into nickel sulfide. Indeed, the hybrid CNF/IF-WS 2 samples are composed by the solids mentioned in Table 1 (experimental section), where nominal values and elemental analysis by ICP methods are contrasted. The inductive plasma emission spectroscopic data shows that the final composition attained is within $0.2 \%$ of the targeted values. 
As a means to understand the characteristics of the samples and the different phases formed at the diverse synthesis steps, some of the runs where halted before completion and the products at that point, were analyzed by diverse techniques. As part of those trials, experiments of fiber growth in the absence of $\mathrm{WO}_{3}$ with and without sulfurization treatment were performed. The X-ray diffraction analysis of the latter, containing only CNF samples, is presented in Figure 3. The main reflection, close to $26^{\circ}$, corresponds to the (002) peak of graphite, characteristic of many carbon products that include a crystalline component with various degrees of basal plane alignment [58]. In the present case the peak is associated to the CNF presence. The diffraction pattern of samples after the carbon fiber growth step but before sulfurization (red line in Figure 3), show only the peaks of graphite and a couple of reflections, close to 45 and $52^{\circ}$, that correspond to the nickel particles used as catalyst. The phases encountered after sulfurization (blue line) corroborate the presence of two extra crystalline structures, nickel sulfide: $\mathrm{Ni}_{3} \mathrm{~S}_{2}[59,60]$ and $\mathrm{Ni}_{17} \mathrm{~S}_{18}[61]$.

Figure 2. Microstructural Analysis of CNF and $\mathrm{WS}_{2}$ precursors and CNF/IF-WS 2 hybrid. Scanning Electron Microscopy secondary electron images of (a) starting nickel nanoparticles; (b) carbon nanofibers grown from Ni catalyst; (c) nanoparticle dispersion and cluster of $\mathrm{WS}_{2}$ nanoparticles within carbon nanofibers (CNF/IF-WS 2 hybrid).
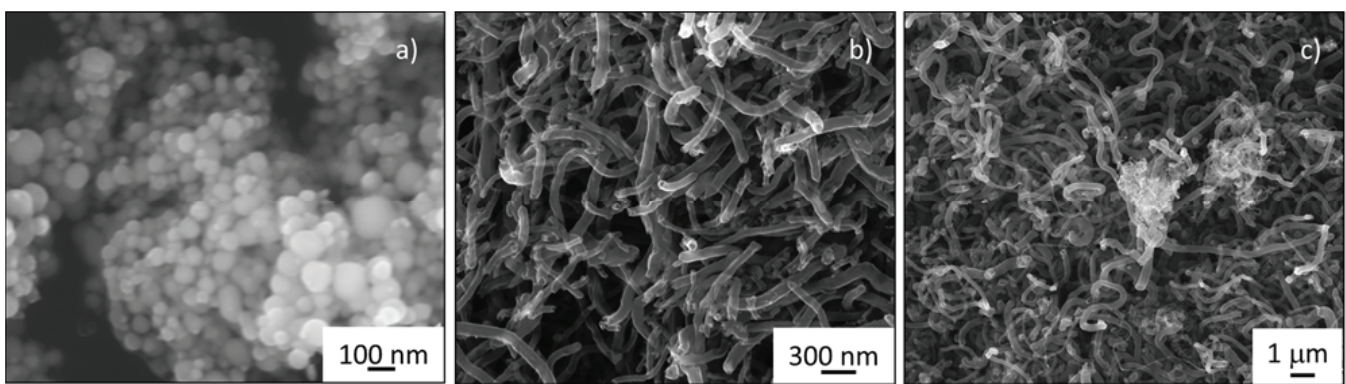

Figure 3. X-ray diffraction pattern of carbon nanofibers. (a) Typical peaks of graphite and nickel metal are identified in samples as prepared; (b) XRD pattern after sulfurization step shows graphite and nickel sulfide reflections.

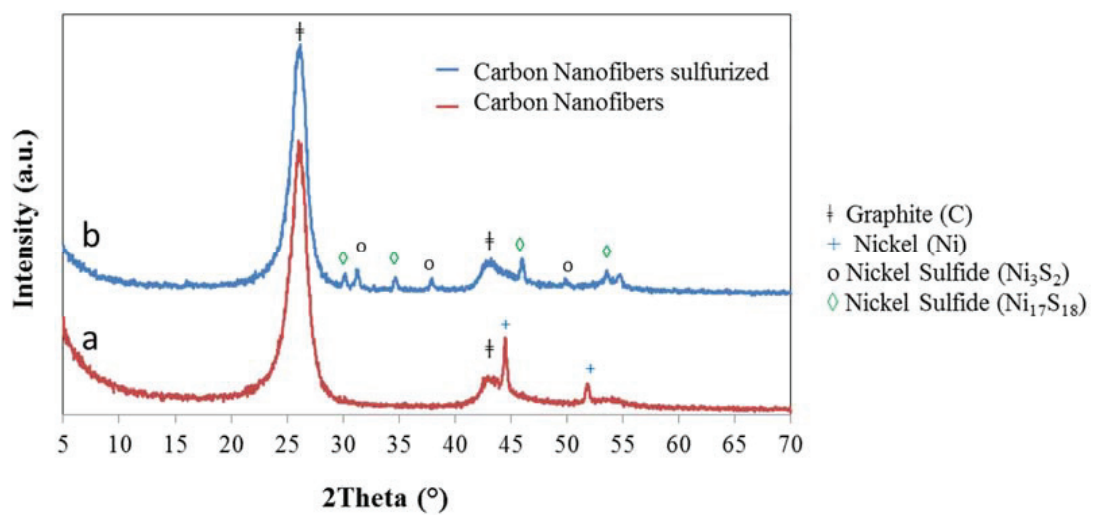


The XRD patterns of CNF/IF-WS 2 hybrids at the three different levels of loadings prepared are shown in Figure 4. The same phases recognized in Figure 3 after sulfurization; graphitic peaks, $\mathrm{Ni}_{3} \mathrm{~S}_{2}$ and $\mathrm{Ni}_{17} \mathrm{~S}_{18}$, along with a comparatively weak peak corresponding to IF-WS2, can be identified.

Figure 4. X-ray diffraction patterns for hybrid CNF/IF-WS 2 samples. The main difference between XRD patterns in samples at diverse loadings is the relative intensity for the nickel sulfide $v s$. graphitic peaks.

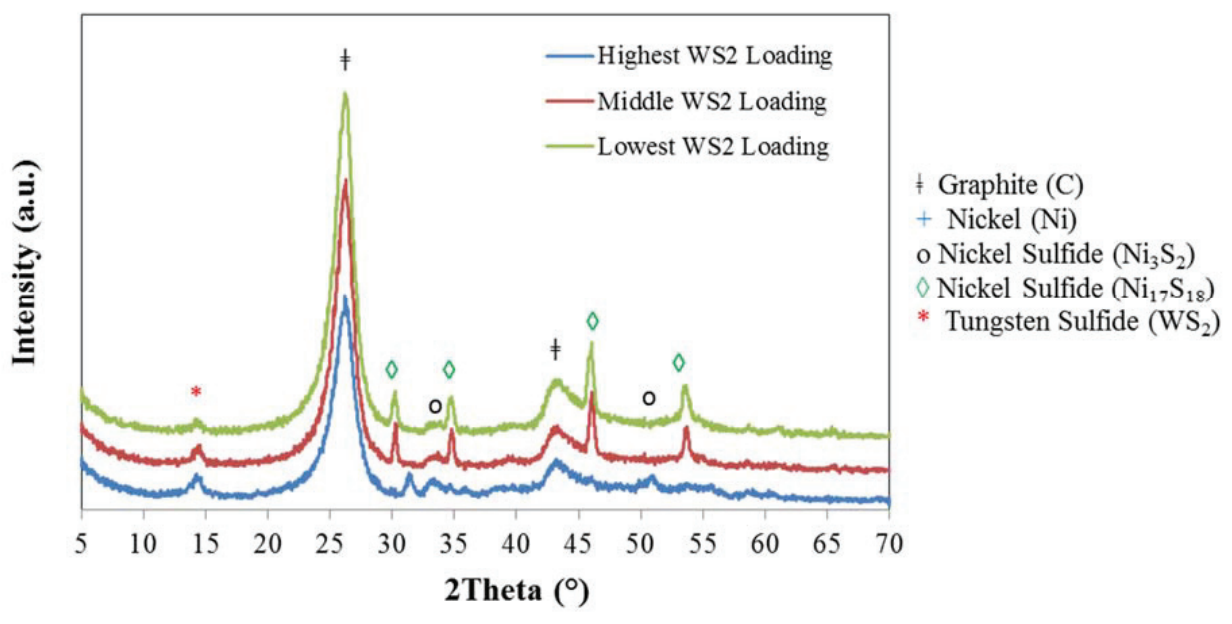

SEM in BSE mode studies along elemental mapping by EDX confirmed the presence of IF-WS 2 as highly dispersed phase. Figure 5 shows the metal distribution as bright spots (top left), including both nickel and tungsten phases. The IF-WS 2 distribution can be inferred as the positions where W elemental mapping is found (top right). The W mapping shows the presence of mainly small (nm) particulates homogeneously distributed in the sample. In contrast, the Ni particles used as catalyst, now converted into sulfides, appear both as nanometer particulates and micron size agglomerates; with dimensions that could be correlated with the different fiber diameters (bottom right). The fact that the nickel elemental distribution shows micron size clusters is a sign that some agglomeration of some of the initial particles might have occurred during the fiber growth, since the original catalyst had a size distribution in the nm range, as shown in Figure 2a. The sulfur map (bottom left) encompasses both, the IF-WS 2 and Ni sulfide components.

The samples' thermal analysis under oxygen containing atmospheres show that all specimens; $\mathrm{CNF}$ (catalyst included), $\mathrm{CNF}$ sulfurized and hybrid $\mathrm{CNF} / \mathrm{IF}-\mathrm{WS}_{2}$ are stable at least up to a temperature of $500{ }^{\circ} \mathrm{C}$ (Figure 6). At higher temperatures the carbonaceous component burns off to produce $\mathrm{CO}_{2}$, the tungsten sulfide reverts to its oxidized form and the metal catalyst oxidizes. Experiments conducted in inert atmosphere showed that hybrid CNF/IF-WS 2 samples do not present weight changes in the window of conditions used (RT to $950{ }^{\circ} \mathrm{C}$ ) and can be considered thermally stable. Indeed, the hybrid samples stability observed surpasses the ones observed for polymer products without $\mathrm{WS}_{2}$, which in either atmosphere start decomposing at much lower temperatures, and are in agreement with stability observed in air for IF-WS 2 based composites $[19,20,62]$. The 
reported thermal stability of $\mathrm{WS}_{2}$ fullerene-like particles alone under oxidizing atmospheres indicates that, depending on particle size, oxidation might begin close to $290{ }^{\circ} \mathrm{C}(100 \mathrm{~nm})$ or delay up to close to $440{ }^{\circ} \mathrm{C}(3 \mathrm{~m})$, while exposed to inert gases the phase is stable up to $1200{ }^{\circ} \mathrm{C}[63,64]$. In our case, the use of $\mathrm{CNF}$ without polymeric component seems to increase the thermal stability of the hybrid, given that the average IF-WS 2 particle size produced was in the nanometer scale (expected to oxidize close to $300{ }^{\circ} \mathrm{C}$ ) and changes in weight were not evident until above $\sim 500{ }^{\circ} \mathrm{C}$. The thermal stability observed for IF-WS 2 might be related to the particulates being embedded in the CNF intertwined fibers, which might delay their reaction. Further studies to fully explain these phenomena are currently under way.

Figure 5. Phase distribution studied by SEM/EDX analysis. Top left: Backscattered electron image showing the carbon fibers in grey and the metal containing phases as bright spots in the sample CNF/IF-WS 2 with the lowest IF-WS 2 loading. Elemental mapping by EDX analysis confirmed the presence of IF-WS 2 mainly as highly dispersed phase (top right) with small (nm) particle size. The elemental Ni map shows the presence of nm and micron size particulates (bottom right). The sulfur map (bottom left) includes both, the IF-WS 2 and Ni sulfide components.
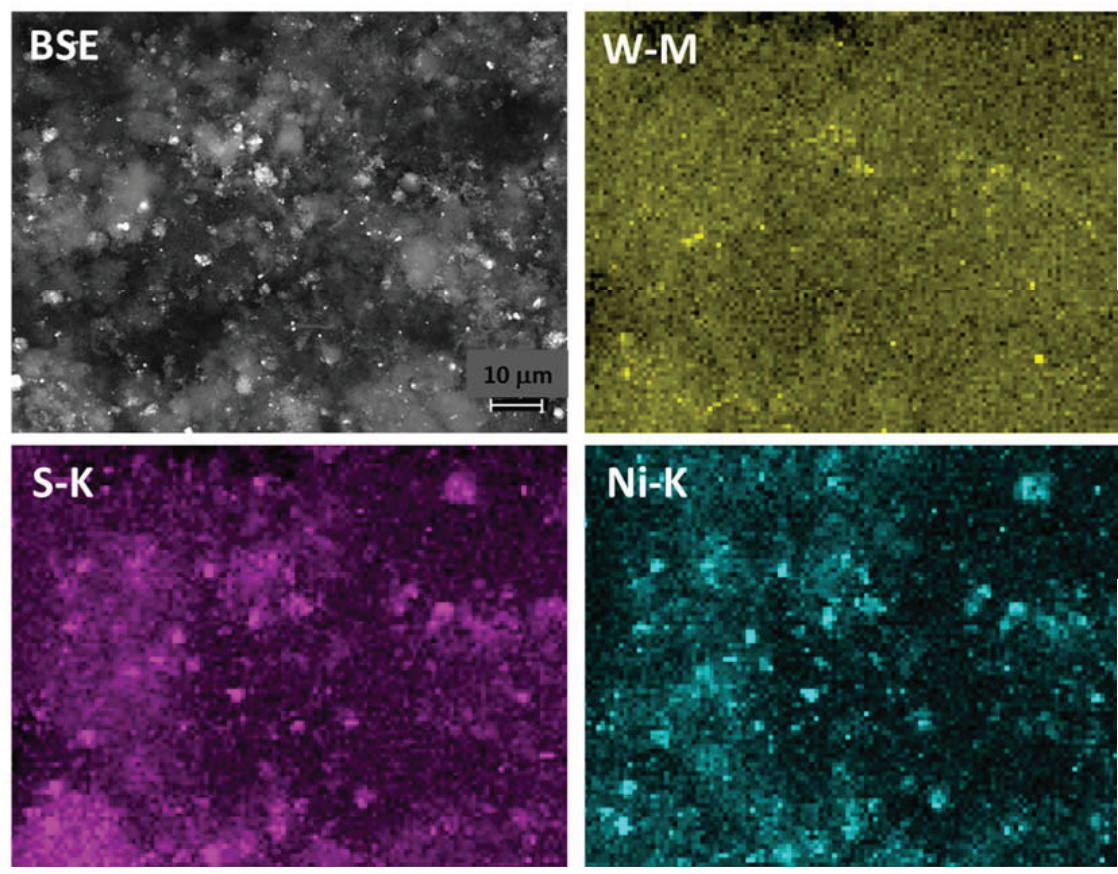
Figure 6. Thermogravimetric Analysis. Temperature programmed oxidation (burn off process) for (a) carbon nanofibers-including nickel catalyst; (b) sulfurized carbon nanofibers -including nickel sulfide and (c) $\mathrm{CNF} / \mathrm{IF}-\mathrm{WS}_{2}$ hybrid with $3 \%$ tungsten sulfide loading. All of the samples are stable in oxygen containing environment up to $500{ }^{\circ} \mathrm{C}$.

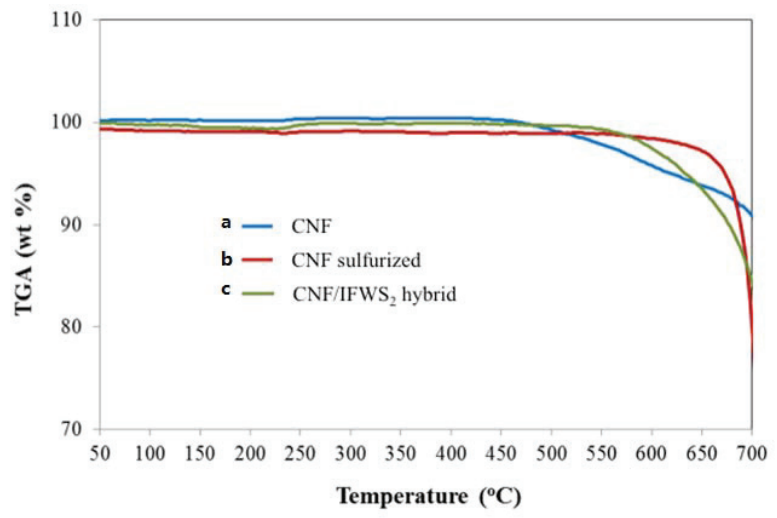

\subsection{IF-WS $/$ Graphene Hybrids}

The complications introduced by the use of metal catalyst for nanofibers growth were not encountered during the synthesis of Graphene/IF-WS2 hybrids, since no extra metal components were needed to generate them. Only small amounts of urea were used as an expansion agent. The production of graphene was accomplished by the reduction-expansion of graphite oxide (GO), which was generated from graphite flakes by the process described in the experimental section. The GO mediated process generates disordered graphene; where graphene sheets tend to entangle with each other but remain separated enough to maintain a relative high surface area $\left(c a .600 \mathrm{~m}^{2} / \mathrm{g}\right.$ as per BET analysis). It is worth noting that the oxygen content in the synthesis environment when using GO as a carbon precursor is sufficient to oxidize some of the IF-WS 2 if the latter is used since the beginning of the protocol, forcing the use of $\mathrm{WO}_{3}$ as the tungsten source. Thus, $\mathrm{WO}_{3}$ nanoparticles were subject to $\mathrm{H}_{2} \mathrm{~S}$ treatments to sulfurize them after its mixtures with graphene were obtained.

The two variations on the synthesis conditions consisted of: (i) dispersing the $\mathrm{WO}_{3}$ nanoparticles with $\mathrm{GO}$ and then performing the exfoliation at high temperature (to generate Graphene/ $\mathrm{WO}_{3}$ ) followed by sulfurization (to obtain Graphene/IF-WS 2 ) or (ii) perform the GO exfoliation first and then directly mixing the resulting graphene with $\mathrm{WO}_{3}$ and then sulfurizing the product (rendered Graphene/IF-WS 2 ). The microstructures created are presented in Figure 7.

The first synthetic approach, $\mathrm{GO}$ plus $\mathrm{WO}_{3}$ exfoliation followed by sulfurization, resulted in hybrid Graphene/IF-WS 2 where the IF structures were located on the surface of disordered graphene sheets (marked by arrows, Figure 7a). Due to the thermal exfoliation and urea reduction process, when volatile groups leave the graphite oxide structure at temperatures close to $200{ }^{\circ} \mathrm{C}$, they carry along the $\mathrm{WO}_{3}$, which ends in top of the layers, where they remain through the sulfurization step that converts them into IF-WS 2 cage structures. The exfoliation process separates the graphene sheets but also promotes the separation of IF particles, which were found in all cases as individual particles. 
The position of the IF particulates following the second synthetic approach, is quite distinct. In this protocol, GO is first thermally exfoliated and reduced and the subsequent graphene is mixed with $\mathrm{WO}_{3}$ (with the aid of solvents and sonication, followed by solvent evaporation until dry) and the mixture is then sulfurized. The IF particulates are found between the graphene layers, intimately embedded into the sheets structure (Figure 7b). Small (few nm diameter) IF particulate agglomerates, marked in the image with a blue arrow, were observed along individual particles, indicated by red arrows. The IF-WS 2 observed by Transmission Electron Microscopy presents the characteristic hollow cage, partially faceted structure and an interlayer spacing of $0.62 \mathrm{~nm}$.

For comparison, samples in which Graphene and IF-WS 2 were formed as individual phases and then added as a physical mixture using solvents and sonication (not grown in situ), present similar structures to the ones in Figure 7b, demonstrating that the protocols developed to introduce IF-WS2 into carbon structures are required only when tridimensional architectures of the carbonaceous component are needed but not if a layered, two dimensional structure is used.

Figure 7. Microstructural Analysis of Graphene/IF-WS 2 hybrids and IF-WS 2 . Scanning Electron Microscopy images of (a) Graphene/IF-WS 2 hybrid generated from GO and $\mathrm{WO}_{3}$; (b) Graphene/IF-WS 2 hybrid made directly from Graphene and $\mathrm{WO}_{3}$; and (c) Transmission electron micrograph showing the characteristic hollow core and interlayer spacing for IF-WS 2 particles.
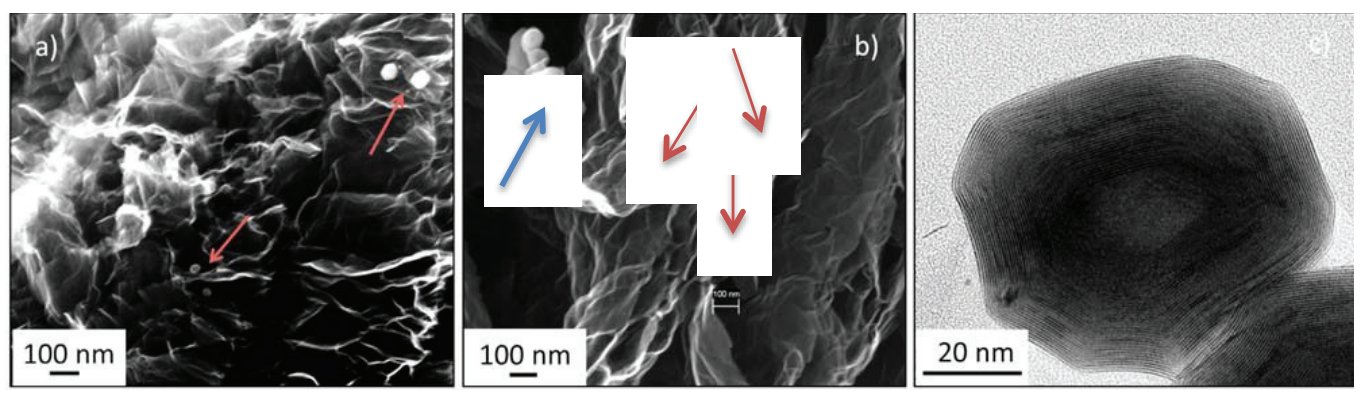

\subsection{Epoxy Composites}

The use of epoxy as polymeric matrix was intended (independent of its properties as a composite for use in protection systems) to determine the mechanical properties observed when using carbon nanomaterials with 3D vs. 2D structures (CNF vs. Graphene) that included low loadings of IF-WS2. Other instances of $\mathrm{WS}_{2}$ nanostructures embedded in epoxy can be found in [14,23], where $\mathrm{WS}_{2}$ nanotubes were used as filler instead of the IF particulates used in this study. To the best of our knowledge, the only existing references including IF-WS 2 particles in epoxy systems do not involve a carbonaceous component [21-24].

Experimental testing in our laboratory and previous epoxy resin composite research data $[47,65]$ were used to select $1 \%$ as targeted loading of filler material into epoxy matrix. Bare epoxy resin, produced in identical conditions to the ones containing diverse fillers, was used as reference. Table 1 presents the values for the different filler components used to prepare the composites, all 
cases include only $1 \%$ of total filler and $99 \%$ of epoxy, by weight. On the table, the first column contains the nominal values of IF-WS 2 component targeted during synthesis, the rest of the columns are the values encountered when analyzing the filler samples by ICP Emission Spectroscopy.

Two types of nanoindentation measurements showed clear increases in the Young's modulus of the epoxy-CNF composites. As described in the experimental section, dynamic mechanical analysis was performed on epoxy-CNF composites to ascertain the degree of viscoelastic deformation of these materials at room temperature. At loading frequencies of both $1 \mathrm{~Hz}$ and $45 \mathrm{~Hz}$, the elastic modulus response was dominated by the storage (elastic) modulus with a very minor contribution, less than $5 \%$, from the loss (viscous) modulus. As such, quasi-static indentation was deemed appropriate for these materials. We used quasi-static nanoindention to measure the Young's modulus and hardness for each of the composites using the conditions listed in Table 2. The inclusion of carbon nanofibers into the epoxy matrix increases the epoxy modulus by $29 \%$. CNF sulfurized, with no IF-WS 2 but subject to sulfurization process with $\mathrm{H}_{2} \mathrm{~S}$, improves it nearly by $52 \%$ (Figure 8a). A possible explanation to the higher modulus values for the later might be related to the presence of sulfur and its effects on the epoxy-CNF interface adhesion. The three hybrid samples tested, CNF containing IF-WS 2 particulates with $0.5,1.5$, and $3 \%$, show much higher modulus than the rest, with values that almost double the one for bare epoxy (for hybrid CNF/IF-WS $2.5 \%$ ). While we observed a clear variation in sample performance for hybrids containing CNF and diverse IF-WS 2 contents, the data does not show a clear correlation with the actual amount of IF added.

For all samples that included fillers we observed a more dramatic change in hardness than for the elastic modulus; the initial addition of carbon nanofibers had a large effect and increased the hardness by more than $114 \%$ (Figure $8 \mathrm{~b}$ ). The addition of sulfurized fibers further raised the hardness by another $38 \%$. The sulfurization of the fibers appears to improve the ability of the fibers to bond with the epoxy matrix, further improving the interfacial strength. The increase in hardness could also be partially due to the inclusion of nickel sulfide particles. As shown in Figures 3 and 4, after the sulfurization step is carried out all nickel used as catalyst for the CNF growth transforms into $\mathrm{Ni}_{3} \mathrm{~S}_{2}$ or $\mathrm{Ni}_{17} \mathrm{~S}_{18}$. While these particles contribute to an increase in performance in this case, it is possible that other effects could appear under different situations.

Nickel sulfide contamination is an ongoing concern for the tempered glass industry. High temperature structures of nickel sulfide can develop in the glass manufacturing process and become included in tempered glass. This inclusion will result in a stress concentration and if the particle is of a certain size, the pane of glass will shatter under loading far below what is expected of the material [66]. Depending on the future inclusions of these materials into other matrixes, the possible impact of sulfide particles as stress concentrators should be considered and further studied. In the case of polymeric matrices, like the ones studied herein, no failure modes related to the existence of nickel sulfide are expected, since the working and processing temperatures do not reach those where the nickel sulfide high temperature phase appears $\left(715^{\circ} \mathrm{C}\right)$. In the case of hybrids in the absence of polymers, the porous carbon fiber structure is presumed to accommodate the volume expansion of the phase change and does not constitute a concern.

All the hybrid CNF/IF-WS 2 samples showed a significant increment in hardness values, being $\mathrm{CNF} / \mathrm{IF}-\mathrm{WS}_{2} 0.5 \%$ the most remarkable, with a $247 \%$ improvement over the pure epoxy hardness. 
Figure 8. Mechanical properties of epoxy composites. All composites contained $99 \%$ epoxy and 1\% loading of filler nanostructures: CNF, sulfurized CNF or hybrid $\mathrm{CNF} / \mathrm{IF}-\mathrm{WS}_{2}$. For the later, the hybrids contained mostly carbon fibers, with only 0.5 , 1.5 , and $3 \%$ of IF-WS 2 . (a) Modulus data and (b) Hardness values.

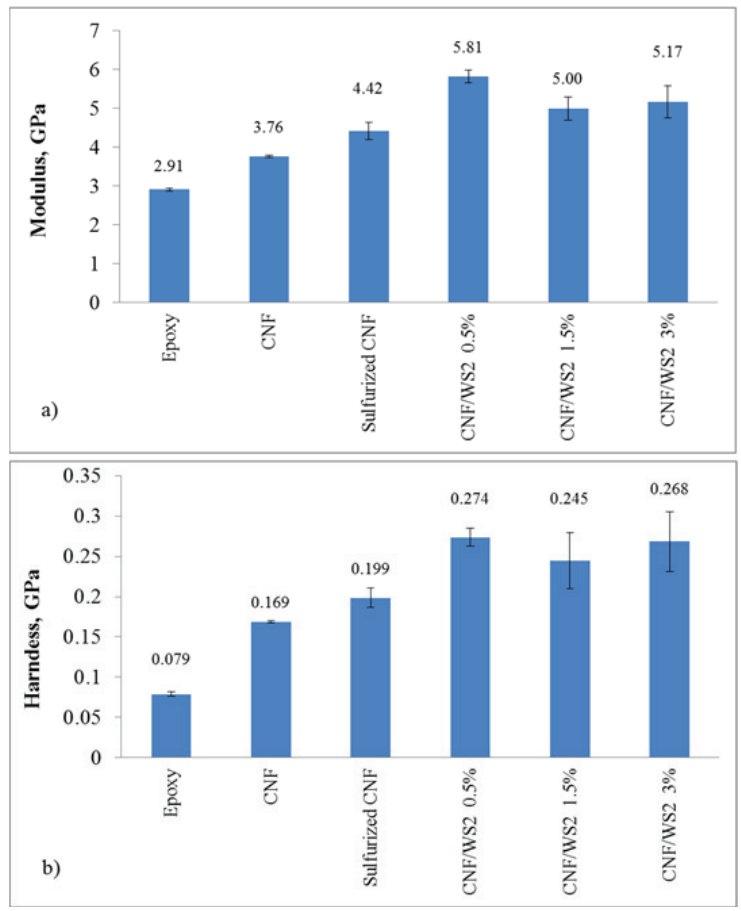

In order to fully understand the CNF/IF-WS2 epoxy composite mechanical properties observed, further study of their interfaces by IR or other spectroscopic techniques is recommended. Such data will help characterize the changes introduced by the inorganic components in the oxirane ring bands, the overall epoxy resin structure and its degree of polymerization.

The inclusion of two-dimensional graphene into the epoxy matrix in equal loadings than the ones described above, $1 \mathrm{wt} \%$ filler in epoxy, by itself or as Graphene/IF- $\mathrm{WS}_{2}$, resulted in increased values of modulus and hardness (Figure 9). However, the improvement when compared to the 3D nanofiber structures is modest; the hybrid Graphene/IF-WS 2 with $0.5 \%$ of IF-WS 2 showed a $9 \%$ modulus increment over bare epoxy and a $47 \%$ increase in hardness. Histograms for the composites that contain CNF have been highlighted in blue and the ones containing graphene in red. From the Figure is clear that the samples based in $3 \mathrm{D}$ CNF architectures containing IF-WS 2 present the highest values. During the composite fabrication, the graphene based samples seemed to be more difficult to disperse into the epoxy matrices than the CNF ones and inconsistencies in the sample distribution into the epoxy puck might have a detrimental effect on the mechanical properties values. Moreover, the fact that the graphene sheets were distributed randomly in the sample and not oriented in the (002) direction, where the strong covalent bonds are located, might be another point to be considered to explain the observed mechanical behavior. 
As mentioned in Section 2.2, the use of Graphene/IF-WS 2 prepared in situ vs. the components physical mixtures do not seem to present an advantage in terms of phases distribution or their microstructural characteristics. In terms of mechanical properties, the in situ route and the physical mixtures of Graphene/IF-WS 2 are comparable; the values for modulus are almost the same and the hardness is higher for the former. This result might be related to the fact that IF particulates might easily disperse in between the graphene sheets without the need of a multistep process. It is worth noting that in the sample created in situ, the graphene structure has been exposed to $\mathrm{H}_{2} \mathrm{~S}$ atmospheres, while in the physical mixture the graphene was pristine and never in contact with sulfurizing environments. Given that the improvement in modulus and hardness over neat epoxy observed in those samples was minimal, the present study did not further investigate the interfacial effects created by diverse functionalities in the graphene surface.

Figure 9. Mechanical properties of epoxy composites. with 1\% loading of $\mathrm{CNF}$, sulfurized CNF, Graphene, IF-WS 2 , CNT/IF-WS 2 hybrids or Graphene/IF-WS 2 hybrids.

(a) Normalized Modulus data; (b) Normalized Hardness values.
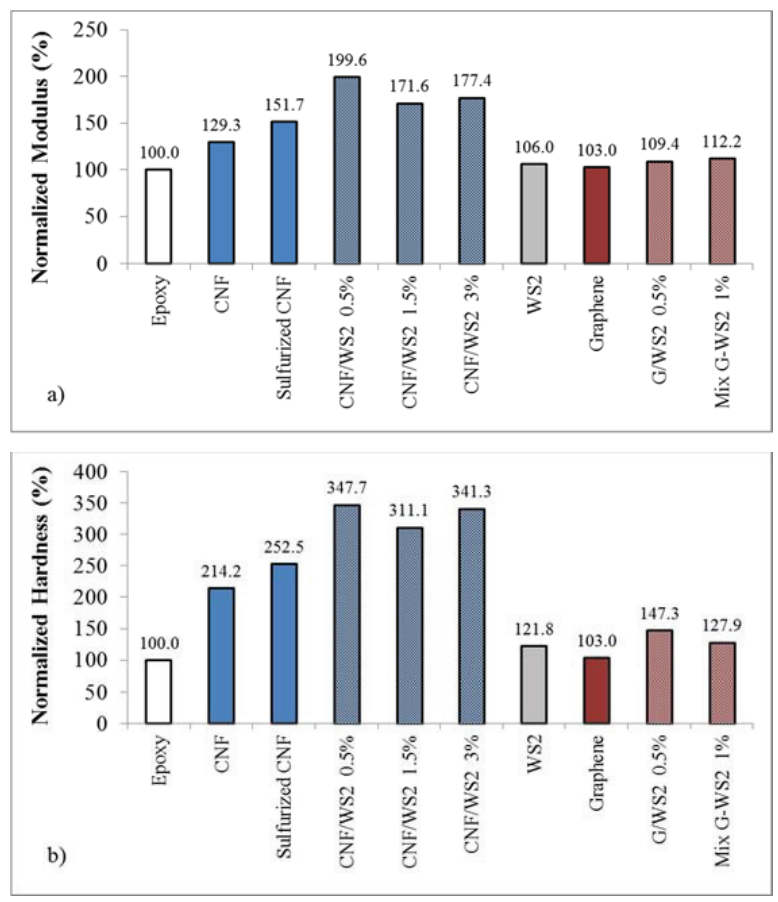

Overall, the epoxy composite samples containing 3D CNF structures along IF-WS 2 created in situ, showed drastic improvements in the Young's modulus and hardness values by the use of only $1 \%$ hybrid weight loadings. The carbon nanofiber inclusions seem to have a much greater impact in the mechanical properties of the composite than the graphene based counterparts for similar IF loadings. 
The values for modulus and hardness improvements over the bare polymer mentioned above for $\mathrm{CNF} / \mathrm{IF}-\mathrm{WS}_{2}$ composites surpass the ones encountered for other hybrid polymeric nanocomposites incorporating IF-WS 2 nanoparticles and a carbon phase [43-45]. However, in order to strictly compare mechanical properties for diverse fillers and polymeric matrices, data gathering should be performed under the same conditions and similar experimental setup to be valid. In particular, the use of nanoindentation instead of DMA tends to produce different values (usually larger for the former technique), as recently corroborated by Flores et al. [67]. Thus, comparison between the two techniques outcomes is not adequate given that no correlation between them exist to date.

\section{Experimental Section}

\subsection{Carbon Nanofiber Hybrids/IF-WS}

The amounts of IF-WS 2 included into CNF were determined by the knowledge gained in previous studies of composites by different groups [14,15,19], and consistent with the intent of generate lightweight materials, making the less dense carbon nanostructure component the most abundant phase. The multistep process employed to generate in situ structures is depicted in Figure 10.

Figure 10. $\mathrm{CNF} / \mathrm{IF}-\mathrm{WS}_{2}$ preparation steps. Ni nanoparticles and $\mathrm{WO}_{3}$ precursors were combined in solution using sonication in ethanol, dried and placed in a crucible inside a quartz tube of a tubular furnace. The mixture was then exposed to diverse environments and thermal treatments to generate Carbon Nanofiber/tungsten disulfide hybrids (CNF/IF-WS 2 ).

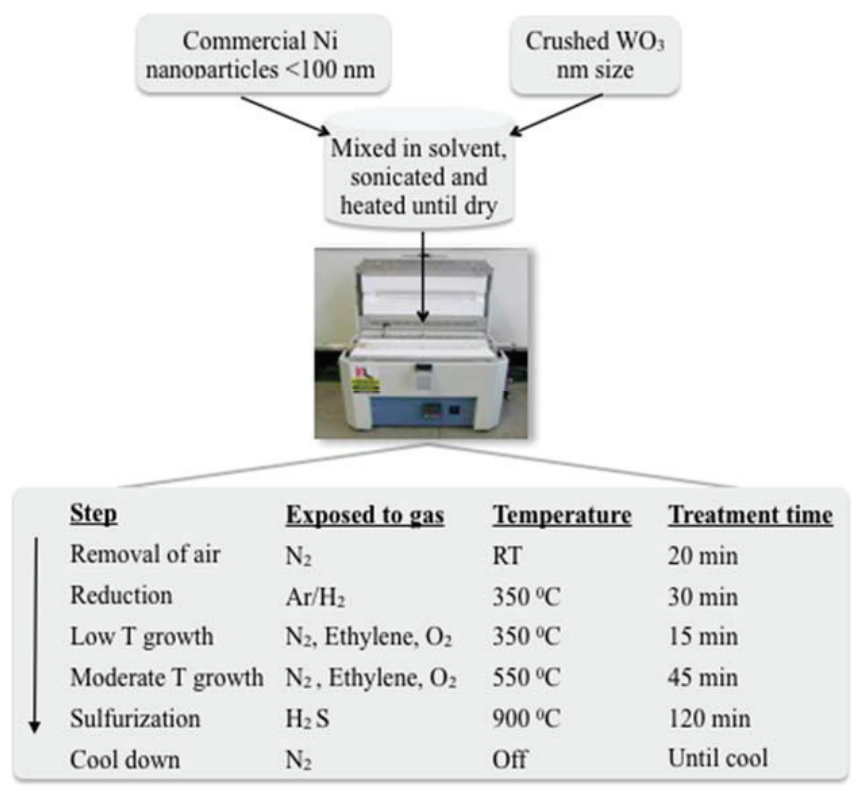


The gas flow rates employed for each step were: $300 \mathrm{sccm} \mathrm{N}$ for air removal, $37 \mathrm{sccm}$ of $\mathrm{Ar} / \mathrm{H}_{2}$ (93\%/7\%) for reduction, a mixture of $44 \mathrm{sccm} \mathrm{N} / 15 \mathrm{sccm}$ Ethylene/2 $\mathrm{sccm} \mathrm{O}_{2}$ for fiber growth (both low temperature and high), a mixture of $150 \mathrm{sccm} \mathrm{N}_{2} / 15 \mathrm{sccm} \mathrm{H}_{2} \mathrm{~S}$ for sulfurization and $300 \mathrm{sccm} \mathrm{N} \mathrm{N}_{2}$ during the cool down process.

\section{2. $\mathrm{IF}-\mathrm{WS}_{2} /$ Graphene Hybrids}

The generation of graphene was performed in all cases starting from graphite flakes, which were oxidized to form graphite oxide (GO) following a modification of the process developed by Marcano et al. [68]. The GO was then used either as (i) dispersion with $\mathrm{WO}_{3}$ nanoparticles and then exfoliated at high temperature with urea as expansion-reducing agent (to generate Graphene/ $\mathrm{WO}_{3}$ ) followed by a sulfurization step using $\mathrm{H}_{2} \mathrm{~S}$ atmosphere (to obtain Graphene/IF- $\mathrm{WS}_{2}$ ) or (ii) exfoliated at high temperature with urea to form graphene, which was then mixed with $\mathrm{WO}_{3}$ and the mixture sulfurized (this step rendered Graphene/IF-WS 2 ). The processes described are depicted in Figure 11.

Figure 11. Synthesis protocol followed to generate Graphene/IF-WS 2 hybrids from GO. Initial precursors (graphite flakes, acids and oxidant) will render graphite oxide. Protocols followed after $\mathrm{GO}$ generation (i) mixture of $\mathrm{GO}$ with $\mathrm{WO}_{3}$ followed by exfoliation and sulfurization and (ii) Production of graphene through GO thermal exfoliation, mixture with $\mathrm{WO}_{3}$ and sulfurization of mixture.

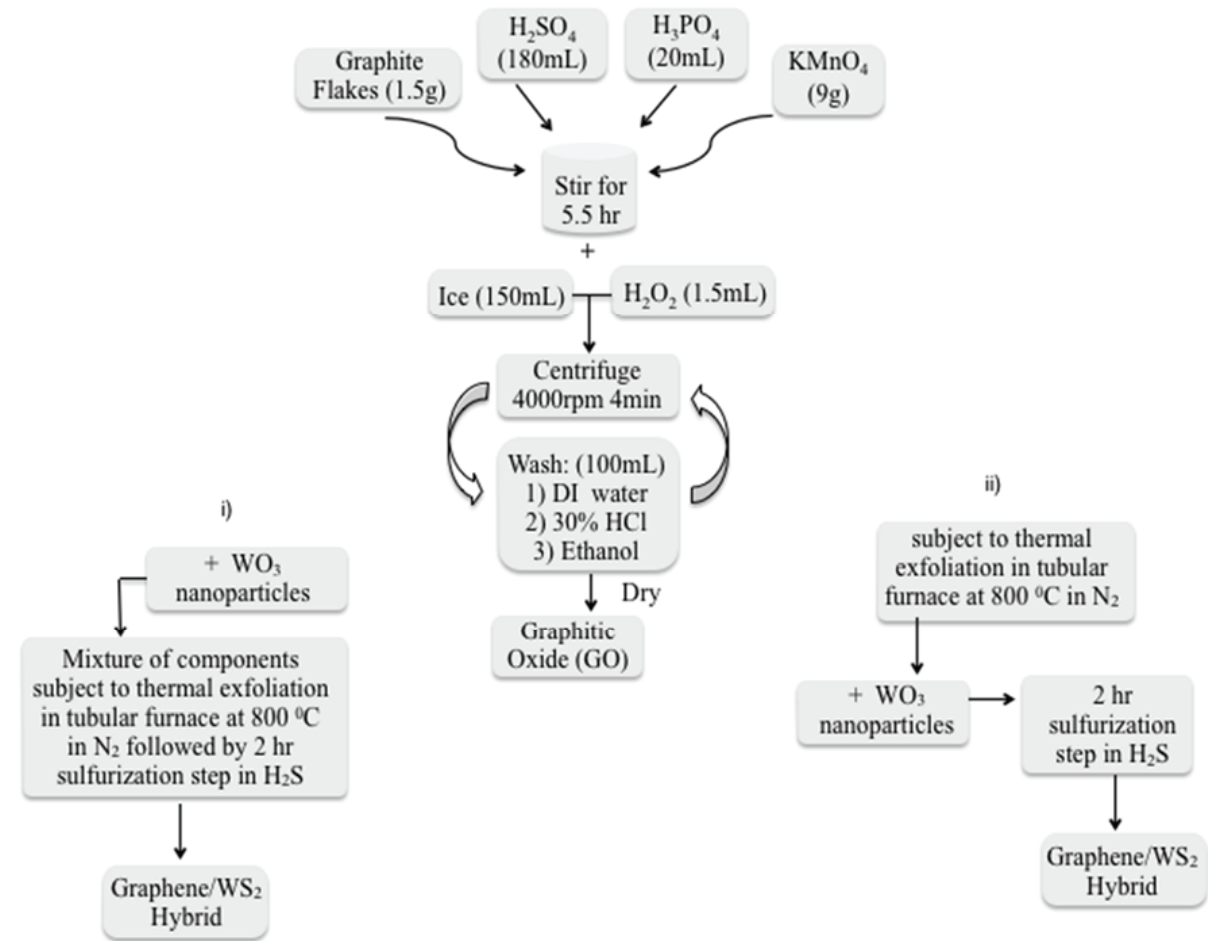




\subsection{Epoxy Composites}

We employed commercially available SpeciFix-20 (Struers, Ballerup, Denmark) as the epoxy resin, which consists of a resin and a hardening agent (26:5 ratio). Unlike some epoxies that require heating or pressure, this epoxy is designed to cure under atmospheric conditions. Further, it has a working period of $60 \mathrm{~min}$, allowing time for the sample to be handled and nanocomposite added and mixed prior to the onset of curing. A loading of $1 \%$ nanoparticles to $99 \%$ epoxy by weight was selected for testing. The composition of the filler materials, the hybrids and individual phases described in previous sections, is included in Table 1 below. The filler components were added to the uncured resin using sonication and the composite was left for a period of at least 24 hours to cure into a hard puck. After the nanoparticles were successfully embedded into the epoxy matrix, the surface of the pucks was mechanically polished to remove scratches and imperfections and then transferred to the nanoindentor.

Table 1. Filler compositions used for epoxy composites (first column).

\begin{tabular}{|c|c|c|c|}
\hline $\begin{array}{l}\text { Sample ID (IF-WS, nominal } \\
\text { composition - filler only) }\end{array}$ & $\begin{array}{l}\text { ICP value } \\
\mathrm{CNF}\end{array}$ & $\begin{array}{l}\text { ICP value } \\
\mathrm{Ni}\end{array}$ & $\begin{array}{l}\text { ICP value } \\
\text { IF-WS2 }\end{array}$ \\
\hline $\mathrm{CNF}$ & $96.8 \%$ & $3.2 \%$ & \\
\hline $\mathrm{CNF} / \mathrm{IF}-\mathrm{WS}_{2}(3 \%)$ & $94 \%$ & $3 \%$ & $3 \%$ \\
\hline $\mathrm{CNF} / \mathrm{IF}-\mathrm{WS}_{2}(1.5 \%)$ & $94.6 \%$ & $3.2 \%$ & $1.6 \%$ \\
\hline $\mathrm{CNF} / \mathrm{IF}-\mathrm{WS}_{2}(0.5 \%)$ & $96.4 \%$ & $2.9 \%$ & $0.7 \%$ \\
\hline IF-WS $2(100 \%)$ & & & $100 \%$ \\
\hline $\begin{array}{l}\text { Sample ID (IFWS, nominal } \\
\text { composition-filler only) }\end{array}$ & Graphene & & IF-WS2 \\
\hline Graphene & $100 \%$ & & \\
\hline $\mathrm{G} / \mathrm{IF}-\mathrm{WS} \mathrm{S}_{2} 0.5 \%$ & $99.5 \%$ & & $0.5 \%$ \\
\hline Physical Mix G/IF-WS $21 \%$ & $99 \%$ & & $1 \%$ \\
\hline & $\begin{array}{l}\text { Composite } \\
\text { Epory } \\
6 \text { Nanostructu }\end{array}$ & Neat Epoxy & \\
\hline
\end{tabular}

\subsection{Characterization Methods}

In order to examine the microstructure of the hybrid specimens the samples were analyzed using a Zeiss Neon 40 High Resolution Scanning Electron Microscope (SEM). Images were acquired at diverse magnifications while the microscope was operated at 10 or $20 \mathrm{kV}$. Energy Dispersive 
Spectroscopy (EDS) experiments were conducted in conjunction with the SEM using the EDAX equipment with an Apollo 10 silicon drift detector (SDD). Data was collected and analyzed using Genesis Spectrum software.

A Netzsch STA 449 FE Jupiter, operated in a Temperature Programmed Oxidation (TPO) mode, was used to study the thermal stability of the samples. The samples were exposed to an $\mathrm{Ar} / \mathrm{O}_{2}$, $80 \% / 20 \%$ atmosphere, with a total flow of $120 \mathrm{~mL}$ minute ${ }^{-1}$, from $\mathrm{RT}$ to $1000{ }^{\circ} \mathrm{C}$ at a heating rate of $10{ }^{\circ} \mathrm{C}$ minute ${ }^{-1}$.

The XRD utilized was a Philips 1830 PAnalytical X-ray Diffractometer. The X-ray tube contained a copper source and the X-rays utilized had a primary wavelength, or K-Alpha, of $1.54 \AA$. The samples were placed into a silicon low background sample holder and the diffraction patterns recorded between $5-70^{\circ}$ ( 2 theta) with 0.020 degrees step size and one second per step.

A JEOL 2010F FASTEM field emission gun scanning transmission electron microscope (STEM/TEM) equipped with Gatan GIF image filtering system was employed. Samples were prepared by dispersing the powders in a few $\mathrm{ml}$ of ethanol and a drop of the dispersion was placed in a copper holey-carbon TEM grid where the ethanol was allowed to evaporate.

A Perkin Elmer ICP 5300 DV-AES Inductive Coupled Plasma Emission Spectrometer, was used to determine the elemental composition of the carbon nanofiber base filler materials before their addition to the epoxy matrix (hybrids $\mathrm{CNF} / \mathrm{IF}-\mathrm{WS}_{2}$ ).

Brunauer Emmet Teller (BET) surface area analysis was performed employing a Quantachrome Nova 4200 . A $300{ }^{\circ} \mathrm{C}$ degas step was conducted prior to the analysis; samples were then allowed to cool down to room temperature and then transferred to the analysis station. The measurements were done using nitrogen atmosphere.

Nanoindentation was used to measure the composite mechanical properties (elastic modulus and hardness) of epoxy composites filled with mixtures of CNFs and IF-WS 2 particles. The samples were prepared by mixing the specified amounts of nanophase material with Struers Speci-Fix 20 two-part epoxy in a $28 \mathrm{~mm}$ diameter mold and then allowing the mixture to cure for $24 \mathrm{~h}$. After curing, the surface of the epoxy composite was polished using standard metallographic techniques, including diamond suspension polishing using suspended aluminum oxide particles of $1 \mu \mathrm{m}$ and $0.05 \mu \mathrm{m}$ diameters. The indentations were performed using an Agilent G200 nanoindenter. We performed two types of experiments with this instrument.

The first experiment was a quasi-static indentation to a set depth, $2 \mu \mathrm{m}$ for all samples. Other indentation parameters can be found in Table 2. This experiment used a diamond, Berkovich indenter tip with a nominal tip radius of $150 \mathrm{~nm}$, calibrated using a fused silica standard. A grid of 20 indentation points spaced by $50 \mu \mathrm{m}$ was measured for each epoxy nanocomposite. The Young's modulus and hardness were calculated using the approach of Oliver and Pharr [69,70].

The second experiment was dynamic mechanical analysis using a $50 \mu \mathrm{m}$ diameter flat punch. This experiment allowed the measurement of both the storage and loss moduli of the epoxy composites. These measurements were performed for five frequency values between $1 \mathrm{~Hz}$ and $45 \mathrm{~Hz}$ on the neat epoxy, CNF and IF-WS2 samples. Other parameters for the measurement can be found in Table 3. A grid of 20 measurements with a $100 \mu \mathrm{m}$ separation between indentations was used for 
each specimen. The storage modulus, loss modulus, and $\tan \delta$ properties for each specimen were calculated using the measurement parameters in Table 3 and the methods of Hay and Herbert [71].

Table 2. Parameters used for quasi-static indentation measurements.

\begin{tabular}{cl}
\hline Depth limit & $2000 \mathrm{~nm}$ \\
Strain rate during loading & $0.08 / \mathrm{s}$ \\
Maximum allowable drift rate & $0.05 \mathrm{~nm} / \mathrm{s}$ \\
Peak hold time & $10 \mathrm{~s}$ \\
Assumed Poisson's ratio & 0.40 \\
\% to unload & 90 \\
\% Unload in stiffness calculation & 50 \\
\hline
\end{tabular}

Table 3. Parameters used for dynamic mechanical analysis.

\begin{tabular}{cc}
\hline Flat punch diameter & $50 \mu \mathrm{m}$ \\
Assumed Poisson's ratio & 0.40 \\
Pre-compression depth & $2 \mu \mathrm{m}$ \\
Oscillation amplitude & $50 \mathrm{~nm}$ \\
\hline
\end{tabular}

\section{Conclusions}

Novel hybrid CNF/IF-WS 2 with diverse IF loadings were generated using an in situ protocol that allowed the integration of the two phases into a tridimensional architecture, producing homogeneous dispersions at the nanoscale in the absence of a polymeric matrix. CNF 3D structures loaded with IF-WS 2 could only be fabricated using a two stage process that involved: (a) the carbon nanofiber growth from a mixture of metal catalyst with tungsten oxide nanoparticles, using ethylene as carbon source and moderate temperatures to render $\mathrm{CNF} / \mathrm{WO}_{3}$, followed by (b) the sulfurization of the sample to convert the tungsten precursor into IF-WS 2 .

In contrast, Graphene/IF-WS 2 hybrids were easily obtained either by mixing graphene and tungsten oxide followed by a sulfurization step or by direct dispersion of the layered graphene structure with existing IF particles using solvents.

The thermal stability of the CNF/IF-WS2 hybrid samples is much higher than those observed for IF-WS 2 by itself or mixed with polymeric components.

Epoxy composites with 1\% weight loadings of hybrid CNF/IF-WS 2 showed drastic improvements in the Young's modulus and hardness values, with approximately 100 and $250 \%$ increase respectively, over the bare epoxy values. The CNF/IF-WS 2 inclusions seem to have a much greater impact in the mechanical properties of the composite than the Graphene/IF-WS 2 based counterparts.

\section{Acknowledgments}

The work depicted in this manuscript has been possible with the support of the Office of Naval Research, Force Protection Thrust, Code 30. We appreciate the help of Dr. Abdul-Mehdi S. Ali from University of New Mexico, who conducted the analysis of the filler components elemental analysis 
by ICP. Our team is thankful for the HRTEM analysis of IF-WS 2 particles conducted by JEOL, USA Inc.

\section{Conflicts of Interest}

The authors declare no conflict of interest.

\section{References}

1. Margulis, L.; Tenne, R.; Iijima, S. Nucleation of $\mathrm{WS}_{2}$ fullerenes at room temperature. Microsc. Microanal. Microstruct. 1996, 7, 87-89.

2. Rapoport, L.; Bilik, Y.; Feldman, Y.; Homyonfer, M.; Cohen, S.; Tenne, R. Hollow Nanoparticles of $\mathrm{WS}_{2}$ as potential solid-state lubricants. Nature 1997, 387, 791-793.

3. Eidelman, O.; Friedman, H.; Tenne, R. Metallic films with fullerene-Like $\mathrm{WS}_{2}\left(\mathrm{MoS}_{2}\right)$ nanoparticles: Self-lubricating coatings with potential applications fullerene-like nanoparticles. In Technological Innovations in Sensing and Detection of Chemical, Biological, Radiological, Nuclear Threats and Ecological Terrorism; Springer: Dordrecht, Netherlands, 2012; pp. 59-67.

4. Rapoport, L.; Fleischer, N.; Tenne, R. Applications of $\mathrm{WS}_{2}\left(\mathrm{MoS}_{2}\right)$ inorganic nanotubes and fullerene-like nanoparticles for solid lubrication and for structural nanocomposites. J. Mater. Chem. 2005, 15, 1782-1788.

5. Drummond, C.; Alcantar, N.; Israelachvili, J.; Tenne, R.; Golan, Y. Microtribology and friction-induced material transfer in $\mathrm{WS}_{2}$ nanoparticle additives. Adv. Funct. Mater. 2001, 11, 348-354.

6. Rothschild, A.; Cohen, S.; Tenne, R. WS 2 nanotubes as tips in scanning probe microscopy. Appl. Phys. Lett. 1999, 75, 4025-4027.

7. Dominko, R.; Arčon, D.; Mrzel, A.; Zorko, A.; Cevc, P.; Venturini, P.; Gaberscek, M.; Remskar, M.; Mihailovic, D. Dichalcogenide nanotube electrodes for li-ion batteries. Adv. Mater. 2002, 14, 1531-1534.

8. Cheng, F.Y.; Chen, J.; Gou, X.L. $\mathrm{MoS}_{2} / \mathrm{Ni}$ Nanocomposites as catalysts for hydrodesulfurization of thiophene and thiophene derivatives. Adv. Mater. 2006, 18, 2561-2564.

9. Wang, Y.; Shi, Z.; Huang, Y.; Ma, Y.; Wang, C.; Chen, M.; Chen, Y. Supercapacitor devices based on graphene materials. J. Phys. Chem. 2009, 113, 13103-13107.

10. Zhu, Y.; Sekine, T.; Brigatti, K.; Firth, S.; Tenne, R.; Rosentsveig, R.; Kroto, H.; Walton, D. Shock-wave resistance of $\mathrm{WS}_{2}$ nanotubes. J. Am. Chem. Soc. 2003, 125, 1329-1333.

11. Zhu, Y.; Sekine, T.; Li, Y.; Wang, W.; Fay, M.; Edwards, H.; Brown, P.; Fleischer, N.; Tenne, R. $\mathrm{WS}_{2}$ and $\mathrm{MoS}_{2}$ Inorganic fullerenes - super shock absorbers at very high pressures. Adv. Mater. 2005, 17, 1500-1503.

12. Zhu, Y.; Sekine, T.; Li, Y.; Fay, M.; Zhao, Y.; Poa, C.; Wang, W.; Roe, M.; Brown, P.; Fleischer, N.; et al. Shock-absorbing and failure mechanisms of $\mathrm{WS}_{2}$ and $\mathrm{MoS}_{2}$ nanoparticles with fullerene-like structures under shock wave pressure. J. Am. Chem. Soc. 2005, 127, $16263-16272$. 
13. Volkova, E.I.; Jones, I.A.; Brooks, R.; Zhu, Y.; Bichoutskaia, E. Meso-scale Modelling of Shock Wave Propagation in a SiC/Al nanocomposite reinforced with $\mathrm{WS}_{2}$-inorganic fullerene nanoparticles. Compos. Struct. 2013, 96, 601-605.

14. Tehrani, M.; Luhrs, C.C.; Al-Haik, M.S.; Trevino, J.; Zea, H. Synthesis of $\mathrm{WS}_{2}$ nanostructures from the reaction of $\mathrm{WO}_{3}$ with $\mathrm{CS}_{2}$ and mechanical characterization of $\mathrm{WS}_{2}$ nanotube composites. Nanotechnology 2011, 22, 285714.

15. Naffakh, M.; Diez-Pascual, A.M.; Marco, C.; Ellis, G.J.; Gomez-Fatou, M.A. Opportunities and challenges in the use of inorganic fullerene-like Nanoparticles to Produce Advanced Polymer Nanocomposites. Prog. Polym. Sci. 2013, 38, 1163-1231.

16. Naffakh, M.; Diez-Pascual, A.M.; Gomez-Fatou, M.A. New Hybrid nanocomposites containing carbon nanotubes, inorganic fullerene-like $\mathrm{WS}_{2}$ nanoparticles and poly(ether ether ketone) (PEEK). J. Mater. Chem. 2011, 21, 7425-7433.

17. Naffakh, M.; Diez-Pascual, A.M.; Marco, C.; Gomez, M.A.; Jimenez, I. Novel melt-processable poly (ether ether ketone) (PEEK)/inorganic fullerene-like $\mathrm{WS}_{2}$ nanoparticles for critical applications. J. Phys. Chem. B 2010, 114, 11444-11453.

18. Diez-Pascual, A.M.; Naffakh, M.; Marco, C.; Ellis, G. Rheological and tribological properties of carbon nanotube/thermoplastic nanocomposites incorporating inorganic fullerene-Like $\mathrm{WS}_{2}$ Nanoparticles. J. Phys. Chem. 2012, 116, 7959-7969.

19. Diez-Pascual, A.M.; Naffakh, M. Mechanical and thermal behaviour of isotactic polypropylene reinforced with inorganic fullerene-like $\mathrm{WS}_{2}$ nanoparticles: Effect of Filler Loading and Temperature. Mater. Chem. Phys. 2013, 141, 979-989.

20. Ma, T.; Zhang, T.; Gao, P.G.; Zhang, J.C. Synthesis and properties of ultrahigh molecular weight polyethylene/WS 2 nanoparticle fiber for bullet-proof materials. Chin. Sci. Bull. 2013, 58, 945-948.

21. Shneider, M.; Dodiuk, H.; Tenne, R.; Kenig, S. Nanoinduced morphology and enhanced properties of epoxy containing tungsten disulfide nanoparticles. Polym. Eng. Sci. 2013, 53, 2624-2632.

22. Shneider, M.; Rapoport, L.; Moshkovich, A.; Dodiuk, H.; Kenig, S.; Tenne, R.; Zak, A. Tribological performance of the epoxy-based composite reinforced by $\mathrm{WS}_{2}$ fullerene-like nanoparticles and nanotubes. Phys. Status Solidi A 2013, 210, 2298-2306.

23. Zohar, E.; Baruch, S.; Shneider, M.; Dodiuk, H.; Kenig, S.; Tenne, R.; Wagner, H.D. The Effect of $\mathrm{WS}_{2}$ nanotubes on the properties of epoxy-based nanocomposites. J. Adhes. Sci. Technol. 2011, 25, 1603-1617.

24. Shneider, M.; Dodiuk, H.; Kenig, S.; Tenne, R. The Effect of Tungsten Sulfide Fullerene-Like Nanoparticles on the toughness of epoxy adhesives. J. Adhes. Sci. Technol. 2010, 24, 1083-1095.

25. Lalwani, G.; Kwaczala, A.T.; Kanakia, S.; Patel, S.C.; Judex, S.; Sitharaman, B. Fabrication and characterization of three-dimensional macroscopic all-carbon scaffolds. Carbon 2013, 53, $90-100$.

26. Franklin, N.; Dai, H. An enhanced CVD approach to extensive nanotube networks with directionality. Adv. Mater. 2000, 12, 890-894. 
27. Calvo, M.; Garcia, R.; Arenillas, A.; Suarez, I.; Moinelo, S. Carbon foams from coals. A preliminary study. Fuel 2005, 84, 2184-2189.

28. Calvo, M.; Garcia, R.; Moinelo, S.R. Carbon foams from different coals. Energy Fuels 2008, 22, 3376-3383.

29. Chen, C.; Kennel, E.; Stiller, A.; Stansberry, P.; Zondlo, J. Carbon foam derived from various precursors. Carbon 2006, 44, 1535-1543.

30. Chakrapani, N.; Wei, B.; Carrillo, A.; Ajayan, P.; Kane, R. Capillarity-driven assembly of two-dimensional cellular carbon nanotube foams. Proc. Natl. Acad. Sci. USA 2004, 101, 4009-4012.

31. Chakrapani, N.; Wei, B.; Carrillo, A.; Ajayan, P.; Kane, R. Capillarity-driven assembly of cellular carbon nanotube foams. Abstr. Pap. Am. Chem. Soc. 2004, 228, U481-U481.

32. Gallego, N.; Klett, J. Carbon Foams for Thermal Management. Carbon 2003, 41, 1461-1466.

33. Kaur, S.; Ajayan, P.M.; Kane, R.S. Design and characterization of three-dimensional carbon nanotube foams. J. Phys. Chem. B 2006, 110, 21377-21380.

34. Dai, L.; Patil, A.; Gong, X.; Guo, Z.; Liu, L.; Liu, Y.; Zhu, D. Aligned nanotubes. Chemphyschem 2003, 4, 1150-1169.

35. Atwater, M.A.; Mousavi, A.K.; Leseman, Z.C.; Phillips, J. Direct synthesis and characterization of a nonwoven structure comprised of carbon nanofibers. Carbon 2013, 57, 363-370.

36. Safdari, M.; Al-Haik, M.S. Optimization of stress wave propagation in a multilayered elastic/viscoelastic hybrid composite based on carbon fibers/carbon nanotubes. Polym. Compos. 2012, 33, 196-206.

37. Khalid, W.; Ali, M.S.M.; Dahmardeh, M.; Choi, Y.; Yaghoobi, P.; Nojeh, A.; Takahata, K. High-aspect-ratio, free-form patterning of carbon nanotube forests using micro-electro-discharge machining. Diamond Relat. Mater. 2010, 19, 1405-1410.

38. Qiu, L.; Liu, J.Z.; Chang, S.L.Y.; Wu, Y.; Li, D. Biomimetic superelastic graphene-based cellular monoliths. Nat. Commun. 2012, 3, 1241.

39. Geim, A.K.; Novoselov, K.S. The rise of graphene. Nat. Mater. 2007, 6, 183-191.

40. Stankovich, S.; Dikin, D.A.; Dommett, G.H.B.; Kohlhaas, K.M.; Zimney, E.J.; Stach, E.A.; Piner, R.D.; Nguyen, S.T.; Ruoff, R.S. Graphene-based composite materials. Nature 2006, 442, 282-286.

41. Naffakh, M.; Diez-Pascual, A.M.; Marco, C.; Ellis, G. Morphology and thermal properties of novel poly (phenylene sulfide) hybrid nanocomposites based on single-walled carbon nanotubes and inorganic fullerene-like $\mathrm{WS}_{2}$ nanoparticles. J. Mater. Chem. 2012, 22, 1418-1425.

42. Diez-Pascual, A.M.; Naffakh, M.; Gomez-Fatou, M.A. Mechanical and electrical properties of novel poly(ether ether ketone)/carbon nanotube/inorganic fullerene-like $\mathrm{WS}_{2}$ hybrid nanocomposites: Experimental measurements and theoretical predictions. Mater. Chem. Phys. 2011, 130, 126-133.

43. Diez-Pascual, A.M.; Naffakh, M. Inorganic Nanoparticle-Modified Poly(Phenylene Sulphide)/Carbon Fiber Laminates: Thermomechanical Behaviour. Materials 2013, 6, 3171-3193. 
44. Diez-Pascual, A.M.; Naffakh, M. Tuning the properties of carbon fiber-reinforced poly (phenylene sulphide) laminates via incorporation of inorganic nanoparticles. Polymer 2012, 53, 2369-2378.

45. Diez-Pascual, A.M.; Naffakh, M.; Marco, C.; Ellis, G. Mechanical and electrical properties of carbon nanotube/poly(phenylene sulphide) composites incorporating polyetherimide and inorganic fullerene-like nanoparticles. Compos. Part A 2012, 43, 603-612.

46. Luhrs, C.C.; Daskam, C.D.; Gonzalez, E.; Phillips, J. Fabrication of a Low Density Carbon Fiber Foam and Its Characterization as a Strain Gauge. Materials, submitted for publication, 2014.

47. Moberg, M. Carbon Fiber and Tungsten Disulfide Nanoscale Architectures for Armor Applications. MS Thesis, Naval Postgraduate School, June 2012.

48. Feldman, Y.; Frey, G.; Homyonfer, M.; Lyakhovitskaya, V.; Margulis, L.; Cohen, H.; Hodes, G.; Hutchison, J.; Tenne, R. Bulk synthesis of inorganic fullerene-like $\mathrm{MS}_{2}(\mathrm{M}=\mathrm{Mo}, \mathrm{W})$ from the respective trioxides and the reaction mechanism. J. Am. Chem. Soc. 1996, 118, 5362-5367.

49. Tenne, R.; Homyonfer, M.; Feldman, Y. Nanoparticles of layered compounds with hollow cage structures (inorganic fullerene-like structures). Chem. Mater. 1998, 3225-3238.

50. Tenne, R.; Remskar, M.; Enyashin, A.; Seifert, G. Inorganic nanotubes and fullerene-like structures (IF). Carbon Nanotubes 2008, 111, 631-671.

51. Bar-Sadan, M.; Kaplan-Ashiri, I.; Tenne, R. Inorganic fullerenes and nanotubes: Wealth of materials and morphologies. Eur. Phys. J.-Spec. Top. 2007, 149, 71-101.

52. Wiesel, I.; Arbel, H.; Albu-Yaron, A.; Popovitz-Biro, R.; Gordon, J.M.; Feuermann, D.; Tenne, R. Synthesis of $\mathrm{WS}_{2}$ and $\mathrm{MoS}_{2}$ Fullerene-Like Nanoparticles from Solid Precursors. Nano Res. 2009, 2, 416-424.

53. Bastide, S.; Borra, J.; Duphil, D.; Levy-Clement, C. Synthesis of inorganic fullerenes and nanoboxes of $\mathrm{MoS}_{2}$ and $\mathrm{WS}_{2}$ by spray pyrolysis. Abstr. Pap. Am. Chem. Soc. 2004, 228, U482-U482.

54. Zink, N.; Pansiot, J.; Kieffer, J.; Therese, H.A.; Panthofer, M.; Rocker, F.; Kolb, U.; Tremel, W. Selective synthesis of hollow and filled fullerene-like (IF) $\mathrm{WS}_{2}$ nanoparticles via metal-organic chemical vapor deposition. Chem. Mater. 2007, 6391-6400.

55. Brooks, D.; Douthwaite, R.; Brydson, R.; Calvert, C.; Measures, M.; Watson, A. Synthesis of inorganic fullerene $\left(\mathrm{MS}_{2}, \mathrm{M}=\mathrm{Zr}\right.$, Hf and $\left.\mathrm{W}\right)$ phases using $\mathrm{H}_{2} \mathrm{~S}$ and $\mathrm{N}_{2} / \mathrm{H}_{2}$ microwave-induced plasmas. Nanotechnology 2006, 17, 1245-1250.

56. Tenne, R. Inorganic nanotubes and fullerene-like nanoparticles. Nat. Nanotechnol. 2006, 1, 103-111.

57. Luhrs, C.C.; Garcia, D.; Tehrani, M.; Al-Haik, M.; Taha, M.R.; Phillips, J. Generation of carbon nanofilaments on carbon fibers at 550 degrees C. Carbon 2009, 47, 3071-3078.

58. Pierson, H.O. Handbook of Carbon, Graphite, Diamond, and Fullerenes; Noyes Publications: Park Ridge, NJ, USA, 1993.

59. Fleet, M.E. Crystal-Structure of Heazlewoodite, and Metallic Bonds in Sulfide Minerals. Am. Mineral. 1977, 62, 341-345.

60. Parise, J.B. Structure of Hazelwoodite $\left(\mathrm{Ni}_{3} \mathrm{~S}_{2}\right)$. Acta Crystallogr. Sect. B 1980, 36, 1179-1180. 
61. Villars, P.; Cenzual, K. Landolt-Bornstein-Group III Condensed Matter; Springer: Berlin, Germany, 2010.

62. Xu, F.; Almeida, T.P.; Chang, H.; Xia, Y.; Wears, M.L.; Zhu, Y. Multi-walled carbon/IF-WS 2 nanoparticles with improved thermal properties. Nanoscale 2013, 5, 10504-10510.

63. Schuffenhauer, C.; Wildermuth, G.; Felsche, J.; Tenne, R. How stable are inorganic fullerene-like particles. Thermal analysis (STA) of inorganic fullerene-like $\mathrm{NbS}_{2}, \mathrm{MoS}_{2}$, and $\mathrm{WS}_{2}$ in oxidizing and inert atmospheres in comparison with the bulk material. Phys. Chem. Chem. Phys. 2004, 6, 3991-4002.

64. Chang, L.; Yang, H.; Fu, W.; Yang, N.; Chen, J.; Li, M.; Zou, G.; Li, J. Synthesis and thermal stability of W/WS 2 inorganic fullerene-like nanoparticles with core-shell structure. Mater. Res. Bull. 2006, 41, 1242-1248.

65. Dutta, A.; Penumadu, D.; Files, B. Nanoindentation testing for evaluating modulus and hardness of single-walled carbon nanotube-reinforced epoxy composites. J. Mater. Res. 2004, 19, 158-164.

66. Gromowski, K. Glass Breakage—Nickel Sulfide Inclusions; Penn State: State College, PA, USA, 2010.

67. Flores, A.; Naffakh, M.; Diez-Pascual, A.M.; Ania, F.; Gomez-Fatou, M.A. Evaluating the Reinforcement of Inorganic Fullerene-like Nanoparticles in Thermoplastic Matrices by Depth-Sensing Indentation. J. Phys. Chem. C 2013, 117, 20936-20943.

68. Marcano, D.C.; Kosynkin, D.V.; Berlin, J.M.; Sinitskii, A.; Sun, Z.; Slesarev, A.; Alemany, L.B.; Lu, W.; Tour, J.M. Improved Synthesis of Graphene Oxide. ACS Nano 2010, 4, 4806-4814.

69. Oliver, W.; Pharr, G. An Improved Technique for Determining Hardness and Elastic-Modulus using Load and Displacement Sensing Indentation Experiments. J. Mater. Res. 1992, 7, 1564-1583.

70. Pharr, G.; Oliver, W.; Brotzen, F. On the Generality of the Relationship among Contact Stiffness, Contact Area, and Elastic-Modulus during Indentation. J. Mater. Res. 1992, 7, 613-617.

71. Hay, J.; Herbert, E. Measuring the Complex Modulus of Polymers by Instrumented Indentation Testing. Exp. Tech. 2013, 37, 55-61. 


\title{
From Stable ZnO and GaN Clusters to Novel Double Bubbles and Frameworks
}

\author{
Matthew R. Farrow, John Buckeridge, C. Richard A. Catlow, Andrew J. Logsdail, \\ David O. Scanlon, Alexey A. Sokol and Scott M. Woodley
}

\begin{abstract}
A bottom up approach is employed in the design of novel materials: first, gas-phase "double bubble" clusters are constructed from high symmetry, $T_{h}, 24$ and 96 atom, single bubbles of $\mathrm{ZnO}$ and $\mathrm{GaN}$. These are used to construct bulk frameworks. Upon geometry optimization - minimisation of energies and forces computed using density functional theory - the symmetry of the double bubble clusters is reduced to either $\mathrm{C}_{1}$ or $\mathrm{C}_{2}$, and the average bond lengths for the outer bubbles are $1.9 \AA$, whereas the average bonds for the inner bubble are larger for $\mathrm{ZnO}$ than for GaN; $2.0 \AA$ and $1.9 \AA$, respectively. A careful analysis of the bond distributions reveals that the inter-bubble bonds are bi-modal, and that there is a greater distortion for $\mathrm{ZnO}$. Similar bond distributions are found for the corresponding frameworks. The distortion of the $\mathrm{ZnO}$ double bubble is found to be related to the increased flexibility of the outer bubble when composed of $\mathrm{ZnO}$ rather than GaN, which is reflected in their bulk moduli. The energetics suggest that $(\mathrm{ZnO})_{12} @(\mathrm{GaN})_{48}$ is more stable both in gas phase and bulk frameworks than $(\mathrm{ZnO})_{12} @(\mathrm{ZnO})_{48}$ and $(\mathrm{GaN})_{12} @(\mathrm{GaN})_{48}$. Formation enthalpies are similar to those found for carbon fullerenes.
\end{abstract}

Reprinted from Inorganics. Cite as: Farrow, M.R.; Buckeridge, J.; Catlow, C.R.A.; Logsdail, A.J.; Scanlon, D.O.; Sokol, A.A.; Woodley, S.M. From Stable ZnO and GaN Clusters to Novel Double Bubbles and Frameworks. Inorganics 2014, 2, 248-263.

\section{Introduction}

A new class of materials is sought that will support the separation of electrons and holes typically generated during photo-excitation by solar radiation. In this context, heterostructures of $\mathrm{ZnO} / \mathrm{GaN}$ attract particular interest, as such materials have great potential in a wide range of applications from semiconductor optoelectronics to photo-catalysis [1-5].

Previous computational simulations [6-8] have predicted that both $\mathrm{ZnO}$ and $\mathrm{GaN}$, at the nanoscale, form clusters with a bubble architecture that are dramatically different from models cut from their wurtzite bulk structures. Using $\mathrm{ZnO}$ and $\mathrm{SiC}$ as two simple examples, we have shown $[9,10]$ how individual bubbles can combine to form extended framework materials; alternative constructions and the viability (or stability) of similar frameworks using bubbles as building blocks have also been reported [6,9-11]. For framework structures, an increase in density is typically correlated with an enhanced stability, which can be achieved by connecting appropriate building units. In our approach to framework construction, we choose a new type of unit, the so-called "double bubble", that are themselves denser than single-shell bubbles and which are a preferred motif for larger sized clusters [12].

For binary oxide and semiconductor II-VI and III-V materials with a 1-1 stoichiometry, fullerene type structures have been the focus of materials modelling at the nanoscale in the last decade. This 
interest has partly been spurred by reports of synthesis of $(\mathrm{MX})_{n}$ clusters of these materials, where $\mathrm{M}$ denotes metals, or cations, and $\mathrm{X}$ represents anions, with the mass spectra of such systems showing unexpected preference for certain sizes $n$. The preferred values of $n$ are widely known as "magic numbers" [8]. The stability of such clusters has been explained on thermodynamic grounds: the binding energy per formula unit as a function of size having a minimum (i.e., the energy released on cluster formation has a maximum). Alternative explanations have been proposed using: (i) a kinetic argument based on whether the cluster growth or shrinkage is an energetically favourable process; and (ii) a statistical argument: a particular cluster size may be realized in a greater number of atomic configurations compared to others, and therefore is favoured entropically. For any particular experiment, one or a combination of these factors may in fact be relevant.

Considering the atomic structure of stable clusters as a function of increasing size, we recognise an evolution of basic structural units with increasing dimensionality: from 1D—sticks; to 2D — rings and patchworks of rings; and then to 3D units that are initially composed of one layer-a tube or bubble - and then multiple layers, and finally bulk like phases (those that could be stable or metastable on the macroscopic scale). The bubble structures are also denoted in the literature as cages, spheroids, or fullerenes. The above classification is also based on the atomic connectivity or bonding, which increases with increased dimensionality. Using additional definitions given in reference [13], perfect closed bubbles are an important subclass of single walled fullerene-like clusters, in which each atom has three nearest neighbours, and the surface of such fullerenes is composed of a patchwork of hexagonal faces that is wrapped in three dimensions by the introduction of six "defects", or tetragonal faces. The existence and stability of the fullerene-like inorganic structures have been known from both theory and experiment for $\mathrm{BN}, \mathrm{ZnO}$, and $\mathrm{MoS}_{2}$ [14-16]. Perfect bubbles can also include larger patches with an even number of sides complemented by an appropriate number of tetragonal faces. In contrast to carbon fullerenes, pentagonal faces are not realised for the heterogeneous semiconductor class of compound, as they would require formation of $\mathrm{M}-\mathrm{M}$ and/or X-X bonds that are electrostatically unfavourable. Due to the ionic nature of bonding in these materials, the charge disproportionation is not compatible with electron localisation, required for metal-metal bond formation, or hole localisation, which stabilises di-oxygen or di-nitrogen species.

On further size increase (cluster growth), the appearance of layered structures becomes a possibility, in which a smaller sized cluster unit is contained within a larger bubble structure. Indeed, such structures have been discovered in molecular dynamical studies of $\mathrm{ZnS}$, where the smallest is found for $n=60$ : an $n=48$ bubble forms a concentric shell around an $n=12$ sodalite cage [12]. Although both single bubbles have the same high symmetry $T_{h}$ point group, the double bubble can relax into a lower symmetry form depending on the composition. The $T_{h}$ symmetry unit, however, can be stabilised when this unit is used in frameworks that were constructed previously from the individual single layered components.

In this paper, we investigate the different possible atomic structural relaxations of the double bubble and the effect of mixing components of different compositions, for both the individual clusters and the constructed frameworks. 


\section{Construction of Double Bubbles}

\section{High Symmetry Double Bubble Clusters as Secondary Building Units}

We consider the 1-1 compounds that are predicted to have stable (lowest energy for a particular size) and metastable bubble, or fullerene like, structures. Perfect versions of these structures are composed of only three-coordinated atoms, sets of which create rings with an even number of sides that can be visualised as one of the faces of the bubble; an odd number of sides is unlikely as this would require at least one neighbouring pair of vertices of only cations or anions. Except for the smallest sized clusters, in which the curvature of the layer is important, cluster configurations containing one- or two-coordinated atoms are less stable than the perfect bubbles. Another characteristic of the stable bubbles is that, typically, the number of tetragonal rings is minimised (and, to a lesser extent, the distance between these should be maximised), while the number of hexagonal rings is maximised. A layer consisting of only hexagonal rings has no curvature, and therefore a perfect bubble of hexagons would require an infinite number of atoms (i.e., a 2D infinite hexagonal sheet). To obtain a perfect bubble with a finite number of atoms, the sheet requires the introduction of six tetragonal rings (Euler's rule) as each tetragonal ring increases the curvature of the sheet. Increasing the number of tetragonal rings results in open (as opposed to closed) perfect bubbles, which contain much larger rings, e.g., octagonal, assuming the chemistry of the compound does not favour the bonding and coordination required for the formation of cuboids, i.e., cuts from rock salt.

The higher symmetry configurations of the perfect bubbles are typically found to be more stable. As high symmetry cluster structures are only possible for certain sizes, they are not only the stable state for their particular size, but usually have a greater stability than clusters of neighbouring sizes. In our previous studies $[6,9,10]$ we have, therefore, focused our attention on families of high symmetry structures, and, in particular, those with symmetry $T_{h}, T_{d}$ and $T$. These $(\mathrm{MX})_{n}$ structures are found if $n=4\left(T_{d}\right), 12\left(T_{h}\right), 16\left(T_{d}\right), 28(T), 36\left(T_{d}\right), 48\left(T_{h}\right), 64\left(T_{d}\right), \ldots$ Larger $T_{h}$ and $T_{d}$ clusters include $n=108$ and 192 and $n=100,144$ and 196, respectively; examples are shown in Figure 1. These clusters can be visualised as truncated octahedra, where there is one tetragonal ring of the bubble at each of the six truncated corners, and the hexagonal patchworks form the octahedron's faces. In this morphology, the distance between all tetragonal rings is maximised for a given size $n$, and the separation increases monotonically with $n$.

Figure 1. Models of high symmetry $(\mathrm{MX})_{n}$ bubbles with: (a) $n=12$ with symmetry $T_{h}$;

(b) $n=48$ with symmetry $T_{h}$; (c) $n=28,36$ and 48 with octahedra superimposed.
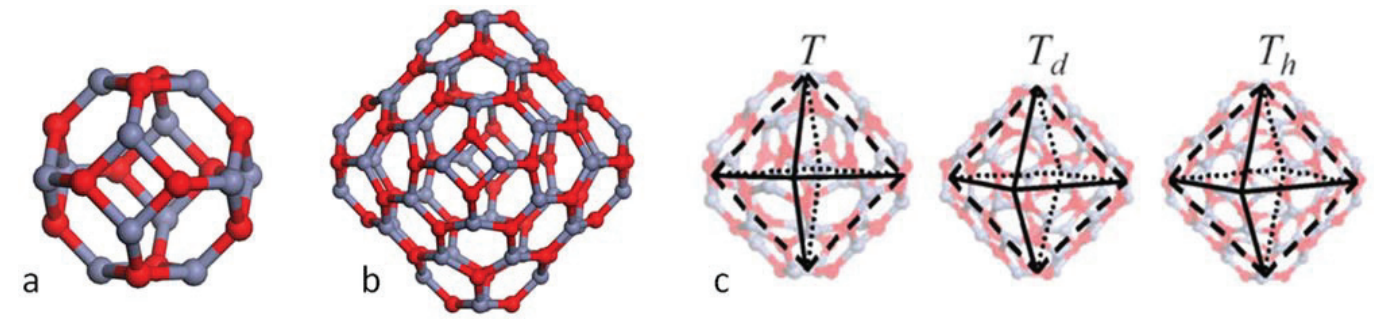
Smaller bubble clusters can be readily generated using an appropriate global optimiser (e.g., one based on Monte Carlo basin hopping [17] or genetic algorithm [18] routines), and once one has determined the relationship between them, the larger bubble clusters can be constructed by simply increasing the number of rows of hexagonal rings in each face or edge of the octahedron. For example, each octahedron's edge of a $T_{d}$ bubble links a side of two tetragonal rings via a "ladder" of $m$ hexagonal rings (one hexagon wide) - see the highlighted ladder in Figure $2 \mathrm{a}$ - with the remaining hexagonal rings completing the faces of the octahedron. Note that the line of the octahedron edge bisects the rings of this ladder and that the tetragonal ring is out of phase with the tetragonal face created by truncating the octahedron. Constructions with $m=0,1,2,3,4$ and 5 corresponds to perfect bubbles at $n=4,16,36,64,100,144$ and 196, respectively. In contrast, each octahedron edge in a $T_{h}$ bubble links the corner of two tetragonal rings via $m+1 \mathrm{M}-\mathrm{X}$ sticks that are separated by $m$ hexagonal rings, forming an alternating linear pattern. Each stick is actually a shared side of two hexagonal rings, with each ring part of a hexagonal patchwork that covers a face of the octahedron. These sticks form a line segment of the octahedron edge (see highlighting in Figure 2b), and this line bisects opposite angles of the hexagonal rings, rather than opposite sides, and the tetragonal rings are in-phase with the tetragonal face created by truncating the octahedron. The smallest $T_{h}$ bubble, $m=0$, or $n=12$, has just one stick between two neighbouring tetragonal rings. The next smallest size $T_{h}$ bubble, $m=1$, or $n=48$, is constructed using two sticks and one hexagonal ring; then, for $m=2$, or $n=108$, there are three sticks and two hexagonal rings. Comparing the growth of the octahedron edges, it is evident why there are more bubbles with $T_{d}$ rather than $T_{h}$ symmetry.

Figure 2. Models of high symmetry $(\mathrm{MX})_{n}$ bubbles with: (a) $n=64$, symmetry $T_{d}$ and the ladder of hexagonal rings, highlighted in yellow, that corresponds to one of the twelve edges of an octahedron; (b) $n=48$, symmetry $T_{h}$ with a fragment that corresponds to one of twelve edges highlighted in yellow; (c) $n=48$, symmetry $T_{h}$ with one of twelve patchworks that correspond to the octahedron side highlighted in purple.
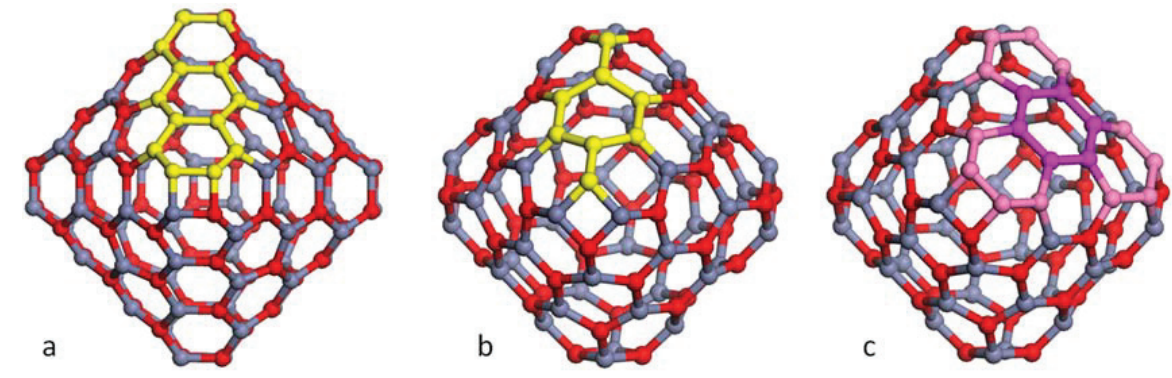

As discussed in the Introduction, framework structures with an increased density are typically more stable $[19,20]$. We therefore choose to investigate double bubbles that are composed of high symmetry perfect bubbles as these are more dense. Two bubbles are combined by inserting the smaller bubble inside the larger; aligned with the same centre of mass and identical direction of orthogonal axes, with each axis passing through the centre of mass and the centre of opposite truncated corners, or tetragonal faces. The rotation of these tetragonal faces about the octahedral axes 
is dependent upon the symmetry of the cluster. When clusters have $T_{h}$ and $T_{d}$ symmetry this rotation is $45^{\circ}$ out-of-phase, and, if one of the bubbles has T symmetry, between $0^{\circ}$ and $45^{\circ}$. For stability, the best match is obtained when the inner and outer bubbles are taken from the set of $T_{h}$ bubbles and the highest density obtained by combining the smallest two of these: $n=12$ (a sodalite cage) and $n=48[10]$.

In the MX bulk phase considered here, the atoms are four-coordinated tetrahedra, so the stability of the double bubbles will improve if $\mathrm{M}-\mathrm{X}$ linkages between layers are found. These linkages can be expected to be located between aligned pairs of hexagonal patchworks that form the faces of the octahedra rather than between the truncated corners. For the $n=60$ double bubble, the inner $n=12$ bubble has one hexagonal ring on each face, whereas the outer $n=48$ bubble is composed of a patchwork of five and a half hexagonal rings; a central hexagonal ring that is linked via one hexagonal ring to each of three nearest tetragonal rings and three hexagonal rings that are shared with neighbouring faces of the octahedron (see Figure 2c). Importantly, the central hexagonal ring can bond with the hexagonal ring of the inner bubble; see Figure 3. Analogous to our structures, experimentally observed cages of boron nitride $(\mathrm{BN}),[15,16]$ and molybdenum sulphide $\left(\mathrm{MoS}_{2}\right)[21,22]$ have been reported to be constructed from four and six (hexagonal) membered ring building units. CdSe cage structures have been experimentally observed to be stable and formed from truncatedoctahedra [23]. DFT calculations on cage structures of CdSe that are similar to our structures have also been reported [24].

Figure 3. Models of the $n=60 T_{h}$ double bubble, with inter-layer links between the inner $n=12$ sodalite cage and the eight hexagonal rings that are in the centres of the octahedron faces of the outer $n=48$ bubble, highlighted using ball-and-sticks rather than line representation for: (a) no bridging links; (b) four bridging links; and (c) all eight bridging links.
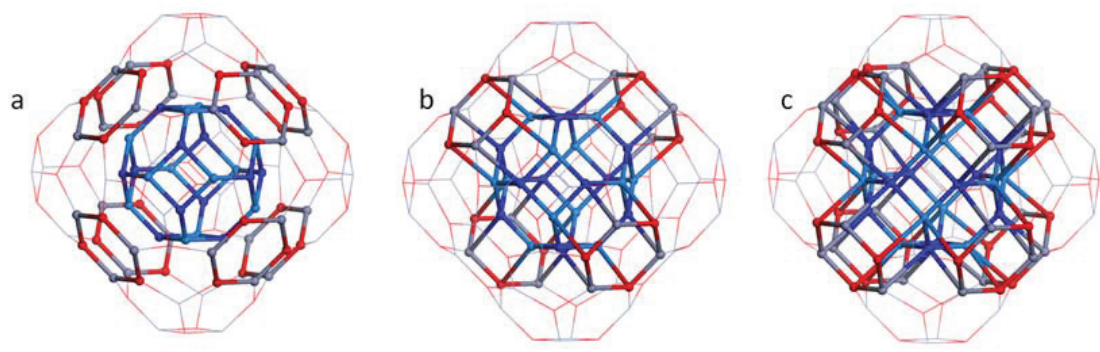

The first framework is constructed from $T_{h}$ bubble (sodalite cage) secondary building units (SBUs) of $(\mathrm{ZnO})_{12}$ and $(\mathrm{GaN})_{12}$, see Figure 1a. As the typical $\mathrm{Zn}-\mathrm{O}$ and $\mathrm{Ga}-\mathrm{N}$ bond lengths are similar (1.98 $\AA$ and $1.95 \AA$ in their ground state wurtzite form), their respective SBUs are also similar in size. Consider each SBU as an octahedron. By corner sharing the octahedra, and assuming an equal number of SBUs for each compound, we construct an fcc, rock-salt like lattice, as shown in Figure 4c. The second framework is constructed from the $n=60 T_{h}$ double bubbles; see Figures 1c and $4 \mathrm{~b}$. Again, imagining each SBU as an octahedron, but rather than corner sharing they are now stacked so that they share edges, each double bubble is surrounded by twelve others (see Figure 4d), 
and each edge of the outer bubble is one bond length from an edge of a neighbouring bubble forming an $n=6$ double ring (a drum) and two $n=2$ rings. Each tetragonal ring of an outer $n=48$ bubble combines with five others to form an $n=12 T_{h}$ bubble, i.e., the void is a sodalite cage. The inner sodalite cage of each double bubble is formed from (i) the same compound and (ii) two compounds, which we alternate.

We start the double bubble construction from two relaxed single bubbles. If the distance between each inner hexagonal ring and its corresponding central hexagonal ring of the outer bubble is approximately a typical $\mathrm{M}-\mathrm{X}$ bond length, then we shall refer to this as an ideal match, and the relaxed double bubble is expected to maintain $T_{h}$ symmetry. Whether there is an ideal match depends on the composition: if the two layers are of the same composition and there is not an ideal match then the inner bubble is too small. The outer eight planes of hexagonal patchworks, or octahedron faces, have more flexibility than the corners. During a geometry relaxation of the double bubble, the central hexagonal ring of each outer patchwork can move inward, maintaining the $T_{h}$ symmetry or, due to the repulsion between neighbouring patchworks, only the central hexagons from alternate patchworks, i.e., four of the eight, move inwards reducing the symmetry to $T$; see Figure 3.

For all $\mathrm{ZnO} / \mathrm{GaN}$ compositions investigated here, $n=60$ double bubble structures of high symmetry ( $T_{h}$ and $T$ ) were constructed and then geometry optimised. Low symmetry structural distortions were allowed in the optimisation process in order to find the lowest energy double bubble configuration.

Figure 4. Ball and stick models of two framework structures. (a) Constructed from $T_{h}$ bubbles of $(\mathrm{GaN})_{12}$ and $(\mathrm{ZnO})_{12} ;$ (b) Constructed from $T_{h}$ double bubbles of $(\mathrm{ZnO})_{48}$ and $(\mathrm{GaN})_{12}$; (c) the same structure as (a) but with each $(\mathrm{GaN})_{12}$ coloured red and each $(\mathrm{ZnO})_{12}$ coloured blue (lighter/darker shades used in the front/back row); (d) the same structure as $(\mathrm{b})$ but with each $(\mathrm{GaN})_{12}$ hidden and each $(\mathrm{ZnO})_{48}$ uniquely coloured.
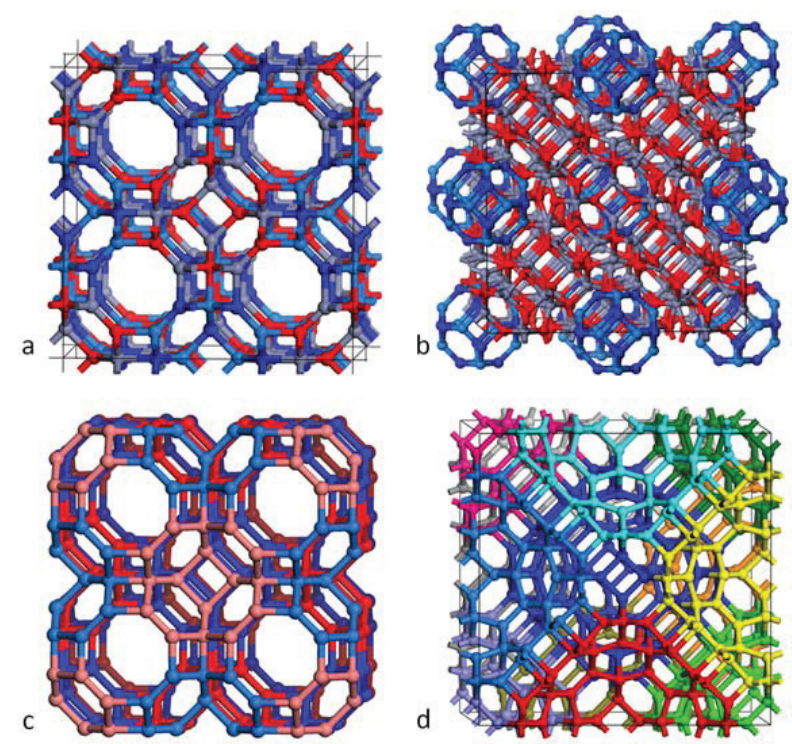


\section{Results and Discussion}

\subsection{Double Bubble Clusters}

With each layer (single bubble) composed of either $\mathrm{ZnO}$ or $\mathrm{GaN}$, there are four possible $n=60$ double bubble structures that can be constructed using the procedure discussed in Section 2. The cluster structures are relaxed so as to minimise the energy, which is initially defined using a semi-empirical potentials and then, during a final refinement stage, using density functional theory (DFT); see Sections 4.1 and 4.2 for details. The four double bubble clusters consist of: (a) only zinc oxide, denoted $(\mathrm{ZnO})_{12} @(\mathrm{ZnO})_{48}$; (b) only gallium nitride, denoted $(\mathrm{GaN})_{12} @(\mathrm{GaN})_{48} ;$ (c) a gallium nitride sodalite cage inside a zinc oxide bubble, denoted $(\mathrm{GaN})_{12} @(\mathrm{ZnO})_{48}$; and the inverse (d) a zinc oxide sodalite cage inside a gallium nitride bubble, denoted $(\mathrm{ZnO})_{12} @(\mathrm{GaN})_{48}$. During geometry optimization, although the high $T_{h}$ and $T$ symmetry that is maintained when semi-empirical calculations are employed, there is a reduction of symmetry for all four systems to $\mathrm{C}_{n}$, where $n=1$ or 2. As reported in Table 1, double bubble clusters with internal $(\mathrm{ZnO})_{12}$ sodalite cages adopt $\mathrm{C}_{2}$ symmetry, whereas those that had gallium nitride sodalite cages adopt $C_{1}$ symmetry-i.e., there is no symmetry in those structures. The average relaxed bond lengths, separated into inner-bubble bonds, outer-bubble bonds, and inter-layer bubble bonds ( $\mathrm{M}-\mathrm{X}$ bonds connecting the inner to the outer bubbles) are also reported in Table 1 . The average bond lengths of zinc oxide and gallium nitride are similar; although the average bond length for zinc oxide inner bubbles are slightly greater than the average bond lengths of gallium nitride inner bubbles.

Now, consider the distribution of bond lengths, $G(x)$, using a Gaussian broadening function for each bond length, which is normalised to the number of linkages between the inner and outer bubble ( $N=48$ for our $n=60$ double bubbles):

$$
G(x)=C \sum_{i=1}^{N} \exp \left(b_{i}-x\right)^{2} / 2 \sigma^{2}
$$

$C$ is a normalising constant, $b_{i}$ is the length of bond $i$, and $\sigma$ is the dispersion (width) of the Gaussian function. This function is plotted in Figure 5 for two values of $\sigma: 0.02 \AA$ (red line) and $0.10 \AA$ (blue line). The greater value of $\sigma$ allows the resolution of two distinct peaks for the systems of interest. These two peaks are reported in Table 1, labelled as $A$ and $B$ inter-bubble bond distances.

Table 1. Structural parameters of double bubble clusters, where $D_{\text {outer }}$ is the mean distance between $\mathrm{M}-\mathrm{X}$ atoms in the outer bubble, $D$ inner is the mean distance between $\mathrm{M}-\mathrm{X}$ atoms in the inner bubble, and $D_{\text {inter }}$ is the distance between the inner and outer bubbles. (Number in parentheses indicates standard error.)

\begin{tabular}{cccccc}
\hline \multirow{2}{*}{ System } & \multirow{2}{*}{ Symmetry } & $\boldsymbol{D}_{\text {outer }}(\boldsymbol{\AA})$ & $\boldsymbol{D}_{\text {inner }}(\AA)$ & \multicolumn{2}{c}{$\boldsymbol{D}_{\text {inter }}(\boldsymbol{\AA})$} \\
\cline { 5 - 6 } & & & & $\boldsymbol{A}$ & $\boldsymbol{B}$ \\
\hline$(\mathrm{GaN})_{12} @(\mathrm{ZnO})_{48}$ & $\mathrm{C} 1$ & 1.92 & 1.93 & $2.05(0.1)$ & $3.08(0.1)$ \\
$(\mathrm{ZnO})_{12} @(\mathrm{GaN})_{48}$ & $\mathrm{C} 2$ & 1.89 & 1.96 & $2.13(0.1)$ & $2.94(0.1)$ \\
$(\mathrm{ZnO})_{12} @(\mathrm{ZnO})_{48}$ & $\mathrm{C} 2$ & 1.93 & 1.95 & $2.10(0.0)$ & $2.94(0.2)$ \\
$(\mathrm{GaN})_{12} @(\mathrm{GaN})_{48}$ & $\mathrm{C} 1$ & 1.93 & 1.92 & $2.10(0.1)$ & $2.94(0.1)$ \\
\hline
\end{tabular}


Figure 5. Bond distribution plots for the double bubble cluster systems. Red line: Dispersion of Gaussian $=0.02$, Blue line: Dispersion of Gaussian $=0.1$.
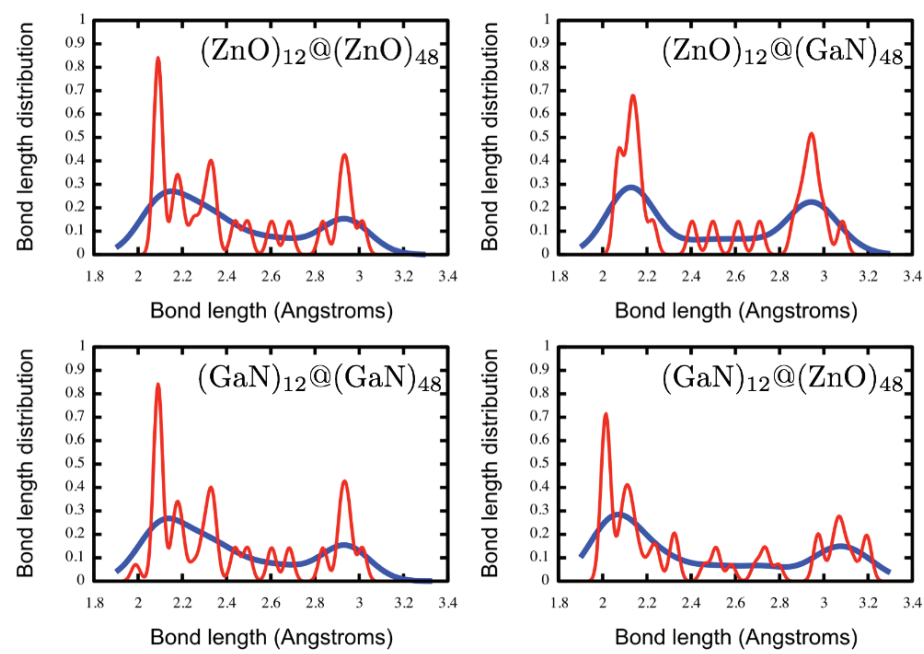

We observe that the pure double-bubble clusters have similar bond distributions, and notice only a difference of a small peak at $2 \AA$ for the pure GaN system, which appears as a shoulder on the $2.3 \AA$ peak in the pure $\mathrm{ZnO}$ system. We mark this shoulder (at approximately $2.25 \AA$ ) as the split of the distribution into bonded and non-bonded linkages. The number of bonded linkages, in fact, is constant for all the systems except for $(\mathrm{ZnO})_{12} @(\mathrm{GaN})_{48}$ and has a value of twenty-four, which is related to the ideal $\mathrm{T}$ symmetry octahedral shape. In this type of linking, two extremes can be possible: four of the eight hexagonal rings form drums with the outer bubble, or only half of the possible bonds are formed in such drums - see Figure 3b. The $(\mathrm{ZnO})_{12} @(\mathrm{GaN})_{48}$ double bubble, in contrast, has only twenty-two bonded linkages, which is not due to an inner bubble displacement from the centre of the outer bubble but is caused by a distortion in the outer bubble. To relate these observations to macroscopic properties of the systems, we considered the deformation as seen from the displacement of the centre of mass $(\mathrm{COM})$ of the inner bubbles with respect to the outer bubbles, and their normalized second moments of atom distribution, as given in Table 2.

Table 2. Centre of mass (COM) differences and normalised second moments of atom distributions for the double bubble clusters ( $x, y, z$ coordinates).

\begin{tabular}{cccc}
\hline \multirow{2}{*}{ System } & $\begin{array}{c}\text { COM difference } \\
\text { (COM }\end{array}$ & \multicolumn{2}{c}{ Normalised second moments of atom distribution } \\
\cline { 3 - 4 } & $0.00,0.11,0.05$ & $1.05,1.01,0.94$ & Outer \\
\hline$(\mathrm{GaN})_{12} @(\mathrm{ZnO})_{48}$ & $0.00,0.00,0.01$ & $1.02,1.00,0.98$ & $1.05,1.01,0.94$ \\
$(\mathrm{ZnO})_{12} @(\mathrm{GaN})_{48}$ & $0.00,0.00,0.04$ & $1.09,1.00,0.91$ & $1.01,1.00,0.99$ \\
$(\mathrm{ZnO})_{12} @(\mathrm{ZnO})_{48}$ & $0.00,0.01,-0.06$ & $1.04,1.01,0.96$ & $1.05,1.00,0.95$ \\
$(\mathrm{GaN})_{12} @(\mathrm{GaN})_{48}$ & 0
\end{tabular}

The largest COM displacement is seen in the $(\mathrm{GaN})_{12} @(\mathrm{ZnO})_{48}$ system and smallest in the inverse $(\mathrm{ZnO})_{12} @(\mathrm{GaN})_{48}$ system. The deformation is also lowest in the latter system, but has the largest 
values in pure $\mathrm{ZnO}$. We explain this behaviour by considering the relative sizes of the inner and outer bubbles: the larger $\mathrm{ZnO}$ inner bubble fills in the space offered by the smaller $\mathrm{GaN}$ outer bubble better than the GaN counterpart. An additional point to take into account is the greater flexibility of the $\mathrm{ZnO}$ bubbles as compared with $\mathrm{GaN}$ : the size mismatch between the inner and outer bubble is accommodated easier by $\mathrm{ZnO}$, the bubbles of which show the greater deformations. This flexibility is also seen in the bulk framework systems as discussed in Section 3.2 below. We show in Table 3 the energy of association, $E_{\text {Assoc, }}$, calculated as the difference in total energy of the double bubble cluster from their moieties, i.e., the $n_{1}=12$ and $n_{2}=48$ bubbles, and formation enthalpy, $H_{\mathrm{f}}$,

$$
H_{f}=\frac{E_{D B}-\left[n_{1}\left(E_{a}\right)+n_{2}\left(E_{b}\right)\right]}{n_{1}+n_{2}},
$$

where $E_{D B}$ is the total energy of the double bubble cluster, $E_{a}$ and $E_{b}$ are the total energies of the pure bulk wurtzite structures, where $a$ and $b$ can be $\mathrm{ZnO}$ or $\mathrm{GaN}$. We find that the formation of the double bubble systems is most favourable for the $(\mathrm{GaN})_{12} @(\mathrm{ZnO})_{48}$ system and least favourable for the inverse system, and that the pure double bubbles have equal formation energies.

Table 3. Energy of association, $E_{\text {Assoc }}$ of single-shell cages and enthalpy of formation, $H_{\mathrm{f}}$ per atom for double bubble clusters as defined in Equation (2).

\begin{tabular}{ccc}
\hline System & $\boldsymbol{E}_{\text {Assoc }}(\mathbf{k J} / \mathbf{m o l})$ & $\boldsymbol{H}_{\mathbf{f}}(\mathbf{k J} / \mathbf{m o l})$ \\
\hline$(\mathrm{GaN})_{12} @(\mathrm{ZnO})_{48}$ & -11.27 & 78.32 \\
$(\mathrm{ZnO})_{12} @(\mathrm{GaN})_{48}$ & -8.17 & 104.55 \\
$(\mathrm{ZnO})_{12} @(\mathrm{ZnO})_{48}$ & -9.38 & 68.50 \\
$(\mathrm{GaN})_{12} @(\mathrm{GaN})_{48}$ & -9.16 & 116.18 \\
\hline
\end{tabular}

We find that the formation of the homogeneous $(\mathrm{ZnO})_{12} @(\mathrm{ZnO})_{48}$ system is the most favourable closely followed by the heterogeneous $(\mathrm{GaN})_{12} @(\mathrm{ZnO})_{48}$ system compared to the homogeneous bulk wurtzite phases. Systems that have an outer-bubble of GaN are less likely to form when compared with bulk (at zero temperature). If we consider the mixing energies per atom:

$$
E_{\text {mix }}=\frac{E_{D B}-\left[0.8\left(E_{a}\right)+0.2\left(E_{b}\right)\right]}{120}
$$

where $E_{a}$ and $E_{b}$ are the energies of the pure double bubbles that make up the mixed system, we find that the energy of mixing for $(\mathrm{GaN})_{12} @(\mathrm{ZnO})_{48}$ and $(\mathrm{ZnO})_{12} @(\mathrm{GaN})_{48}$ are $0.07 \mathrm{~kJ} / \mathrm{mol}$ and $-0.96 \mathrm{~kJ} / \mathrm{mol}$ respectively.

\subsection{Double Bubble Frameworks}

We took the double bubble frameworks that were constructed using the procedure discussed in Section 2, and also corresponding systems of pure $\mathrm{ZnO}$ and $\mathrm{GaN}$, and optimised their geometry (see Section 4.2 for details). The structural analysis performed in Section 3.1 was repeated for these frameworks. The calculated average bond lengths are presented in Table 4, again separated into inner-bubble bonds, outer-bubble bonds and, inter-layer bubble bonds (bonds connecting the inner to the outer bubbles). The graphs of the corresponding bond-length distribution analysis can be seen 
in Figure 6. Table 4 also has two additional pieces of information - the lattice parameter and the bulk modulus, which are available for these extended crystalline frameworks. Similar to the double bubble clusters, we find that the bonds in the $\mathrm{ZnO}$ inner bubble are slightly larger than the equivalent $\mathrm{GaN}$ bonds. In the framework systems this has a noticeable effect on the bond distribution: when the inner bubble is composed of $\mathrm{ZnO}$, the bond length distribution is no longer bi-modal but has a single peak at $2.3 \AA$ (Figure 6), which, similar to the double bubble clusters, is due to the larger $\mathrm{ZnO}$ bubble occupying the space inside the outer bubble. In this case, however, as the outer bubble is in a framework, it is unable to deform to the same degree as the gas-phase cluster, and only a single peak forms in the bond length distribution.

We also see that the lattice parameters for the double bubble frameworks are, as expected, related to the composition of the outer bubble, and that when the outer bubble is composed of $\mathrm{ZnO}$ the lattice parameters are larger. Comparing the bulk moduli of the systems, we find that the pure GaN system is the least compressible, whereas the pure $\mathrm{ZnO}$ system is the most. This agrees with the double bubble cluster findings, where the $\mathrm{ZnO}$ systems exhibit the greatest deformations. Table 5 shows the corresponding structural parameters for the wurtzite systems used in the framework analysis, and the bulk modulus of the GaN system is much larger than that of the $\mathrm{ZnO}$.

Table 4. Structural parameters of double bubble frameworks. (Number in parentheses indicates standard error).

\begin{tabular}{|c|c|c|c|c|c|c|}
\hline \multirow{2}{*}{ System } & \multirow{2}{*}{$\begin{array}{c}\text { Lattice } \\
\text { parameter }(\AA)\end{array}$} & \multirow{2}{*}{$\begin{array}{c}\text { Bulk modulus } \\
(\mathrm{GPa})\end{array}$} & \multirow{2}{*}{$D_{\text {outer }}(\AA)$} & \multirow{2}{*}{$D_{\text {inner }}(\AA)$} & \multicolumn{2}{|c|}{$D_{\text {inter }}(\AA)$} \\
\hline & & & & & $A$ & $B$ \\
\hline$(\mathrm{GaN})_{12} @(\mathrm{ZnO})_{48}$ & 19.26 & 78.84 & 1.96 & 1.94 & $2.08(0.1)$ & $2.96(0.2)$ \\
\hline$(\mathrm{ZnO})_{12} @(\mathrm{GaN})_{48}$ & 18.84 & 77.88 & 1.90 & 2.01 & $2.27(0.1)$ & - \\
\hline$(\mathrm{ZnO})_{12} @(\mathrm{ZnO})_{48}$ & 19.26 & 69.78 & 1.94 & 2.00 & $2.26(0.0)$ & - \\
\hline$(\mathrm{GaN})_{12} @(\mathrm{GaN})_{48}$ & 18.94 & 101.92 & 1.93 & 1.94 & $2.18(0.2)$ & $3.04(0.1)$ \\
\hline
\end{tabular}

Figure 6. Bond distribution plots for the double bubble frameworks. Red line: Dispersion of Gaussian $=0.02$, Blue line: Dispersion of Gaussian $=0.1$.
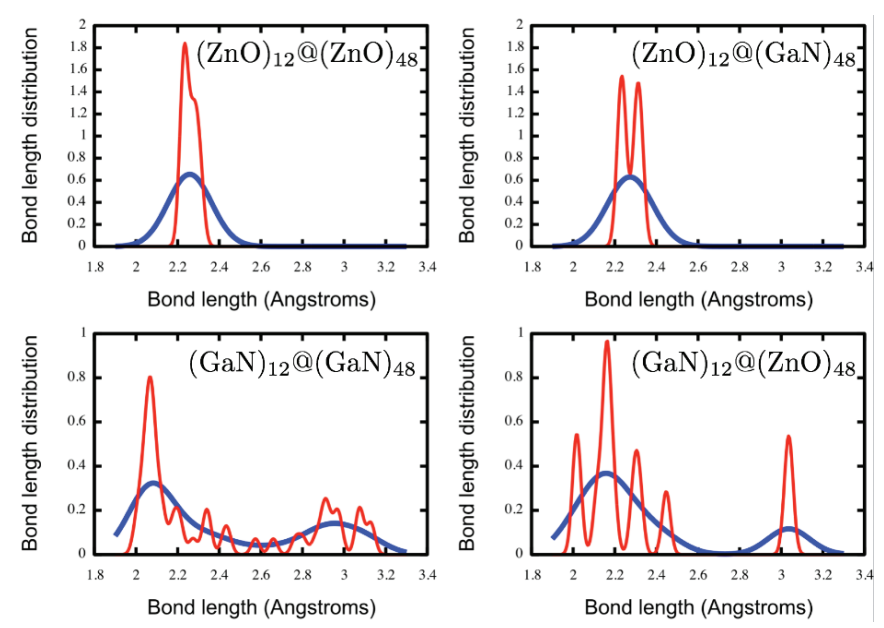
Table 5. Structural parameters of wurtzite phases.

\begin{tabular}{ccccc}
\hline System & Lattice parameter, $\boldsymbol{a}(\mathbf{\AA})$ & Lattice parameter, $\boldsymbol{c}(\boldsymbol{\AA})$ & Bulk modulus $(\mathbf{G P a})$ & $\boldsymbol{u}$ \\
\hline $\mathrm{ZnO}$ & 3.251 & 5.204 & 146.136 & 0.382 \\
$\mathrm{GaN}$ & 3.187 & 2.760 & 188.367 & 0.378 \\
\hline
\end{tabular}

We observe that the framework system of $(\mathrm{GaN})_{12} @(\mathrm{ZnO})_{48}$ has a similar inter-bond length distribution to that found in the double bubble systems which is again due to the fact that the smaller GaN cage has more freedom to move inside the larger $\mathrm{ZnO}$ bubble. The $(\mathrm{GaN})_{12} @(\mathrm{ZnO})_{48}$ system has a more clearly defined bi-modal distribution for the framework systems than observed for the double bubble cluster systems, and is likely due to reduced degrees of structural freedom with the extended bulk framework. Table 6 gives the formation enthalpies for the framework systems, and although these energies are positive i.e., unfavourable with respect to the pure bulk wurtzite phases, they are small enough to be accessible at experimental temperatures, and are comparable to the formation of fullerene $\left(\mathrm{C}_{60}\right)$ with respect to bulk carbon $(c a .40 \mathrm{~kJ} / \mathrm{mol})$ [25,26]. The pure GaN double bubble framework was found to be the least likely to form, whereas the $(\mathrm{GaN})_{12} @(\mathrm{ZnO})_{48}$ framework was found to be most favourable - again agreeing with the formation energy double bubble cluster findings.

Table 6. Enthalpy of formation per atom of double bubble frameworks as defined in Equation (2).

\begin{tabular}{cc}
\hline System & $\boldsymbol{H}_{\mathrm{F}} /$ atom $(\mathbf{k J} / \mathbf{m o l})$ \\
\hline$(\mathrm{GaN})_{12} @(\mathrm{ZnO})_{48}$ & 13.17 \\
$(\mathrm{ZnO})_{12} @(\mathrm{GaN})_{48}$ & 21.46 \\
$(\mathrm{ZnO})_{12} @(\mathrm{ZnO})_{48}$ & 18.54 \\
$(\mathrm{GaN})_{12} @(\mathrm{GaN})_{48}$ & 27.71 \\
\hline
\end{tabular}

When we compare the energies of mixing (using Equation (3)) we find that the energies per atom for $(\mathrm{GaN})_{12} @(\mathrm{ZnO})_{48}$ and $(\mathrm{ZnO})_{12} @(\mathrm{GaN})_{48}$ are $1.98 \mathrm{~kJ} / \mathrm{mol}$ and $-2.61 \mathrm{~kJ} / \mathrm{mol}$, respectively.

\section{Computational Detail}

\subsection{Interatomic Potentials Calculations}

We have used the semi-classical GULP code [27] to construct and optimise ZnO structures prior to refining them with DFT. We employed polarisable shell inter-atomic potentials parameterised for bulk $\mathrm{ZnO}[7,28]$ in the double bubble cluster and framework calculations. The resulting atomic structures were used not only for $\mathrm{ZnO}$, but also $\mathrm{GaN}$ and mixed $\mathrm{ZnO} / \mathrm{GaN}$ structures; note that the bond lengths in $\mathrm{GaN}$ are very similar those in $\mathrm{ZnO}$ (see Tables 1-3 in Section 3), and we only required approximate initial atomic coordinates for input into the DFT calculations, as outlined below. 


\subsection{Density Functional Theory Calculations}

In all of the $a b$ initio calculations, we have used the solids-corrected Perdew-Burke-Ernzerhof (PBEsol) GGA exchange-correlation functional [29,30], and all structural optimisations were deemed converged when the atomic forces were less than $0.01 \mathrm{eV} / \AA$.

A natural choice for the calculations on the double bubble clusters, due to its computational efficiency, is the DFT code FHI-aims [31] as it uses numeric atom-centred basis sets. These calculations were performed with the species defaults for the "tight" basis sets for accuracy (energies converged to $1 \mathrm{meV} /$ atom) and with scalar ZORA relativistic treatment [32]. We have used the plane-wave DFT code VASP [33-36] to determined the equilibrium structures of the double bubble based framework (extended crystal systems - see Section 2), and, for comparison, wurtzite bulk ZnO and GaN. Within VASP, we employed the projector augmented wave (PAW) method [37] to describe the interactions between the cores $(\mathrm{Zn}:[\mathrm{Ar}], \mathrm{Ga}:[\mathrm{Ar}], \mathrm{O}:[\mathrm{He}]$ and $\mathrm{N}:[\mathrm{He}])$ and the valence electrons.

To determine the equilibrium bulk structures avoiding the problem of Pulay stress, we have optimised the atomic coordinates at a series of different volumes, and fitted the resulting energy versus volume data to the Murnaghan equation of state.

We have found that for the framework systems, an energy cut-off of $500 \mathrm{eV}$, and Monkhorst-Pack $k$-point meshes of $8 \times 8 \times 6$ and $1 \times 1 \times 1$ for, respectively, the pure bulk wurtzite systems, and

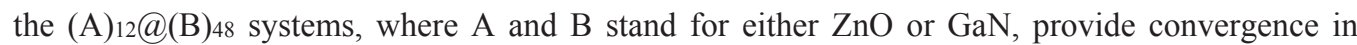
total energy up to $10^{-5} \mathrm{eV}$ for the framework systems, which is comparable with our double bubble cluster calculations.

\section{Conclusions}

We have constructed double-bubble clusters and frameworks of $\mathrm{ZnO}$ and $\mathrm{GaN}$ from a bottom up approach from cage structures analogous to fullerenes formed from hexagonal building units $[15,16,22]$. The four systems we have considered, $(\mathrm{GaN})_{12} @(\mathrm{ZnO})_{48},(\mathrm{ZnO})_{12} @(\mathrm{GaN})_{48}$, $(\mathrm{ZnO})_{12} @(\mathrm{ZnO})_{48}$ and $(\mathrm{GaN})_{12} @(\mathrm{GaN})_{48}$, were first geometry optimized using a semi-empirical potential within the GULP code and then refined using FHI-aims (for the double bubble clusters) or VASP (for the frameworks) at the DFT level of theory using the PBEsol exchange-correlation functional. We found that although the average bond lengths of both $\mathrm{ZnO}$ and $\mathrm{GaN}$ are similar, the average bond lengths for $\mathrm{ZnO}$ inner bubbles were larger than the $\mathrm{GaN}$ inner bubbles of both the double bubble cluster systems and the frameworks. This relative size difference, we believe, means that the larger $\mathrm{ZnO}$ inner bubble fills in the space offered by the smaller GaN outer bubble better than the GaN counterpart. In addition, we found that the greater flexibility of the $\mathrm{ZnO}$ bubbles from calculations of bulk moduli, as compared with that of GaN bubbles, means that the size mismatch between the inner bubble and outer bubble is more readily accommodated by $\mathrm{ZnO}$. Furthermore, the structural analysis of the pure $\mathrm{ZnO}$ double bubbles also showed the greater deformations. The average $\mathrm{M}-\mathrm{X}$ inter-bubble bonds were found to exhibit a bi-modal distribution for both clusters and frameworks, except for the pure $\mathrm{ZnO}$ and $(\mathrm{ZnO})_{12} @(\mathrm{GaN})_{48}$ framework systems. These single-peak distributions were due to the larger $\mathrm{ZnO}$ inner bubble that has less freedom to move than in the inverse systems. The association energies of the double bubble clusters show that the systems 
investigated here are favourable when compared to individual bubbles, although when compared to bulk wurtzite phases, the clusters are less favourable.

The standard formation enthalpies for the framework systems are lower than those of carbon fullerenes. Therefore, we suggest that these double bubble systems should be thermodynamically accessible and could provide valuable material properties in the future.

\section{Acknowledgments}

We thank kindly our former collaborators Said Hamad, Eleonora Spano, Stefan T. Bromley, Stephen A. Shevlin, Matthew B. Watkins, and Abdullah A. Al-Sunaidi, discussions with who have been inspiring and instrumental for us undertaking this research. We also thank EPSRC for providing the funding for Matthew Farrow and Scott Woodley on grant numbers EP/I03014X9 and EP/K038958; John Buckeridge and Alexey Sokol on grant number EP/IO1330X; and Andrew Logsdail on grant numbers EP/I030662/1 and EP/K038419/1. The authors also acknowledge the use of the UCL Legion High Performance Computing Facility (Legion@UCL) and associated support services; the IRIDIS cluster provided by the EPSRC funded Centre for Innovation (EP/K000144 and EP/K000136); this work made use of the facilities of HECToR and ARCHER, the UK's national high-performance computing service through membership of the UK's HPC Materials Chemistry Consortium, which is funded by EPSRC (EP/L000202).

\section{Author Contributions}

The structures were constructed by Scott M. Woodley. The interatomic potential calculations were performed by Scott M. Woodley and Alexey A. Sokol. The double bubble cluster calculations were performed by Matthew R. Farrow. The double bubble framework calculations were performed by John Buckeridge. The literature was researched by Andrew J. Logsdail and David O. Scanlon. Expertise both in relevant materials science and methodology was provided by C. Richard A. Catlow and Scott M. Woodley. Vital contributions to simulations design, the data analysis and preparation of the manuscript were made by all of the authors.

\section{Conflicts of Interest}

The authors declare no conflict of interest.

\section{References}

1. Fujishima, A.; Honda, K. Electrochemical photolysis of water at a semiconductor electrode. Nature 1972, 238, 37-38.

2. Vispute, R.D.; Talyansky, V.; Choopun, S.; Sharma, R.P.; Venkatesan, T.; He, M.; Tang, X.; Halpern, J.B.; Spencer, M.G.; Li, Y.X.; et al. Heteroepitaxy of $\mathrm{ZnO}$ on $\mathrm{GaN}$ and its implications for fabrication of hybrid optoelectronic devices. Appl. Phys. Lett. 1998, 73, 348-350.

3. Nakamura, S.; Pearton, S.; Fasol, G. The Blue Laser Diode: The Complete Story; Springer-Verlag: Berlin, Germany, 2000. 
4. Yu, Q.-X.; Xu, B.; Wu, Q.-H.; Liao, Y.; Wang, G.-Z.; Fang, R.-C.; Lee, H.-Y.; Lee, C.-T. Optical properties of $\mathrm{ZnO} / \mathrm{GaN}$ heterostructure and its near-ultraviolet light-emitting diode. Appl. Phys. Lett. 2003, 83, 4713.

5. Zhu, H.; Shan, C.-X.; Yao, B.; Li, B.-H.; Zhang, J.-Y.; Zhang, Z.-Z.; Zhao, D.-X.; Shen, D.-Z.; Fan, X.-W.; Lu, Y.-M.; et al. Ultralow-threshold laser realized in zinc oxide. Adv. Mater. 2009, 21, 1613-1617.

6. Shevlin, S.A.; Guo, Z.X.; van Dam, H.J.J.; Sherwood, P.; Catlow, C.R.A.; Sokol, A.A.; Woodley, S.M. Structure, optical properties and defects in nitride (III-V) nanoscale cage clusters. Phys. Chem. Chem. Phys. 2008, 10, 1944-1959.

7. Catlow, C.R.A.; French, S.A.; Sokol, A.A.; Al-Sunaidi, A.A.; Woodley, S.M. Zinc oxide: A case study in contemporary computational solid state chemistry. J. Comput. Chem. 2008, 29, 2234-2249.

8. Catlow, C.R.A.; Bromley, S.T.; Hamad, S.; Mora-Fonz, M.; Sokol, A.A.; Woodley, S.M. Modelling nano-clusters and nucleation. Phys. Chem. Chem. Phys. 2010, 12, 786-811.

9. Watkins, M.B.; Shevlin, S.A.; Sokol, A.A.; Slater, B.; Catlow, C.R.A.; Woodley, S.M. Bubbles and microporous frameworks of silicon carbide. Phys. Chem. Chem. Phys. 2009, 11, 3186-3200.

10. Woodley, S.M.; Watkins, M.B.; Sokol, A.A.; Shevlin, S.A.; Catlow, C.R.A. Construction of nano- and microporous frameworks from octahedral bubble clusters. Phys. Chem. Chem. Phys. 2009, 11, 3176-3185.

11. Carrasco, J.; Illas, F.; Bromley, S.T. Ultralow-density nanocage-based metal-oxide polymorphs. Phys. Rev. Lett. 2007, 99, 235502.

12. Hamad, S.; Catlow, C.R.A.; Spanó, E.; Matxain, J.M.; Ugalde, J.M. Structure and properties of ZnS nanoclusters. J. Phys. Chem. B 2005, 109, 2703-2709.

13. Al-Sunaidi, A.A.; Sokol, A.A.; Catlow, C.R.A.; Woodley, S.M. Structures of zinc oxide nanoclusters: As found by revolutionary algorithm techniques. J. Phys. Chem. C 2008, 112, 18860-18875.

14. Behrman, E.C.; Foehrweiser, R.K.; Myers, J.R.; French, B.R.; Zandler, M.E. Possibility of stable spheroid molecules of ZnO. Phys. Rev. A 1994, 49, R1543-R1546.

15. Jensen, F.; Toftlund, H. Structure and stability of C24 and B12N12 isomers. Chem. Phys. Lett. 1993, 201, 89-96.

16. Golberg, D.; Bando, Y.; Stephan, O.; Kurashima, K. Octahedral boron nitride fullerenes formed by electron beam irradiation. Appl. Phys. Lett. 1998, 73, 2441-2443.

17. Woodley, S.M. Applications of Evolutionary Computation in Chemistry; Springer: Berlin, Germany, 2004; Volume 110.

18. Woodley, S.M. Prediction of crystal structures using evolutionary algorithms and related techniques. In Applications of Evolutionary Computation in Chemistry; Springer-Verlag: Berlin, Germany, 2004; Volume 110, pp. 95-132.

19. Foster, M.D.; Friedrichs, O.D.; Bell, R.G.; Paz, F.A.A.; Klinowski, J. Structural evaluation of systematically enumerated hypothetical uninodal zeolites. Angew. Chem. 2003, 115, 4026-4029. 
20. Zwijnenburg, M.A.; Illas, F.; Bromley, S.T. Apparent scarcity of low-density polymorphs of inorganic solids. Phys. Rev. Lett. 2010, 104, 175503.

21. Enyashin, A.N.; Gemming, S.; Bar-Sadan, M.; Popovitz-Biro, R.; Hong, S.Y.; Prior, Y.; Tenne, R.; Seifert, G. Structure and stability of molybdenum sulfide fullerenes. Angew. Chem. Int. Ed. 2007, 46, 623-627.

22. Parilla, P.A.; Dillon, A.C.; Jones, K.M.; Riker, G.; Schulz, D.L.; Ginley, D.S.; Heben, M.J. The first true inorganic fullerenes? Nature 1999, 397, 114.

23. Kasuya, A.; Sivamohan, R.; Barnakov, Y.A.; Dmitruk, I.M.; Nirasawa, T.; Romanyuk, V.R.; Kumar, V.; Mamykin, S.V.; Tohji, K.; Jeyadevan, B.; et al. Ultra-stable nanoparticles of CdSe revealed from mass spectrometry. Nat. Mater. 2004, 3, 99-102.

24. Botti, S.; Marques, M.A.L. Identification of fullerene-like cdse nanoparticles from optical spectroscopy calculations. Phys. Rev. B 2007, 75, 035311.

25. Diogo, H.P.; da Piedade, M.E.M.; Dennis, T.J.S.; Hare, J.P.; Kroto, H.W.; Taylor, R.; Walton, D.R.M. Enthalpies of formation of buckminsterfullerene $\left(\mathrm{C}_{60}\right)$ and of the parent ions $\mathrm{C}_{60}{ }^{+}, \mathrm{C}_{60}{ }^{2+}, \mathrm{C}_{60}{ }^{3+}$ and $\mathrm{C}_{60}{ }^{-}$. J. Chem. Soc. Faraday Trans. 1993, 89, 3541-3544.

26. Curl, R.F.; Haddon, R.C. On the formation of the fullerenes. Philos. Trans. 1993, 343, 19-32.

27. Gale, J.D.; Rohl, A.L. The general utility lattice program (gulp). Mol. Simul. 2003, 29, 291-341.

28. Whitmore, L.; Sokol, A.A.; Catlow, C.R.A. Surface structure of zinc oxide (1010), using an atomistic, semi-infinite treatment. Surf. Sci. 2002, 498, 135-146.

29. Perdew, J.P.; Burke, K.; Ernzerhof, M. Generalized gradient approximation made simple. Phys. Rev. Lett. 1996, 77, 3865-3868.

30. Perdew, J.P.; Ruzsinszky, A.; Csonka, G.I.; Vydrov, O.A.; Scuseria, G.E.; Constantin, L.A.; Zhou, X.; Burke, K. Restoring the density-gradient expansion for exchange in solids and surfaces. Phys. Rev. Lett. 2008, 100, 136406.

31. Blum, V.; Gehrke, R.; Hanke, F.; Havu, P.; Havu, V.; Ren, X.; Reuter, K.; Scheffler, M. Ab initio molecular simulations with numeric atom-centered orbitals. Comput. Phys. Commun. 2009, 180, 2175-2196.

32. Van Lenthe, E.; Baerends, E.J.; Snijders, J.G. Relativistic total energy using regular approximations. J. Chem. Phys. 1994, 101, 9783.

33. Kresse, G.; Hafner, J. Ab initio molecular dynamics for liquid metals. Phys. Rev. B 1993, 47, $558-561$.

34. Kresse, G.; Hafner, J. Ab initio molecular-dynamics simulation of the liquid-metal-amorphoussemiconductor transition in germanium. Phys. Rev. B 1994, 49, 14251-14269.

35. Kresse, G.; Furthmüller, J. Efficiency of ab-initio total energy calculations for metals and semiconductors using a plane-wave basis set. Comput. Mater. Sci. 1996, 6, 15-50.

36. Kresse, G.; Furthmüller, J. Efficient iterative schemes for $a b$ initio total-energy calculations using a plane-wave basis set. Phys. Rev. B 1996, 54, 11169-11186.

37. Blöchl, P.E. Projector augmented-wave method. Phys. Rev. B 1994, 50, 17953-17979. 


\title{
Thermoplastic Polymer Nanocomposites Based on Inorganic Fullerene-like Nanoparticles and Inorganic Nanotubes
}

\section{Mohammed Naffakh and Ana M. Díez-Pascual}

\begin{abstract}
Using inorganic fullerene-like (IF) nanoparticles and inorganic nanotubes (INT) in organic-inorganic hybrid composite, materials provide the potential for improving thermal, mechanical, and tribological properties of conventional composites. The processing of such high-performance hybrid thermoplastic polymer nanocomposites is achieved via melt-blending without the aid of any modifier or compatibilizing agent. The incorporation of small quantities $(0.1-4 \mathrm{wt} . \%)$ of IF/INTs (tungsten disulfide, IF-WS 2 or molybdenum disulfide, $\mathrm{MoS}_{2}$ ) generates notable performance enhancements through reinforcement effects and excellent lubricating ability in comparison with promising carbon nanotubes or other inorganic nanoscale fillers. It was shown that these IF/INT nanocomposites can provide an effective balance between performance, cost effectiveness, and processability, which is of significant importance for extending the practical applications of diverse hierarchical thermoplastic-based composites.
\end{abstract}

Reprinted from Inorganics. Cite as: Naffakh, M.; Díez-Pascual, A.M. Thermoplastic Polymer Nanocomposites Based on Inorganic Fullerene-like Nanoparticles and Inorganic Nanotubes. Inorganics 2014, 2, 291-312.

\section{Introduction}

Over the past few years, research interest in the field of thermoplastic composites has changed from "high-performance" advanced materials towards the development of "cost-performance" engineering composites. Especially, carbon fiber (CF) or glass fiber (GF) reinforced, thermoplastic-based composites have shown to offer design, processing, performance, and cost advantages compared to metals for manufacturing structural parts. Among the advantages provided by fiber-reinforced thermoplastics over metals and ceramics, that have been recognized for years, are improved fracture toughness, impact resistance, strength to weight ratio, as well as high resistance to corrosion and enhanced thermal and fatigue properties that have often been put in good use for practical applications in the aeronautic, automotive, and energy sectors [1-3]. Nevertheless, these applications require new properties and functionalities, especially superior mechanical performance, flame and chemical resistance, magnetic field and UV resistance, high electrical conductivity, environmental stability, low water absorption, and so forth. To address these issues, the integration of inorganic nanoparticles into a polymer matrix allows both properties from inorganic nanoparticles and polymer to be combined, thus, resulting in advanced polymer nanocomposites (PNCs) [4]. In particular, additional nanoscale fillers, such as carbon nanotubes (CNTs) [5] or inorganic nanoparticles [6], have been mixed with CFs to reinforce polymer matrices. Their high specific surface area enables the formation of a large interphase in the composite and strong filler-matrix interactions. In the same way, the addition of nanoclays to fiber-reinforced thermoplastic composites has been reported to improve damping properties, fatigue life, toughness, and wear resistance $[7,8]$. 
The synergetic effect of CFs with the inorganic nanoparticles is believed to be the major cause for the mechanical improvement achieved.

Recently, inorganic fullerenes (IFs) and nanotubes (INTs), based on layered metal dichalcogenides, such as $\mathrm{WS}_{2}$ and $\mathrm{MoS}_{2}$, have emerged as one of the most promising developments in the area of nanomaterials. These types of nanoparticles are currently the subject of intense research, summarized in these reviews that include synthetic methodologies, diverse properties of these new nanomaterials and their potential applications [9,10]. The first synthesis of such nanoparticles was reported by Tenne et al., in 1992 and 1993 [11,12]. Since then, the synthetic technology has advanced considerably and almost pure materials ( $>99 \%$ ) are currently synthesized in large amounts by ApNano Materials, Inc. (NanoMaterials, Ltd., Yavne, Israel) and employed in a wide variety of fields, such as aerospace, automotive, naval, defense, medical, energy, electronics, and various other industries. The physical properties of $\mathrm{WS}_{2}$ and $\mathrm{MoS}_{2}$ nanostructures (IF/INTs) have been studied in detail, both experimentally and by theoretical modeling. These properties are interesting, not only academically, but also because these kinds of nanostructures show substantial potential for becoming part of the ultrahigh-strength nanocomposite technology [13].

The objective of this article is to emphasize the most recent findings about the influence of IF nanoparticles and INTs on the structure, morphology and properties of thermoplastic polymer nanocomposites, in comparison with PNCs incorporating other nanofillers. Particular interest has been devoted to analyze the thermal, mechanical, and tribolological property enhancements attained in multiscale fiber-reinforced thermoplastic composites containing inorganic fullerene-like $\mathrm{WS}_{2}$ nanoparticles.

\section{Preparation and Dispersion of IF/INT into Thermoplastic Polymers}

The mixing of polymers and nanoparticles is opening new avenues of research and development of advanced engineering flexible composites that exhibit advantageous magnetic, electrical, optical, or mechanical properties. The main challenge in fabrication of these polymer nanocomposites for structural and functional applications is uniform dispersion of nanoparticles in the polymer matrix. However, good dispersion of nanoparticles in polymer composite materials is extremely difficult to achieve since nanoparticles have a strong tendency to aggregate due to their nano-size and high surface energy. In the case of organic-inorganic nanocomposites, the strength or level of interaction between the organic and inorganic phases is another important factor in improving the overall properties of the composites. Physical or simple mechanical mixing usually lead to a weak interaction between the phases via hydrogen bonding or van der Waals forces. In order to minimize interface energies between particles and polymer matrices, several surface modification/functionalization and stabilization techniques have been developed that are mainly used in chemical methods, such as sol-gel, in situ polymerization, etc. Owing to numerous papers published on polymer organic-inorganic composite materials, it is impossible to completely review this field. The reader is referred to the literature cited for a more detailed description of synthetic methods used for the processing of PNCs reinforced with different types of inorganic nanofillers [13-15]. 
Inorganic layered materials, such as transition metal dichalcogenides $\mathrm{MS}_{2}(\mathrm{M}=\mathrm{Mo}, \mathrm{W})$, are one of the most modern and the most promising development areas in the field of nanomaterials. Inorganic fullerene-like (IF) nanoparticles can provide significant advantages over other spherical nanoparticles for the preparation of advanced PNCs [13]. In particular, the incorporation of environmentally-friendly IF-WS 2 nanoparticles has been shown to improve thermal, mechanical, and tribological properties of a series of thermoplastic polymers, including isotactic polypropylene (iPP) [16], polyphenylene sulfide (PPS) [17], poly(ether ether ketone) (PEEK) [18], and nylon-6 [19]. The efficient dispersion of IF-WS 2 was achieved through simple melt-blending without using modifiers or surfactants. Moreover, the combination of inorganic fullerenes with other organic micro-particles (nucleating agents), micro-fibers (CFs) or nanofillers (CNTs) allows tailoring of more sophisticated hybrid materials with complex architectures, interactions, morphology, and functionality [20-24]. In the same way, the use of INT-WS $2\left(\mathrm{MoS}_{2}\right)$ offers the opportunity to produce novel advanced polymer nanocomposite materials with excellent nanoparticle dispersion. More specifically, since the beginning of 2011, we have successfully developed a new family of nanocomposites, which integrated $\mathrm{MoS}_{2}$ nanotubes into an isotactic polypropylene (iPP) matrix, one of the most widely investigated polymers in the preparation and application of nanocomposites, employing a simple and cost effective melt-processing route [25]. This strategy yields finer dispersion, with INT-MoS 2 almost fully debundled into individual tubes or small clusters, which are randomly oriented in the iPP matrix. Additionally, well-dispersed $\mathrm{WS}_{2}$ inorganic nanotubes were efficiently incorporated into epoxy matrix, poly(methyl methacrylate) (PMMA), poly(propylene fumarate) (PPF), and poly(3-hydroxybutyrate) (PHB), using various processing techniques [26-29]. Figure 1 shows, as an example, typical SEM images of the fracture surfaces of composites containing inorganic fullerene-like nanoparticles or inorganic nanotubes obtained under optimal processing conditions. It has been demonstrated by statistical analysis of the surface density of IF-WS 2 nanoparticles in the iPP nanocomposites, that the degree of dispersion strongly depends on the duration of melt blending [16]. For 1.0 wt.\% IF-WS 2 (Figure 1a), it can be seen that these nanoparticles are almost spherical, with an average diameter of around $80 \mathrm{~nm}$, similar to that observed for the raw nanofiller, and are individually dispersed for mixing times between 5 and $20 \mathrm{~min}$. However, for IF-WS 2 contents $\geq 4.0 \mathrm{wt} . \%, 5 \mathrm{~min}$ is not enough time to attain single particle distribution, and for the highest concentration incorporated of $8.0 \mathrm{wt} \%$ (not shown here), the influence of the mixing time on the degree of dispersion is even stronger. With increasing loading, the interparticle distance decreases, hence, flocculation of these nanoparticles can occur after the mixing is stopped. Thus, the crystallization rate, as well as the modulus of iPP, initially rise with increasing filler content and finally level-off at filler loadings of around 1.0 wt.\% [16]. In the case of multiscale fiber-reinforced thermoplastic composites, the laminates were prepared by the film-stacking process. Four layers of GF or CF were alternatively stacked within five iPP/IF-WS 2 (PPS/IF-WS 2 ) films in a closed mold. Consolidation of the material was made at $210^{\circ} \mathrm{C}$ in a hot-press $\left(320^{\circ} \mathrm{C}\right.$ in the case of PPS matrix) [22,23]. The results obtained are very promising and suggest that the use of IF/INT can provide an effective balance between cost effectiveness and processability, making the resulting polymer nanocomposites highly suitable for a wide range of applications at a large scale. 
Figure 1. SEM micrographs of novel polymer/IF(INT) nanocomposites. (a) iPP/IF-WS (1.0 wt.\%); (b) PPS/IF-WS 2 (1.0 wt.\%); (c) iPP/INT-MoS2 (1.0 wt.\%); (d) iPP/IF-WS (2.0 wt.\%)/GF and (e) PPS/IF-WS $2(2.0$ wt. \%)/CF.
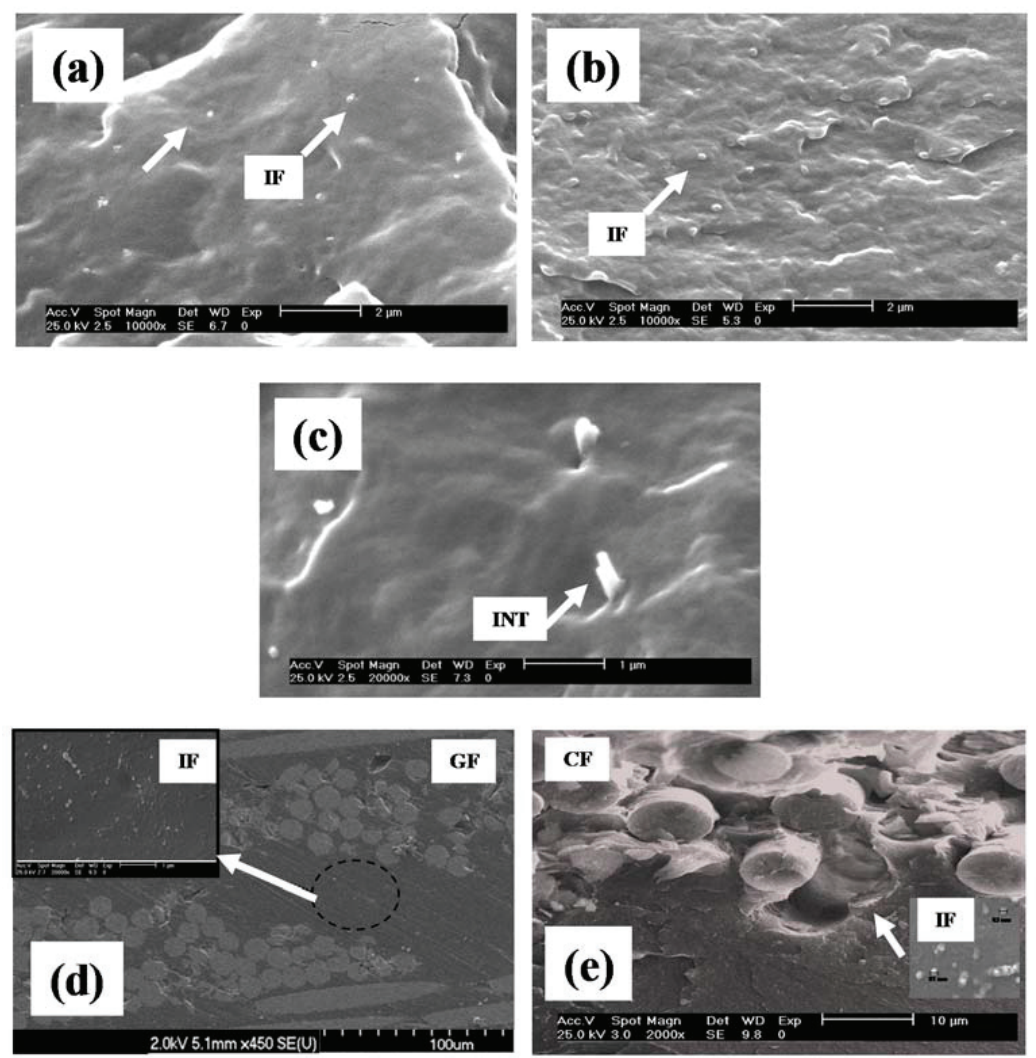

\section{Thermal Properties}

It is well known that the crystalline morphology and structure obtained during the thermoplastic processing plays an important role on the physico-mechanical behavior of the resulting polymeric material, conditioning its potential uses. In this way, the control of the crystallization process can be seen as a successful approach for improving physico-mechanical properties of polymers. Therefore, it is of great interest to investigate the nucleation, crystallization, and structural development of the matrix in IF/INT reinforced polymer nanocomposites [13]. This would help to optimize the manufacturing conditions in order to obtain high-performance nanocomposites and to fully exploit their potential in practical applications. 
Figure 2. TGA thermograms under a nitrogen atmosphere for neat iPP, PPS and some hierarchical laminates. The inset shows the initial degradation temperature $\left(T_{\mathrm{i}}\right)$ vs. nanoparticle loading.

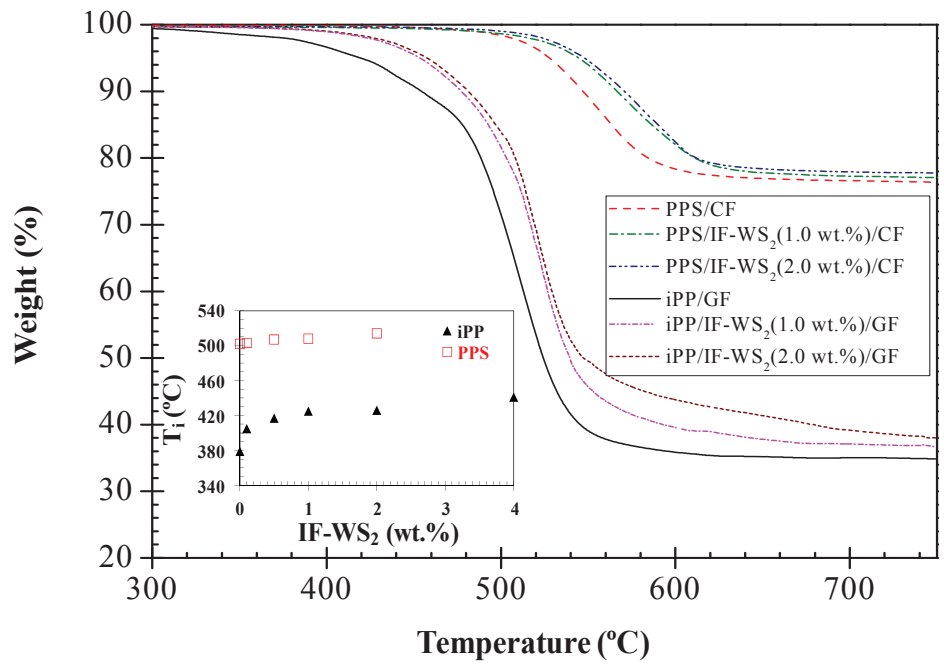

The thermal stability of several polymer matrices reinforced with IF-WS 2 nanoparticles was compared with that observed for other spherical inorganic nanofillers, organized by the nature of the matrix [13]. It was found that the incorporation of nanometer-sized $\mathrm{k}$ particles into a polymer enhances the thermal stability of the matrix inhibiting the formation and escape of volatile byproducts generated during the decomposition process. In the case of the hierarchical thermoplastic-based composites, the thermal stability of IF-WS 2 reinforced iPP [22] and PPS [23] laminates has been investigated using TGA, and typical thermograms under a nitrogen atmosphere for the neat matrices, and composites reinforced with 1.0 and $2.0 \mathrm{wt} . \% \mathrm{IF}_{-} \mathrm{WS}_{2}$ are shown in Figure 2. It is found that all the composites exhibit a single decomposition stage in a nitrogen environment, similar to that found for the neat polymers, indicating that the random scission of the polymeric chains is the predominant degradation process. The incorporation of increasing nanoparticle contents induces a progressive thermal stabilization of both matrices (see inset of Figure 2), the effect being more significant in the case of iPP, probably related to the lower thermal stability of this commodity plastic compared to high-performance PPS. Thus, an increase in the initial degradation temperature $\left(T_{\mathrm{i}}\right)$ of $12{ }^{\circ} \mathrm{C}$ and $47^{\circ} \mathrm{C}$ is attained at $2.0 \mathrm{wt} . \%$ loading in comparison to the reference PPS and iPP laminate, respectively. A similar trend is found for the temperature of $10 \%$ weight loss $\left(T_{10}\right)$ and maximum rate of weight loss ( $\left.T_{\max }\right)$. This thermal stability enhancement has been ascribed to the barrier effect of the nanoparticles that effectively obstruct the diffusion of volatile products from the bulk of the polymer to the gas phase, therefore slowing down the decomposition process. Upon increasing IF-WS 2 loading, the barrier effect becomes stronger, which is reflected in higher degradation temperatures. An analogous effect of thermal stability increase has been reported for PP/GF composites reinforced with other inorganic nanoparticles such as clays [30]. Nevertheless, for the same nanofiller loading, the improvements in thermal stability are larger in the case of IF-WS 2 , indicative of a more effective 
heat barrier effect of the IF nanoparticles likely arising from their more homogenous dispersion and spherical shape, thus, larger specific surface area.

In the same way, the incorporation of INTs can also lead to an improvement in the thermal stability of polymer/INTs [25,27]. As an example, the characteristic weight loss temperatures for PP nanocomposites, filled with different nanoreinforcements in nitrogen, are summarized in Table 1 [31-42]. The data reveal that the concentration of non-modified INT-MoS 2 has a dramatic effect on the thermal stability of the iPP nanocomposites. $T_{10}$ of iPP/INT-MoS 2 (1.0 wt.\%) was almost $60{ }^{\circ} \mathrm{C}$ higher than that of neat iPP, suggesting that INT-MoS 2 have outstanding properties for improving the thermal stability at low nanofiller content [31]. As a comparison, approximately the same increment was observed for iPP nanocomposites filled with $10 \mathrm{wt} \%$ of silane-modified halloysite nanotubes (HNTs). In the case of iPP/HNTs, the thermal stability and flame-retardant effects are believed to result from the hollow tubular structure of HNTs, the barriers for heat and mass transport and the presence of iron in the HNTs [32-34]. Layered silicates, such as montmorillonite (MMT), also have important effects on the thermal stability of the PP matrix (Table 1). The dramatic improvement in thermal stability of around $90{ }^{\circ} \mathrm{C}$ was related to the confinement of the single nanoparticles in approximately $1 \mathrm{~nm}^{3}$ volume using sophisticated methods of modification/exfoliation [39-41].

The flammability behavior of PPS/IF-WS $/$ CF has been investigated by pyrolysis combustion flow calorimetry, in order to determine the heat release rate (HRR) at different nanoparticle contents [24]. The addition of IF-WS 2 leads to a progressive drop in the average peak HRR, the reduction being about $17 \%$ for the laminate with $1.0 \mathrm{wt} . \%$ loading. Further, the onset temperature at which begins the release of heat and the temperature at peak HRR increase gradually with the nanoparticle loading, with maximum increments of 19 and $23{ }^{\circ} \mathrm{C}$, respectively, at $2.0 \mathrm{wt} . \% \mathrm{IF}-\mathrm{WS}$. These improvements are probably related to the low degree of porosity and enhanced thermal stability of the hybrids. Moreover, there seems to be a synergistic effect of both micro- and nano-fillers on increasing the polymer resistance to fire. The coexistence of CFs and IF-WS 2 in the laminates results in a more effective confined geometry that increases the barrier resistance to the evolution of flammable volatiles. Similar synergistic behavior has been described for different polymer/clay/carbon nanotube hybrids $[43,44]$.

The degree of crystallinity is a key parameter in thermoplastic polymers because it has strong influence on both the chemical and mechanical properties. The crystalline phase improves the stiffness and tensile strength whilst the amorphous phase helps to absorb the impact energy. The influence of IF-WS $\mathrm{WS}_{2}$ on the crystallization behavior of PPS/CF [23] and iPP/GF [22] has been analyzed by DSC, and typical cooling thermograms for composites with 1.0 and $2.0 \mathrm{wt} . \%$ loading are shown in Figure 3. Moreover, the crystallization temperature $\left(T_{\mathrm{p}}\right)$ as a function of IF-WS2 concentration is plotted in the inset of this Figure. Noticeable differences are detected depending on the thermoplastic polymer. In the case of PPS based composites, the addition of low nanoparticle contents (i.e., 0.1 or $0.5 \mathrm{wt} . \%$ ) results in a decrease in $T_{p}$ and the degree of crystallinity $\left(X_{c}\right)$, indicating the absence of a nucleating effect of the IF-WS 2 on the polymer crystallization, and that the transport of macromolecular segments to the growing surface of PPS in the composite is hindered. However, the incorporation of higher nanoparticle contents leads to an increase in both $T_{p}$ and $X_{c}$, by up to 
$9{ }^{\circ} \mathrm{C}$ and $14 \%$, respectively demonstrating that higher nanoparticle contents act as nucleating agents for PPS. On the other hand, these nanoparticles effectively nucleate the iPP matrix in the concentration range of $0-4.0 \mathrm{wt} . \%$, with increases up to $22{ }^{\circ} \mathrm{C}$ and $6 \%$ in $T_{p}$ and $X_{\mathrm{c}}$, respectively, at the highest loading tested. These improvements are greater than those reported for binary $\mathrm{iPP} / \mathrm{IF}-\mathrm{WS}_{2}$ nanocomposites [16], pointing towards a synergistic effect of both fillers on promoting the crystallization of iPP. This behavior is in agreement with the reported for PP/ZnO/GF [45] and $\mathrm{PP} / \mathrm{SiO}_{2} / \mathrm{GF}$ hybrids [46], where the combination of nano- and micro-fillers additionally increased the $T_{p}$ of the matrix, albeit the increments found in those hybrids $\left(\sim 7\right.$ and $6{ }^{\circ} \mathrm{C}$ at $2.0 \mathrm{wt} . \% \mathrm{ZnO}$ and 1.0 wt. $\% \mathrm{SiO}_{2}$ content, respectively) are smaller than the increases found for the same amount of IF-WS 2 . Further, $X_{c}$ of PP dropped upon incorporation of $\mathrm{ZnO}_{\mathrm{nO}} \mathrm{SiO}_{2}$ and GF, while the combined nucleating effect of IF-WS $/$ GF provoked a slight increase in crystallinity.

Table 1. Thermal stability, crystallization, and mechanical data for isotactic polypropylene (iPP) nanocomposites using nanoreinforcing fillers with different morphologies (e.g., tubular, spherical and laminar-like particles) taken from literature. $\Delta T_{10}=$ increment of degradation temperature for $10 \%$ weight loss, $\Delta T_{\mathrm{p}}=$ increment of crystallization peak temperature, $\delta E=$ Percentage variations of Young's modulus, $\delta \sigma_{\mathrm{y}}=$ Percentage variations of tensile strength and $\delta \varepsilon_{b}=$ Percentage variations of strain at yield.

\begin{tabular}{|c|c|c|c|c|c|c|}
\hline Filler & $\begin{array}{c}\text { Filler } \\
\text { content } \\
\text { (wt. \%) }\end{array}$ & $\begin{array}{l}\Delta T_{10} \\
\left({ }^{\circ} \mathrm{C}\right)\end{array}$ & $\begin{array}{l}\Delta T_{\mathrm{p}} \\
\left({ }^{\circ} \mathrm{C}\right)\end{array}$ & $\begin{array}{c}\delta E \\
(\mathbf{G P a})\end{array}$ & $\begin{array}{c}\delta \sigma_{\mathrm{y}} \\
(\mathrm{MPa}\end{array}$ & $\begin{array}{l}\delta \varepsilon \mathrm{b} \\
(\%)\end{array}$ \\
\hline \multirow[t]{3}{*}{ INT-MoS 2 [31] } & 0.1 & 54 & 3.9 & $15 \%$ & $13 \%$ & $-9 \%$ \\
\hline & 0.5 & 59 & 10 & $28 \%$ & $34 \%$ & $-18 \%$ \\
\hline & 1 & 59 & 10.1 & $40 \%$ & $41 \%$ & $-52 \%$ \\
\hline \multirow[t]{6}{*}{ HNTs [32-34] } & 1 & - & 3.9 & - & - & - \\
\hline & 2 & - & - & $32 \%$ & $22 \%$ & $-15 \%$ \\
\hline & 5 & - & 8.9 & - & - & - \\
\hline & 10 & 60 & 10 & - & - & - \\
\hline & 20 & - & 12.8 & - & - & - \\
\hline & 30 & 46 & 13.8 & - & - & - \\
\hline \multirow[t]{5}{*}{ CNTs [35-37] } & 0.1 & - & 7.6 & - & - & - \\
\hline & 0.25 & - & 8.4 & - & - & - \\
\hline & 0.5 & - & 10.7 & - & - & - \\
\hline & 1 & - & 10 & $23 \%$ & $15 \%$ & $-30 \%$ \\
\hline & 2 & 50 & - & - & - & - \\
\hline \multirow[t]{2}{*}{ rod- $\mathrm{Si}_{3} \mathrm{~N}_{4}[38]$} & 1 & - & 2 & $722 \%$ & $292 \%$ & - \\
\hline & 2 & - & 3 & - & - & - \\
\hline $\begin{array}{c}\text { Nanoclay (MMT) } \\
{[39-41]}\end{array}$ & 3 & 90 & 5 & $152 \%$ & $95 \%$ & $0 \%$ \\
\hline \multirow[t]{5}{*}{ IF-WS $2[16,42]$} & 0.1 & 11 & 9.8 & - & - & - \\
\hline & 1 & 14 & 13 & $39 \%$ & $41 \%$ & $-59 \%$ \\
\hline & 2 & 15 & 19 & - & - & - \\
\hline & 4 & 27 & 20.5 & - & - & - \\
\hline & 8 & 44 & 22.1 & - & - & - \\
\hline
\end{tabular}


Figure 3. DSC crystallization thermograms for neat iPP, PPS and some IF-WS reinforced multiscale laminates. The inset shows the crystallization peak temperature $T_{\mathrm{p}}$ vs. IF-WS 2 content.

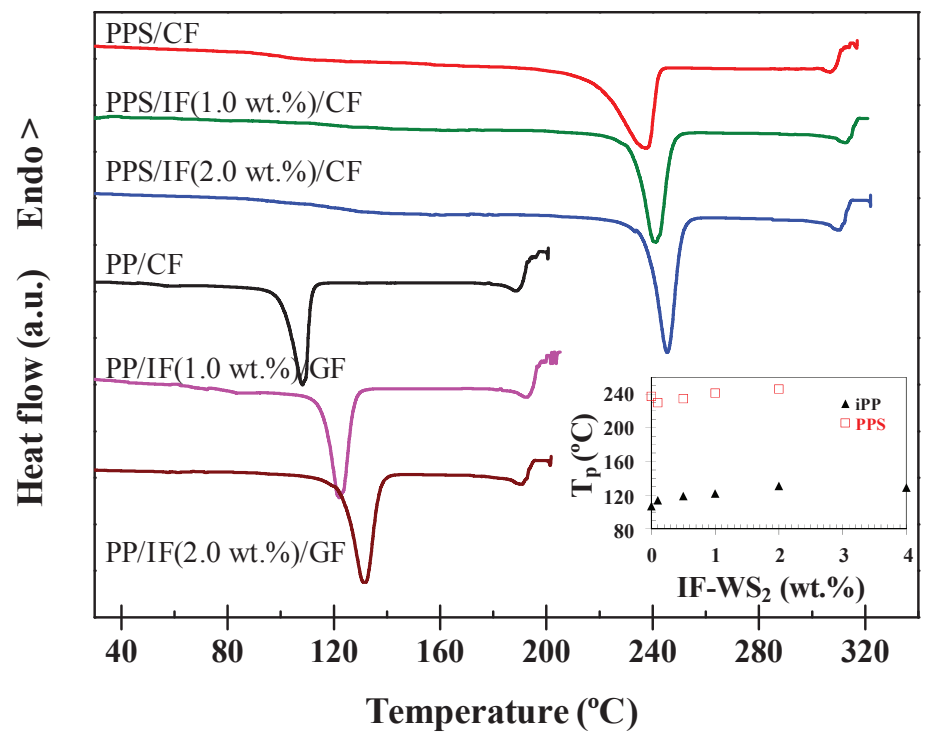

In this way, the control of the crystallization behavior has been shown to be a successful approach for improving physico-mechanical properties of polymer/INT nanocomposites. Table 1 summarizes the findings of several studies on the nucleating efficiency (NE) of nanoreinforcing fillers, and data can be compared by analyzing the difference between the crystallization peak temperature $\left(T_{p}\right)$ of each nanocomposite and that of the neat matrix ( $\left.\ddot{\mathrm{A}} \mathrm{T}_{\mathrm{p}}\right)$. Clearly, the $\ddot{\mathrm{A}} \mathrm{T}_{\mathrm{p}}$ value for INT-MoS $\mathrm{S}_{2}$ far exceeds the values observed for montmorillonite nanoclay [39] and rod-Si3 $\mathrm{N}_{4}$ [38], and is comparable to that observed for MWCNTs [35]. However, the nucleation efficiency of INT-MoS 2 is significantly lower in comparison to the value of $40 \%$ observed for inorganic fullerene-like $\mathrm{WS}_{2}$ nanoparticles at 1.0 wt.\% [16]. The results obtained clearly show that the addition of INT-WS 2 plays a remarkable role in accelerating the crystallization rate of iPP. In these systems, the crystallinity of iPP was found to rise up to $14 \%$ with increasing the INT-MoS content, from a value of $50 \%$ for iPP, to values of 54, 57 and 56\% for the nanocomposites with $0.1 \mathrm{wt} . \%, 0.5 \mathrm{wt} \%$ and $1 \mathrm{wt} \%$, respectively [25]. Furthermore, a new study on the crystallization behavior of biopolymer/INTs suggests that INT-WS 2 exhibits much more prominent nucleation activity on the crystallization of PHB than other specific nucleating agents or nano-sized fillers [29]. An increment of $35^{\circ} \mathrm{C}$ in the crystallization temperature of PHB was observed for as little as $0.1 \mathrm{wt} . \%$ INT-WS2. This corresponds to the highest value observed hitherto for PHB formulations using specific nucleating agents (e.g., talc, boron nitride lignin) or nano-sized fillers (e.g., CNTs, graphene oxide) [29]. 
Figure 4. Room temperature thermal conductivity of iPP and PPS-based laminates as a function of IF-WS 2 concentration.

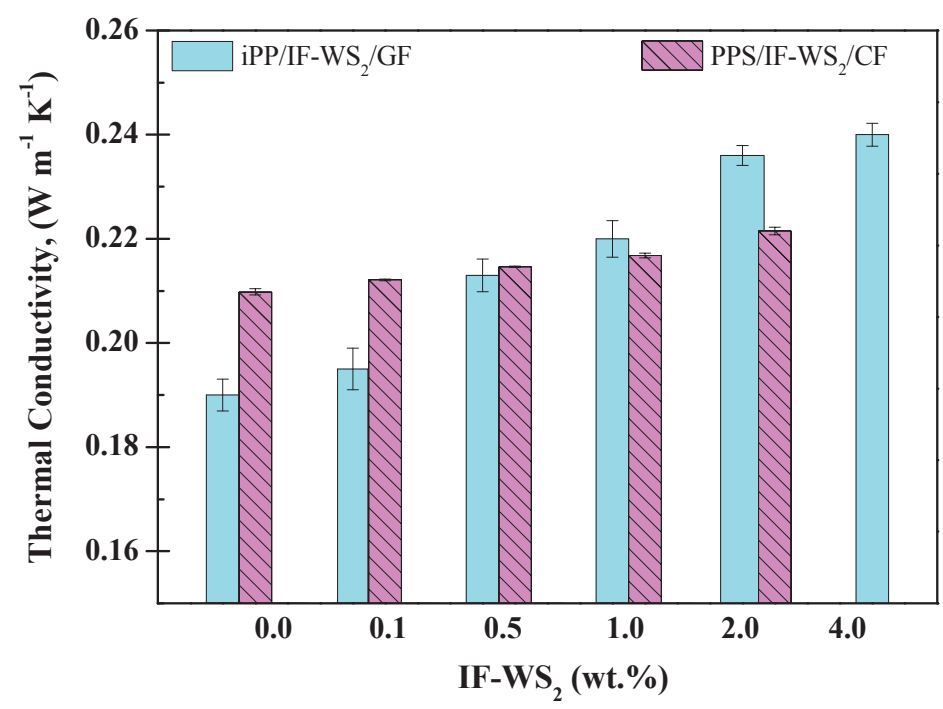

The addition of thermally conductive organic or inorganic nanofillers typically enhances the thermal conductivity $(\lambda)$ of polymers, which is interesting for applications that require effective dissipation of accumulated heat like connectors or thermal interface materials. It depends on several factors, namely the filler size, aspect ratio, concentration and state of dispersion, the nature, molecular weight and degree of crystallinity of the polymer, as well as the porosity of the material. The room temperature thermal conductivity of iPP- [22] and PPS- [24] based laminates has been measured in the transverse directions, and the results are shown in Figure 4. The incorporation of IF-WS2, which exhibit about twice the thermal conductivity of the neat matrices [47], results in significant $\lambda$ improvements in the case of iPP/GF laminates, up to $21 \%$ at $2.0 \mathrm{wt} . \%$ loading, whilst for PPS/CF composites the increments are smaller, about $9 \%$ for the same loading. This discrepancy is ascribed to the low thermal conductivity of the GF fabric $\left(\sim 0.05 \mathrm{~W} \mathrm{~m} \mathrm{~m}^{-1} \mathrm{~K}^{-1}\right)$ compared to that of $\mathrm{CF}\left(>200 \mathrm{~W} \mathrm{~m}^{-1} \mathrm{~K}^{-1}\right)$. It seems that the CFs play a dominating role in the thermal conductivity properties and mask the effect of the IF-WS 2 , as can be deduced from the comparison with the results of binary PPS/IF-WS 2 nanocomposites [48], where $\lambda$ rose by up to $\sim 45 \%$ upon addition of $2.0 \mathrm{wt} . \%$ IF-WS 2 . However, for iPP-based samples, the improvements in the hierarchical laminates are comparable to those reported for the corresponding binary composites [49], indicating that effect of the nanoparticles predominates. An analogous behavior has been reported for other hierarchical laminates based on thermoplastic polymers, such as PEEK/CNT/GF laminates [50], where $\lambda$ increased by $\sim 48 \%$ at $1.0 \mathrm{wt} . \% \mathrm{CNT}$, similarly to the enhancements found in the binary composites [51]. It is worthy to note that for the same nanofiller concentration, the increases in $\lambda$ upon addition of CNTs are only about double those achieved with the incorporation of the IF-WS 2 , while much higher differences would be expected considering the extraordinary high thermal conductivity of CNTs. The strong agglomerating tendency of CNTs, the small thermal conductance of the nanotube-polymer 
interface and the high interfacial thermal resistance between nanotubes within a bundle probably limits the property enhancement, whereas for composites incorporating IF-WS 2 the large nanofiller-matrix interfacial contact area and the very homogeneous dispersion lead to experimental $\lambda$ values even higher than the theoretical predictions.

\section{Mechanical Properties}

The dynamic mechanical properties of the multiscale composites were explored by DMA, technique that provides information about the viscoelastic behavior of the matrix, indicating changes in the stiffness and the relaxation processes that occur as a function of temperature. The influence of the IF-WS 2 on the dynamic mechanical behavior of polymer/IF-WS 2 nanocomposites has also been investigated [16-18]. In particular, it was observed that the improvements in the storage modulus values of PPS/IF-WS 2 nanocomposites are noticeably higher than those achieved in other thermoplastic nanocomposites based on IFs (e.g., iPP, nylon-6, PEEK), suggesting the presence of specific polymer-filler interactions in the case of PPS. The molecular nature of these interactions are still not understood, but they may be associated with the presence of outer S atoms on the IF nanoparticles, and more work is required in order to explain this phenomenon. Figure 5 presents the storage modulus $\left(E^{\prime}\right)$ and loss tangent $(\tan \delta)$ at the frequency of $1 \mathrm{~Hz}$ for PPS- and iPP-based composites incorporating 1.0 and $2.0 \mathrm{wt} . \% \mathrm{IF}-\mathrm{WS}_{2}$, and the glass transition temperature $\left(T_{\mathrm{g}}\right) v s$. nanoparticle content is shown in the inset of the Figure. Different behavior is also observed depending on the polymer matrix. Regarding PPS/CF laminates, the addition of very low IF-WS2 loadings (i.e., $0.1 \mathrm{wt} . \%)$ leads to a slight drop in $E^{\prime}\left(\sim 7 \%\right.$ at $\left.25^{\circ} \mathrm{C}\right)$, probably related to the decrease in the crystallinity found for this sample, as revealed by DSC analysis, since the crystalline regions enhance the modulus of semicrystalline polymers. The laminate incorporating $0.5 \mathrm{wt} . \% \mathrm{IF}-\mathrm{WS}_{2}$ exhibits similar $E^{\prime}$ to that of PPS/CF, since the reinforcement effect of the IF-WS 2 should compensate for the slight decrease in crystallinity. In contrast, the incorporation of nanoparticle contents $>0.5 \mathrm{wt} . \%$ leads to significant $E^{\prime}$ increments, by up to $22 \%$ for $2.0 \mathrm{wt} . \%$ nanoparticle content at $25{ }^{\circ} \mathrm{C}$. On the other hand, the gradual addition of IF-WS 2 to $\mathrm{iPP} / \mathrm{GF}$ results in progressive $E^{\prime}$ increases, by about $27 \%$ at $2.0 \mathrm{wt} . \%$ loading. This behavior is associated with the increase in crystallinity caused by heterogeneous nucleation, combined with an effective reinforcement effect arising from a very homogeneous nanoparticle dispersion. For both types of composites, the reinforcement effect is more pronounced at temperatures below $T_{g}$, in agreement with the behavior reported for PP/nanoclay/GF composites [30], where significant $E^{\prime}$ enhancements were found at low temperatures whereas the differences in modulus among the samples became insignificant at temperatures above the glass transition. 
Figure 5. Evolution of the (a) storage modulus $E^{\prime}$ and (b) tan $\delta$ as a function of temperature for the neat polymers and some multiscale laminates. The inset shows the glass transition temperature $T_{\mathrm{g}} v s$. IF-WS 2 content.
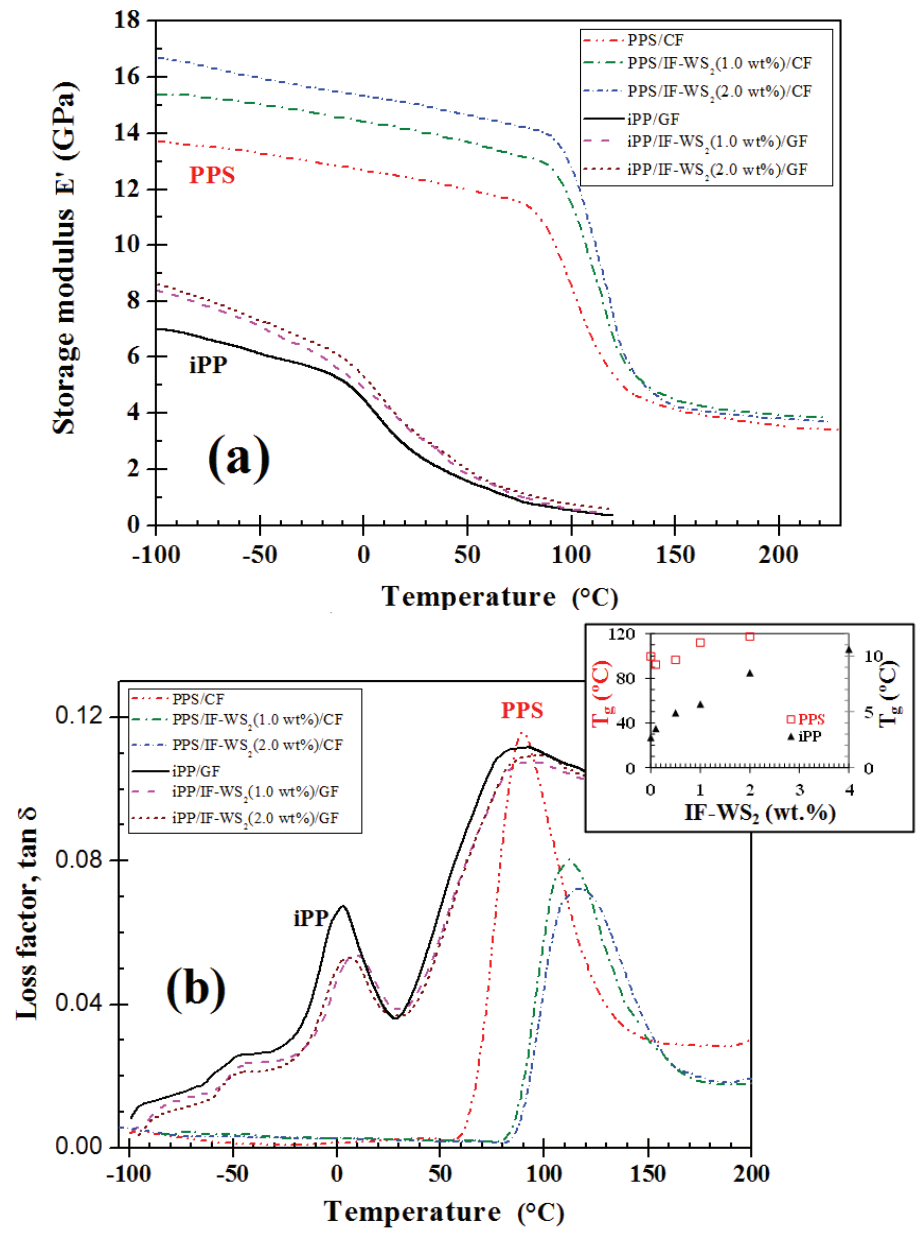

The evolution of $\tan \delta$ (ratio of the loss to storage modulus, a measure of the damping within the system) as a function of temperature (Figure 5b) exhibits an intense peak, named $\alpha$ relaxation that corresponds to the $T_{g}$. Further, the iPP/GF laminates show a peak at about $88{ }^{\circ} \mathrm{C}$ related to the relaxation of the crystalline phase $\left(\alpha_{c}\right)$. In an unfilled system, the polymer chain segments are free from restraints. The incorporation of fillers decreases the free volume and restricts the mobility of the matrix chains, which is reflected in higher $T_{g}$ values (see inset of Figure 5). Once again, different trend is found depending on the nature of the matrix. Thus, in the case of PPS/CF, the incorporation of low IF-WS 2 contents $\left(\leq 0.5\right.$ wt.\%) led to a downshift in $T_{g}$, while the addition of higher concentrations resulted in an upshift. As mentioned above, the addition of low nanoparticle loadings slows down the crystallization rate of PPS, leading to the formation of a more amorphous phase that provokes a slight drop in $T_{\mathrm{g}}$. However, the incorporation of higher contents has a nucleation effect, 
thereby raising the crystallinity of the polymer, which combined with a larger IF-WS - -matrix interfacial contact area results in an effective immobilization of the polymer chains, consequently an increase in $T_{g}$ of up to $18{ }^{\circ} \mathrm{C}$ at $2.0 \mathrm{wt} . \%$ IF-WS 2 . In contrast, the $T_{g}$ progressively increases upon addition of these nanoparticles to iPP/GF, the increment being about $6{ }^{\circ} \mathrm{C}$ for the same nanoparticle loading. In the same way, the presence of IF-WS $\mathrm{WS}_{2}$ causes an increase in the crystalline relaxation temperature $\alpha_{c}$ of iPP, since the strong nucleation effect of these nanoparticles accelerates the crystallization of iPP in the nanocomposites.

The magnitude of the $\tan \delta$ peak is indicative of the filler-matrix interactions. For both types of composites, the height of the tan $\delta$ peak decreases with increasing IF-WS 2 content, indicative of a strong nanofiller-matrix interfacial adhesion. Moreover, this reduction probably arises from a synergistic effect between the micro- and nano-fillers on restricting chain mobility, in agreement with the behavior reported for other GF-reinforced hierarchical composites [52]. The incorporation of both reinforcements has a strengthening effect, leading to a lower degree of molecular motion, hence, lower damping characteristics. It also noteworthy that the width of the tan $\delta$ peak becomes broader with increasing nanoparticle loading, phenomenon that can be interpreted as improved nanofiller-matrix interactions, and is another indication of the larger nanoparticle-matrix interfacial area. The IF-WS 2 and microscale fibers disturb the relaxation of the neighbour polymer chains, which would behave differently from those situated in the bulk matrix, resulting in a wider maximum. This behavior was also observed in IF-WS 2 reinforced iPP [16] and PEEK [18] nanocomposites, attributed to a more inhomogeneous amorphous phase in the composites in relation to the pure matrix.

The static mechanical properties of iPP and PPS based hybrid laminates have been investigated by tensile tests $[22,23]$, and the Young's modulus $(E)$, tensile strength $\left(\sigma_{\mathrm{y}}\right)$, elongation at break $\left(\varepsilon_{\mathrm{b}}\right)$, and toughness $(T)$ as a function of nanofiller loading are plotted in Figure 6 . The trends observed are similar to those described previously for the storage modulus. $E$ and $\sigma_{y}$ rise progressive with increasing nanoparticle loading in the case of iPP/GF composites, while they decrease slightly at low loadings and then grow in PPS/CF laminates, behavior that is directly related to the crystallinity of the samples, as discussed previously. Interestingly, both parameters only rise marginally upon addition of the IF-WS2, the maximum increments being $\sim 14 \%$ and $11 \%$ at 2.0 wt.\% nanoparticle content, respectively, in the case of PPS/CF, and even smaller for iPP/GF composites (Figure 6). However, considerably larger increases were observed for the binary iPP/IF- $\mathrm{WS}_{2}$ nanocomposites [49], where $E$ and $\sigma_{y}$ improved by around 42 and $31 \%$, respectively, for the indicated loading. For multiscale composites, it is expected that the nanofillers predominantly influence the properties that are matrix-dominated; consequently, only small increases are observed in the Young's modulus and tensile strength of the hybrids, since the tensile properties are more fiber-dominated. These results are consistent with the behavior reported for other thermoplastic-based hybrids [53], where $E$ and $\sigma_{y}$ of the fiber reinforced polymer only improved marginally upon incorporation of the nanoscale fillers due to the dominating role of the fibers.

With regard to the strain at break $(\varepsilon b)$, the trend found is very similar for both composite series. A moderate increase is found at low nanoparticle loadings, followed by a sharp reduction at higher concentrations. This indicates that higher amounts of IF-WS 2 hinder the ductile flow of the matrix. This tendency is in contrast to that typically reported for CNT-reinforced multiscale laminates [53], 
where $\varepsilon b$ systematically decreases upon addition of the carbon nanofillers, attributed to the presence of aggregates that produces stress concentrations at the filler-matrix interface, leading to premature failure. Similarly, Rahman et al. [30] found around 50\% reduction in tensile strain upon incorporation of $6.0 \mathrm{wt} . \%$ nanoclay to PP/GF (30 wt.\%), also ascribed to the poor nanoclay dispersion that strongly limits the plastic deformation of the matrix. The surprising behavior observed for the composites filled with IF-WS 2 is probably related to the lubricant character and more uniform dispersion of these inorganic nanoparticles combined with their spherical shape that reduce the stress concentration sites, thereby improving the matrix ductility. However, for IF-WS 2 concentrations higher than $1.0 \mathrm{wt} . \%$, a stiff hybrid network of micro- and nano-fillers could be formed that acts very effectively as a barrier for the mobility of the polymer chains, thus limiting the ductile deformation. A qualitatively similar behavior is found for the toughness, measured as the area under the tensile curve, that increases considerably at low IF-WS 2 loadings (i.e., by $35 \%$ at $0.1 \mathrm{wt} . \%$ content compared to $\mathrm{iPP} / \mathrm{GF}$ ) while drops moderately at concentrations higher than $1.0 \mathrm{wt} . \%$ (around 20\% decrease at $2.0 \mathrm{wt} . \%$ loading compared to PPS/CF). The small aggregates contribute to increase the brittleness under high strain rates, since they nucleate secondary cracks and favour the formation of dimples.

Figure 6. (a) Young's modulus $(E)$, (b) tensile strength $\left(\sigma_{y}\right)$, (c) elongation at break $(\varepsilon b)$ and (d) toughness $(T)$ as a function of IF-WS2 loading. Solid and open symbols correspond to PPS/IF-WS $/$ CF and $\mathrm{PPP} / \mathrm{IF}-\mathrm{WS}_{2} / \mathrm{GF}$ systems, respectively.
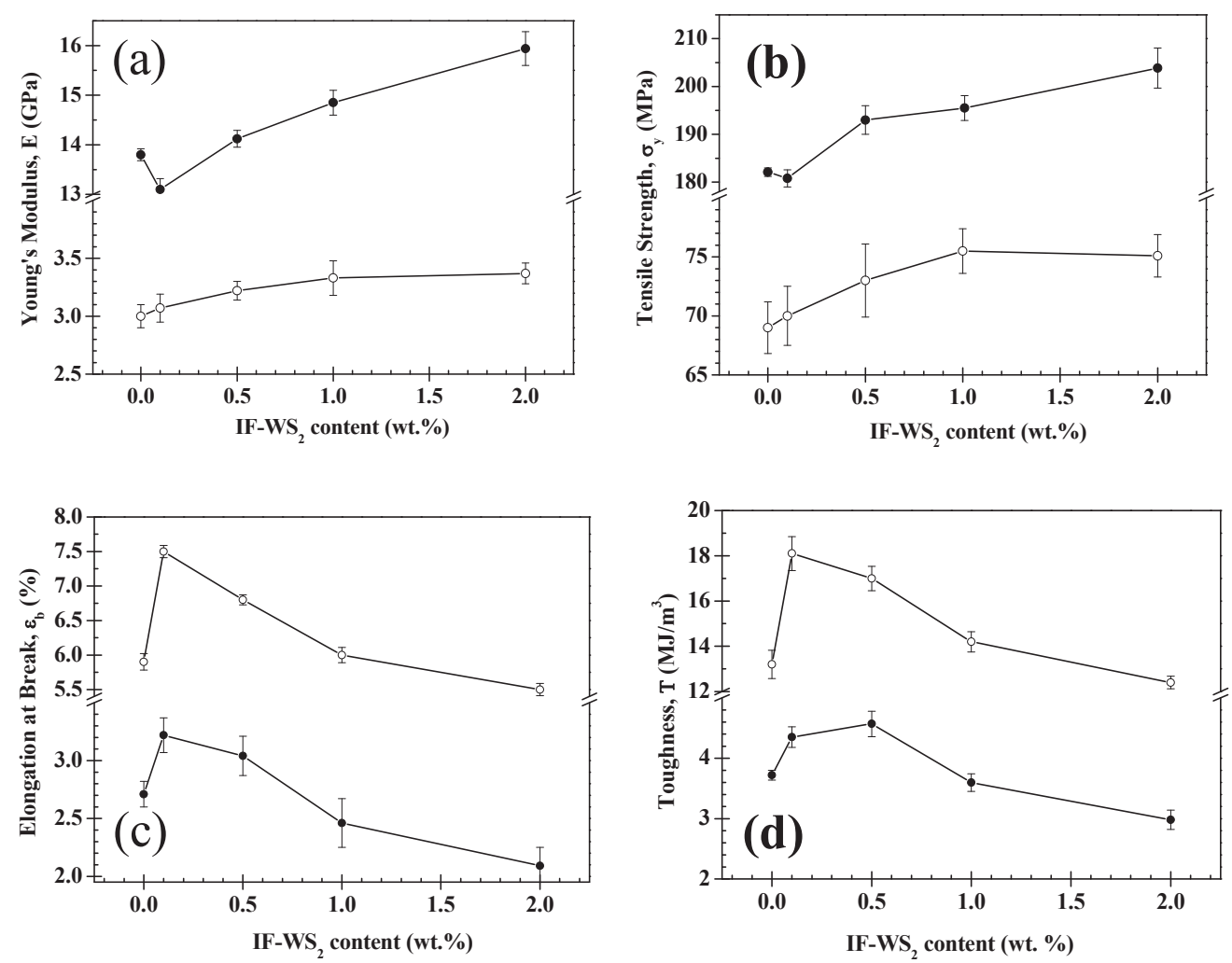
The influence of the IF-WS 2 on the flexural properties of iPP/GF and PPS/CF has also been investigated [22,24]. In this case, maximum increments in the flexural modulus Ef and flexural strength $\sigma_{\mathrm{fM}}$ of $\mathrm{iPP} / \mathrm{GF}$ up to 26 and $22 \%$, respectively, have been attained at $2.0 \mathrm{wt} . \%$ loading. Similarly, enhancements of 25 and 15\% have been found in PPS/CF composites for the same nanofiller loading. The comparison of the results with those obtained for the corresponding binary nanocomposites $[22,24]$ reveals a synergistic effect of both fillers on enhancing the flexural properties of the matrix.

Table 2. Comparison of the increment in static mechanical properties (in \%) for different polypropylene (PP) and polyphenylene sulfide-(PPS) based hierarchical laminates. MWCNT: multi-walled carbon nanotubes; MMT: montmorillonite; Woll: Wollastonite; $E$ : Young's modulus; $\sigma_{\mathrm{y}}$ : tensile strength at yield; $G$ : impact strength; $E_{\mathrm{f}}$ : flexural modulus; $\sigma \mathrm{fm}$ : flexural strength.

\begin{tabular}{|c|c|c|c|c|c|c|c|c|}
\hline Matrix & $\begin{array}{c}\text { Fiber } \\
\text { (wt.\%) }\end{array}$ & $\begin{array}{c}\text { CNT } \\
\text { (wt.\%) }\end{array}$ & $\Delta E(\%)$ & $\begin{array}{c}\Delta \sigma_{\mathrm{y}} \\
(\mathrm{MPa})\end{array}$ & $\begin{array}{c}\Delta T \\
(\%)\end{array}$ & $\begin{array}{l}\Delta \boldsymbol{E}_{\mathrm{f}} \\
(\%)\end{array}$ & $\begin{array}{l}\Delta \sigma_{\mathrm{fM}} \\
(\%)\end{array}$ & Ref. \\
\hline PP & $\begin{array}{l}\text { GF } \\
(5)\end{array}$ & MWCNTs & 40 & 39 & 24 & 36 & 43 & {$[18]$} \\
\hline PP & $\begin{array}{l}\mathrm{CF} \\
(5)\end{array}$ & MWCNTs & 57 & 37 & 34 & 51 & 35 & [18] \\
\hline PP & $\begin{array}{l}\text { GF } \\
(30)\end{array}$ & $\begin{array}{l}\text { MMT } \\
(6)\end{array}$ & 6 & 6 & - & 9 & 10 & [3] \\
\hline PP & $\begin{array}{l}\text { GF } \\
(30)\end{array}$ & $\begin{array}{l}\text { Woll } \\
(10)\end{array}$ & -6 & -6 & -31 & -2 & -3 & [19] \\
\hline PP & $\begin{array}{c}\text { GF } \\
(40)\end{array}$ & $\begin{array}{c}\mathrm{SiO}_{2} \\
\text { (1) }\end{array}$ & 22 & 3 & -5 & 2 & 12 & [9] \\
\hline PP & $\begin{array}{l}\text { GF } \\
(30)\end{array}$ & $\begin{array}{c}\text { IF-WS } \\
\text { (2) }\end{array}$ & 10 & 8 & - & 26 & 22 & [1] \\
\hline PPS & $\begin{array}{l}\text { GF } \\
(40)\end{array}$ & $\begin{array}{c}\mathrm{CaCO}_{3} \\
(3 \text { wt. } \%)\end{array}$ & 27 & 9 & 14 & - & - & [20] \\
\hline PPS & $\begin{array}{c}\text { GF } \\
(40)\end{array}$ & $\begin{array}{c}\mathrm{CaCO}_{3} \\
\text { (3) }\end{array}$ & - & - & 20 & 0 & 3 & [21] \\
\hline PPS & $\mathrm{CF}$ & $\begin{array}{c}\text { IF-WS } \\
\text { (2) }\end{array}$ & 14 & 11 & -20 & 25 & 15 & {$[2,4]$} \\
\hline
\end{tabular}

Table 2 compares the improvements in static mechanical properties reported for various PP and PPS-based hierarchical composites [30,46,54-57]. Clearly, the highest improvements are attained upon addition of multi-walled carbon nanotubes (MWCNTs) to fiber-reinforced PP composites [54], which is reasonable taking into account the very high modulus of these carbon nanofillers. Nevertheless, among the various inorganic fillers, IF-WS 2 lead to larger stiffness and strength improvements than montmorillonite [30], wollastonite [55], or nanosilica [46], and comparable to those of $\mathrm{CaCO}_{3}[56,57]$.

In the same way, the incorporation of INTs can also lead to improvement in the mechanical properties of polymer/INTs $[25,27]$. As an example, the characteristic mechanical data (e.g., Young's modulus, $E$, tensile strength, $\sigma_{\mathrm{y}}$ and strain at yield, $\varepsilon_{\mathrm{y}}$ ) for the PP nanocomposites incorporating 
nanoreinforcing fillers with different morphologies are summarized in Table 1 [31-42]. It can be observed that the addition of INT-MoS 2 progressively enhances the Young's modulus of the matrix, with increments of 15,28 , and $40 \%$ for loading fractions of $0.1,0.5$, and $1.0 \mathrm{wt} . \%$, respectively. The improved $E$ obtained in this work is ascribed to the very uniform dispersion of the INT-MoS 2 and their high aspect ratio, which results in larger nanofiller-polymer interfacial area. Qualitatively similar trends were found for the tensile strength, where the increments were around 13, 34, and $41 \%$ for the abovementioned nanofiller contents. On the other hand, the incorporation of the inorganic nanotubes leads to a slight decrease in $\varepsilon_{\mathrm{y}}$. This is a typical behavior of nanofiller-reinforced polymer composites, since the nanofillers restrict the ductile flow of the matrix, and is in agreement with the results reported by Lopez-Gaxiola et al. [58] for carbon filler-reinforced PP composites. Table 1 also shows the percentage variations in the mechanical properties of iPP nanocomposites containing similar amounts $(\sim 1.0 \mathrm{wt} . \%)$ of various nanofillers. Remarkable improvements in the mechanical properties are observed for iPP/INT-MoS2, where the non-modified nanofillers were dispersed uniformly in the iPP matrix for all the compositions prepared [31]. The magnitude of increase in the modulus and strength is similar to that obtained for IF-WS 2 nanoparticles [42] and far exceeds that reported for both modified HNTs [34] and CNTs [37]. However, silicon nitrides clearly provide the best reinforcement for PP matrix, which has been related to the alignment and exfoliation of rod-shaped $\mathrm{Si}_{3} \mathrm{~N}_{4}$ particles [38]. These phenomena were also mainly responsible for the $95 \%$ enhancement in the tensile strength and $152 \%$ increase in the tensile modulus of PP using p-aminobenzoic acid modified-clay with PP-g-MA as a compatibilizer [40]. On the other hand, Reddy et al. have reported that the high rigidity of INT-WS 2 and the effective load transfer from the matrix to the INT-WS 2 were responsible for the improved mechanical properties of PMMA/INT-WS 2 nanocomposites [27]. In particular, it was observed that the elastic modulus of PMMA fiber meshes was increased by 10 and 22 times upon incorporation along the fiber axis of 1.0 and $2.0 \mathrm{wt} . \%$ INT-WS2, respectively. Analogously, the tensile strength of the composite fibers increased by 35 and $32 \%$ for the indicated nanoparticle loadings. However, the toughness of the sample with $2.0 \mathrm{wt} . \%$ INT-WS $\mathrm{W}_{2}$ was lower than that of the neat PMMA fiber, since nanofiller aggregation started to take place. Overall, experimental results point out the advantages of using these environmentally friendly and cheap inorganic fullerenes and nanotubes instead of conventional nanoparticles for improving the mechanical performance of thermoplastic composites.

\section{Tribological Properties}

Inorganic nanoparticles are frequently incorporated into thermoplastic polymers with the aim to improve the tribological properties. The nanoparticles exhibit some advantages compared to conventional microfillers, such as higher specific surface area, lower abrasiveness due to a reduced angularity, enhanced strength, modulus and toughness. In addition, IF-WS 2 possess a lubricant character, and have been shown to be effective for improving the tribological properties of thermoplastic polymers such as PPS or PEEK $[59,60]$. Figure 7 displays the change in the coefficient of friction $(\mu)$ and wear rate of PPS/CF upon addition of IF-WS 2 [23]. The incorporation of $0.1 \mathrm{wt} . \%$ IF-WS 2 leads to a slight increase in $\mu(\sim 5 \%)$ compared to the reference laminate, probably related to the decrease in stiffness and strength found for this sample that prevails over the lubricant effect of the IF-WS 2 . Further 
increasing the nanoparticle loading, $\mu$ drops strongly, reaching the lowest value at $2.0 \mathrm{wt} . \% \mathrm{IF}-\mathrm{WS}_{2}$ (about 32\% drop compared to the reference laminate). Rapoport et al. [61] proposed a rolling mechanism for these nanoparticles, in which they act as a ball-bearing component, implying that they roll instead of sliding between the surfaces, hence, decreasing the shear stress, contact temperature and coefficient of friction. Likewise, the abovementioned behavior can be attributed to a synergistic effect between the CFs and the inorganic nanoparticles, as reported previously for CF-reinforced PEEK incorporating $\mathrm{ZnS}$ or $\mathrm{TiO}_{2}$ nanoparticles [62].

Figure 7. Coefficient of friction and wear rate of PPS/IF-WS $2 / \mathrm{CF}$ laminates as a function of IF-WS2 content.

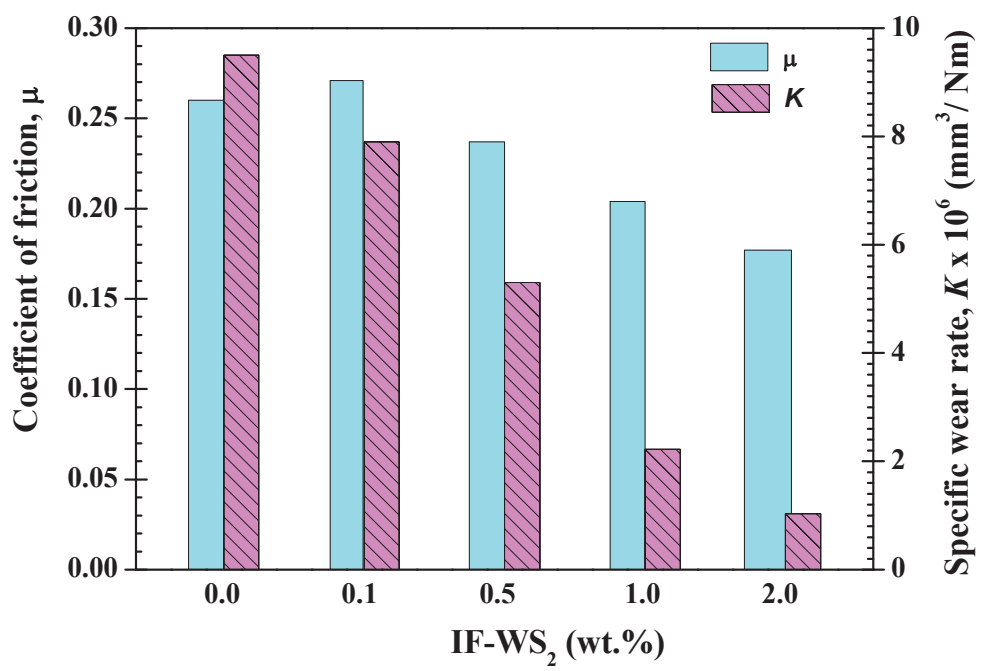

With regard to the wear rate, a progressive reduction in this parameter is found upon increasing IF-WS 2 concentration, which decreases by nine-fold for the composite with $2.0 \mathrm{wt} \%$ loading compared to the reference laminate. This increase in wear resistance has been attributed to the formation of a thin, continuous, and smooth transfer film on the counterface during sliding combined with the reinforcing effect, and it is enhanced by the presence of the two fillers. The adhesion of the transfer film would be stronger since a homogeneous mixture of the debris is formed, and the resistance to cracking and fatigue failure would also increase in the presence of the nanoparticles. An analogous trend was reported for the wear behavior of $\mathrm{PEEK} / \mathrm{ZrO}_{2} / \mathrm{CF}$ composites [62], where a synergistic effect of $\mathrm{CFs}$ with $\mathrm{ZrO}_{2}$ nanoparticles on enhancing the matrix wear resistance was proposed. Overall, the combination of conventional CF-reinforced thermoplastics with lubricant nanoparticles like IF-WS 2 is a promising approach to develop multiscale hybrids with superior tribological performance. 
Table 3. Wear rate (K) data of PP nanocomposites nanocomposites using nanoreinforcing fillers with different morphologies.

\begin{tabular}{|c|c|c|c|}
\hline Filler & $\begin{array}{c}\text { Filler } \\
\text { content } \\
\text { (wt.\%) }\end{array}$ & $\begin{array}{c}\text { Wear rate } \\
(K) \times 10^{4} \\
\left(\mathrm{~mm}^{3} / \mathrm{Nm}\right)\end{array}$ & $\begin{array}{c}\text { Percentage variation } \\
\text { of } K(\%)\end{array}$ \\
\hline \multirow[t]{4}{*}{ INT-MoS 2 [31] } & 0 & 6.27 & - \\
\hline & 0.1 & 5.97 & 5 \\
\hline & 0.5 & 4.35 & 31 \\
\hline & 1 & 2.98 & 53 \\
\hline Nanoclay [65] & 1 & - & 38.5 \\
\hline IF-WS ${ }_{2}[42]$ & 1 & - & 63 \\
\hline
\end{tabular}

Table 3 collects the wear rate of melt-procesable iPP/INT-MoS2 nanocomposites [31]. With the incorporation of INT- $\mathrm{MoS}_{2}$ the wear resistance of the polymer is considerably enhanced and the nanocomposite with $1.0 \mathrm{wt} \%$ loading shows a reduction of about $53 \%$. These inorganic nanotubes dispersed in the polymer matrix can act as a barrier and prevent large-scale fragmentation of the iPP. It has been reported that nanofillers of similar dimensions as the segments of the surrounding polymer chains enable a milder material removal and aid the formation of uniform tenacious transfer film $[63,64]$. Table 3 also compares the percentage of variations in the wear rate of PP nanocomposites containing 1.0 wt. $\%$ of nanoclay [65], IF-WS 2 [42], and INT-MoS [31]. In particular, PP/INT-MoS2 showed higher wear property improvement than that of PP/nanoclay without the need for an exfoliation process. The highest percentage of improvement in wear rate is found for IF-WS 2 solid lubricant nanoparticles, which have recently been identified as ideal candidates for improving the tribological performance of polymers like epoxy [61], nylon-6 [19], and PEEK [18].

\section{Conclusions and Future Developments}

The addition of IF/INTs has been demonstrated to be a very efficient strategy to improve the thermal, mechanical and tribological properties of thermoplastic polymers like iPP, PPS, or PEEK and their fiber-reinforced composites. These materials can be fabricated by simple melt-processing and compression molding without the need for modifiers or surfactants, leading to a very homogenous dispersion of the nanofillers within the matrix. More importantly, they exhibit similar or enhanced performance when compared with composites that incorporate CNTs, nanoclays or other inorganic spherical nanoparticles, but are substantially more cost-effective, efficient and environmentally-friendly. Results demonstrate the existence of synergistic effects of both micro-and nanoscale fillers on enhancing the stiffness, strength, thermal conductivity, thermal stability, flammability, and wear resistance of hierarchical thermoplastic-based composites. This new family of materials has a wide range of potential applications ranging from medicine to the aerospace, automotive, and electronics industries. Some of these applications are still at an early stage of research and development. However, for optimal control of the properties of these new materials, it is highly important to tailor the fabrication process from the viewpoint of the final product. In particular, the improvement and application of these nanocomposites in comparison with other organic-inorganic hybrid nanomaterials (silica, metal oxides, clays, etc.) depend on how effectively we optimize and scale-up 
their fabrication method. For specific applications, these nanoparticles should be surface functionalized in order to confer more selectivity, specificity and reactivity with the polymer chains. An additional demanding area is the potential of these nanoparticles in the field of biocompatible and/or biodegradable polymeric composites for packaging and medical applications and their eventual toxicological effects, if any, need to be investigated. Research and progress in these areas will not only benefit the current applications but would also lead to new markets as well as to future development of diverse hierarchical thermoplastic-based composites.

\section{Acknowledgments}

This work was supported by the Spanish Ministry Economy and Competitivity (MINECO), Project MAT-2010-21070-C02-01. Dr. M. Naffakh would like to acknowledge the Ministerio de Economía y Competitividad (MINECO) for a "Ramón y Cajal" Senior Research Fellowship and Ana Diez-Pascual wishes to acknowledge the CSIC for a JAE Postdoctoral Fellowship cofinanced by the EU.

\section{Author Contributions}

This project was conceived and designed by MN. AD characterized and discussed the mechanical and tribological properties. MN analyzed and discussed the morphology and thermal properties. Both authors contributed in writing this paper.

\section{Conflicts of Interest}

The authors declare no conflict of interest.

\section{References}

1. Offringa, A.R. Thermoplastic composites in aerospace-proven through cost-effective processing. In Proceedings of the 41th International Conference on Automated Composites (ICAC), Nottingham, UK, 6-7 September 1995.

2. Henshaw, J.M.; Han, W.J.; Owens, A.D. An overview of recycling issues for composite materials. J. Thermoplast. Compos. Mater. 1996, 1, 4-20.

3. Marsh, G. Next step for automotive materials. Mater. Today 2003, 6, 36-43.

4. Sanchez, C.; Belleville, P.; Polpall, M.; Nicole, L. Applications of advanced hybrid organic-inorganic nanomaterials: From laboratory to market. Chem. Soc. Rev. 2011, 40, 696-753.

5. Qian, H.; Greenhalgh, E.S.; Shaffer, M.S.P.; Bismarck, A. Carbon nanotube-based hierarchical composites: A review. J. Mater. Chem. 2010, 20, 4751-4762.

6. Lin, G.; Xie, G.; Sui, G.; Yang, R. Hybrid effect of nanoparticles with carbon fibers on the mechanical and wear properties of polymer composites. Compos. Part B 2012, 43, 44-49.

7. Hussain, M.; Nakahira, A.; Nishijima, S.; Niihara, K. Evaluation of machanical behaviour of CFRC transverse to the fiber direction at room and cryogenic temperature. Compos. Part A 2000, 31, 173-179. 
8. Timmerman, J.F.; Hayes, B.S.; Seferis, J.C. Nanoclay reinforcement effects on the cryogenic microcraking of carbon fiber/epoxy composites. Compos. Sci. Technol. 2002, 62, 1249-1258.

9. Tenne, R. Inorganic nanotubes and fullerene-like nanoparticles. Nat. Nanotechnol. 2006, 1, $103-111$.

10. Tenne, R.; Redlich, M. Recent progress in the research of inorganic fullerene-like nanoparticles and inorganic nanotubes. Chem. Soc. Rev. 2010, 39, 1423-1434.

11. Tenne, R.; Margulis, L.; Genut, M.; Hodes, G. Polyhedral and cylindrical structures of WS2. Nature 1992, 360, 444-445.

12. Margulis, L.; Salitra, G.; Tenne, R.; Talianker, M. Nested fullerene-like structures. Nature 1993, 365,113-114.

13. Naffakh, M.; Díez-Pascual, A.M.; Marco, C.; Ellis, G.; Gómez-Fatou, M.A. Opportunities and challenges in the use of inorganic fullerene-like nanoparticles to produce advanced polymer nanocomposites. Prog. Polym. Sci. 2013, 38, 1163-1231.

14. Kickelbick, G. Concepts for the incorporation of inorganic building blocks into organic polymers on a nanoscale. Prog. Polym. Sci. 2003, 28, 83-114.

15. Rozenberg, B.A.; Tenne, R. Polymer-assisted fabrication of nanoparticles and nanocomposites. Prog. Polym. Sci. 2008, 33, 40-112.

16. Naffakh, M.; Martin, Z.; Fanegas, N.; Marco, C.; Gómez, M.A.; Jiménez, I. Influence of inorganic fullerene-like $\mathrm{WS}_{2}$ nanoparticles on the thermal behavior of isotactic polypropylene. J. Polym. Sci. B 2007, 45, 2309-2321.

17. Naffakh, M.; Marco, C.; Gómez, M.A.; Gómez-Herrero, J.; Jiménez, I. Use of inorganic fullerene-like $\mathrm{WS}_{2}$ to produce new high-performance polyphenylene sulfide nanocomposites: Role of the nanoparticle concentration. J. Phys. Chem. B 2009, 113, 10104-10111.

18. Naffakh, M.; Díez-Pascual, A.M.; Marco, C.; Gómez, M.A.; Jiménez, I. Novel melt-processable poly(ether ether ketone) (PEEK)/inorganic fullerene-like $\mathrm{WS}_{2}$ nanoparticles for critical applications. J. Phys. Chem. B 2010, 114, 11444-11453.

19. Naffakh, M.; Marco, C.; Gomez, M.A.; Jimenez, I. Novel melt-processable nylon-6/inorganic fullerene-like $\mathrm{WS}_{2}$ nanocomposites for critical applications. Mater. Chem. Phys. 2011, 129, 641-648.

20. Naffakh, M.; Marco, C.; Ellis, G. Novel polypropylene/inorganic fullerene-like $\mathrm{WS}_{2}$ nanocomposites containing a $\beta$-nucleating agent: Dynamic crystallization and melting behaviour. J. Phys. Chem. B 2011, 115, 10836-10843.

21. Naffakh, M.; Díez-Pascual, A.M.; Gómez-Fatou, M.A. New hybrid nanocomposites containing carbon nanotubes, inorganic fullerene-like $\mathrm{WS}_{2}$ nanoparticles and poly(ether ether ketone) (PEEK). J. Mater. Chem. 2011, 21, 7425-7433.

22. Díez-Pascual, A.M.; Naffakh, M. Tuning the properties of carbon fiber-reinforced poly(phenylene sulphide) laminates via incorporation of inorganic nanoparticles. Polymer 2012, 53, 2369-2378.

23. Díez-Pascual, A.M.; Naffakh, M. Polypropylene/glass fiber hierarchical composites incorporating inorganic fullerene-like nanoparticles for advanced technological applications. ACS Appl. Mater. Interfaces 2013, 5, 9691-9700. 
24. Díez-Pascual, A.M.; Naffakh, M. Inorganic nanoparticle-modified carbon fiber fabric/poly(phenylene sulphide) laminates with enhanced thermomechanical behaviour. Materials 2013, 6, 3171-3193.

25. Naffakh, M.; Remškar, M.; Marco, C.; Gómez, M.A.; Jiménez, I. Towards a new generation of polymer nanocomposites based on inorganic nanotubes. J. Mater. Chem. 2011, 21, 3574-3578.

26. Zohar, E.; Baruch, S.; Shneider, M.; Dodiu, H.; Kenig, S.; Wagner, D.H.; Zak, A.; Moshkovith, A.; Rapoport, L.; Tenne, R. The Mechanical and Tribological Properties of Epoxy Nanocomposites with $\mathrm{WS}_{2}$ Nanotubes. Sens. Transducers J. 2011, 12, 53-65.

27. Reddy, C.S.; Zak, A.; Zussman, E. $\mathrm{WS}_{2}$ nanotubes embedded in PMMA nanofibers as energy absorptive material. J. Mater. Chem. 2011, 21, 16086-16093.

28. Lalwani, G.; Henslee, A.M.; Farshid, B.; Parmar, P.; Lin, L.; Qin, Y.X.; Kasper, F.K.; Mikos, A.G.; Sitharaman, B. Tungsten disulfide nanotubes reinforced biodegradable polymers for bone tissue engineering. Acta Biomater. 2013, 9, 8365-8373.

29. Naffakh, M.; Marco, C.; Ellis, G. Inorganic WS 2 Nanotubes that improve the crystallization behavior of biodegradable poly(3-hydroxybutyrate). Cryst. Eng. Comm. 2013, 16, 1126-1135.

30. Rahman, N.A.; Hassan, A.; Yahya, R.; Lafia-Araga, R.A.; Hornsby, P.R. Polypropylene/glass fiber/nanoclay hybrid composites: morphological, thermal, dynamic mechanical and impact behaviors. J. Reinf. Plast. Compos. 2012, 31, 269-281.

31. Naffakh, M.; Díez-Pascual, A.M.; Remškar, M.; Marco, C. New inorganic nanotube polymer nanocomposites: Improved thermal, mechanical and tribological properties in isotactic polypropylene incorporating INT-MoS2. J. Mater. Chem. 2012, 22, 17002-17010.

32. Liu, M.; Guo, B.; Du, M.; Chen, F.; Jia, D. Halloysite nanotubes as a novel $\beta$ - nucleating agent for isotactic polypropylene. Polymer 2009, 50, 3022-3030.

33. Du, M.; Guo, B.; Jia, D. Thermal stability and flame retardant effects of halloysite nanotubes on polypropylene. Eur. Polym. J. 2006, 42, 1362-1369.

34. Prashantha, K.; Lacrampe, M.F.; Krawczak, P. Processing and characterization of alloysite nanotubes filled polypropylene nanocomposites based on a masterbatch route: Effect of halloysites treatment on structural and mechanical properties. Express Polym. Lett. 2011, 5, 295-307.

35. Marco, C.; Naffakh, M.; Gómez, M.A.; Santoro, G.; Ellis, G. The crystallization of polypropylene in multiwall carbon nanotube based composites. Polym. Compos. 2011, 32, 324-333.

36. Jin, S.H.; Kang, C.H.; Yoon, K.H.; Bang, D.S.; Park, Y.B. Effect of compatibilizer on morphology, thermal and rheological properties of polypropylene/functionalized multi-walled carbon nanotubes composite. Appl. Polym. Sci. 2009, 111, 1028-1033.

37. Lui, Y.; Gao, J. Mechanical properties and wear behavior of polypropylene/carbon nanotube nanocomposites. Adv. Mater. Res. 2011, 798, 299-300.

38. Rangari, V.K.; Shaik, Y.M.; Mohammad, G.M.; Jeelani, S. Reinforcement of $\mathrm{Si}_{3} \mathrm{~N}_{4}$ nanoparticles in Polypropylene single fibers through melt extrusion process and their properties. J. Appl. Polym. Sci. 2011, 121, 1512-1520. 
39. Xu, W.; Ge, M.; He, P. Nonisothermal crystallization kinetics of polypropylene/montmorillonite nanocomposites. J. Polym. Sci. Polym. Phys. 2002, 40, 408-414.

40. Sharma, S.K.; Nayak, S.K. Surface modified clay/polypropylene (PP) nanocomposites: Effect on physico-mechanical, thermal and morphological properties. Polym. Degrad. Stab. 2009, 94, $132-138$.

41. Sharma, S.K.; Nema, A.K.; Nayak, S.K. Polypropylene nanocomposite film: A critical evaluation on the effect of nanoclay on the mechanical, thermal, and morphological behavior. J. Appl. Polym. Sci. 2010, 115, 3463-3473.

42. Naffakh, M.; Díez-Pascual, A.M.; Marco, C.; Ellis, G. Novel polypropylene/inorganic fullerene-like $\mathrm{WS}_{2}$ nanocomposites containing a $\beta$-nucleating agent: Mechanical, tribological and rheological properties. Mater. Chem. Phys. 2014, 144, 98-106.

43. Gao, F.G.; Beyer, G.; Yuan, Q.C. Mechanistic study of fire retardancy of carbon nanotube/ethylene vinyl acetate copolymers and their clay composites. Polym. Deg. Stab. 2005, 89, 559-564.

44. Haiyun, M.; Lifang, T.; Zhongbin, X.; Zhengping, F. Synergistic effect of carbon nanotube and clay for improving the flame retardancy of ABS resin. Nanotechnology 2007, 18, 375602-375610.

45. Cui, Y.H.; Wang, X.X.; Li, Z.Q.; Tao, J. Fabrication and properties of Nano-ZnO/Glass fiber reinforced polypropylene composites. J. Vinyl. Addit. Technol. 2010, 16, 189-194.

46. Jacob, S.; Suma, K.K.; Mendaz, J.M.; George, A.; George, K.E. Modification of polypropylene/glass fiber composites with nanosilica. Macromol. Symp. 2009, 277, 138-143.

47. Díez-Pascual, A.M.; Naffakh, M.; Gómez-Fatou, M.A. Mechanical and electrical properties of novel poly(ether ether ketone)/carbon nanotube/inorganic fullerene-like $\mathrm{WS}_{2}$ hybrid nanocomposites: experimental measurements and theoretical predictions. Mater. Chem. Phys. 2011, 130, 126-133.

48. Naffakh, M.; Díez-Pascual, A.M.; Marco, C.; Ellis, G. Morphology and thermal properties of novel poly(phenylene sulfide) hybrid nanocomposites based on single-walled carbon nanotubes and inorganic fullerene-like WS2 nanoparticles. J. Mater. Chem. 2012, 22, 1418-1425.

49. Díez-Pascual, A.M.; Naffakh, M. Mechanical and thermal behaviour of isotatic polypropylene reinforced with inorganic fullerene-like IF-WS 2 nanoparticles: Effect of filler loading and temperature. Mater. Chem. Phys. 2013, 141, 979-989.

50. Ashrafi, B.; Diez-Pasual, A.M.; Johnson, L.; Genest, M.; Hind, S.; Martínez-Rubi, M.; González-Domínguez, J.M.; Martínez, M.T.; Simard, B.; Gómez, M.A.; et al. Influence of interfacial characteristics on properties of PEEK/Glass fiber laminates modified with single-walled carbon nanotubes. Compos. Part A 2012, 43, 1267-1279.

51. Díez-Pascual, A.M.; Naffakh, M.; Gómez, M.A.; Marco, C.; Ellis, G.; Martinez, M.T.; Anson, A.; Gónzaelz-Dominguez, J.M.; Martinez-Rubi, Y.; Simard, B. The influence of a compatibilizer on the thermal and dynamic mechanical properties of PEEK/carbon nanotube composites. Nanotechnology 2009, 20, doi:10.1088/0957-4484/20/31/315707. 
52. Díez-Pascual, A.M.; Ashrafi, B.; Naffakh, M.; González-Domínguez, J.M.; Johnston, A.; Simard, B.; Martínez, M.T.; Gómez, M.A. Influence of carbon nanotubes on the thermal, electrical and mechanical properties of PEEK/glass fiber laminates. Carbon 2011, 49, 2817-2833.

53. Díez-Pascual, A.M.; Naffakh, M.; Marco, C.; Gómez, M.A.; Ellis, G. Multiscale fiber-reinforced thermoplastic composites incorporating carbon nanotubes: A review. Curr. Opin. Mater. Sci., in press.

54. Rahmanian, S.; Thean, K.S.; Suraya, A.R.; Shazed, M.A.; Salleh, M.A.M.; Yusoff, H.M. Carbon and glass hierarchical fibers: Influence of carbon nanotubes on tensile, flexural and impact properties of short fiber reinforced composites. Mater. Des. 2013, 43, 10-16.

55. Joshi, H.; Purnima, J. Development of glass fiber, wollastonite reinforced polypropylene hybrid composite: Mechanical properties and morphology. Mater. Sci. Eng. A 2010, 527,1946-1951

56. Haribabu, J.; Sir, K.A.; Ravikiran, B. Fabrication and characterization of PPS $/ 40 \%$ GF $/$ nano-CaCO $\mathrm{CaC}_{3}$ hybrid composites. Int. J. Mod. Eng. Res. 2013, 3, 2262-2266.

57. Narayana, S.; Suman, K.N.S.; Babu, S.R.; Ramesh, N. Effect of nano-CaCO 3 content on flexural and impact properties of PPS/GF ternary composites. Int. J. Eng. Res. Technol. 2012, 1, 1-7.

58. Lopez-Gaxiola, D.L.; Jubinski, M.M.; Keith, J.M.; King, J.A.; Miskioglu, I. Effects of carbon fillers on tensile and flexural properties in polypropylene-based resins. J. Appl. Polym. Sci. 2010, 118, 1620-1633.

59. Díez-Pascual, A.M.; Naffakh, M.; Marco, C.; Ellis, G. Rheological and tribological properties of carbon nanotube/thermoplastic nanocomposites incorporating inorganic fullerene-like $\mathrm{WS}_{2}$ nanoparticles. J. Phys. Chem. B 2012, 116, 7959-7969.

60. Díez-Pascual, A.M.; Naffakh, M.; Marco, C.; Ellis, G. Mechanical and electrical properties of carbon nanotube/polyphenylene sulphide composites incorporating polyetherimide and inorganic fullerene-like $\mathrm{WS}_{2}$ nanoparticles. Compos. Part A 2012, 43, 603-612.

61. Rapoport, L.; Nepomnyashcy, O.; Verdyan, A.; Popovitz-Biro, R.; Volovik, Y.; Ittah, B.; Tenne, R. Polymer nanocomposites with fullerene-like solid lubricant. Adv. Eng. Mater. 2004, 6, 44-48.

62. Friedrich, K.; Schlarb, A.K. Tribology of Polymeric Nanocomposites; Briscoe, B.J., Ed.; Elsevier: Amsterdam, The Netherlands, 2008.

63. Wang, Q.H.; Xu, J.; Shen, W.; Xue, Q. The effect of particle size of nanometer ZrO2 on the tribological behaviour of PEEK. Wear 1997, 209, 316-321.

64. Shwartz, C.J.; Bahadur, S. Studies on the tribological behavior and transfer film-counterface bond strength for polyphenylene sulfide filled with nanoscale alumina particles. Wear 2000, 237, 261-273.

65. Kanny, K.; Jawahar, P.; Moodley, V.K. Mechanical and tribological behavior of clay polypropylene nanocomposites. J. Mater. Sci. 2008, 43, 7230-7238. 


\title{
Continuous Production of IF-WS $\mathbf{2}_{\mathbf{2}}$ Nanoparticles by a Rotary Process
}

\author{
Fang Xu, Nannan Wang, Hong Chang, Yongde Xia and Yanqiu Zhu
}

\begin{abstract}
This manuscript demonstrates the design, modification and initial investigation of a rotary furnace for the manufacturing of inorganic fullerene $\mathrm{WS}_{2}$ nanoparticles. Different preparation methods starting with various precursors have been investigated, of which the gas-solid reaction starting with $\mathrm{WO}_{3}$ nanoparticles was the most efficient technique. Furthermore, the influence of temperature, reaction time, and reaction gases etc. on the synthesis of inorganic fullerene $\mathrm{WS}_{2}$ nanomaterials was investigated, and these parameters were optimised based on combined characterisations using XRD, SEM and TEM. In addition, the furnace was further modified to include a baffled tube, a continuous gas-blow feeding system, and a collection system, in order to improve the batch yield and realise continuous production. This technique has improved the production from less than $1 \mathrm{~g} / \mathrm{batch}$ in a traditional tube furnace to a few tens of $\mathrm{g} / \mathrm{b}$ atch, and could be easily scaled up to industry level production.
\end{abstract}

Reprinted from Inorganics. Cite as: Xu, F.; Wang, N.; Chang, H.; Xia, Y.; Zhu, Y. Continuous Production of IF-WS 2 Nanoparticles by a Rotary Process. Inorganics 2014, 2, 313-333.

\section{Introduction}

Inorganic nanotubes and inorganic fullerene-like (IF) structures of $\mathrm{WS}_{2}$ were first discovered by Tenne et al. in 1992 [1], which has opened a challenging field for the synthesis and applications of numerous such layered structures, such as $\mathrm{WS}_{2}$ [1], $\mathrm{MoS}_{2}$ [2,3], $\mathrm{BN}$ [4], $\mathrm{NiCl}_{2}$ [5], and etc. Various synthesis methods have been reported, such as the microwave treatment of $\mathrm{W}(\mathrm{CO})_{6}$ (Tungsten carbonyl) reacting with $\mathrm{H}_{2} \mathrm{~S}$ (hydrogen sulphide) [6], ultrasonic irradiation of $\mathrm{W}(\mathrm{CO})_{6}$ solution mixed with diphenylmethane and sulphur followed by heating at $800{ }^{\circ} \mathrm{C}$ [6], commercial $\mathrm{WS}_{2}$ activation [7], iodine transport method [8], direct pyrolysis of $\mathrm{WS}_{4}{ }^{2-}$ and CTAB (Cetyltrimethyl Ammonium Bromide) [9], and chemical vapour deposition [10]. Recently, IF-WS 2 nanoparticles and nanotubes have also been obtained from $\mathrm{WCl}_{n}$ and $\mathrm{WO}_{x} \mathrm{Cl}_{y}$ reacting with $\mathrm{H}_{2} \mathrm{~S}$ [11].

These IF-WS 2 and IF-MoS 2 nanomaterials, in addition to their significant mechanical, biocompatible and electronic properties, are excellent solid lubricants [12-20]. Accordingly, the incorporation of these nanomaterials into a proper matrix in composites will lead to new products with hugely improved physical and mechanical properties. Another extraordinary property of $\mathrm{WS}_{2}$ nanostructures is their superb shock absorbing performance [21-23], which suggests an important field of application in lightweight and high performance protective composites [24]. Such applications will obviously demand large amounts of IF-WS2 supply, however their synthesis was only obtained in gram level at the early stage, which was far too less for any practical work. More recently, Tenne's group has produced such IFs in large quantities by using new high tower reactors [25], hence realising a great industrial level success in nanomaterials. Nevertheless, an alternative, simple, versatile and yet effective process for the synthesis of such novel nanomaterials remains highly desirable. 
Therefore, to develop an innovative, simple and scalable technique that is suitable for the continuous manufacture of IF-WS 2 nanomaterials becomes the primary aim of this work. From previous studies [25-27], where a deeper understanding of the formation mechanism of IF-WS2 nanoparticles has been gained, it is found that the key technical barriers for scaling up of the IF-WS2 lie in the powder agglomeration and superficial reaction, which occurs inevitably in a static gas-solid reaction. In order to achieve a large quantity manufacturing, effective measures should be taken to overcome these challenges.

This manuscript describes the design and modification of a rotary furnace, and the initial investigations in scaling up manufacturing of IF-WS 2 nanoparticles using the rotary furnace. Several processes starting with different precursors have been investigated, of which the gas-solid reaction using $\mathrm{WO}_{3}$ nanoparticles as the precursor was the most efficient technique. The influence of temperature, reaction time, precursor types and reaction gases etc. on the synthesis of IF-WS nanomaterials will be optimised. A significantly improved batch yield and a continuous process have been achieved.

\section{Results and Discussion}

\subsection{Design and Modification of the Rotary Furnace}

A novel rotary reactor has been designed for the manufacturing of IF-WS 2 nanomaterials. The rotary reactor was designed based on a conventional tube furnace. The furnace is $1 \mathrm{~m}$ long and has a working temperature up to $1200^{\circ} \mathrm{C}$, which ensures a long enough hot zone for complete reactions at the required temperatures.

Figure 1. Sketch of the rotary furnace. In a traditional static furnace, the $\mathrm{WO}_{x}$ particles stay still in the quartz tube at high temperature $\left(800-900^{\circ} \mathrm{C}\right)$, which leads to the dominance of $2 \mathrm{H}-\mathrm{WS}_{2}$ in the final products; whilst in the present furnace, the quartz working tubes are rotating, forcing the $\mathrm{WO}_{3}$ and formed $\mathrm{WS}_{2}$ nanoparticles to rotate and move during the process, resulting in better separated IF-WS 2 products at minimal agglomeration.

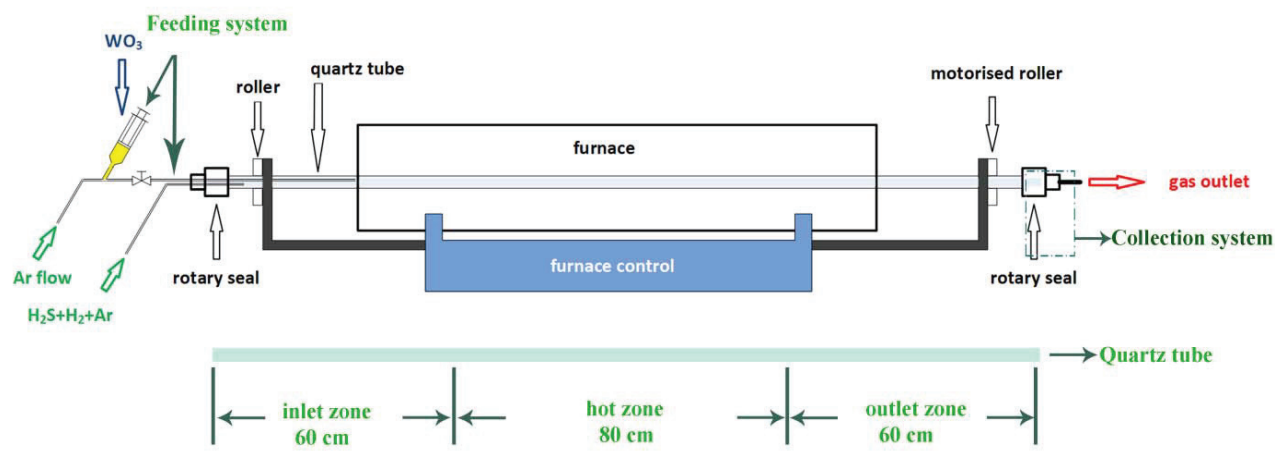

As shown in Figure 1, the rotary furnace consists of several essential parts: a basic tube furnace, a motorised driving and rolling system, an inclining system, a dynamic rotary seal system, a 
continuous feeding system and a collection system. The quartz working tube can be further modified to improve the batch yield. The working tube has inner diameter of $36 \mathrm{~mm}$ and outer diameter of 40 $\mathrm{mm}$. Further modification was realised by adding two small oppositely positioned quartz rod blades of $\varnothing 5 \mathrm{~mm}$ to the inner wall (Figure $\mathrm{S} 1$ ), to provide extra forces driving the movement of powders inside the reactor. This would further eliminate particle agglomerations.

In order to extend the batch process to a continuous production, a continuous feeding system is required. In the current laboratory scale trial, after considering the existing screw feeding and piston feeding mechanisms [28-30], we adopted a simple pump piston feeder, which is more cost-effective. Actually, the current piston feeder under gravitation and gas blow works well in the current set up, since the slight vibration of the reactor helped avoid system blocking and keep a constant feeding. In industrial level, a proper, accurate screw feeder could be used to replace the current feeder. As shown in Figures 1 and S2, the feeder consists of a $20 \mathrm{~mL}$ push pump connected to a "T" junction, acting as the combined inlet blow gas and nanoparticle passage channel. The conceptual rotary furnace enables us to experiment with various parameters for optimal IF-WS2 manufacture. A simple collection system has also been designed, as shown in the dashed box in Figure 1, which makes the process continuous. The detailed schematic drawing of the collection system is shown in Figure S3. The key features of the practically assembled furnace are listed in Table 1.

Table 1. Key parameters of the designed furnace.

\begin{tabular}{clccc}
\hline Temperature & Gas supply & Feeding level & Speed range & Inclination angle \\
\hline $0-1200^{\circ} \mathrm{C}$ & $\mathrm{Ar}, \mathrm{H}_{2} \mathrm{~S}, \mathrm{H}_{2}$ & Up to $100 \mathrm{~g} *$ & $0-350 \mathrm{rpm}$ & -5 to $5^{\circ}$ \\
\hline \multicolumn{5}{c}{ *: depending on numbers of loaded pump container. }
\end{tabular}

\subsection{IF-WS 2 Synthesis by Different Methods}

Different raw materials, including $\mathrm{WO}_{3}$ and $\mathrm{S}$ mixture under $\mathrm{H}_{2}$, ammonium paratungstate (APT) reacting with $\mathrm{H}_{2} \mathrm{~S}$ gas, and $\mathrm{WO}_{3}$ reacting with $\mathrm{H}_{2} \mathrm{~S}$, will be tested in this section.

\subsection{1. $\mathrm{WO}_{3}$ and $\mathrm{S}$ Reaction under $\mathrm{H}_{2}$}

The $\mathrm{WO}_{3}$ precursor exhibits an average particle size of around $60 \mathrm{~nm}$, having a monoclinic $\mathrm{WO}_{3}$ structure (JCPDS No. 43-1035), as shown in Figures S4 and S5.

For the initial trial (experiment $\mathrm{S} 1$, as listed in Table 2), a composite powder of $\mathrm{WO}_{3}$ and $\mathrm{S}$ at a molar ratio of a 1:10 was used as the precursor, and reacted under $\mathrm{Ar} / \mathrm{H}_{2}$ atmosphere at $800{ }^{\circ} \mathrm{C}$, following the procedure described in Experimental Section 3.3.1. SEM images in Figure 2 show that very small nanoparticles and agglomerates were observed, with some nanoplatelets. However, the EDX spectrum shows that although $\mathrm{WS}_{2}$ has formed, $\mathrm{WO}_{x}$ still dominates the products. This is also confirmed by XRD study as shown in Figure 3. In Figure 3, the peaks labelled with a triangle matched well with $\mathrm{WS}_{2}$ (JCPDS No. 84-1398) [26], and all the other peaks labelled with a star could be assigned to $\mathrm{W}_{18} \mathrm{O}_{49}$ and $\mathrm{W}_{20} \mathrm{O}_{58}$. The highest $\mathrm{WS}_{2}$ peak is at 2-theta $14.3^{\circ}$, corresponding to (002). The relatively low intensity of (002) plane for $\mathrm{WS}_{2}$ reveals that only a few $\mathrm{WS}_{2}$ layers have formed from the outside and leaving behind a $\mathrm{WO}_{x}$ core which has been reduced from $\mathrm{WO}_{3}$ to $\mathrm{W}_{18} \mathrm{O}_{49}$ and 
$\mathrm{W}_{20} \mathrm{O}_{58}$. The presence of $\mathrm{W}_{18} \mathrm{O}_{49}$ and $\mathrm{W}_{20} \mathrm{O}_{58}$ is in agreement with previous studies [26,31-33], in which partially reduced $\mathrm{W}_{20} \mathrm{O}_{58}$ and thoroughly reduced $\mathrm{W}_{18} \mathrm{O}_{49}$ were formed during the oxide-to-sulphide conversion from $\mathrm{WO}_{3}$ to IF- $\mathrm{WS}_{2}$. There is no detectable $\mathrm{S}$ left in the products.

Figure 2. SEM images (a-c) and EDX spectrum (d) for products from experiment S1.
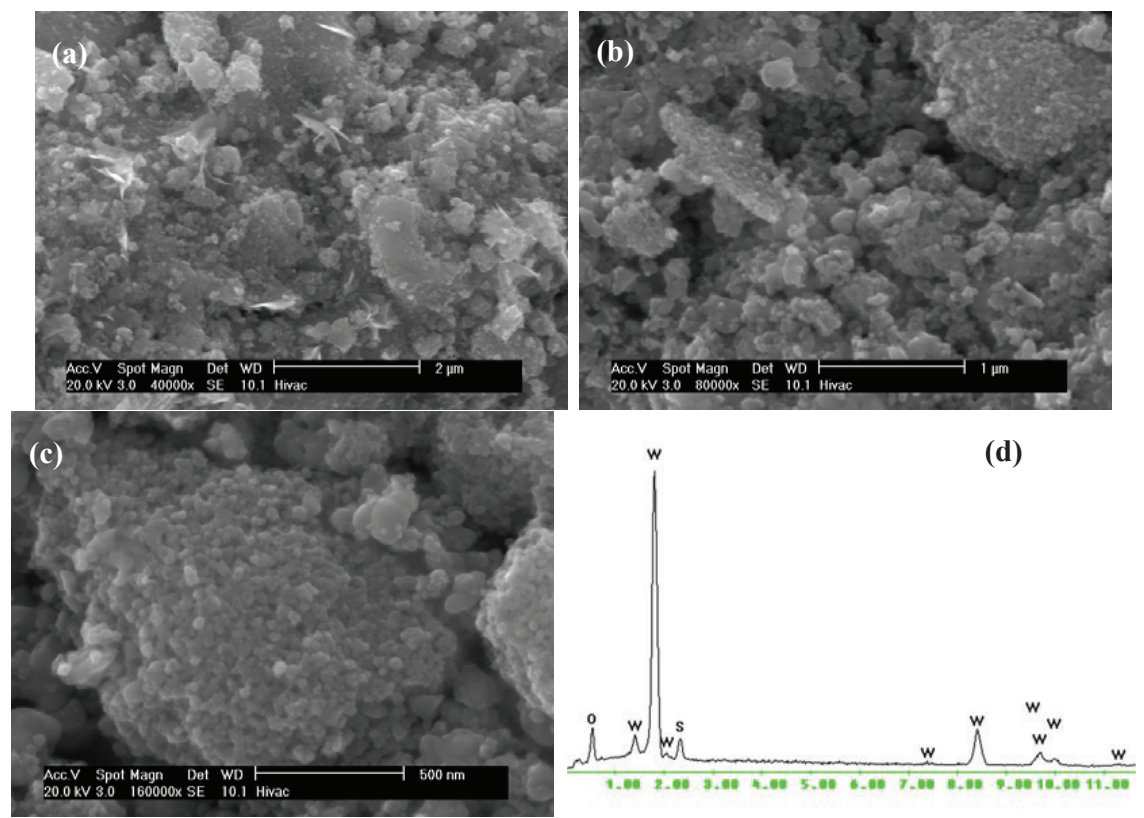

Figure 3. XRD pattern for $\mathrm{WS}_{2}$ from experiment $\mathrm{S} 1$ (reaction of $\mathrm{WO}_{3}, \mathrm{~S}$ and $\mathrm{H}_{2}$ ).

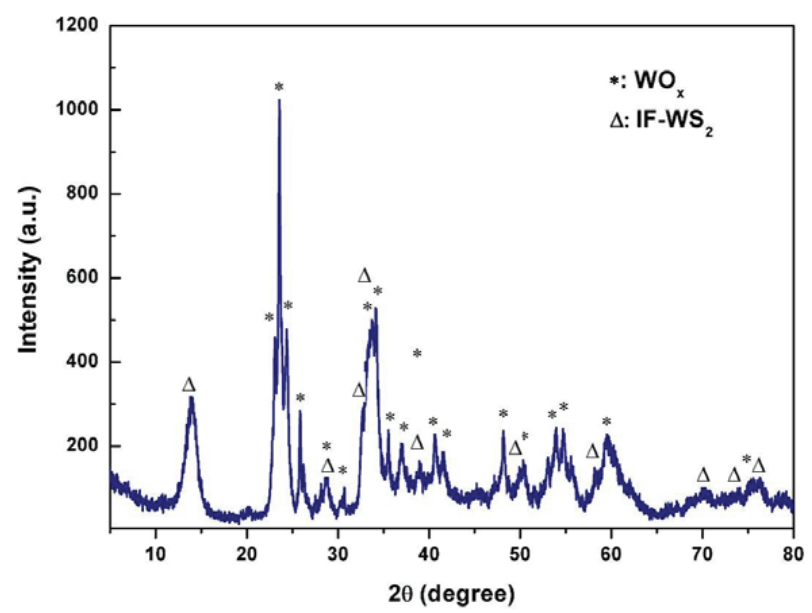

Although a molar ratio of 10:1 for $\mathrm{S}$ and $\mathrm{W}$ had been initially used, it seems that there was not enough $\mathrm{S}$ to react with the reduced $\mathrm{WO}_{x}$ core, because the $\mathrm{S}$ could not stay long enough in the hot zone. As soon as the powders reached the high temperature zone, the $\mathrm{S}$ would be sublimed and blown 
out of the high temperature zone, even under an optimised $\mathrm{Ar} / \mathrm{H}_{2}$ flow rate of $80 \mathrm{~mL} / \mathrm{min}$ [34]. Therefore, the oxide-to-sulphide conversion could not be completed.

There are some similar works that used the same molar ratio of $\mathrm{S}: \mathrm{WO}_{3}=10: 1$ [34], but only achieved a yield of less than $0.1 \mathrm{~g}$ per batch. It is obvious that this method is unsuitable for scaling up, as the product quality depends upon the quantity of $\mathrm{WO}_{3}$ nanoparticles used and it is difficult to increase this for traditional static furnaces. To avoid the $\mathrm{S}$ loss, another batch process has also been reported recently [35], using solid $\mathrm{NaBH}_{4}$ or $\mathrm{LiAlH}_{4}$ as the $\mathrm{H}_{2}$ releasing agent in a sealed ampoules. Again, these processes are not suitable for continuous production.

In this context, a continuous feeding and evaporation of $\mathrm{S}$ was tested, to compensate for the $\mathrm{S}$ loss during the reaction, as shown in Figure 1. The $\mathrm{WO}_{3}$ and $\mathrm{S}$ mixed powder at a molar ratio of 1:10 was fed directly into the hot zone gradually using the pump, at elevated temperature, rather than preplaced inside the quartz tube (experiment S2). The XRD analyses of the powders collected from both the inlet and hot zones have showed that only a few $\mathrm{WS}_{2}$ layers formed and the majority remained as $\mathrm{WO}_{x}$, and $\mathrm{S}$ peaks were also present (peaks labelled with circle); although samples from the hot zone exhibited a more complete oxide-to-sulphide conversion (Figure S6).

Morphologically, the blue powders collected from the inlet zone consist of both very small particles and big aggregates, with some bright $\mathrm{S}$ residues, as shown in Figure 4a,b. At the relatively low temperature inlet zone, $\mathrm{S}$ could not completely react with the reduced $\mathrm{WO}_{x}$, thus only a few layer $\mathrm{WS}_{2}$ formed. For the hot zone samples (Figure $4 \mathrm{c}, \mathrm{d}$ ), some big agglomerates are visible, and they are indeed composed of nanoparticles after ultrasonic treatment.

Figure 4. SEM images of products collected from the inlet zone (a and $\mathbf{b}$ ), and hot-zone (c and d) of experiment S2.
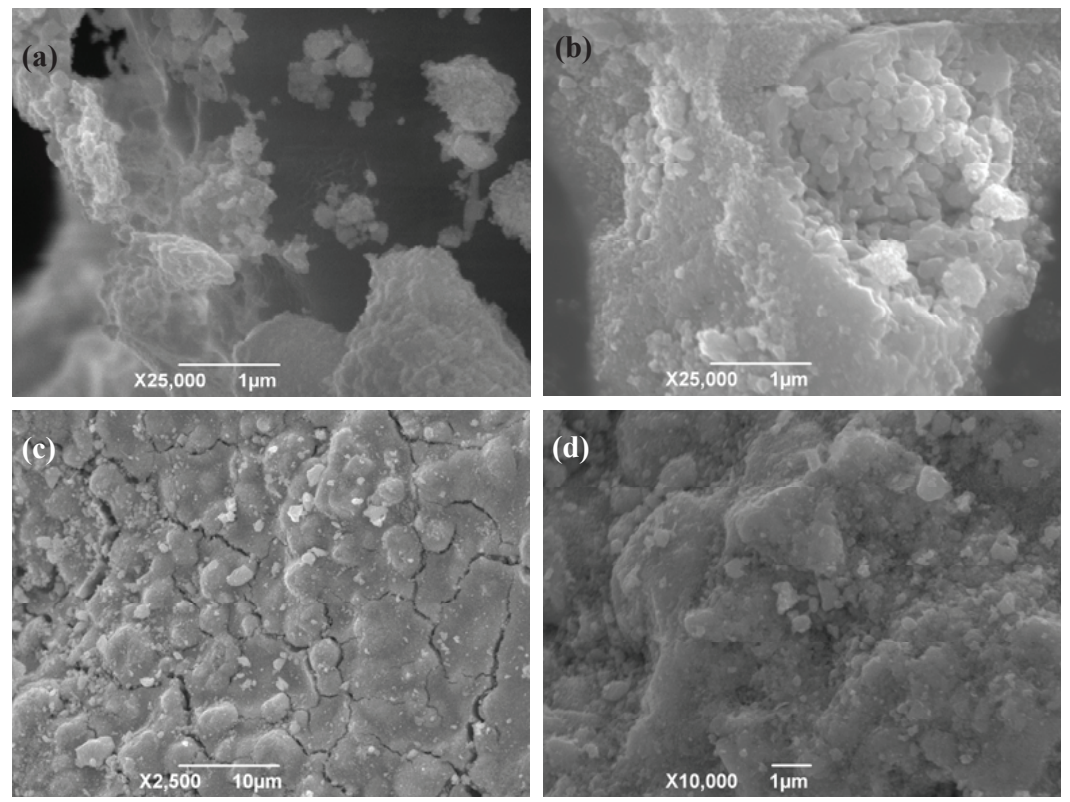
Therefore, although this technique has the potential for scaling up based on the continuous feeding system, the quality of the products is not high enough. Furthermore, when the temperature dropped below its boiling point, the $\mathrm{S}$ vapour could block the outlet gas pipes, which could be a practical issue. The large amounts of $\mathrm{S}$ consumption, although can be recycled, makes this process not cost effective.

\subsubsection{APT as Precursor and $\mathrm{H}_{2} \mathrm{~S}$ as Reaction Gas}

Since $\mathrm{WO}_{3}$ nanoparticles were fabricated by the decomposition of APT, as described in the SI (Supplementary Information 2.2.2, Figures S7 and S8), and were a very valuable precursor for IF-WS 2 nanomaterials in previous two-step studies $[1,25,26,36]$, i.e., first decomposition of APT to form $\mathrm{WO}_{3}$ then via sulphidisation to create IF-WS 2 , it is thus interesting to combine the two steps together in our present set-up. This would be an advantage in terms of energy saving and process efficiency.

The as-received APT particles are crystals of several tens of $\mu \mathrm{m}$ in size, with nano-sized particles attached to the surface of these big crystals. Under high magnification, cracks and sub $\mu \mathrm{m}$ sized particles could be observed at the corners of some damaged crystals, indicating that the APT crystals might be agglomerates of small pieces. Thus, prior to experiment (experiment AHS1), the APT particles were ultrasonic treated, to break the agglomerates into small pieces. The IF-WS 2 particles collected from hot zone are shown in Figure 5. On average, the agglomerates are smaller (Figure 5a), although some bigger aggregates which kept the original APT crystal shape were also observed, as shown Figure 5a,b. Higher magnification study shown in Figure 5c and d reveals the nanostructural feature within the agglomerates, and they are composed of both IF-WS 2 nanoparticles and nanoplatelets, roughly at the same proportion. XRD study (Figure 6) reveals that the hot zone products have very high intensity of $\mathrm{WS}_{2}$ peaks, with only minor peaks of $\mathrm{WO}_{x}$. In contrary, the outlet zone products contain a high portion of $\mathrm{WO}_{x}$, owing to the shorter reaction time than those from the hot zone.

To form $\mathrm{WO}_{x}$ by decomposing APT, the temperature required will be above the melting point of tungsten oxide, e.g., $1200{ }^{\circ} \mathrm{C}$ to form $\mathrm{WO}_{3}$ nanoparticles and $1350{ }^{\circ} \mathrm{C}$ for micro particles [37,38]. This allows for the tungsten oxide vapour to be brought to and deposited in the low temperature area. In the present process, the formed nanoparticles would then play the template role during subsequent sulphidisation, to form the IF-WS 2 . Because of the different temperature requirements for the two steps, the direct IF-WS 2 synthesis using APT to react with $\mathrm{H}_{2} \mathrm{~S}$ seems to be an unsuitable choice for the present furnace. 
Figure 5. SEM images of samples from experiment AHS1, exhibiting the original shape and size of the APT (a and b), and the nanostructural feature within the agglomerates (c and d).
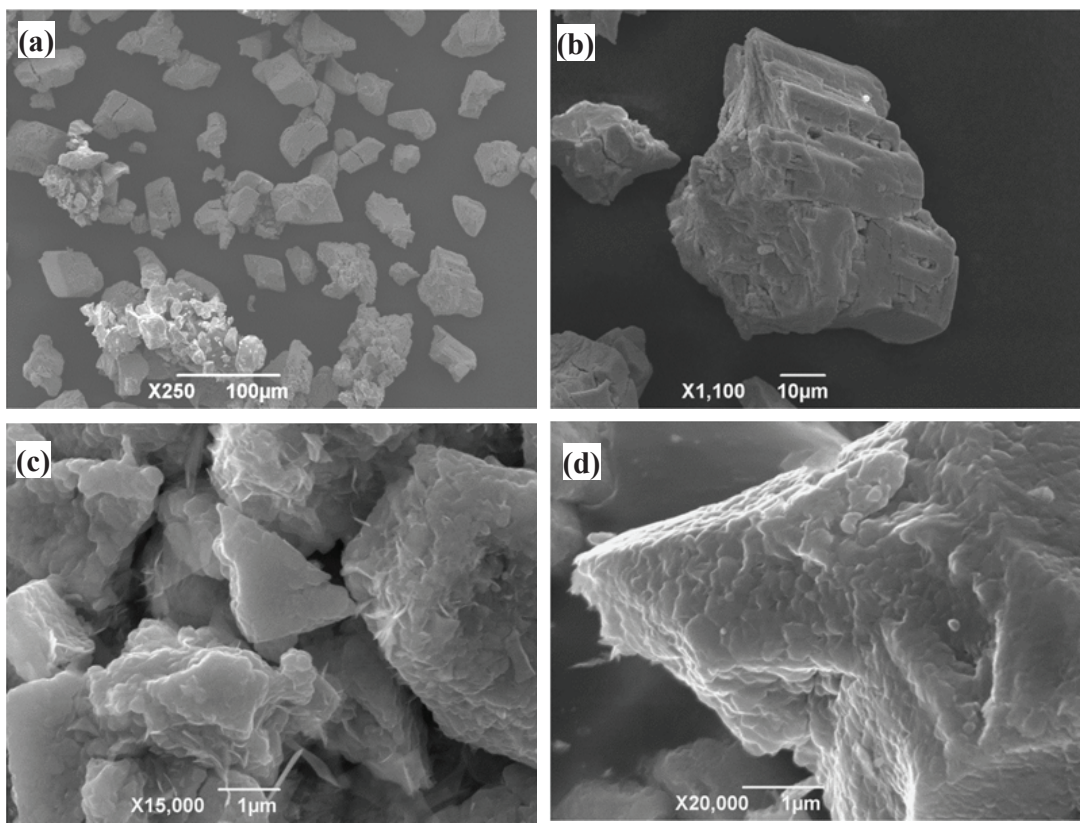

Figure 6. XRD patterns of samples collected from different areas (a, outlet and $\mathbf{b}$, hot zone) in experiment AHS1.

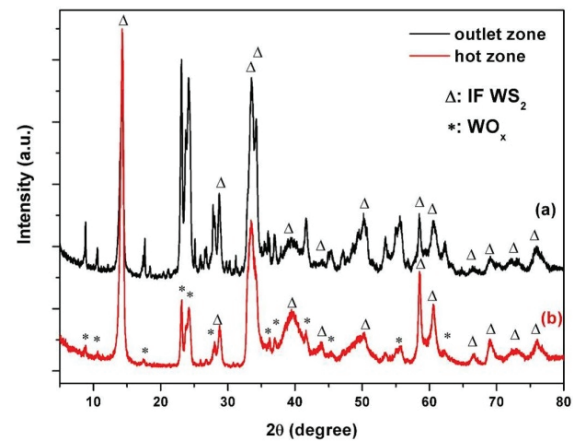

\subsection{3. $\mathrm{WO}_{3}$ and $\mathrm{H}_{2} \mathrm{~S}$ Synthesis of $\mathrm{WS}_{2}$ Nanomaterials}

Figure 7 shows the SEM results of experiment W1. A feature of nanoparticle domination is visible, with big agglomerates (Figure 7a). The semi-spherical IF-WS 2 nanoparticles exhibit different sizes (Figure $7 \mathrm{~b}$ ): the tiny ones have diameters of $<50 \mathrm{~nm}$ which are the same as the $\mathrm{WO}_{3}$ precursor; whilst the big ones are about 100-200 nm, possibly merging from two or more nanoparticles. The presence of nanosheets or nanoplatelets amongst nanoparticles can be seen from Figure 7c (arrowed). 
XRD investigation confirms that the majority of the products are IF-WS 2 nanoparticles, with left-shifted (002) peak and broadened (103) and (105) peaks [3], Figure 7d. Tiny peaks at 23-25 degrees (labelled with a star) can be assigned to $\mathrm{WO}_{x}$ core residue. XRD comparison between the produced IF-WS 2 and the commercial $2 \mathrm{H}-\mathrm{WS}_{2}$ has been presented in the Supplementary Information, as Figure S9, where the differences between XRD pattern of the resulting IF-WS 2 and $2 \mathrm{H}-\mathrm{WS}_{2}$ are discussed in detail.

Figure 7. SEM images (a-c) and XRD pattern (d) of IF-WS 2 synthesised using the rotary process.
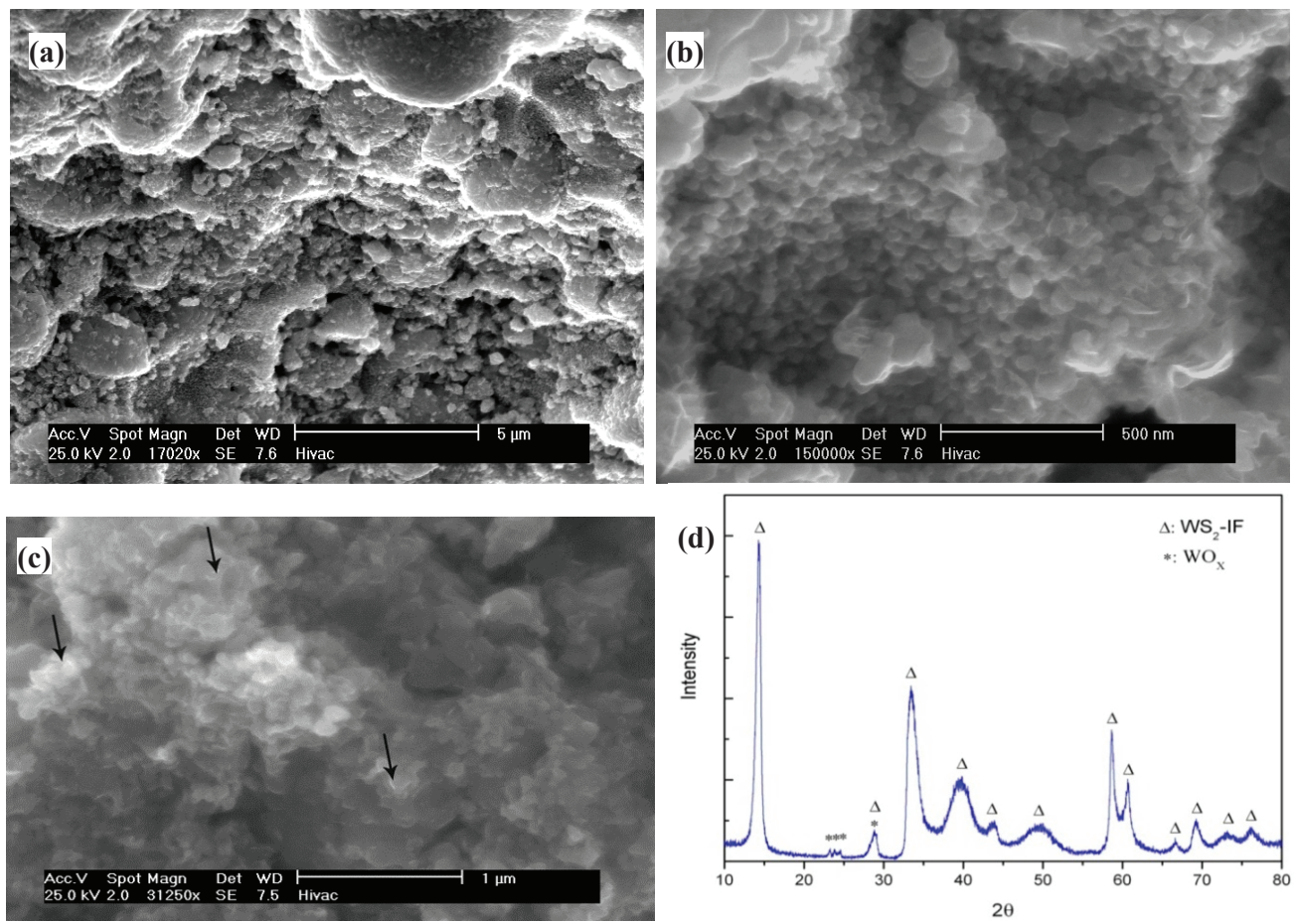

Further TEM examination shows that the sample contains both nanoparticles and nanosheets/nanoplatelets (Figure 8a). Indeed, the IFs exhibit the multi-layered, hollow core characteristics, being the dominant phase. Some particles followed the original shape of their oxide precursor, appearing in a spherical, seamless, and close-caged structure (arrows A); whilst some displayed a peanut-like structure (arrows B) or a long elliptical shape (arrow C). These unusual particles were possibly co-formed from adjacent $\mathrm{WO}_{3}$ nanoparticles that fused together during the heating. The continuous contour of $\mathrm{WS}_{2}$ layers suggests that these particles must have fused together first, then the oxide-to-sulphide conversion occurred. This observation can also explain the different particle sizes observed under SEM, as shown in Figure 8b,c. The products, regardless of their different shapes, possess a hollow core and a generally equal d(002) spacing of $0.62 \mathrm{~nm}$ for IF-WS2. 
Figure 8. TEM images of the $\mathrm{WS}_{2}$ synthesised using the rotary process $(\mathbf{a}-\mathbf{d} ; \mathrm{b}$ is a zoomed-in image of framed area in a).
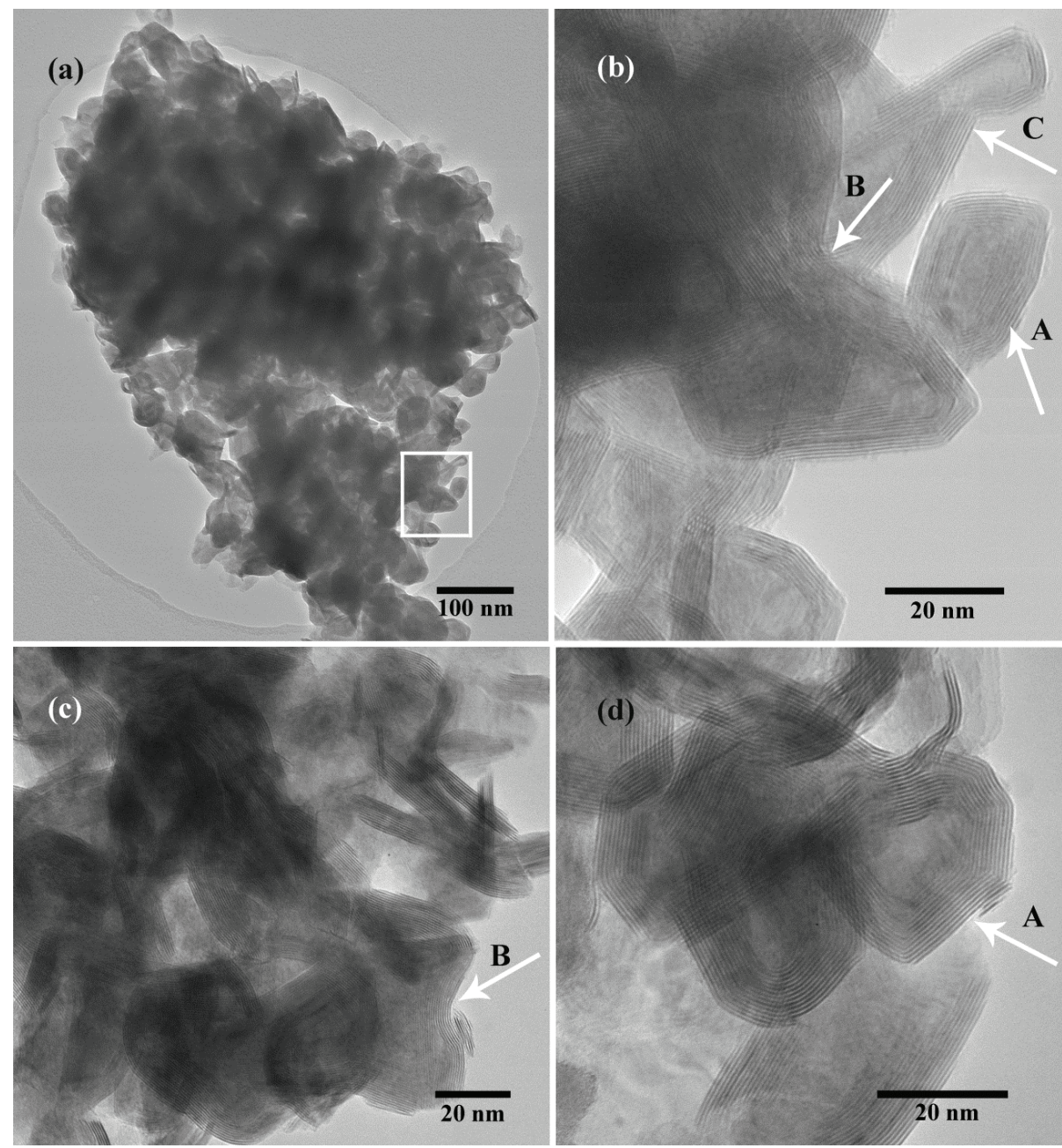

These characterisations of the products have confirmed that IF-WS 2 is the dominant phase in the product with high quality. This shows the great potential for the production of IF-WS 2 at large quantities and high quality, using the present rotary process. Further investigations will focus on quality assessment and quantity improvement.

\subsection{Optimization of $I F-W S_{2}$ Synthesis from $\mathrm{WO}_{3}$ Precursor and $\mathrm{H}_{2} \mathrm{~S}$ Gas}

\subsubsection{Reaction Time}

After applying fixed rotary speed and temperatures, the influence of different reaction times were assessed, as listed in Table 3. Figure 9 shows the resulting products exhibiting similar features as 
described earlier. For the 10 min product (experiment A, Figure 9a), the oxide was just about to be reduced. Only the outer layer finished the oxide-to-sulphide conversion, whilst the inner core remained intact, which is in line with Tenne et al.'s TEM observation [26]. For the 50 min sample (Experiment $B$, Figure 9b), by analysing the intensity changes of the diffraction peaks, it is clear that the oxide particles have mostly converted to IF-WS 2 and there is much less suboxide left in the core. Similarly, after an 80 min reaction, the $\mathrm{WO}_{x}$ peaks at around 23-25 degrees continued to be reduced, and more and more IF-WS 2 layers formed, suggesting an almost complete conversion. Further increase of the reaction time leads to no significant differences in the XRD profiles, and there is almost no peak detected for any tungsten oxide. Thus, a 110 min reaction time is believed to be sufficient for a thorough sulphidisation.

Figure 9. XRD profiles of samples from experiments A-F, demonstrating the effect of different reaction time at $800{ }^{\circ} \mathrm{C}$ from $10-170 \mathrm{~min}$.

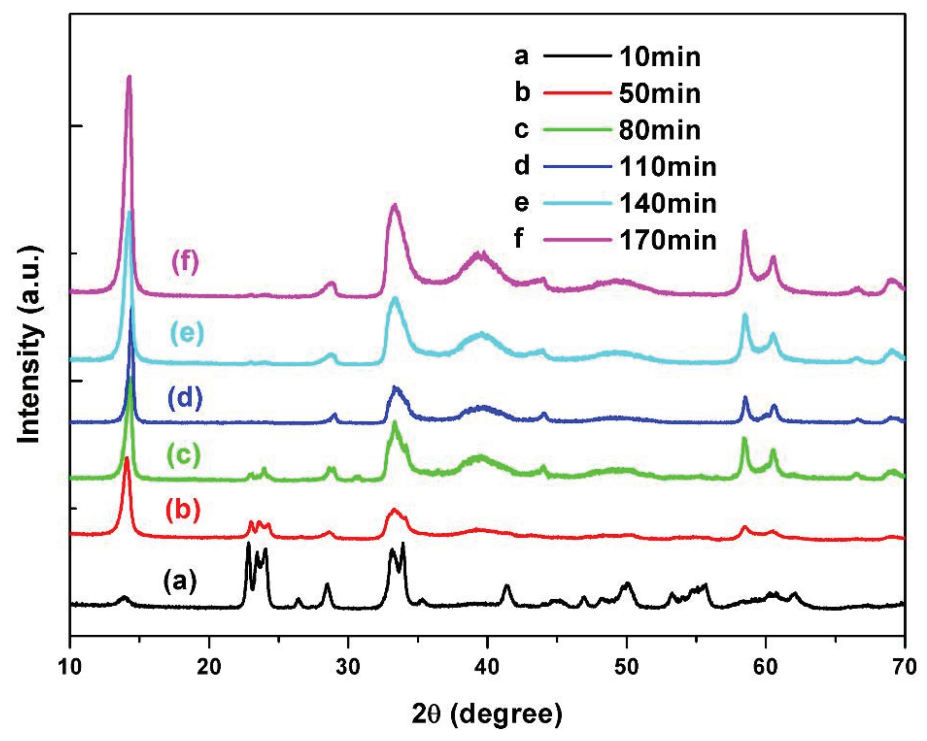

\subsubsection{Reaction Temperatures}

Of the experiments listed in Table 3, batches $\mathrm{C}$ and $\mathrm{G}$ used similar parameters, except for the temperature which was $900{ }^{\circ} \mathrm{C}$ for batch $\mathrm{G}$ but $800{ }^{\circ} \mathrm{C}$ for batch $\mathrm{C}$. From $900{ }^{\circ} \mathrm{C}$, the intensity of the resulting IF-WS 2 (Figure 10) is much higher than that from $800{ }^{\circ} \mathrm{C}$ (Figure 10), confirming more $\mathrm{WS}_{2}$ layers formed at higher temperatures, under the same reaction time of $80 \mathrm{~min}$.

$2 \mathrm{H}-\mathrm{WS}_{2}$ flakes were presented in both samples (Figure 11), however there was much more $2 \mathrm{H}$ $\mathrm{WS}_{2}$ formed in batch $\mathrm{G}$, possibly due to the higher temperature which made agglomeration more severe. The IF-WS 2 particles are also considerably larger in batch G, some of which exhibited a diameter of 300-500 nm, in contrast to 100-200 nm in experiment C. This observation accounted for the extremely high relative intensity of the (002) peak for sample G. 
Figure 10. XRD profiles of samples from batch $\mathrm{C}\left(800^{\circ} \mathrm{C}\right.$, red curve $)$ and $\mathrm{G}\left(900^{\circ} \mathrm{C}\right.$, black curve) and their SEM images.

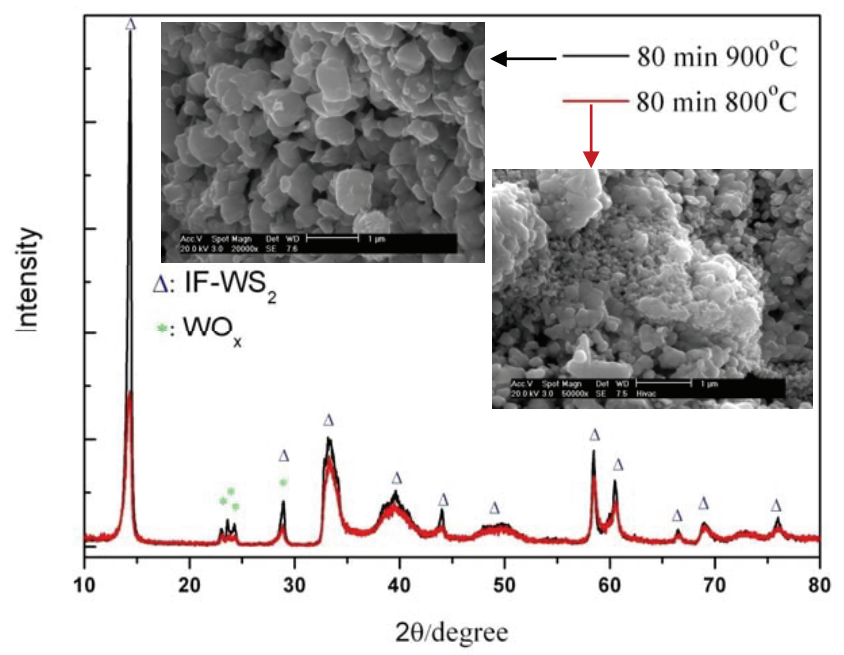

Figure 11. SEM pictures of samples from batch $C(\mathbf{a}-\mathbf{c})$ and batch $G(\mathbf{d}-\mathbf{f})$.
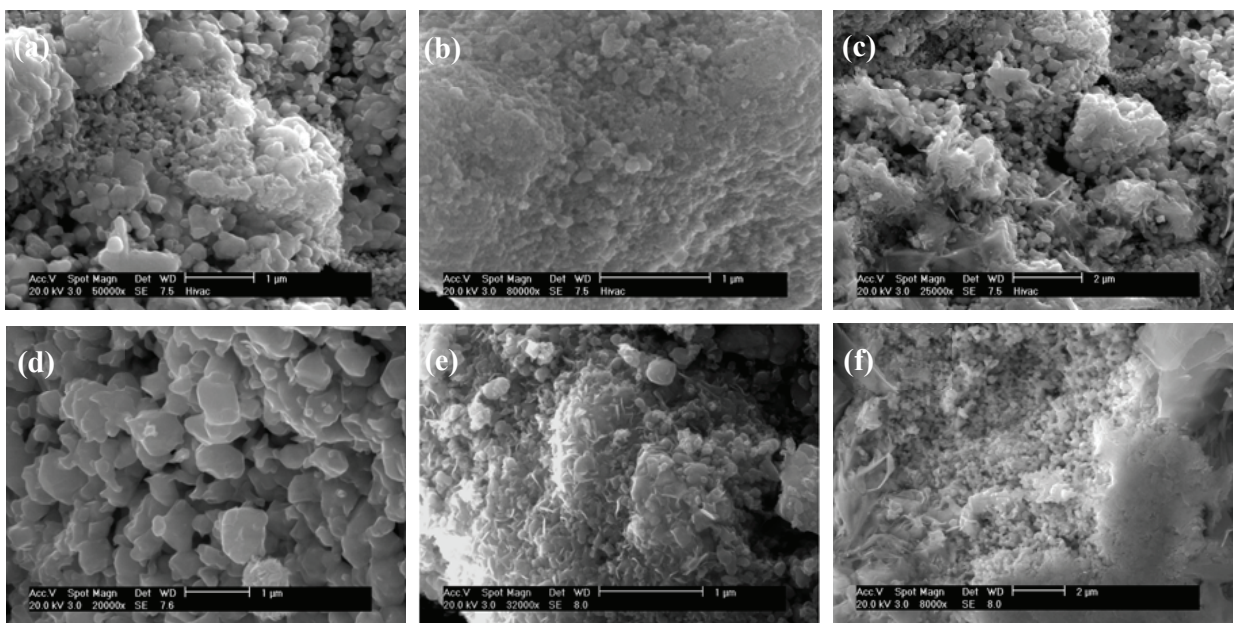

Actually, when compared with other experiments from A-G, the quality of products from batch $\mathrm{G}$ (the highest temperature used, $900{ }^{\circ} \mathrm{C}$ ) are the worst of all, regardless of different reaction times. Thus, temperature is considered to be one of the most significant parameters directly linked with agglomeration in the present process.

\subsubsection{Influence of $\mathrm{H}_{2}$}

$\mathrm{H}_{2}$ was introduced to some experiments (e.g., experiment $\mathrm{H} 1$ ), to promote the $\mathrm{WO}_{x}$ and $\mathrm{H}_{2} \mathrm{~S}$ reaction. In these samples, the majority of the product is IF-WS 2 of less than $100 \mathrm{~nm}$ in size (Figure 12a,b), with 
very few $2 \mathrm{H}-\mathrm{WS}_{2}$ (Figure $12 \mathrm{c}$ ). Rod shaped $\mathrm{WS}_{2}$ were also observed occasionally, as shown in Figure 12d. Indeed, the formation of IF-WS 2 nanotubes was previously reported in the presence of $\mathrm{H}_{2}$ in the reaction gases $[27,39,40]$. The XRD profile looks identical to those without $\mathrm{H}_{2}$. Overall, the results show a positive effect when $\mathrm{H}_{2}$ was added to the reaction gas.

Similarly, many previous studies have indicated that, during the conversion from $\mathrm{WO}_{3}$ to $\mathrm{WS}_{2}$, the $\mathrm{WO}_{3}$ was first reduced to tungsten suboxide such as $\mathrm{W}_{20} \mathrm{O}_{58}, \mathrm{~W}_{18} \mathrm{O}_{49}$ etc. From a localized view, the $\mathrm{S}$ replaced the $\mathrm{O}$ as soon as the reduction of tungsten oxide initiated. From an overall point of view, the reduction and sulfurization processes must have taken place in parallel [26,31-33]. Thus, the addition of $\mathrm{H}_{2}$ into the reaction gases would accelerate the reduction of tungsten oxides and speed up the subsequent sulphidization, thereby leading to prompt formation of the $\mathrm{WS}_{2}$ layers on the oxide surface. Since the early formation of an inert $\mathrm{WS}_{2}$ layer would prohibit the diffusion and agglomeration of neighbouring nanoparticles, $\mathrm{H}_{2}$ introduction helped prevent particles from agglomeration, hence effectively reducing the chance for the formation of $2 \mathrm{H}-\mathrm{WS}_{2}$.

Figure 12. SEM images of samples involving $\mathrm{H}_{2}$ in reaction, showing particles less than $100 \mathrm{~nm}(\mathbf{a}-\mathbf{c})$, and the presence of nanotubes $(\mathbf{d})$.
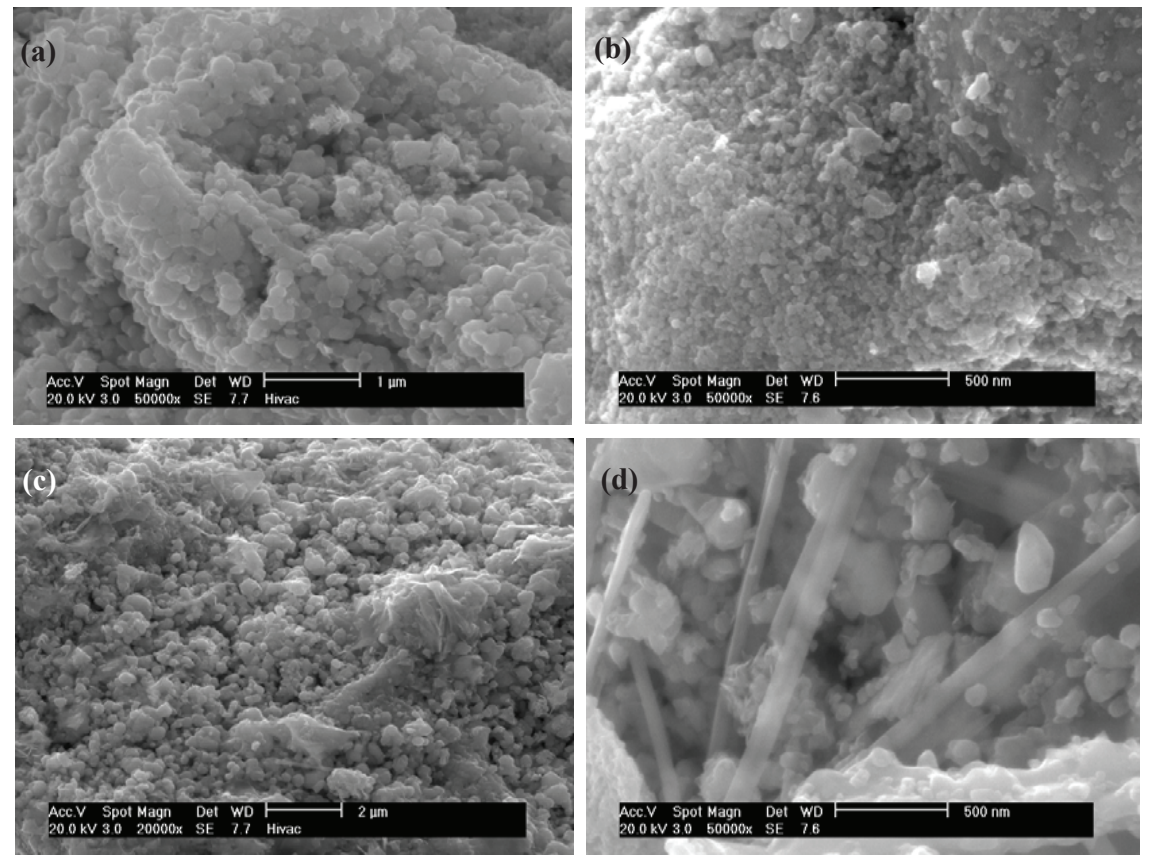

\subsection{Further Refinement and Modification}

\subsubsection{IF-WS 2 Synthesis Using a New Baffled Quartz Tube}

To improve the batch yield, a new baffled quartz tube was designed and adopted for the experiment (Figure S1). Based on previous optimal parameters, changes were made to reflect the 
significantly increased precursor from 6-18 g. For example, the gas rate of $\mathrm{H}_{2}$ and $\mathrm{H}_{2} \mathrm{~S}$, the reaction time and inclination angle etc. were finely adjusted in experiments B1-B3.

For the new baffled tube, a bigger inclination angle was required at the beginning of the experiment in order to drive the particles towards the hot zone, otherwise they would only move around rather than move forward. As soon as all the particles marched into the hot zone, the angle could be decreased to zero for better reaction. In a typical experiment, $18 \mathrm{~g} \mathrm{WO}_{3}$ nanoparticles were used with an initial inclination angle of 5 degrees, and $\mathrm{H}_{2}: \mathrm{H}_{2} \mathrm{~S}$ : Ar ratio of 10:30:160 for the whole process. After the experiment, $15 \mathrm{~g}$ out of the $18 \mathrm{~g}$ were collected from the hot zone. By reducing the $\mathrm{H}_{2}$ gas content in the flow in the later stage of the reaction (experiment B3), the amount of $\mathrm{WS}_{2}$ nanotubes has been dramatically decreased, and they were much shorter (compared to experiments B1 and B2), Figure 13a. The dominant IF-WS 2 nanoparticles appeared to be uniform, fine and spherical, with diameters $<100 \mathrm{~nm}$ (Figure 13b). Their XRD pattern is very promising, with strong $\mathrm{WS}_{2}$ peaks and very tiny $\mathrm{WO}_{x}$ signals, which is again indicative of a good sulphidisation.

Figure 13. SEM images for samples collected from the hot zone of experiment B3, revealing the existence of a small amount of nanotubes (a), and the fine and uniform IF-WS 2 (b).
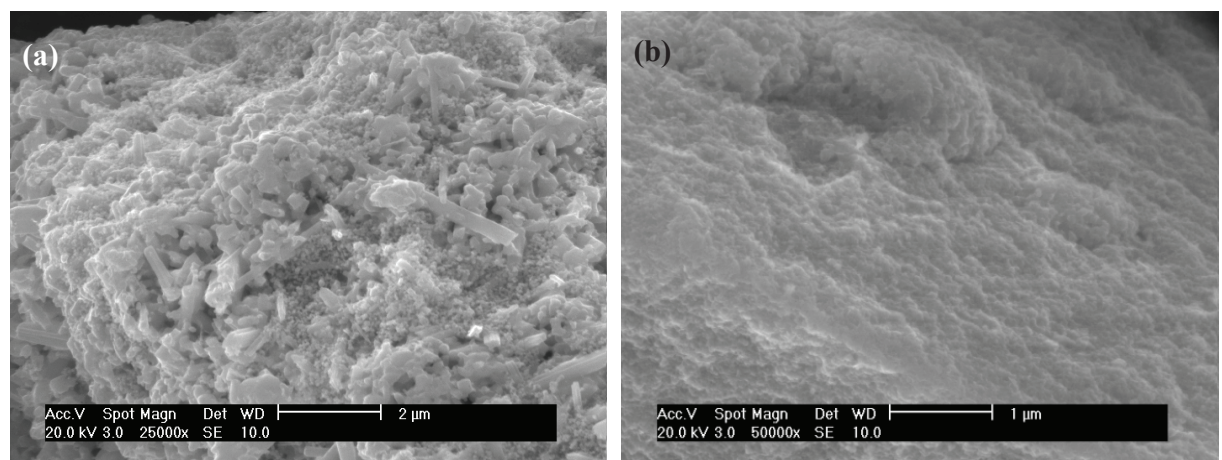

TEM characterisation for sample B3 (Figure 14) further confirm the dominant nature of the IF$\mathrm{WS}_{2}$, with sizes ranging from below $50 \mathrm{~nm}$ to up to $100 \mathrm{~nm}$. Figure $14 \mathrm{~b}$ also demonstrates that some particles coalesced from two or three nanoparticles, exhibiting a peanut shape (arrowed). Those single particles are always $<50 \mathrm{~nm}$. Figure $14 \mathrm{~b}-\mathrm{d}$ show high resolution images of well-crystallised IF-WS 2 particles, which again reveal their typical hollow core and seamless shell layer features. Some particles still possess a residue $\mathrm{WO}_{x}$ core (particle A in Figure 14d). Nevertheless, the new baffled working quartz tube was most promising, enabled a yield improvement from $5 \mathrm{~g}-15 \mathrm{~g}$ per batch whilst successfully maintaining the products quality. However, as the mixing efficiency drops with the filling degree [41], a too high amount of $\mathrm{WO}_{3}$ precursor input would lead to less effective mixing and thus compromise the quality of the final product. Thus, the batch yield and product quality are limited by the quantity of the precursors loaded at the beginning of each batch. Further improvement is still necessary. 
Figure 14. TEM images for samples collected from the hot zone of experiment B3, demonstrating the overall distribution of particle (a) and the multi-layered characteristics of different particles $(\mathbf{b}-\mathbf{d})$.
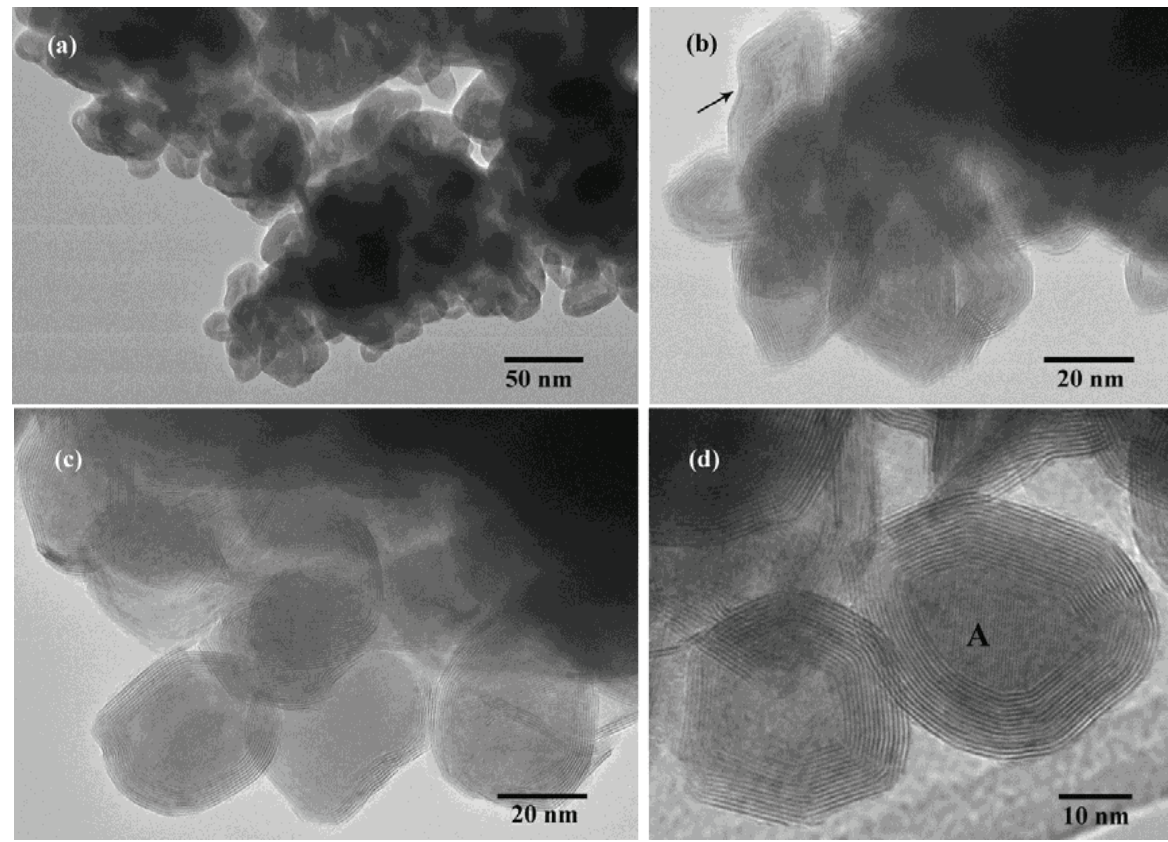

\subsubsection{A Continuous Feeding System}

In this feeding system (Figure 1), the $\mathrm{WO}_{3}$ precursor was first stored in a pump and then introduced to the system by gravity and gas-blow. A single long feeding tube was used to act as the extended pathway for $\mathrm{WO}_{x}$ particles directly blown into the system (as shown in Figures 1 and $\mathrm{S} 2$ ), while the reaction gases were fed in by a separated tube. The new design was tested (FB1), and the result showed a complete conversion of $\mathrm{WO}_{x}$ into $\mathrm{IF}_{-} \mathrm{WS}_{2}$ for particles from the hot zone (Figure $\mathrm{S} 10)$. The products were quite uniform, with sizes no more than $100 \mathrm{~nm}$ (Figure S11).

The process was further modified to simulate a real, continuous one, by immediately replacing the empty pump with a full one (FB2). All other experimental parameters made no alteration to FB1, except for the longer feeding time. In this case, around $50 \mathrm{~g}$ samples were collected from the hot zone. TEM images of the hot zone sample from FB2 were shown in Figure 15. Majority nanoparticles of around 50 $\mathrm{nm}$ in size are displayed in Figure 15a,c, some from $20 \mathrm{~nm}$ to almost $100 \mathrm{~nm}$ are exhibited in Figure 15b. A typical IF-WS 2 particle with a hollow core and around 15 seamless layers is shown in Figure 15d.

To summarise, the continuous feeding system has been proven a success and has improved the yield of IF-WS $\mathrm{W}_{2}$ to several tens of grams per batch, without having an obvious compromise on quality. Technically, the feeding of precursor could be simply continued by reloading; however, a longer reaction time would be required. In an industry environment, where a proper sample collection system is easily available, an automated reaction would not be a limitation. This reactor is easily 
adaptable as a whole continuous rotary process for the scaling up production of IF-WS 2 nanoparticles, using a proper metallic working tube.

Figure 15. TEM images for particles collected from the hot zone from experiment FB2, showing overall uniformity of particles (a and $\mathbf{c}$ ) and detailed closed-cage feature under high resolution (b and $\mathbf{d})$.
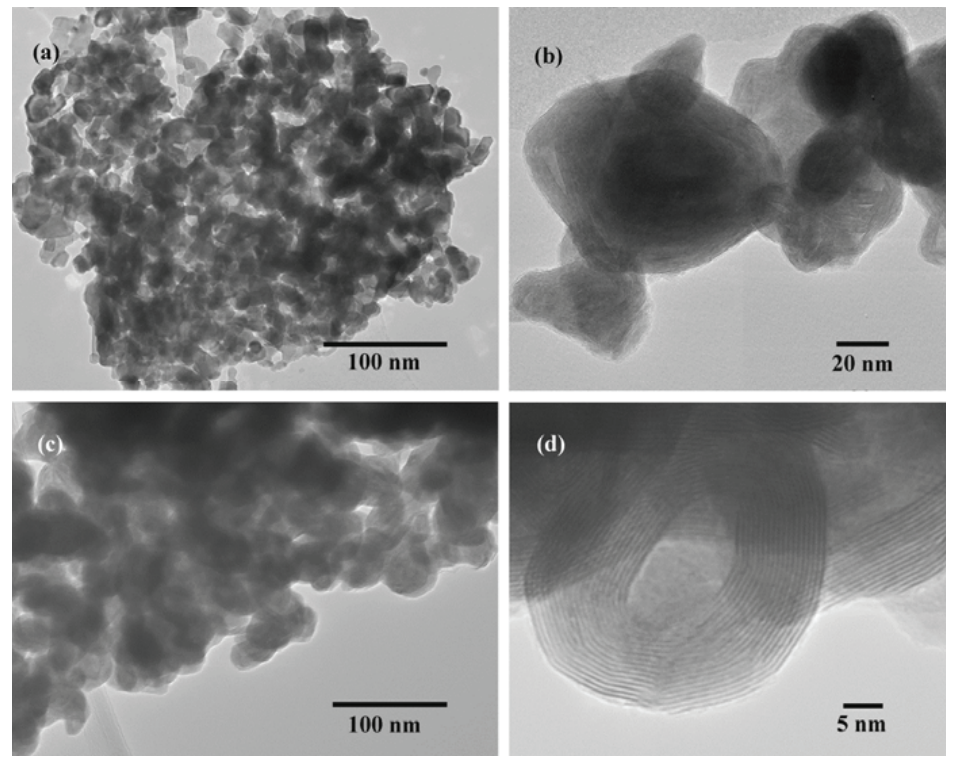

\section{Experimental Section}

\subsection{Materials}

Commercial tungsten trioxide powder used as precursor for IF-WS 2 nanomaterials was monoclinic $\mathrm{WO}_{3}$ powder (light yellow, with size $<100 \mathrm{~nm}$, purity 99\%, Sigma-Aldrich, UK). Ammonium paratungstate (APT, $\left.\left(\mathrm{NH}_{4}\right)_{10} \mathrm{H}_{2}\left(\mathrm{~W}_{2} \mathrm{O}_{7}\right) 6 \cdot x \mathrm{H}_{2} \mathrm{O}, 99.99 \%\right)$ particles used to produce $\mathrm{WO}_{x}$ nanoparticles were bought from Sigma-Aldrich, UK. Sulphur (S, sublimed, 100 mesh, 99.5\%) was purchased from Cole-Parmer, UK. For comparison with the produced IF-WS 2 nanoparticles, $2 \mathrm{H}-\mathrm{WS}_{2}$ platelets (powder, $2 \mu \mathrm{m}, 99 \%$ ) were also bought from Sigma-Aldrich, UK.

\subsection{Experimental Set-Up}

Based on the furnace shown in Figure 1, the parameters for the experiments were optimized through a series of experiments, such as different reaction temperatures, reaction time, reaction gas atmosphere, modification of working tube, and a new feeding system. The optimization experiments were carried out without the feeding system, unless specifically stated. The products were collected from different areas separately, where the central $80 \mathrm{~cm}$ part of the quartz tube in the heated zone was defined as hot zone, the $60 \mathrm{~cm}$ part close to the gas inlet end was named as the inlet zone, and 
similarly the outlet zone, as also shown in Figure 1. Note that the reaction time could be varied mostly through the inclination of the quartz reactor, and the motor could drive the working quartz tube both clockwise and anti-clockwise. In such a way, the particles could be moved either backwards or forwards as required, to achieve sufficiently long reaction time.

The morphology and chemical composition of the as-produced IF-WS 2 nanoparticles were analysed by XRD, SEM and TEM. The TEM samples were prepared via an ultrasonic treatment of the nanoparticles (randomly collected from hot zone) for $5 \mathrm{~min}$ in acetone, and the resultant uniform suspension was pipetted onto a holey carbon coated copper grid (300 mesh, Agar).

\subsection{IF-WS 2 Synthesis by Different Methods}

\subsubsection{Synthesis of IF-WS $\mathrm{W}_{2}$ Nanoparticles from Mixed $\mathrm{WO}_{3}$ and $\mathrm{S}$ Powder with $\mathrm{H}_{2}$}

Two typical types of experiment were carried out to synthesize the IF-WS2, starting from mixed $\mathrm{WO}_{3}$ and $\mathrm{S}$ powder with $\mathrm{H}_{2}$ (experiments $\mathrm{S} 1$ and $\mathrm{S} 2$ ). In experiment $\mathrm{S} 1$, around $6 \mathrm{~g} \mathrm{WO}_{3}$ and $\mathrm{S}$ composite powder at a molar ratio of 1:10 was placed into the inlet area. Ar gas was used to purge the quartz tube at $50 \mathrm{~mL} / \mathrm{min}$ for $30 \mathrm{~min}$, after the system was properly assembled and sealed. The working tube started rotating from $600{ }^{\circ} \mathrm{C}$, at which point $\mathrm{H}_{2}(80 \mathrm{~mL} / \mathrm{min})$ and $\operatorname{Ar}(30 \mathrm{~mL} / \mathrm{min})$ gases were also introduced. The reaction at $800{ }^{\circ} \mathrm{C}$ lasted for $1 \mathrm{~h}$. Similarly, experiment S2 was carried out by adopting a continuous $\mathrm{S}$ feeding system.

\subsubsection{Synthesis of IF-WS 2 Nanoparticles from APT Precursor with $\mathrm{H}_{2} \mathrm{~S}$ Gas}

Six g APT were ultrasonically treated and dried before being placed into the centre of the quartz tube for the experiment (AHS1). Ar $(50 \mathrm{~mL} / \mathrm{min})$ was flushed for $30 \mathrm{~min}$ before the furnace was switched on. $\mathrm{H}_{2} \mathrm{~S}$ flowing was started from $550{ }^{\circ} \mathrm{C}$ till the end of the process, at a rate of $12 \mathrm{~mL} / \mathrm{min}$. The whole reaction lasted for $2 \mathrm{~h}$ at $800^{\circ} \mathrm{C}$, at a fixed Ar flow rate of $100 \mathrm{~mL} / \mathrm{min}$.

Except for experiments on various reaction times (summarised separately), all other experimental parameters are summarised in Table 2.

Table 2. Example parameters used for the synthesis of IF-WS2.

\begin{tabular}{ccccc}
\hline & $\begin{array}{c}\text { Precursor put in } \\
(\mathbf{g})\end{array}$ & $\begin{array}{c}\text { Gas feed Rate } \\
\left(\mathbf{H}_{\mathbf{2}}: \mathbf{H}_{\mathbf{2}} \mathbf{S}: \mathbf{A r}\right)(\mathbf{m L} / \mathbf{m i n})\end{array}$ & $\begin{array}{c}\text { Reaction time } \\
(\mathbf{m i n})\end{array}$ & $\begin{array}{c}\text { Reaction } \\
\text { temperature }\left({ }^{\circ} \mathbf{C}\right)\end{array}$ \\
\hline S1 & 6 & $80: 0: 30$ & 60 & 800 \\
\hline S2 & 12 & $80: 0: 30$ & 120 & 800 \\
\hline AHS1 & 6 & $0: 12: 100$ & 120 & 800 \\
\hline W1 & 6 & $0: 12: 100$ & 90 & 800 \\
\hline B1 & 6 & $8: 12: 100$ & 90 & 800 \\
\hline B2 & 18 & $10: 30: 160$ & 120 & 800 \\
\hline B3 & 18 & $20: 30: 100$ & 120 & 800 \\
\hline FB1 & 18 & $20: 30: 100$ & 120 & 800 \\
\hline FB2 & 50 & $\begin{array}{c}50: 30: 100 \\
0: 25: 100\end{array}$ & 180 & 800 \\
\hline
\end{tabular}




\subsubsection{Synthesis of IF-WS 2 Nanoparticles $\mathrm{WO}_{3}$ and $\mathrm{H}_{2} \mathrm{~S}$ Gas}

The synthesis of $\mathrm{WS}_{2}$ nanomaterials from reaction of $\mathrm{WO}_{3}$ and $\mathrm{H}_{2} \mathrm{~S}$ gas was similar to that from $\mathrm{WO}_{3}$ and $\mathrm{S}$ (Section 3.3.1), except for the $\mathrm{H}_{2}$ gas being replaced by $\mathrm{H}_{2} \mathrm{~S}$. The first trial was named experiment W1 (Table 2).

Table 3. Example parameters used for the synthesis of IF-WS 2 with varying reaction times.

\begin{tabular}{ccccc}
\hline & WO $_{3}$ Precursor $(\mathrm{g})$ & $\begin{array}{c}\text { Gas feed rate }\left(\mathbf{H}_{\mathbf{2}} \mathbf{S}: \mathbf{A r}\right) \\
(\mathbf{m L} / \mathbf{m i n})\end{array}$ & $\begin{array}{c}\text { Reaction time } \\
(\mathbf{m i n})\end{array}$ & $\begin{array}{c}\text { Reaction temperature } \\
\left({ }^{\circ} \mathbf{C}\right)\end{array}$ \\
\hline $\mathrm{A}$ & 6 & $12: 100$ & 10 & 800 \\
$\mathrm{~B}$ & 6 & $12: 100$ & 50 & 800 \\
$\mathrm{C}$ & 6 & $12: 100$ & 80 & 800 \\
$\mathrm{D}$ & 6 & $12: 100$ & 110 & 800 \\
$\mathrm{E}$ & 6 & $12: 100$ & 140 & 800 \\
$\mathrm{~F}$ & 6 & $12: 100$ & 170 & 800 \\
$\mathrm{G}$ & 6 & $12: 100$ & 80 & 900 \\
\hline
\end{tabular}

Table 3 summarises the parameters used for a series of experiment at a fixed rotation speed of $140 \mathrm{rpm}$, with reaction time varying from 10-170 min.

\section{Conclusions}

Based on the built rotary system, the large scale manufacture of IF-WS 2 nanoparticles has been realised. Several synthesis methods have been studied, and the process starting with $\mathrm{WO}_{3}$ precursor and $\mathrm{H}_{2} \mathrm{~S}$ reaction gas is most successful. Systematic studies have been carried out to optimise the parameters for IF-WS 2 nanoparticle production, including precursor types, reaction temperatures, reaction time and reaction gases. Further refinements by modifying the working quartz tube and the feeding system have made the continuous manufacturing possible. This new technique, as a simple alternative to the fluidised bed method, has improved the yield of IF production from less than $1 \mathrm{~g} / \mathrm{batch}$ using a traditional tube furnace to a few tens of $\mathrm{g} / \mathrm{batch}$. This process is easily scalable to industry production level, by incorporating existing equipment.

\section{Supporting Information}

\section{Results and Discussion}

\subsection{Design and Modification of a Rotary Furnace}

The quartz tube modification is shown in Figure S1. Two small quartz rod blades of Ø5 mm were oppositely attached to quartz tube. 
Figure S1. Image (left) and sketch (right) of a new tube with baffles inside, created by adding two quartz rods inside the normal quartz tube.
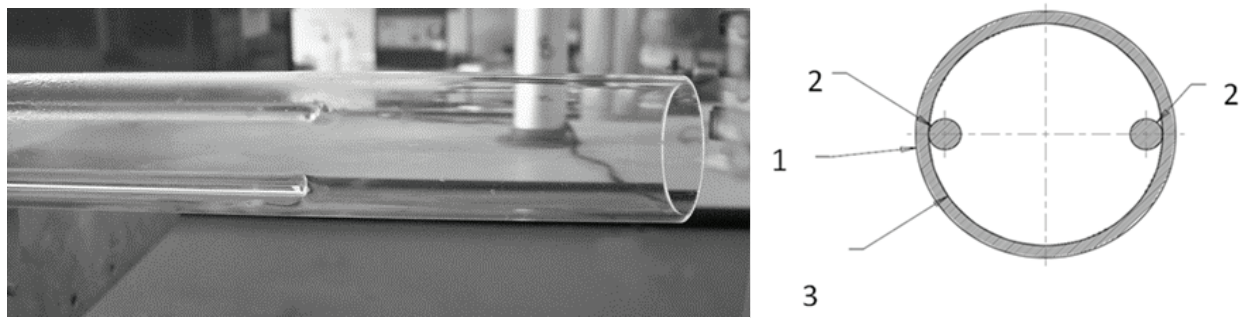

In order to move the batch process to a continuous production, a continuous feeding system was designed using a $20 \mathrm{~mL}$ syringe piston feeder which was connected to a push type ' $\mathrm{T}$ ' junction, as shown in Figure S2.

Figure S2. Sketch (a) and picture (b) of a new gas blow feeding system, to realise continuous feeding.
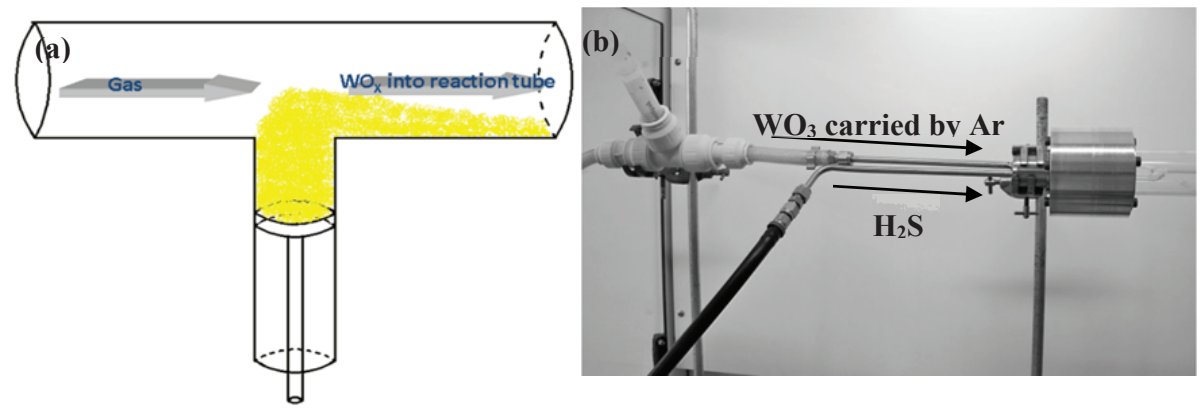

Figure S3. Sketch of the collection system, where (1) quartz tube and (2) housing are rotating while all other parts are kept still during action.

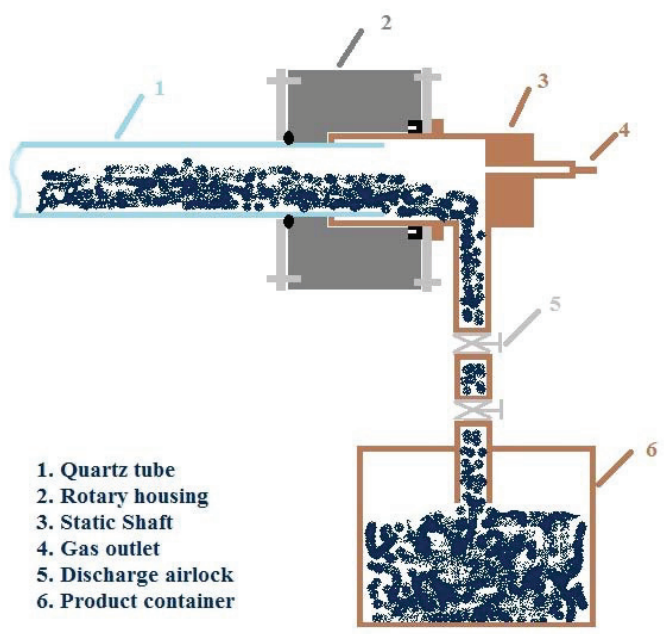


The collection system makes the process a real continuous one, as shown in Figure S3. During the process, the quartz tube (1) and housing were driven to rotate by the motor, while the shaft (3) was kept static, as well as the gas outlet (4) and collection outlet (5 and 6) attached to it. Note that the product discharge outlet allows for easy isolation to collect samples for quality control during the manufacturing.

\subsection{IF-WS 2 Synthesis by Different Methods}

\subsubsection{Mixed $\mathrm{WO}_{3}$ and $\mathrm{S}$ with $\mathrm{H}_{2}$}

The SEM images of the as-received $\mathrm{WO}_{3}$ precursor have been displayed in Figure S4; and the average particles size was about $60 \mathrm{~nm}$, estimated using Derby-Scherrer Equation. The XRD pattern shown in Figure S5 matches very well with the monoclinic $\mathrm{WO}_{3}$ (JCPDS No. 43-1035).

Figure S4. SEM images of the as-received $\mathrm{WO}_{3}$ nanoparticles $(\sim 60 \mathrm{~nm}$ in size $)$.
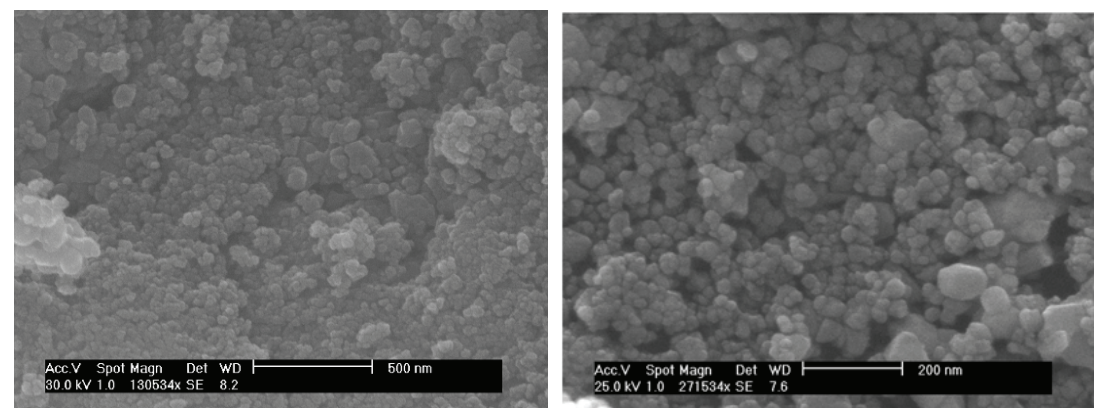

Figure S5. XRD pattern of the as-received $\mathrm{WO}_{3}$ precursor.

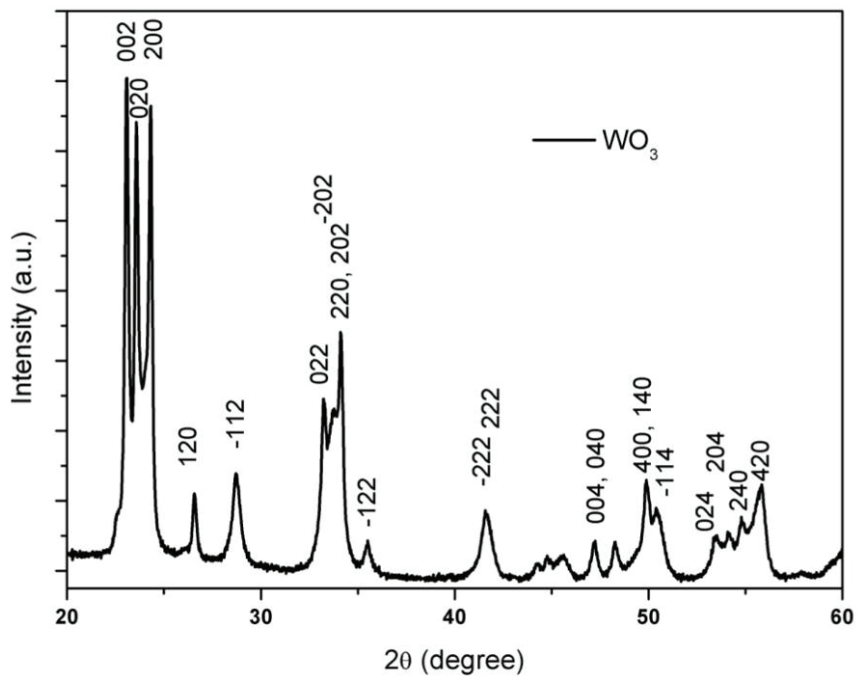


2.2.1. $\mathrm{WO}_{3}$ and $\mathrm{S}$ reaction under $\mathrm{H}_{2}$

The XRD patterns of Sample S2 from both the inlet and the hot zones are shown in Figure S6. Only a few $\mathrm{WS}_{2}$ layers may have formed, as the majority was $\mathrm{WO}_{x}$, along with $\mathrm{S}$ peaks (labelled with circle), although the hot zone samples exhibited a more complete oxide-to-sulphide conversion.

Figure S6. XRD patterns of Sample S2. (a) from the inlet zone and (b) from the central hot zone.

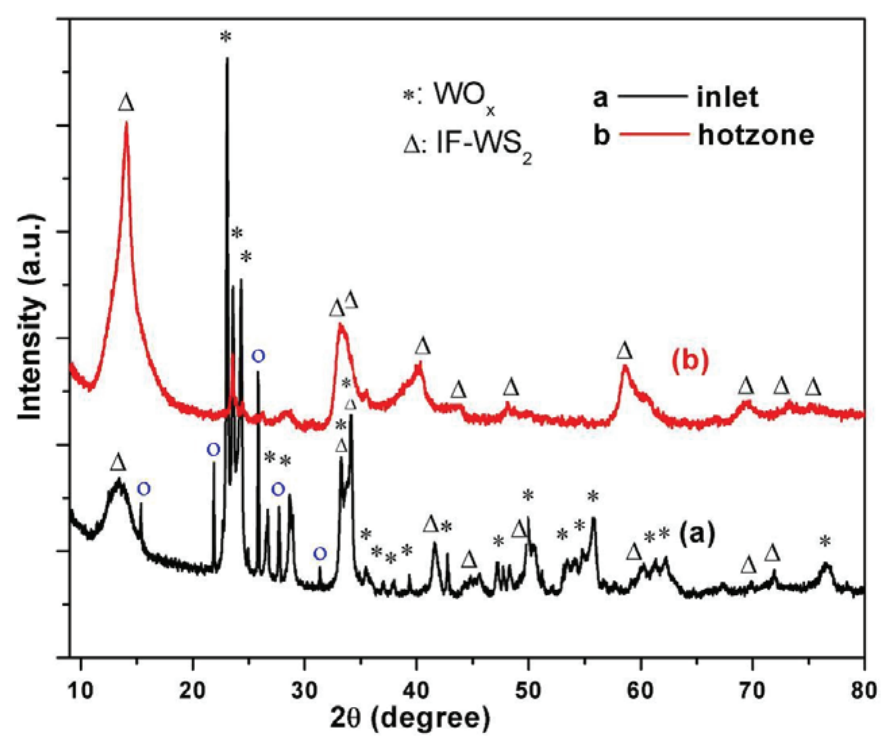

2.2.2. APT as Precursor and $\mathrm{H}_{2} \mathrm{~S}$ as Reaction Gas-Production of $\mathrm{WO}_{x}$ Nanoparticles from APT

$\mathrm{WO}_{x}$ nanoparticles, as an important precursor for IF-WS 2 nanoparticles, have been produced by the decomposition of APT at a high temperature furnace. At high temperature, the following reaction took place: $\left(\mathrm{NH}_{4}\right)_{10}\left[\mathrm{H}_{2} \mathrm{~W}_{12} \mathrm{O}_{42}\right] 4 \mathrm{H}_{2} \mathrm{O} \rightarrow 12 \mathrm{WO}_{3}+10 \mathrm{NH}_{3}+11 \mathrm{H}_{2} \mathrm{O}$.

In fact, the APT started to decompose into $\mathrm{WO}_{3}$ at $500{ }^{\circ} \mathrm{C}$. The $\mathrm{WO}_{3}$ then started to melt and vaporise in the central zone where temperature reached $1200{ }^{\circ} \mathrm{C}$ and $1250{ }^{\circ} \mathrm{C}$ respectively, and the $\mathrm{WO}_{x}$ vapour would be brought downstream by the Ar flow and deposit at the inner wall of the quartz tube.

After optimization of the parameters, very fine nanoparticles with high batch yield were produced at $1350{ }^{\circ} \mathrm{C}$, with long quartz tube $(1.5 \mathrm{~m})$ and high gas flow rate $(6 \mathrm{~L} / \mathrm{min})$. The results were shown in Figure S7a-c. Both spherical and polyhedral particles are presented in all images, with uniform size less than $100 \mathrm{~nm}$, mostly around $50 \mathrm{~nm}$. XRD pattern in Figure S7d matches very well with monoclinic $\mathrm{WO}_{3}$ (JCPDS No. 43-1035), with the strongest peaks appear at 23-25 degree. 
Figure S7. SEM images (a-c), and XRD pattern (d) of tungsten oxide nanoparticle $\left(1350^{\circ} \mathrm{C}, 6 \mathrm{~L} / \mathrm{min}, 30 \mathrm{~min}, 1.5 \mathrm{~m}\right)$.

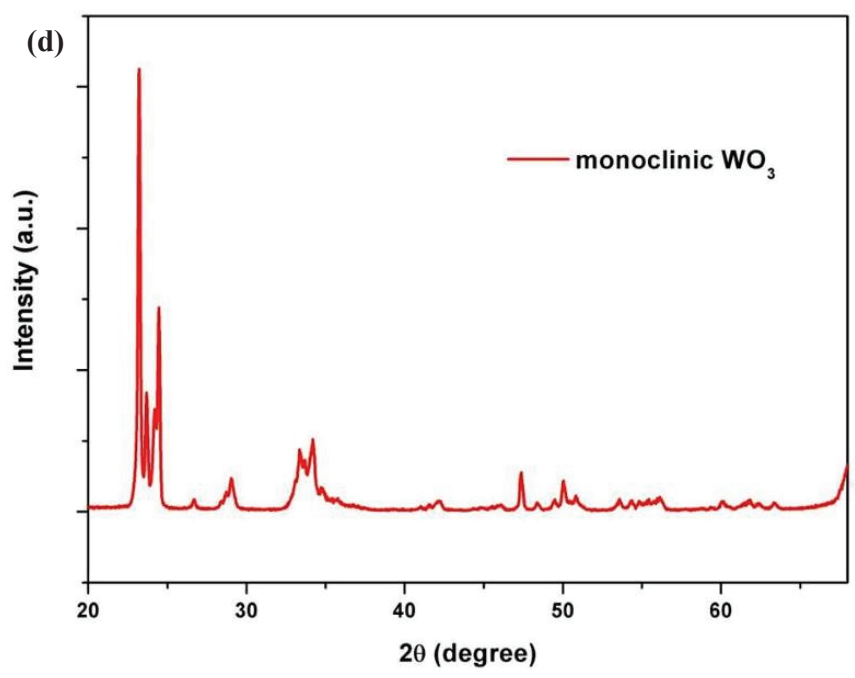

Figure S8. TEM images of $\mathrm{WO}_{3}$ from decomposition of APT $(1350,1.5 \mathrm{~m}, 6 \mathrm{~L} / \mathrm{min}$, $30 \mathrm{~min})$.
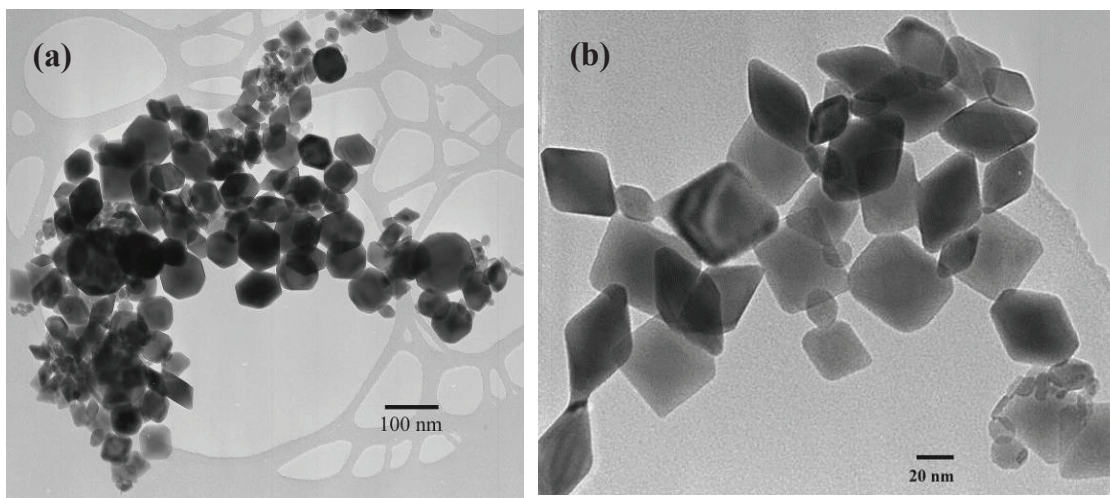

Figure $\mathrm{S} 8$ shows the TEM images of $\mathrm{WO}_{3}$ nanoparticles from the decomposition of APT. The majority of nanoparticles are of a polyhedral shape, around $50 \mathrm{~nm}$.

\subsection{3. $\mathrm{WO}_{3}$ and $\mathrm{H}_{2} \mathrm{~S}$ Synthesis of $\mathrm{WS}_{2}$ Nanomaterials}

A comparison of the XRD profiles of the commercial $2 \mathrm{H}-\mathrm{WS}_{2}$ and the present IF-WS 2 samples is shown in Figure S9. Both patterns showed peaks at very similar positions. The peaks of IF-WS 2 were assigned according to $2 \mathrm{H}-\mathrm{WS}_{2}$ (JCPDS No. 84-1398), as no standard XRD pattern is available for IF-WS 2 [1]. These are the typical peaks at $2 \theta$ angles at: 14.364 (002), $28.959(004), 32.769$ (100), 33.587 (101), 35.943 (102), 39.599 (103), 44.055 (006), 44.289 (104), 49.798 (105), 55.977 (106), 57.495 (110), 60.010 (008), 60.573 (112), 62.746 (107), 69.169 (201), 70.080 (108), 72.957(203), 
and $76.040(116)$. For the $2 \mathrm{H}-\mathrm{WS}_{2}$, all peaks are very sharp, indicating a well-crystallized and standard $2 \mathrm{H}$ structure. Its (002) is the strongest peak, followed by (103) as the second strongest. Compared with $2 \mathrm{H}-\mathrm{WS}_{2}$, the (002) peak of IF-WS 2 is left-shifted, indicating a lattice expansion of the (002) layers due to stains in the curved closed-cage layers [2]. The (103) and (105) peaks are broadened, attributing to the ultra-low dimensions. Whilst the (002) remains as the strongest peak, two peaks representing (100) and (101) have merged into one exhibiting the second highest intensity. The (006) and (104) peaks also merged, at around 44.1 degree. The (102) peak at around 35.9 degree was not detected in the IF-WS 2 profile. The small peaks appeared at 23-25 degree in the IF-WS pattern were assigned to $\mathrm{WO}_{x}$, which must exist as the residue core of some IF-WS 2 particles.

Figure S9. XRD profiles of commercial $2 \mathrm{H}-\mathrm{WS}_{2}$ and currently synthesised IF-WS2.

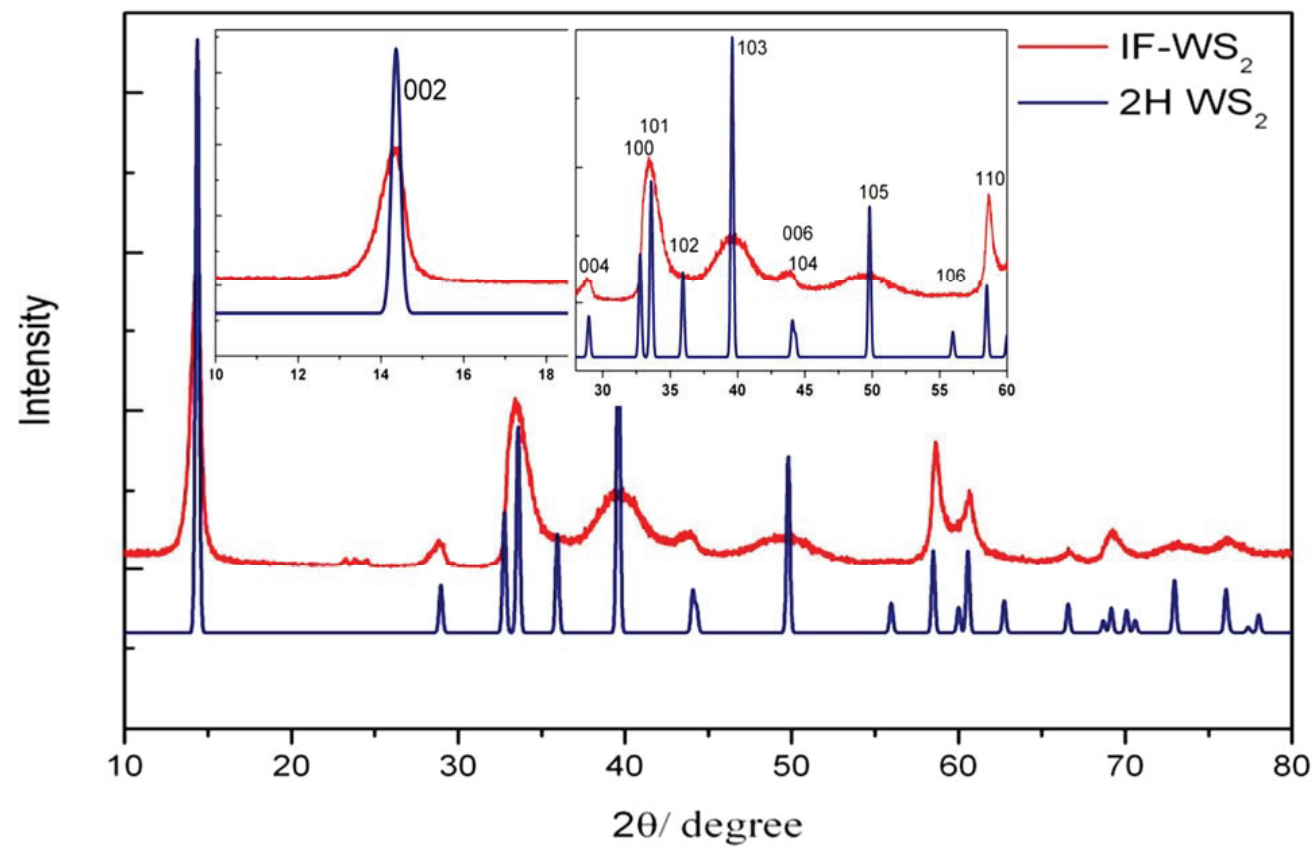

\subsection{Further Refinement and Modification}

2.3.1. IF-WS 2 Synthesis Using a Continuous Feeding System-Experiments Based on Both New Feeding System and Baffled Tubes are Named $\mathrm{FB}_{n}$

The XRD patterns of samples collected from Sample FB1 (Figure S10) demonstrate a completely conversion of $\mathrm{WO}_{x}$ into $\mathrm{WS}_{2}$. No $\mathrm{WO}_{x}$ peaks for the hot zone samples and extremely minor peaks of $\mathrm{WO}_{x}$ for the outlet zone samples. 
Figure S10. XRD profiles of samples collected from different reaction zones in experiment FB1.

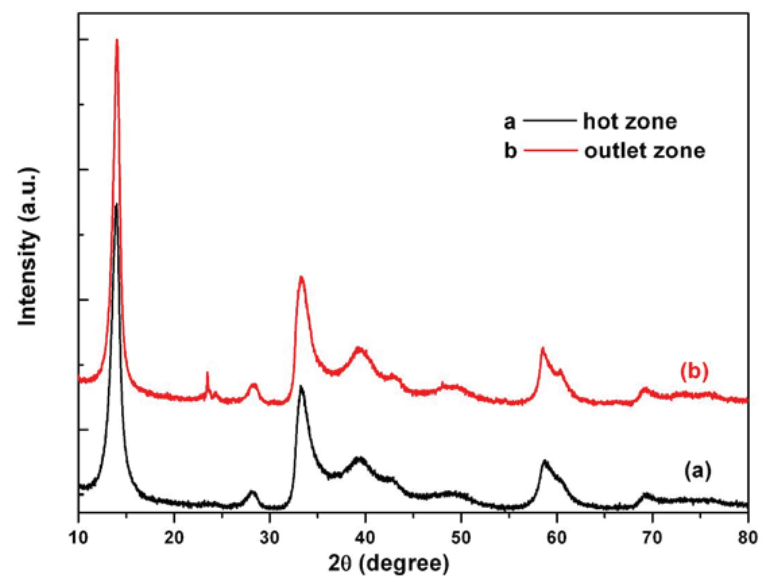

The SEM images in Figure S11 have revealed the morphology of particles collected from the hot zone in FB1. The particles were quite uniform, with sizes no more than $100 \mathrm{~nm}$. The very bright pieces at the right bottom corner of Figure S11b could be residue S, owing to the excessive amount of $\mathrm{H}_{2} \mathrm{~S}$ gas used.

Figure S11. SEM images for particles collected from hot zone of experiment FB1.
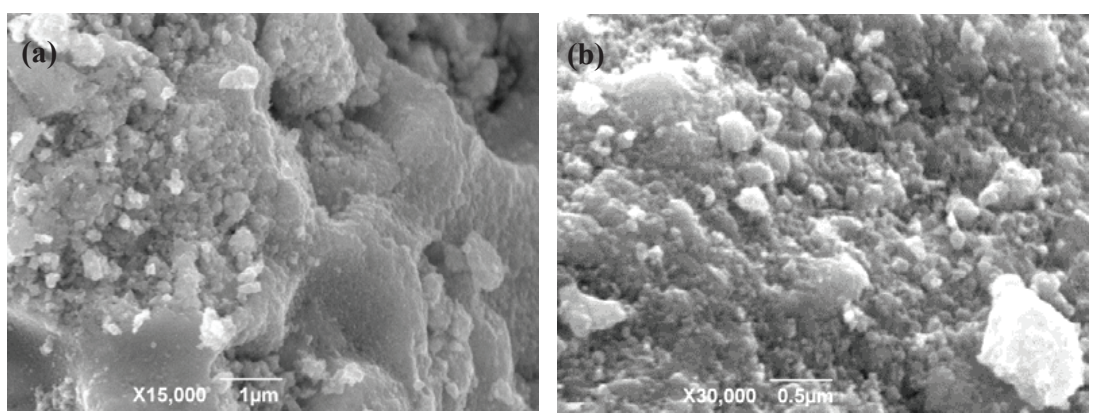

\section{References}

1. Feldman, Y.; Frey, G.L.; Homyonfer, M.; Lyakhovitskaya, V.; Margulis, L.; Cohen, H.; Hodes, G.; Hutchison, J.L.; Tenne, R. Bulk synthesis of inorganic fullerene-like $\mathrm{MS}_{2}(\mathrm{M}=\mathrm{Mo}, \mathrm{W})$ from the respective trioxides and the reaction mechanism. J. Am. Chem. Soc. 1996, 118, 5362-5367.

2. Feldman, Y.; Wasserman, E.; Srolovitz, D.J.; Tenne, R. High rate, gas phase growth of $\mathrm{MoS}_{2}$ nested inorganic fullerenes and nanotubes. Science 1995, 267, 222-225. 


\section{Acknowledgments}

The authors thank EPSRC (Engineering and Physical Sciences Research Council, UK) for financial support.

\section{Author Contributions}

Fang $\mathrm{Xu}$ designed, tested and modified the rotary furnace, conducted the experiments and drafted the manuscript; Nannan Wang and Hong Chang helped with some experiments and particle characterisations; Yongde Xia contributed to the data analyses; Yanqiu Zhu oversaw the entire project progress, and was the project leader.

\section{Conflicts of Interest}

The authors declare no conflict of interest.

\section{References}

1. Tenne, R.; Margulis, L.; Genut, M.; Hodes, G. Polyhedral and cylindrical structures of tungsten disulphide. Nature 1992, 360, 444-446.

2. Margulis, L.; Salitra, G.; Tenne, R.; Talianker, M. Nested fullerene-like structures. Nature 1993, 365, 113-114.

3. Feldman, Y.; Wasserman, E.; Srolovitz, D.J.; Tenne, R. High rate, gas phase growth of $\mathrm{MoS}_{2}$ nested inorganic fullerenes and nanotubes. Science 1995, 267, 222-225.

4. Chopra, N.G.; Luyken, R.J.; Cherrey, K.; Crespi, V.H.; Cohen, M.L.; Louie, S.G.; Zettl, A. Boron nitride nanotubes. Science 1995, 269, 966-967.

5. Rosenfeld, H.Y.; Grunbaum, E.; Tenne, R.; Sloan, J.; Hutchison, J.L. Cage structures and nanotubes of $\mathrm{NiCl}_{2}$. Nature 1998, 395, 336.

6. Vollath, D.; Szabo, D.V. Synthesis of nanocrystalline $\mathrm{MoS}_{2}$ and $\mathrm{WS}_{2}$ in a microwave plasma. Mater. Lett. 1998, 35, 236-244.

7. Mackie, E.B.; Galvan, D.H.; Adem, E.; Talapatra, S.; Yang, G.L.; Migone, A.D. Production of $\mathrm{WS}_{2}$ nanotubes by an activation method. Adv. Mater. 2000, 12, 495-498.

8. Remskar, M.; Skraba, Z.; Regula, M.; Ballif, C.; Sanjine's, R.; Levy, F. New crystal structures of $\mathrm{WS}_{2}$ : microtubes, ribbons, and ropes. Adv. Mater. 1998, 10, 246-249.

9. Li, Y.D.; Li, X.L.; He, R.R.; Zhu, J.; Deng, Z.X. Artificial lamellar mesostructures to WS 2 nanotubes. J. Am. Chem. Soc. 2002, 124, 1411-1416.

10. Li, X.L.; Ge, J.P.; Li, Y.D. Atmospheric pressure chemical vapor deposition: An alternative route to large-scale $\mathrm{MoS}_{2}$ and $\mathrm{WS}_{2}$ inorganic fullerene-like nanostructures and nanoflowers. Chem. A Eur. J. 2004, 10, 6163-6171.

11. Margolin, A.; Deepak, F.L.; Popovitz-Biro, R.; Bar-Sada, M.; Feldman, Y.; Tenne, R. Fullerene-like $\mathrm{WS}_{2}$ nanoparticles and nanotubes by the vapor-phase synthesis of $\mathrm{WCl}_{n}$ and $\mathrm{H}_{2} \mathrm{~S}$. Nanotechnology 2008, 19, doi:10.1088/0957-4484/19/9/095601. 
12. Seifert, G.; Terrones, H.; Terrones, M.; Jungnickel, G.; Frauenheim, T. Structure and electronic properties of $\mathrm{MoS}_{2}$ nanotubes. Phys. Rev. Lett. 2000, 85, 146-149.

13. Dominko, R.; Arcon, D.; Mrzel, A.; Zorko, A.; Cevc, P.; Venturini, P.; Gaberscek, M.; Remskar, M.; Mihailovic, D. Dichalcogenide nanotube electrodes for Li-Ion batteries. Adv. Mater. 2002, 14, 1531-1534.

14. Kaplan-Ashiri, I.; Tenne, R. Mechanical properties of WS2 nanotubes. J. Cluster Sci. 2007, 18, 549-563.

15. Rapoport, L.; Feldman, Y.; Homyonfer, M.; Cohen, H.; Sloan, J.; Hutchison, J.L.; Tenne, R. Inorganic fullerene-like material as additives to lubricants: Structure-function relationship. Wear 1999, 225-229, 975-982.

16. Kaplan-Ashiri, I.; Cohen, S.R.; Gartsman, K.; Ivanovskaya, V.; Heine, T.; Seifert, G.; Wiesel, I.; Wagner, H.D.; Tenne, R. On the mechanical behavior of $\mathrm{WS}_{2}$ nanotubes under axial tension and compression. Proc. Natl. Acad. Sci. 2006, 103, 523-528.

17. Rapoport, L.; Bilik, Y.; Feldman, Y.; Homyonfer, M.; Cohen, S.R.; Tenne, R. Hollow nanoparticles of $\mathrm{WS}_{2}$ as potential solid-state lubricants. Nature 1997, 387, 791-793.

18. Hou, X.H.; Shan, C.X.; Choy, K.L. Microstructures and tribological properties of PEEK-based nanocomposite coatings incorporating inorganic fullerene-like nanoparticles. Surf. Coat. Technol. 2008, 202, 2287-2291.

19. Brown, S.; Musfeldt, J.L.; Mihut, I.; Betts, J.B.; Migliori, A.; Zak, A.; Tenne, R. Bulk vs. nanoscale $\mathrm{WS}_{2}$ : Finite size effects and solid-state lubrication. Nano Lett. 2007, 7, 2365-2369.

20. Frey, G.L.; Elani, S.; Homyonfer, M.; Feldman, Y.; Tenne, R. Optical-absorption spectra of inorganic fullerene like $\mathrm{MS}_{2}(\mathrm{M}=\mathrm{Mo}, \mathrm{W})$. Phys. Rev. B 1998, 57, 6666-6671.

21. Zhu, Y.Q.; Sekine, T.; Li, Y.H.; Wang, W.X.; Fay, M.W.; Edwards, H.; Brown, P.D.; Fleischer, N.; Tenne, R. WS 2 and $\mathrm{MoS}_{2}$ inorganic fullerenes - super shock absorbers at very high pressures. Adv. Mater. 2005, 17, 1500-1503.

22. Zhu, Y.Q.; Sekine, T.; Li, Y.H.; Fay, M.W.; Zhao, Y.M.; Poa, C.H.P.; Wang, W.X.; Roe, M.J.; Brown, P.D.; Fleischer, N.; et al. Shock-absorbing and failure mechanisms of $\mathrm{WS}_{2}$ and $\mathrm{MoS}_{2}$ nanoparticles with fullerene-like structures under shock wave pressure. J. Am. Chem. Soc. 2005, 127, 16263-16272.

23. Zhu, Y.Q.; Sekine, T.; Brigatti, K.S.; Firth, S.; Tenne, R.; Rosentsveig, R.; Kroto, H.W.; Walton, D.R.M. Shock-wave resistance of $\mathrm{WS}_{2}$ nanotubes. J. Am. Chem. Soc. 2003, 125, 1329-1333.

24. Hogg, P.J. Composites in Armor. Science 2006, 314, 1100-1101.

25. Feldman, Y.; Zak, A.; Popovitz-Biro, R.; Tenne, R. New reactor for production of tungsten disulfide hollow onion-like (inorganic fullerene-like) nanoparticles. Solid State Sci. 2000, 2, 663-672.

26. Feldman, Y.; Frey, G.L.; Homyonfer, M.; Lyakhovitskaya, V.; Margulis, L.; Cohen, H.; Hodes, G.; Hutchison, J.L.; Tenne, R. Bulk synthesis of inorganic fullerene-like $\mathrm{MS}_{2}(\mathrm{M}=\mathrm{Mo}$, W) from the respective trioxides and the reaction mechanism. J. Am. Chem. Soc. 1996, 118, 5362-5367. 
27. Rothschild, A.; Sloan, J.; Tenne, R. Growth of $\mathrm{WS}_{2}$ nanotubes phases. J. Am. Chem. Soc. 2000, 122, 5169-5179.

28. Dai, J.J.; Cui, H.P.; Grace, J.R. Biomass feeding for thermochemical reactors. Prog. Energy Combust. Sci. 2012, 38, 716-736.

29. Wilson, D.H.; Dunnington, D.L. Plan ahead to avoid feeding problems. Chem. Eng. N. Y. 1991, 98, 72-81.

30. Hao, X.H.; Guo, L.J.; Mao, X.; Zhang, X.M.; Chen, X.J. Hydrogen production from glucose used as a model compound of biomass gasified in supercritical water. Int. J. Hydrogen Energy 2003, 28, 55-64.

31. Deepak, F.L.; Tenne, R. Gas-phase synthesis of inorganic fullerene-like, structures and inorganic nanotubes. Cent. Eur. J. Chem. 2008, 6, 373-389.

32. Rosentsveig, R.; Margolin, A.; Feldman, Y.; Popovitz-Biro, R.; Tenne, R. $\mathrm{WS}_{2}$ nanotube bundles and foils. Chem. Mater. 2002, 14, 471-474.

33. Margolin, A.; Rosentsveig, R.; Albu-Yaron, A.; Popovitz-Biro, R.; Tenne, R. Study of the growth mechanism of $\mathrm{WS}_{2}$ nanotubes produced by a fluidized bed reactor. J. Mater. Chem. 2004, 14, 617-624.

34. Alaei, M.; Rashidi, A.; Mahjoub, A. Two suitable methods for the preparation of inorganic fullerene-like (IF) $\mathrm{WS}_{2}$ nanoparticles. Iran. J. Chem. Chem. Eng. 2009, 28, 91-98.

35. Wiesel, I.; Arbel, H.; Albu-Yaron, A.; Popovitz-Biro, R.; Gordon, J.M.; Feuermann, D.; Tenne, R. Synthesis of $\mathrm{WS}_{2}$ and $\mathrm{MoS}_{2}$ fullerene-like nanoparticles from solid precursors. Nano Res. 2009, 2, 416-424.

36. Zhu, Y.Q.; Hsu, W.K.; Terrones, H.; Grobert, N.; Chang, B.H.; Terrones, M.; Wei, B.Q.; Kroto, H.W.; Walton, D.R.M.; Boothroyd, C.B.; et al. Morphology, structure and growth of $\mathrm{WS}_{2}$ nanotubes. J. Mater. Chem. 2000, 10, 2570-2577.

37. Li, Y.H.; Zhao, Y.M.; Ma, R.Z.; Zhu, Y.Q.; Fisher, N.; Jin, Y.Z.; Zhang, X.P. Novel route to $\mathrm{WO}_{x}$ nanorods and $\mathrm{WS}_{2}$ nanotubes from $\mathrm{WS}_{2}$ inorganic fullerenes. J. Phys. Chem. B 2006, 110, 18191-18195.

38. Zhu, Y.Q.; Hu, W.B.; Hsu, W.K.; Terrones, M.; Grobert, N.; Hare, J.P.; Kroto, H.W.; Walton, D.R.M.; Terrones, H. Tungsten oxide tree-like structures. Chem. Phys. Lett. 1999, 309, 327-334.

39. Zak, A.; Sallacan-Ecker, L.; Margolin, A.; Feldman, Y.; Popovitz-Biro, R.; Albu-Yaron, A.; Genut, M.; Tenne, R. Scaling up of the $\mathrm{WS}_{2}$ nanotubes synthesis. Fullerenes Nanotubes Carbon Nanostruct. 2011, 19, 18-26.

40. Zak, A.; Sallacan-Ecker, L.; Margolin, A.; Genut, M.; Tenne, R. Insight into the growth mechanism of $\mathrm{WS}_{2}$ nanotubes in the scaled-up fluidized-bed reactor. Nano 2009, 4, 91-98.

41. Finnie, G.J.; Kruyt, N.P.; Ye, M.; Zeilstra, C.; Kuipers, J.A.M. Longitudinal and transverse mixing in rotary kilns: A discrete element method approach. Chem. Eng. Sci. 2005, 60, 4083-4091. 


\title{
Gas-Phase and Microsolvated Glycine Interacting with Boron Nitride Nanotubes. A B3LYP-D2* Periodic Study
}

\author{
Albert Rimola and Mariona Sodupe
}

Abstract: The adsorption of glycine (Gly) both in gas-phase conditions and in a microsolvated state on a series of zig-zag $(n, 0)$ single-walled boron nitride nanotubes (BNNTs, $n=4,6,9$ and 15) has been studied by means of B3LYP-D2* periodic calculations. Gas-phase Gly is found to be chemisorbed on the $(4,0),(6,0)$ and $(9,0)$ BNNTs by means of a dative interaction between the $\mathrm{NH}_{2}$ group of Gly and a B atom of the BNNTs, whose computed adsorption energies are gradually decreased by increasing the tube radius. On the $(15,0)$ BNNT, Gly is found to be physisorbed with an adsorption driving force mainly dictated by $\pi$-stacking dispersion interactions. Gly adsorption in a microsolvated environment has been studied in the presence of seven water molecules by progressively microsolvating the dry Gly/BNNT interface. The most stable structures on the $(6,0)$, $(9,0)$ and $(15,0)$ BNNTs present the Gly/BNNT interface fully bridged by the water solvent molecules; i.e., no direct contact between Gly and the BNNTs takes place, whereas on the $(4,0)$ BNNT the most stable structure presents a unique direct interaction between the $\mathrm{COO}^{-}$Gly group and a $\mathrm{B}$ atom of the nanotube. Further energetic analyses indicate that the $(6,0),(9,0)$ and $(15,0)$ BNNTs exhibit a low water affinity, which favors the Gly/water interactions upon BNNT coadsorption. In contrast, the $(4,0)$ BNNT has been found to show a large water affinity, bringing the replacement of adsorbed water by a microsolvated glycine molecule as an unfavorable process.

Reprinted from Inorganics. Cite as: Rimola, A.; Sodupe, M. Gas-Phase and Microsolvated Glycine Interacting with Boron Nitride Nanotubes. A B3LYP-D2* Periodic Study. Inorganics 2014, 2, $334-350$.

\section{Introduction}

Bioconjugated nanostructured materials resulting from the coupling of biomolecules with inorganic nanomaterials including nanotubes, nanowires, nanoparticles and nanosheets have attracted much attention during the last years as they exhibit unique features derived from combining synergistically the properties of the interacting components. These exclusive physico-chemical properties render these materials as suitable substrates with potential applications in diverse biological- [1,2] and material-related [3] areas such as biocatalysis,[4,5], drug delivery [6-8], biosensing [9-13] and medical diagnostics [14,15]. The functionalities resulting from these biohybrid materials are largely mediated by the biomolecule/inorganic surface interactions, which in turn are dictated by the structure-specific binding properties of the two partners. Accordingly, precise knowledge on the interactions between the biomolecule and the inorganic components is of fundamental relevance.

Among the different nanostructured materials, boron nitride nanotubes (BNNTs) have been proposed to be suitable candidates to be combined with biomolecules [16]. BNNTs are isosteres and structurally similar to carbon nanotubes (CNTs), in which alternating B and N atoms substitute for C 
atoms. However, these two type of nanotubes exhibit different physico-chemical properties. Whereas CNTs exhibit metallic or semiconducting behavior, which moreover is strongly dependent on the tube diameter, helicity and concentric layers, BNNTs are electrical insulators with a band gap of $c a .5 .5 \mathrm{eV}$ regardless of the tube geometry features [17]. Moreover, at variance with the non-polar C-C bonds in CNTs, the B-N bonds of BNNTs exhibit a certain polar character, the degree of which depends on the curvature of the nanotube. That is, the increase of the tube curvature induces the transformation of the $\mathrm{sp}^{2}$ hybrid character of the $\mathrm{B}$ and $\mathrm{N}$ atoms in large diameter BNNTs into a $\mathrm{sp}^{3}$ one in small diameter BNNTs. As recently shown by us [18], this has important consequences for the nature of interaction between functional molecules and the BNNTs walls; i.e., polar molecules strongly chemisorb on small radius BNNTs, whereas interaction of non-polar molecules are energetically more favourable when physisorbed on large radius BNNTs. Furthermore, unlike CNTs, which present an inherent cytotoxicity [19], BNNTs have been found to be nontoxic [20] due to their high chemical and structural stability and high oxidation resistance, which alongside their uniformity and stability in dispersion in solution [21] make them suitable for biomedical applications.

Different experimental studies have focused on the interaction of peptides and proteins with BNNTs, showing a natural affinity between the two conjugates, which allows a direct immobilization of proteins on the BNNTs [22] as well as the isolation of individual BNNTs through a novel pathway based on peptide wrapping [23]. Moreover, biofunctionalized BNNTs via glycine interaction are good reactant substrates to obtain polysaccharide-coated BNNTs under mild conditions, in which the role of glycine is crucial during the interfacial process. The interactions of DNA and RNA with BNNTs have also been addressed and exploited to obtain nematic ordered ensembles of BNNT [24]. Other works have been devoted to assess the cytotoxicity of BNNTs when in contact with cells. Chen et al. [20] concluded that pristine BNNTs are inherently non-cytotoxic in view of the non-altered growth of human embryonic kidney (HEK) cells when cultured with BNNTs. Similar results were found by Ciofani and coworkers, in which coated-BNNTs presented a good cytocompatibility with human cells [25-28]. However, Goldberg and coworkers more recently found that BNNTs are actually cytotoxic for cells present in the lung alveoli and for HEK, in which the discrepancies with the other works were discussed and suggested to be due to the different morphology and size distribution of the BNNTs tested and the different assay techniques [29].

Theoretical works, mainly based on density functional theory (DFT) methods, have also studied the interaction of biomolecules with boron nitride nanostructures, most studies being limited to biomolecule building blocks, (amino acids and DNA and RNA nucleobases) due to the demanding computational cost of these calculations. Works on the gas-phase interaction of nucleobases using the local density approximation (LDA) and generalized gradient approximation (GGA) DFT levels of theory showed that this depends on the individual polarizations of the nucleobases [30-32]. The interaction of BNNTs with glycine (Gly) among other different amines has been studied in the gas-phase revealing an affinity of the BNNT with the $\mathrm{NH}_{2} \mathrm{Gly}$ group [33]. Study on the gas-phase interaction of the arginine (Arg), aspartic acid (Asp) and tryptophane (Trp) amino acids, with basic, acidic and aromatic side chain functionalities, respectively, at the LDA DFT level revealed that the binding is accompanied by charge transfer following the trend of Arg > Asp > Trp [34]. The binding of different biomolecules inside the cavity of BNNTs has also been studied at the LDA level [35]. 
The calculated weak interactions led the authors to suggest BNNTs to be suitable biological carriers due to the limited delivery kinetic barrier.

All these works focus on the intrinsic adsorption properties; i.e., they are limited to the gas-phase and, accordingly, solvation effects were not accounted for. Moreover, each work addresses the interaction of biomolecules with a particular BNNT. Since it has been shown that the tubular radius can modulate the adsorption properties of BNNTs [18], which is also applicable for biomolecules, the nature of interaction can significantly be different depending on the radius of the BNNT. Moreover, for the particular works addressing the interaction of amino acids, no conformational exploration to find out the most stable amino acid/BNNT adduct was performed (i.e., the initial amino acid conformation guesses were the most stable gas-phase structure), which is an important drawback due to the large conformational mobility of these molecules. In order to provide a more complete atomic-scale description of the interaction of amino acids with BNNTs, the present work reports a systematic periodic B3LYP-D2* study, using a hybrid functional and including dispersion corrections, on the interaction of Gly with different zig-zag $(n, 0)$ single-walled BNNTs $(n=4,6,9$ and 15) rendering nanotubes of different radius. Note that dispersive effects, not included in previous works, are expected to play a role in these systems. Moreover, with the aim to study in a more realistic way the interactions between biological systems and BNNTs, the very same Gly/BNNTs interaction study has been addressed considering a microsolvated environment modeled by the presence of seven water molecules. The effect of water has been analyzed from a structural and energetic point of view, with particular attention paid to the Gly/BNNT interface to determine whether the interaction is direct or bridged by the water molecules.

\section{Results and Discussion}

\subsection{Gas-Phase Interaction}

An exhaustive description relative to the modeling of the different BNNTs used in this work in their pristine state is available in a very recent work by us [18]. The BNNTs are automatically generated by the CRYSTAL code through the wrapping of a periodic boron nitride monolayer (hexagonal $\mathrm{P} 3 \mathrm{ml}$ layer symmetry group) into cylinders of different radius and fully exploiting the symmetry operators of the nanotubes [36-40]. In the interest of the present work, it is worth mentioning that the calculated electrostatic potential maps indicate a prominent positive/negative valued potential region for the $(4,0) \mathrm{BNNT}$, which become progressively less pronounced for BNNT with increasing radius, until obtaining a practically shallow electrostatic potential for the $(15,0)$ BNNT. For the interaction with Gly, the geometry optimizations were carried out as 1D polymers within the P1 space group, in which the unit cell parameters have been enlarged twice to avoid lateral interactions between molecules of adjacent unit cells.

The different optimized adducts for the adsorption of Gly on the BNNTs are shown in Figure 1, whereas the calculated adsorption energies alongside the pure electronic and dispersion contributions are shown in Table 1 . The adsorption energies ( $\left.\Delta E_{\text {ads }}\right)$ per unit cell of the probe molecules with the BNNTs are computed as: 


$$
\Delta E_{\mathrm{ads}}=E(\mathrm{Gly} / \mathrm{BNNT})-E(\mathrm{BNNT})-E_{\mathrm{m}}(\mathrm{Gly})
$$

where $E(\mathrm{Gly} / \mathrm{BNNT})$ is the energy of a fully relaxed unitary cell containing the BNNT in interaction with Gly, $E(\mathrm{BNNT})$ is the energy of a fully relaxed unitary cell of the BNNTs alone, and $E_{\mathrm{m}}(\mathrm{Gly})$ is the molecular energy of the free Gly.

Figure 1. (a) B3LYP-D2* optimized structures of the different calculated adducts for glycine interacting with the considered boron nitride nanotubes (BNNTs) in the gasphase; (b) B3LYP-D2* optimized structures for those complexes in which a spontaneous proton transfer occurs during the geometry optimization (see text). Distances in Å: bare values for the $(4,0)$ BNNT; values in parenthesis for the $(6,0)$ one; values in brackets for the $(9,0)$ one; and italic underlined values for the $(15,0)$ one.
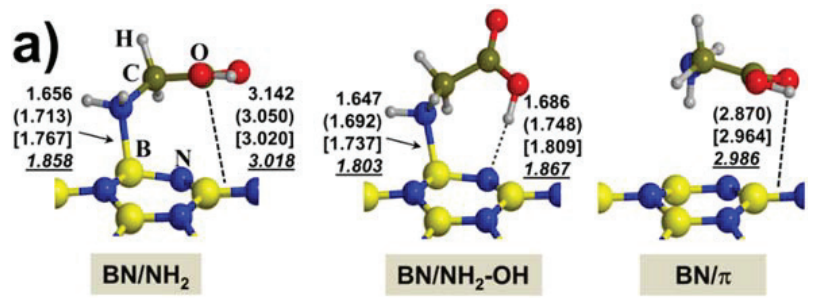

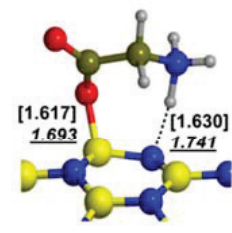

BN/zwitt

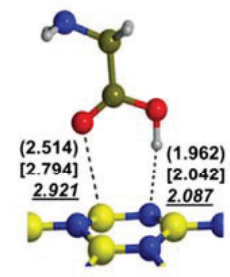

$\mathrm{BN} / \mathrm{COOH}-1$

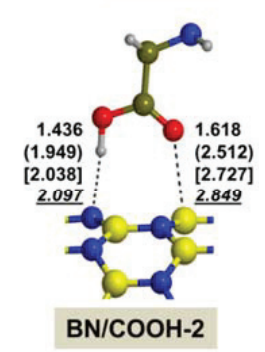

$\mathrm{BN} / \mathrm{COOH}-2$

b)
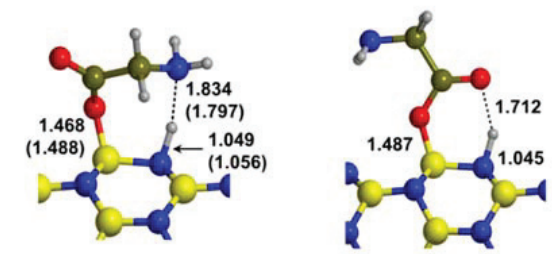

For all the BNNT systems, six initial structural guesses were considered (see Figure 1a): (i) pure interaction between the $\mathrm{NH}_{2}$ group of Gly and one nanotube $\mathrm{B}$ atom (hereafter referred to as $\mathrm{BN} / \mathrm{NH}_{2}$ ); (ii) $\mathrm{BN} / \mathrm{NH}_{2}$ interaction plus H-bonding between the Gly $\mathrm{OH}$ group and one nanotube $\mathrm{N}$ atoms (hereafter referred as $\mathrm{BN} / \mathrm{NH}_{2}-\mathrm{OH}$ ); (iii) and (iv) interaction between the Gly $\mathrm{CO}$ group and one nanotube $\mathrm{B}$ atom plus $\mathrm{H}$-bonding between the Gly $\mathrm{OH}$ group and one nanotube $\mathrm{N}$ atom, with the difference that the nanotube $\mathrm{B}$ and $\mathrm{N}$ atoms are chemically bonded or not (hereafter referred to as $\mathrm{BN} / \mathrm{COOH}-1$ and $\mathrm{BN} / \mathrm{COOH}-2$, respectively); (v) interaction of Gly in its zwitterionic form (hereafter referred to as $\mathrm{BN} / \mathrm{zwitt}$ ), in which the Gly $\mathrm{COO}^{-}$group interacts with one $\mathrm{B}$ atom and the 
Gly $\mathrm{NH}_{3}{ }^{+}$is $\mathrm{H}$-bonded to one nanotube $\mathrm{N}$ atom; and (vi) interaction between Gly and the BNNTs purely through the $\pi$ system of the Gly $\mathrm{COOH}$ group (hereafter referred to as $\mathrm{BN} / \pi$ ).

Data reported in Table 1 clearly indicate that the most stable adduct for the $(4,0)$ BNNT is the $\mathrm{BN} / \mathrm{NH}_{2}-\mathrm{OH}$ whereas for the $(6,0) \mathrm{BNNT}$ soth $\mathrm{BN} / \mathrm{NH}_{2}$ and $\mathrm{BN} / \mathrm{NH}_{2}-\mathrm{OH}$ are nearly degenerate. These complexes result from dative covalent interactions between the $\mathrm{Gly} \mathrm{NH}_{2}$ group and the $\mathrm{B}$ atoms, which act as Lewis acid sites. These findings are consistent with the data reported for the interaction of these BNNTs with probe molecules, in which the interaction of $\mathrm{NH}_{3}$ with the $(4,0)$ and $(6,0)$ BNNTs was found to be the strongest one among all tested molecules [18]. The fact that the $\mathrm{BN} / \mathrm{NH}_{2}-\mathrm{OH}$ adduct becomes the most stable one for the Gly/BNNT(4,0) system is consistent with the large polar character of this nanotube, the $\mathrm{N}$ atoms acting as $\mathrm{H}$-bonding acceptor groups. This is not in line with the most stable $\mathrm{BN} / \mathrm{NH}_{2}$ adduct found for the $(6,0) \mathrm{BNNT}$ and is due to the weaker $\mathrm{H}$-bonding acceptor character of the $\mathrm{N}$ nanotube atom when increasing the nanotube radius, which is reflected by an increase of the $\mathrm{H}$-bond distance in the $\mathrm{BN} / \mathrm{NH}_{2}-\mathrm{OH}$ adducts $(1.686$ and $1.748 \AA$ for the $(4,0)$ and $(6,0)$ BNNTs, see Figure 1a). In the same way, the B-N $\mathrm{N}_{\text {Gly }}$ bond lengths of the dative interactions in the $\mathrm{BN} / \mathrm{NH}_{2}$ and $\mathrm{BN} / \mathrm{NH}_{2}-\mathrm{OH}$ complexes also increase with increases in the nanotube radius due to the progressive decrease of the Lewis acceptor character of the $\mathrm{B}$ atom. Because of that, the calculated adsorption energy $\left(\Delta E_{\text {ads }}\right)$ is more negative and larger for the most stable $\mathrm{BN} / \mathrm{NH}_{2}-\mathrm{OH}$ adduct of Gly/BNNT(4,0) than the most stable BN/NH2 adduct of Gly/BNNT(6,0) (i.e., -33.2 and $-18.9 \mathrm{kcal}$ $\mathrm{mol}^{-1}$, respectively). The calculated energetic contributions; i.e., purely electronic and dispersion $\left(\Delta E_{\mathrm{el}}\right.$ and $\Delta E_{\mathrm{D}}$ values of Table 1 , respectively) indicate that Gly adsorption on the $(4,0)$ BNNT is largely dictated by the covalent dative interaction, whereas on the $(6,0)$ BNNT $\Delta E_{\text {el }}$ decreases in favor of dispersion. An analysis of the Mulliken charges (Q values of Table 2, only limited to the most stable Gly/BNNT complexes) confirms the formation of charge transfer complexes for both the Gly/BNNT $(4,0)$ and Gly/BNNT $(6,0)$ systems, the computed charge transfer values from Gly to the BNNTs being 0.30 e and $0.22 \mathrm{e}$, respectively.

The most stable Gly/BNNT(15,0) adduct has been found to be the $\mathrm{BN} / \pi$ one. This is in perfect agreement with the EPM results, which point out the $(15,0)$ BNNT as a practically non-polar nanomaterial. Data reported in Table 1 clearly indicate that the binding mechanism involved in this adduct is mainly based on dispersive forces (calculated $\Delta E_{\text {ads }}$ is practically equal to $\Delta E_{\mathrm{D}}$; i.e., -10.2 and $-11.7 \mathrm{kcal} \mathrm{mol}^{-1}$, respectively) dictated by $\pi$-stacking interactions between the $\pi$ systems of the $\mathrm{COOH}$ group and the B-N hexagon rings of the $(15,0)$ BNNT. Because of the presence of only non-covalent interactions, $\Delta E_{\text {ads }}$ is less negative compared to Gly interaction on $(4,0)$ and $(6,0)$ BNNTs. It is worth mentioning that the very same $\mathrm{BN} / \pi$ complexes have also been calculated for the Gly/BNNT $(4,0)$ and $(6,0)$ systems, meaning that, in the former case, the structure collapses onto the $\mathrm{BN} / \mathrm{NH}_{2}$ complex, whereas for the latter case the calculated $\Delta E_{\text {ads }}$ is found to be $9.0 \mathrm{kcal} \mathrm{mol}^{-1}$ above the most stable one, due to the lower propensity of this BNNT to establish $\pi$-stacking interactions. 
Table 1. Calculated adsorption energies $\left(\Delta E_{\text {ads }}\right)$, including the pure electronic energy contribution $\left(\Delta E_{\mathrm{el}}\right)$ and the contribution of dispersion $\left(\Delta E_{\mathrm{D}}\right)$. The relative electronic energies $\left(\Delta E_{\text {rel }}\right)$ for a given Gly/BNNT system are also included. Values in units of kcal $\mathrm{mol}^{-1}$.

\begin{tabular}{|c|c|c|c|c|c|}
\hline System & Adduct & $\Delta \boldsymbol{E}_{\mathrm{el}}$ & $\Delta \boldsymbol{E}_{\mathrm{D}}$ & $\Delta \boldsymbol{E}_{\mathrm{ads}}$ & $\Delta \boldsymbol{E}_{\text {rel }}$ \\
\hline \multirow[t]{6}{*}{ Gly/BNNT(4,0) } & $\mathrm{BN} / \mathrm{NH}_{2}$ & -20.8 & -11.2 & -31.9 & 1.3 \\
\hline & $\mathrm{BN} / \mathrm{NH}_{2}-\mathrm{OH}$ & -22.7 & -10.5 & -33.2 & 0.0 \\
\hline & $\mathrm{BN} / \pi$ & - & - & - & - \\
\hline & BN/zwitt & -20.7 & -10.4 & -31.2 & 2.0 \\
\hline & BN/COOH-1 & -24.6 & -7.1 & -31.7 & 2.2 \\
\hline & $\mathrm{BN} / \mathrm{COOH}-2$ & -9.8 & -7.8 & -17.6 & 15.6 \\
\hline \multirow[t]{6}{*}{ Gly/BNNT(6,0) } & $\mathrm{BN} / \mathrm{NH}_{2}$ & -6.3 & -12.6 & -18.9 & 0.0 \\
\hline & $\mathrm{BN} / \mathrm{NH}_{2}-\mathrm{OH}$ & -6.8 & -11.4 & -18.2 & 0.6 \\
\hline & $\mathrm{BN} / \pi$ & +0.5 & -10.4 & -9.9 & 9.0 \\
\hline & BN/zwitt & -1.8 & -11.7 & -13.5 & 5.4 \\
\hline & BN/COOH-1 & -3.3 & -6.3 & -9.6 & 8.0 \\
\hline & $\mathrm{BN} / \mathrm{COOH}-2$ & -3.3 & -6.2 & -9.5 & 8.1 \\
\hline \multirow[t]{6}{*}{ Gly/BNNT $(9,0)$} & $\mathrm{BN} / \mathrm{NH}_{2}$ & +1.5 & -13.5 & -12.0 & 0.0 \\
\hline & $\mathrm{BN} / \mathrm{NH}_{2}-\mathrm{OH}$ & +4.3 & -12.0 & -7.7 & 4.3 \\
\hline & $\mathrm{BN} / \pi$ & +1.0 & -11.1 & -10.1 & 1.9 \\
\hline & BN/zwitt & +14.0 & -12.5 & +1.5 & 13.5 \\
\hline & $\mathrm{BN} / \mathrm{COOH}-1$ & -2.6 & -6.0 & -8.6 & 3.4 \\
\hline & $\mathrm{BN} / \mathrm{COOH}-2$ & -2.2 & -6.1 & -8.3 & 3.7 \\
\hline \multirow[t]{6}{*}{ Gly/BNNT $(15,0)$} & $\mathrm{BN} / \mathrm{NH}_{2}$ & +7.2 & -14.3 & -7.1 & 3.1 \\
\hline & $\mathrm{BN} / \mathrm{NH}_{2}-\mathrm{OH}$ & +9.0 & -12.6 & -3.6 & 6.6 \\
\hline & $\mathrm{BN} / \pi$ & +1.5 & -11.7 & -10.2 & 0.0 \\
\hline & BN/zwitt & +20.7 & -13.1 & +7.6 & 17.8 \\
\hline & BN/COOH-1 & -1.4 & -6.1 & -7.5 & 2.7 \\
\hline & $\mathrm{BN} / \mathrm{COOH}-2$ & -1.4 & -6.2 & -7.6 & 2.6 \\
\hline
\end{tabular}

Table 2. Muliken charge (Q) of Gly adsorbed on the BNNTs in the most stable adducts and respective calculated direct band gaps $\left(E_{\mathrm{g}}\right)$.

\begin{tabular}{llll}
\hline System & Adduct & $\mathbf{Q}(\mathbf{e})$ & $\boldsymbol{E}_{\boldsymbol{g}}(\mathbf{e V})^{a}$ \\
\hline Gly/BNNT(4,0) & $\mathrm{BN} / \mathrm{NH}_{2}$ & 0.30 & 3.68 \\
& $\mathrm{BN} / \mathrm{NH}_{2}-\mathrm{OH}$ & 0.22 & 3.69 \\
\hline \multirow{2}{*}{ Gly/BNNT $(6,0)$} & $\mathrm{BN} / \mathrm{NH}_{2}$ & 0.23 & 4.46 \\
& $\mathrm{BN} / \mathrm{NH}_{2}-\mathrm{OH}$ & 0.16 & 4.45 \\
\hline Gly/BNNT$(9,0)$ & $\mathrm{BN} / \mathrm{NH}_{2}$ & 0.19 & 5.29 \\
& $\mathrm{BN} / \pi$ & -0.02 & 5.42 \\
\hline Gly/BNNT$(15,0)$ & $\mathrm{BN} / \pi$ & -0.02 & 6.06 \\
\hline${ }^{a}$ calculated direct band gaps for the pristine BNNTs: $3.67,4.42,5.42$ and $5.99 \mathrm{eV}$ for $(4,0),(6,0),(9,0)$ \\
and $(15,0)$, respectively.
\end{tabular}

The interaction of Gly with the $(9,0)$ BNNT is a frontier case between small radius (i.e., $(4,0)$ and $(6,0))$ and large radius (i.e., $(15,0))$ BNNTs. Although the $\mathrm{BN} / \mathrm{NH}_{2}$ adduct has been found to be the most stable one, the $\mathrm{BN} / \pi$ complex is the second most stable one lying $1.9 \mathrm{kcal} \mathrm{mol}^{-1}$ above. Calculated $\Delta E_{\text {ads }}$ values, however, can suffer from the basis set superposition error (BSSE). Indeed, 
upon correction, results indicate that these two complexes are nearly degenerate (BSSE-corrected $\Delta E_{\text {ads }}$ values being -9.4 and $-8.9 \mathrm{kcal} \mathrm{mol}^{-1}$ for the $\mathrm{BN} / \mathrm{NH}_{2}$ and $\mathrm{BN} / \pi$, respectively).

The interaction of Gly through the $\mathrm{COOH}$ group by means of a simultaneous $\mathrm{CO}-\mathrm{B}$ dative bond and a $\mathrm{OH}^{\cdots} \mathrm{N}_{\text {(BNNT) }} \mathrm{H}$-bond has also been considered (see $\mathrm{BN} / \mathrm{COOH}-1$ and $\mathrm{BN} / \mathrm{COOH}-2$ adducts). Although none of the calculated complexes are the most stable ones, important structural and energetic features deserve to be mentioned. For the BN/COOH-1 adduct, Gly adsorption on the $(4,0)$ BNNT results in a spontaneous proton transfer from the $\mathrm{Gly} \mathrm{COOH}$ group to the $\mathrm{N}$ nanotube atom, hence forming a COO-/BNNT- $\mathrm{H}^{+}$ion pair (see Figure 1b, structure of right). Such a proton transfer was already observed for the adsorption of $\mathrm{HCOOH}$ on the very same $(4,0) \mathrm{BNNT}$ and is attributed to the net charge transfer occurring from Gly to the BNNT, which induces an increase of the $\mathrm{COOH}$ acidity and the nanotube basicity up to the point of promoting the proton transfer to a nearby $\mathrm{N}$ atom of the nanotube. Moreover, for this adduct the CO-B distance is significantly shorter than for the other BN/COOH-1 adducts (1.487 $\AA$ versus 2.514-2.921 $\AA$, respectively), which results in a stronger interaction $\left(\Delta E_{\text {ads }}=-31.7 \mathrm{kcal} \mathrm{mol}^{-1}\right.$ and $\approx-9.6--7.5 \mathrm{kcal} \mathrm{mol}^{-1}$, respectively). For the BN/COOH-2 complex on the $(4,0) \mathrm{BNNT}$, no proton transfer has been found; although the $\mathrm{OH} \cdots \mathrm{N}$ (BNNT) $\mathrm{H}$-bond and the $\mathrm{CO}-\mathrm{B}$ dative bond are actually shorter than those present in the other BNNTs, in line with what has been described for the $\mathrm{BN} / \mathrm{COOH}-1$ cases. Interestingly, the difference between $\mathrm{BN} / \mathrm{COOH}-1$ and $\mathrm{BN} / \mathrm{COOH}-2$ is that in the former the $\mathrm{COOH}$ interaction occurs on $\mathrm{B}$ and $\mathrm{N}$ atoms that are chemically bonded to each other, whereas in the latter this is not the case. Accordingly, the fact that the spontaneous proton transfer only occurs in the former system seems to indicate that the charge transfer in enhanced by a cooperative effect between the $\mathrm{OH} \cdots \mathrm{N}_{(\mathrm{BNNT})}$ and the $\mathrm{CO}-\mathrm{B}$ interactions when the $\mathrm{B}$ and the $\mathrm{N}$ atoms are chemically bonded, which is in agreement with the larger and more negative calculated $\Delta E_{\text {ads }}$ values ( -31.7 and $-17.6 \mathrm{kcal} \mathrm{mol}^{-1}$ for $\mathrm{BN} / \mathrm{COOH}-1$ and $\mathrm{BN} / \mathrm{COOH}-2$, respectively).

Finally, it is worth mentioning that the interaction of Gly in its zwitterionic state has also been computed (BN/zwitt). On the $(4,0)$ and $(6,0)$ BNNTs, a spontaneous proton transfer from the $\mathrm{NH}_{3}{ }^{+}$ group to the N nanotube atom has been found, whereas for the $(9,0)$ and $(15,0)$ BNNTs the zwitterionic form is maintained. Consistently, calculated $\Delta E_{\text {ads }}$ values are negative for the two former adducts ( -31.2 and $-13.5 \mathrm{kcal} \mathrm{mol}^{-1}$, respectively), whereas for the two latter ones they have been found to be positive $\left(+1.5\right.$ and $+7.6 \mathrm{kcal} \mathrm{mol}^{-1}$, respectively) and, accordingly, are not stable complexes.

\subsection{Microsolvated Interaction}

Here, results on the interaction of Gly with the BNNTs in the presence of seven water molecules are reported. We have introduced seven water molecules since this is the minimum number of water molecules to have a relatively complete first-solvation shell of Gly upon adsorption; i.e., three water molecules interacting with the $\mathrm{NH}_{3}{ }^{+}$group, two water molecules interacting with the $\mathrm{COO}^{-}$group and two more water molecules to complete the solvation shell. For these cases, the unit cell parameters of the BNNTs have been enlarged thrice to avoid lateral interactions between water molecules of adjacent unit cells. It is worth mentioning that a statistical sampling of the hypersurface of these systems can be carried out adopting either the molecular dynamics or the Monte Carlo approaches [41]. However, these calculations are extremely expensive at the ab-initio level adopting realistic models for BNNTs like the $(9,0)$ and the $(15,0)$ ones, which contain 129 and 201 atoms, 
respectively. For the present work, we have followed a different approach consisting of a progressive microsolvation of the dry interface at the Gly/BNNT structures. This microsolvation procedure consists of adding water molecules at the dry Gly/BNNT interface in such a way that Gly progressively loses direct contact with the BNNTs up to a point in which the interaction is fully bridged by water. This procedure has already been performed by some of us in other works for the interaction of Gly with silica [42] and hydroxyapatite [43] surfaces. Since the most stable state of Gly in water is the zwitterionic one, we considered the BN/zwitt adducts as initial guesses for the progressive microsolvation. For the $(4,0),(6,0)$ and $(9,0)$ BNNTs, the resulting structures are shown in Figure 2a. In the BN/CONH adduct, Gly directly interacts with the BNNTs in a similar fashion as

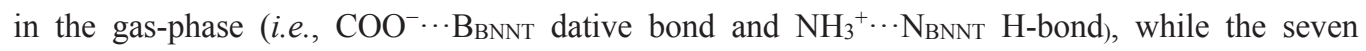
microsolvating water molecules are simple spectators interacting with available points of the $\mathrm{COO}^{-}$ and $\mathrm{NH}_{3}{ }^{+}$groups through $\mathrm{H}$-bonding. It is worth remarking that now for the $(4,0)$ and $(6,0)$ BNNTs no $\mathrm{H}$ transfer from Gly to the BNNT occurs (at variance with the gas-phase adsorption, vide supra) due to the screening effect of water. The $\mathrm{BN} / \mathrm{CO}-\mathrm{w} / \mathrm{NH}$ and $\mathrm{BN} / \mathrm{NH}-\mathrm{w} / \mathrm{CO}$ adducts result from moving one spectator water molecule from the outer shell to the inner shell so that the following water mediated interactions $\mathrm{NH}_{3}{ }^{+} \cdots \mathrm{H}_{2} \mathrm{O} \cdots \mathrm{N}_{\text {BNNT }}$ and $\mathrm{COO}^{-} \cdots \mathrm{H}_{2} \mathrm{O} \cdots$ B BNNT occur respectively. Finally, From these two adducts, a second water displacement to remove the remaining direct Gly/BNNT interaction gives the w/CONH adduct, in which water fully mediates the Gly/BNNT contact. It is worth mentioning that each of the seven $\mathrm{H}_{2} \mathrm{O}$ molecules can in principle be displaced from their positions to lead to a water mediated contact between Gly and BNNT. We choose the one exhibiting the weakest interaction energy with the other water molecules by computing the cost to remove one water molecule from the $\mathrm{BN} / \mathrm{CONH}$ adduct by a single point energy evaluation for each $\mathrm{H}_{2} \mathrm{O}$.

For the $(15,0) \mathrm{BNNT}$, all the optimization calculations collapsed to structures with no direct contact between Gly and BNNT, the most stable one being presented in Figure 2b. In this structure, water fully solvates the Gly molecule and, at variance with the other BNNT cases, no charge transfer between water and BNNT takes place, due to the highly apolar character of this nanotube.

The relative stabilities between the different calculated adducts for a given microsolvated complex are shown in Table 3 . As one can observe, for the $(6,0),(9,0)$ and $(15,0)$ BNNTs the most stable systems are the w/CONH adducts; i.e., those in which no direct Gly/BNNT contact occurs, whereas for the $(4,0)$ one, the $\mathrm{BN} / \mathrm{CO}-\mathrm{w} / \mathrm{NH}$ adduct (direct interaction only through the $\mathrm{COO}^{-}$) was found to be the most energetically stable one. It is worth mentioning, however, that the energy difference between the $\mathrm{BN}-\mathrm{CO}-\mathrm{w} / \mathrm{NH}$ and $\mathrm{w} / \mathrm{CONH}$ adducts for the $(4,0) \mathrm{BNNT}$ (the first and second most stable ones) is relatively small $\left(2.6 \mathrm{kcal} \mathrm{mol}^{-1}\right)$ and, accordingly, it might be inverted due to entropic effects associated with water rearrangement, as it is shown for peptide adsorption on hydrophobic and polar surfaces [44]. To further analyze this point, finite temperature molecular dynamics simulations would be desirable. 
Figure 2. B3LYP-D2* optimized structures of the different calculated complexes for glycine interacting with the $(4,0),(6,0)$ and $(9,0)$ BNNTs (a) and with the $(15,0)$ BNNT (b) in the presence of seven water molecules. Distances in $\AA$ : bare values for the $(4,0)$ BNNT; values in parenthesis for the $(6,0)$ one; values in brackets for the $(9,0)$ one; and italic underscored values for the $(15,0)$ one. For this latter case, the distance is that between the $\mathrm{C}$ atom and the plane defined by the closest $\mathrm{B}-\mathrm{N}$ hexagon ring.

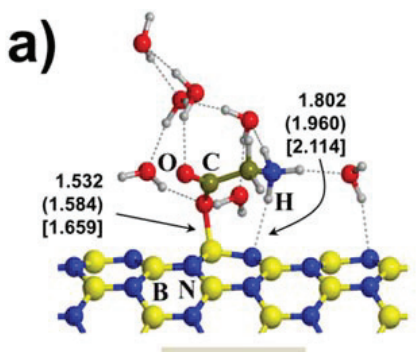

BN/CONH

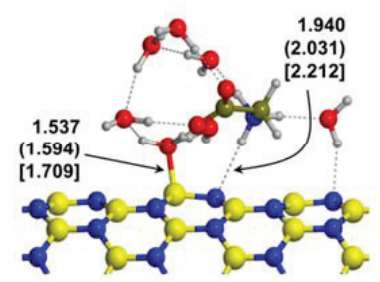

BN/NH-w/CO

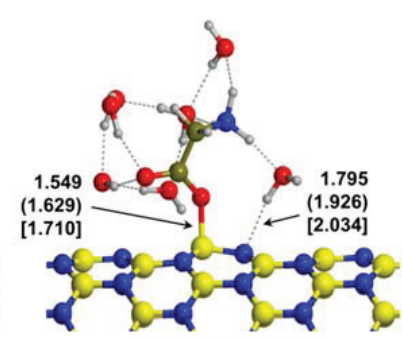

BN/CO-w/NH

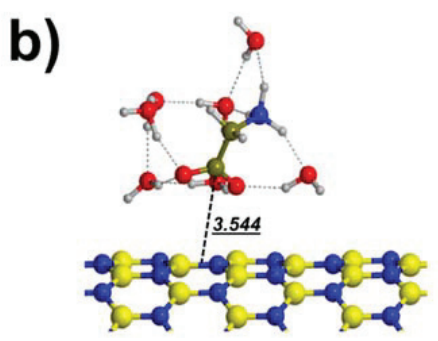

Besides these results, three different processes have moreover been considered to study the stability of the structures shown in Figure 2. The first one involves Gly solvated by $7 \mathrm{H}_{2} \mathrm{O}$ molecules being adsorbed on the clean walls of the BNNTs, whose reaction energy was computed as (reported by $\Delta E_{\mathrm{R} 1}$ of Table 2)

$$
\mathrm{Gly} / 7 \mathrm{w}+\mathrm{BNNT} \rightarrow \mathrm{Gly} / 7 \mathrm{w} / \mathrm{BNNT}
$$

where Gly/7w is glycine solvated by the seven $\mathrm{H}_{2} \mathrm{O}$ molecules and Gly/7w/BNNT represents the microsolvated complexes. The $\Delta E_{\mathrm{R} 1}$ column shows that for all BNNTs the process is exoenergetic, meaning that the structural rearrangement of the seven $\mathrm{H}_{2} \mathrm{O}$ molecules around Gly is compensated by the interaction with the BNNTs. Remarkably, limited to the most favorable adducts per BNNT, 
$\Delta E_{\mathrm{R} 1}$ values are less negative with increases in the nanotube radius, consistent with the less polar behavior of the BNNTs.

Table 3. Reaction energies $\left(\Delta E_{\mathrm{R} 1}, \Delta E_{\mathrm{R} 2}\right.$ and $\left.\Delta E_{\mathrm{R} 3}\right)$ and relative energies $\left(\Delta E_{\mathrm{rel}}\right)$ of the formation of the Gly/7w/BNNT complexes. Values in units of $\mathrm{kcal} \mathrm{mol}^{-1}$.

\begin{tabular}{llllll}
\hline System & Adduct & $\Delta \boldsymbol{E}_{\text {rel }}$ & $\Delta \boldsymbol{E}_{\mathrm{R} 1}$ & $\Delta \boldsymbol{E}_{\mathrm{R} 2}$ & $\Delta \boldsymbol{E}_{\mathrm{R} 3}$ \\
\hline Gly/7w/BNNT(4,0) & BN/CONH & 5.4 & -28.9 & -11.5 & 11.9 \\
& BN/CO-w/NH & 0.0 & -34.3 & -16.9 & 6.5 \\
& BN/NH-w/CO & 4.3 & -30.0 & -12.6 & 10.8 \\
& w/CONH & 2.6 & -31.7 & -14.3 & 9.1 \\
\hline Gly/7w/BNNT(6,0) & BN/CONH & 5.3 & -13.2 & -20.5 & 2.9 \\
& BN/CO-w/NH & 2.9 & -15.7 & -23.0 & 0.4 \\
& BN/NH-w/CO & 4.2 & -14.4 & -21.7 & 1.7 \\
& w/CONH & 0.0 & -18.6 & -25.9 & -2.5 \\
\hline Gly/7w/BNNT(9,0) & BN/CONH & 12.4 & -5.1 & -20.7 & 2.7 \\
& BN/CO-w/NH & 7.7 & -9.8 & -25.4 & -2.0 \\
& BN/NH-w/CO & 10.5 & -7.0 & -22.6 & 0.8 \\
& w/CONH & 0.0 & -17.5 & -33.1 & -9.7 \\
\hline Gly/7w/BNNT(15,0) & BN/CONH & - & - & - & - \\
& BN/CO-w/NH & - & - & - & - \\
& BN/NH-w/CO & - & - & - & - \\
& w/CONH & 0.0 & -12.8 & -31.4 & -8.1 \\
\hline
\end{tabular}

The second process envisages gas phase Gly adsorbed on the already microsolvated BNNTs by the seven water molecules, whereas the third one envisages the solvated Gly being adsorbed on the seven water hydrated BNNTs giving rise to the shown adducts with expulsion of seven water molecules (here considered as a H-bonded cluster), whose reaction energies were computed as (reported by $\Delta E_{\mathrm{R} 2}$ and $\Delta E_{\mathrm{R} 3}$ of Table 2)

$$
\begin{gathered}
\text { Gly }+7 \mathrm{w} / \mathrm{BNNT} \rightarrow \mathrm{Gly} / 7 \mathrm{w} / \mathrm{BNNT} \\
\mathrm{Gly} / 7 \mathrm{w}+7 \mathrm{w} / \mathrm{BNNT} \rightarrow \mathrm{Gly} / 7 \mathrm{w} / \mathrm{BNNT}+7 \mathrm{w}
\end{gathered}
$$

where $7 \mathrm{w} / \mathrm{BNNT}$ is the BNNT solvated by the seven $\mathrm{H}_{2} \mathrm{O}$ molecules and $7 \mathrm{w}$ the H-bonded cluster made up by seven water molecules. These last two processes require the BNNTs in the presence of seven water molecules, whose optimized structures are given in Figure 3. The initial guess of these systems were the corresponding w/CONH adducts (no direct interaction between Gly and the BNNTs and accordingly the interaction between water molecules and BNNTs is maximum) in which the Gly molecule was removed. Consistent with the polar character of the BNNTs, several interactions between the water molecules and the $(4,0)$ BNNT via covalent dative and $\mathrm{H}$-bond interactions take place, whereas by increasing the BNNT radius these interactions are progressively missed, up to the point in which for the $(15,0)$ no apparent interaction is observed. 
Figure 3. B3LYP-D2* optimized structures of the different calculated complexes for the considered BNNTs in the presence of seven water molecules. Distances in $\AA$ : bare values for the $(4,0)$ BNNT; values in parenthesis for the $(6,0)$ one; values in brackets for the $(9,0)$ one; and italic underscored values for the $(15,0)$ one. For this latter case, the distance is that between the closest water $\mathrm{O}$ to the plane defined by the $\mathrm{B}-\mathrm{N}$ hexagon ring.

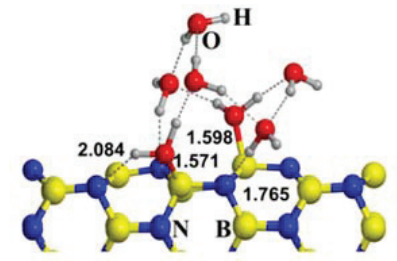

7 w/BNNT(4,0)

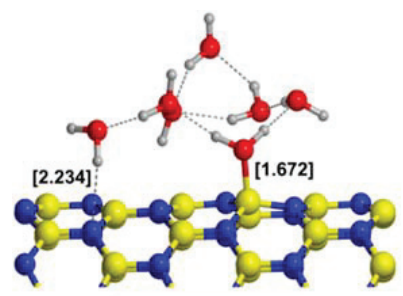

7 w/BNNT(9,0)

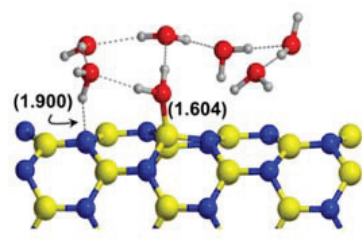

7 w/BNNT(6,0)

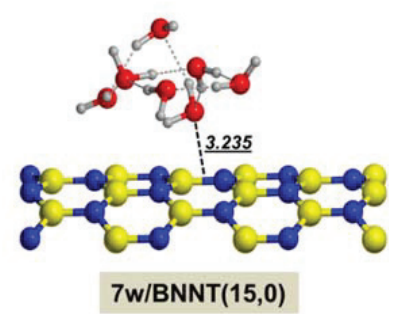

The energetics of R2 is a tradeoff between the water affinity of the BNNTs and Gly (R2 is in essence the capture of the adsorbed water by Gly). The trend provided by the calculated $\Delta E_{\mathrm{R} 2}$ values indicates that R2 is more favorable when increasing the radius. That is, since the $(15,0)$ BNNT does not exhibit water affinity, the calculated $\Delta E_{\mathrm{R} 2}$ values are large and negative due to the strong interaction of water with Gly. In contrast, both the $(4,0)$ BNNT and Gly exhibit large water affinity and accordingly the calculated $\Delta E_{\mathrm{R} 2}$ values are the less negative ones of the series. R3 is probably the most physically sound process as it involves the replacement of adsorbed water solvent by an already solvated Gly. Calculated $\Delta E_{\mathrm{R} 3}$ values indicate that such a water replacement is energetically favorable on the $(15,0)$ and $(9,0)$ BNNTs probably due to the low water affinity of these BNNTs. On the $(6,0)$ the process is still favorable by some amount but less than on the other two nanotubes $\left(\Delta E_{\mathrm{R} 3}=-2.5 \mathrm{kcal} \mathrm{mol}^{-1}\right)$, whereas on the $(4,0)$ calculated $\Delta E_{\mathrm{R} 1}$ was found to be positive, indicating that the overall interactions between Gly, water solvent and the nanotube are not as stable as the interaction between this nanotube and water.

\section{Computational Details}

All calculations were carried out using the periodic ab-initio code CRYSTAL09 [45]. All the SCF calculations and geometry optimizations were performed using the B3LYP-D* density functional method, which includes an empirical a posteriori correction term proposed by Grimme [46] to account for dispersion forces (missed in the pure B3LYP $[47,48]$ method), but whose initial parameterization (D) was modified for extended systems (D*) [49], to provide accurate results for the calculations of 
cohesive energies of molecular crystals and of adsorption processes within a periodic treatment [49-51]. The adopted Gaussian functions consisted of an all electron triple- $\zeta$ 6-311G* standard basis set for the $\mathrm{B}$ and $\mathrm{N}$ atoms of the BNNTs and a TZP basis set from Ahlrichs and coworkers [52] for the atoms of Gly. This basis set combination has been proved to exhibit small basis set superposition error interaction energies $[18,50]$. The shrinking factor of the reciprocal space net defining the mesh of $\mathrm{k}$ points in the irreducible Brillouin zone was set to 5, which requires diagonalizing the Hamiltonian matrix in $3 \mathrm{k}$ points [53]. The accuracy of both Coulomb and exchange series was set to values of overlap integrals of $10^{-7}$ and $10^{-16}$, respectively, which ensure a very good numerical accuracy. A pruned $(75,974)$ grid has been used for the Gauss-Legendre and Lebedev quadrature schemes in the evaluation of functionals. The condition to achieve SCF convergence between two subsequent cycles was set to $10^{-7} \mathrm{Eh}$. Full relaxations of both lattice parameters and internal atomic coordinates by means of analytical energy gradients [54-56] have been carried out. The geometry optimization was performed by means of a quasi-Newton algorithm in which the quadratic step (BFGS Hessian updating scheme) is combined with a linear one (parabolic fit) [57].

\section{Conclusions}

Periodic quantum mechanical calculations have been used to simulate the adsorption of glycine (Gly) on different zig-zag $(n, 0)$ single-walled boron-nitride nanotubes (BNNTs, $n=4,6,9$ and 15) both in the gas-phase and in a microsolvated state (i.e., modeled by the presence of seven explicit water molecules) with the aim of determining the adsorption properties and the effect exerted by water as a function of surface curvature. These calculations are based on the B3LYP-D2* method, which includes the B3LYP hybrid functional with a revised version of the empirical a posteriori correction term $(\mathrm{D} 2 *)$ to account for dispersion interactions.

Gas-phase results clearly indicate that the most stable interaction between Gly and the $(4,0),(6,0)$ and $(9,0)$ BNNTs takes place through a covalent dative interaction between the $\mathrm{NH}_{2}$ group of Gly and the $\mathrm{B}$ atom of the BNNTs, which produce charge transfers from Gly to the BNNTs. In contrast, the interaction between Gly and the $(15,0)$ BNNT is mainly governed by non-covalent dispersive forces based on a $\pi$-stacking between the $\pi$ systems of the Gly COOH group and the B-N hexagon rings of the nanotube. Remarkably, the energy difference between these two adducts decreases when increasing the BNNT radius, in line with the polar/apolar character of the considered nanotubes.

The adsorption of Gly on the BNNTs in the presence of seven water molecules has been studied adopting a progressive microsolvation procedure, in which water solvent molecules are added at the dry Gly/BNNT interfaces, hence progressively removing the direct interaction between Gly and the BNNTs. The obtained results indicate that for the $(6,0),(9,0)$ and $(15,0)$ BNNTs, the most stable microsolvated systems were found to exhibit no direct contact between the two partners; that is, the Gly/BNNT interfaces are fully bridged by the water solvent molecules. In contrast, for the $(4,0)$ one of the most stable systems shows direct contact between Gly and the BNNT through an interaction between the Gly $\mathrm{COO}^{-}$group and a nanotube B atom, although entropic effects (not accounted for in this work) might favor water mediated interface. Further energetic results point out that the larger the BNNT radius, the less water affinity. Accordingly, for larger radius BNNTs, the interaction between water and Gly was found to be predominant, in detriment to their interaction with the BNNT. 
However, it is found that the $(4,0)$ BNNT exhibits a large water affinity, which is reflected by the fact that the replacement of seven adsorbed water molecules by a microsolvated Gly has been found to be an unfavorable process.

The results presented here provide evidence that the adsorption properties of the BNNTs as well as their water affinity can significantly be modulated by controlling the tube diameter, as they are expected to exhibit different physico-chemical features, which may be of interest for the design of bioconjugated systems based on boron-nitride nanostructures and their potential bionanotechnological applications.

\section{Acknowledgments}

Albert Rimola is indebted to Ministry of Economy (MINECO) of the Spanish Government for a Juan de la Cierva contract. Financial support from MINECO (projects CTQ2013-40347-ERC and CTQ2011-24847/BQU) and from Department of Economy of the Catalan Government (project 2009SGR-638), and the use of the Catalonia Supercomputer Centre (CESCA) are gratefully acknowledged. Mariona Sodupe also acknowledges support through the 2011 ICREA Academia award.

\section{Author Contributions}

Simulations were carried out by Albert Rimola. Both Albert Rimola and Mariona Sodupe contributed to the discussion and writing of the paper.

\section{Conflicts of Interest}

The authors declare no conflict of interest.

\section{References}

1. Kujawa, P.; Winnik, F.M. Innovation in Nanomedicine through Materials Nanoarchitectonics. Langmuir 2013, 29, 7354-7361.

2. Wong, S.S.; Joselevich, E.; Woolley, A.T.; Cheung, C.L.; Lieber, C.M. Covalently functionalized nanotubes as nanometre- sized probes in chemistry and biology. Nature 1998, 394, 52-55.

3. Aono, M.; Bando, Y.; Ariga, K. Nanoarchitectonics: Pioneering a New Paradigm for Nanotechnology in Materials Development. Adv. Mater. 2012, 24, 150-151.

4. Hong, R.; Fischer, N.O.; Verma, A.; Goodman, C.M.; Emrick, T.; Rotello, V.M. Control of Protein Structure and Function through Surface Recognition by Tailored Nanoparticle Scaffolds. J. Am. Chem. Soc. 2004, 126, 739-743.

5. You, C.-C.; Agasti, S.S.; De, M.; Knapp, M.J.; Rotello, V.M. Modulation of the Catalytic Behavior of $\alpha$-Chymotrypsin at Monolayer-Protected Nanoparticle Surfaces. J. Am. Chem. Soc. 2006, 128, 14612-14618.

6. Kang, Y.; Liu, Y.-C.; Wang, Q.; Shen, J.-W.; Wu, T.; Guan, W.-J. On the spontaneous encapsulation of proteins in carbon nanotubes. Biomaterials 2009, 30, 2807-2815. 
7. Gao, H.; Kong, Y. Simulation of DNA-nanotube interactions. Annu. Rev. Mater. Res. 2004, 34, 123-150.

8. Chen, X.; Kis, A.; Zettl, A.; Bertozzi, C.R. A cell nanoinjector based on carbon nanotubes. Proc. Natl. Acad. Sci. USA 2007, 104, 8218-8222.

9. Chen, R.J.; Bangsaruntip, S.; Drouvalakis, K.A.; Wong Shi Kam, N.; Shim, M.; Li, Y.; Kim, W.; Utz, P.J.; Dai, H. Noncovalent functionalization of carbon nanotubes for highly specific electronic biosensors. Proc. Natl. Acad. Sci. USA 2003, 100, 4984-4989.

10. Chen, R.J.; Zhang, Y.; Wang, D.; Dai, H. Noncovalent Sidewall Functionalization of Single-Walled Carbon Nanotubes for Protein Immobilization. J. Am. Chem. Soc. 2001, 123, 3838-3839.

11. Cui, Y.; Wei, Q.; Park, H.; Lieber, C.M. Nanowire Nanosensors for Highly Sensitive and Selective Detection of Biological and Chemical Species. Science 2001, 293, 1289-1292.

12. Staii, C.; Johnson, A.T.; Chen, M.; Gelperin, A. DNA-Decorated Carbon Nanotubes for Chemical Sensing. Nano Lett. 2005, 5, 1774-1778.

13. Heller, D.A.; Jeng, E.S.; Yeung, T.-K.; Martinez, B.M.; Moll, A.E.; Gastala, J.B.; Strano, M.S. Optical Detection of DNA Conformational Polymorphism on Single-Walled Carbon Nanotubes. Science 2006, 311, 508-511.

14. Pantarotto, D.; Partidos, C.D.; Graff, R.; Hoebeke, J.; Briand, J.-P.; Prato, M.; Bianco, A. Synthesis, Structural Characterization, and Immunological Properties of Carbon Nanotubes Functionalized with Peptides. J. Am. Chem. Soc. 2003, 125, 6160-6164.

15. Tu, X.; Manohar, S.; Jagota, A.; Zheng, M. DNA sequence motifs for structure-specific recognition and separation of carbon nanotubes. Nature 2009, 460, 250-253.

16. Golberg, D.; Bando, Y.; Huang, Y.; Terao, T.; Mitome, M.; Tang, C.; Zhi, C. Boron Nitride Nanotubes and Nanosheets. ACS Nano 2010, 4, 2979-2993.

17. Golberg, D.; Bando, Y.; Tang, C.C.; Zhi, C.Y. Boron Nitride Nanotubes. Adv. Mater. 2007, 19, 2413-2432.

18. Rimola, A.; Sodupe, M. Physisorption vs. chemisorption of probe molecules on boron nitride nanomaterials: The effect of surface curvature. Phys. Chem. Chem. Phys. 2013, 15, 13190-13198.

19. Magrez, A.; Kasas, S.; Salicio, V.; Pasquier, N.; Seo, J.W.; Celio, M.; Catsicas, S.; Schwaller, B.; Forró, L. Cellular Toxicity of Carbon-Based Nanomaterials. Nano Lett. 2006, 6, 1121-1125.

20. Chen, X.; Wu, P.; Rousseas, M.; Okawa, D.; Gartner, Z.; Zettl, A.; Bertozzi, C.R. Boron Nitride Nanotubes Are Noncytotoxic and Can Be Functionalized for Interaction with Proteins and Cells. J. Am. Chem. Soc. 2009, 131, 890-891.

21. Yu, J.; Chen, Y.; Cheng, B.M. Dispersion of boron nitride nanotubes in aqueous solution with the help of ionic surfactants. Solid State Commun. 2009, 149, 763-766.

22. Zhi, C.; Bando, Y.; Tang, C.; Golberg, D. Immobilization of Proteins on Boron Nitride Nanotubes. J. Am. Chem. Soc. 2005, 127, 17144-17145.

23. Gao, Z.; Zhi, C.; Bando, Y.; Golberg, D.; Serizawa, T. Isolation of Individual Boron Nitride Nanotubes via Peptide Wrapping. J. Am. Chem. Soc. 2010, 132, 4976-4977.

24. Zhi, C.; Bando, Y.; Wang, W.; Tang, C.; Kuwahara, H.; Golberg, D. DNA-Mediated Assembly of Boron Nitride Nanotubes. Chem. Asian J. 2007, 2, 1581-1585. 
25. Ciofani, G.; Raffa, V.; Menciassi, A.; Dario, P. Preparation of Boron Nitride Nanotubes Aqueous Dispersions for Biological Applications. J. Nanosci. Nanotechnol. 2008, 8, 6223-6231.

26. Ciofani, G.; Raffa, V.; Menciassi, A.; Cuschieri, A. Cytocompatibility, interactions, and uptake of polyethyleneimine-coated boron nitride nanotubes by living cells: Confirmation of their potential for biomedical applications. Biotechnol. Bioeng. 2008, 101, 850-858.

27. Ciofani, G.; Danti, S.; D’Alessandro, D.; Moscato, S.; Menciassi, A. Assessing cytotoxicity of boron nitride nanotubes: Interference with the MTT assay. Biochem. Biophys. Res. Commun. 2010, 394, 405-411.

28. Ciofani, G.; Danti, S.; D’Alessandro, D.; Ricotti, L.; Moscato, S.; Bertoni, G.; Falqui, A.; Berrettini, S.; Petrini, M.; Mattoli, V.; et al. Enhancement of Neurite Outgrowth in Neuronal-Like Cells following Boron Nitride Nanotube-Mediated Stimulation. ACS Nano 2010, 4, 6267-6277.

29. Horváth, L.; Magrez, A.; Golberg, D.; Zhi, C.; Bando, Y.; Smajda, R.; Horváth, E.; Forró, L.; Schwaller, B. In Vitro Investigation of the Cellular Toxicity of Boron Nitride Nanotubes. ACS Nano 2011, 5, 3800-3810.

30. Akdim, B.; Kim, S.N.; Naik, R.R.; Maruyama, B.; Pender, M.J.; Pachter, R. Understanding effects of molecular adsorption at a single-wall boron nitride nanotube interface from density functional theory calculations. Nanotechnology 2009, 20, 355705.

31. Saikat, M.; Gowtham, S.; Ralph, H.S.; Ravindra, P.; Shashi, P.K. Theoretical study of physisorption of nucleobases on boron nitride nanotubes: A new class of hybrid nano-biomaterials. Nanotechnology 2010, 21, 165703.

32. Lin, Q.; Zou, X.; Zhou, G.; Liu, R.; Wu, J.; Li, J.; Duan, W. Adsorption of DNA/RNA nucleobases on hexagonal boron nitride sheet: An ab initio study. Phys. Chem. Chem. Phys. 2011, 13, 12225-12230.

33. Wu, X.; An, W.; Zeng, X.C. Chemical Functionalization of Boron-Nitride Nanotubes with $\mathrm{NH}_{3}$ and Amino Functional Groups. J. Am. Chem. Soc. 2006, 128, 12001-12006.

34. Mukhopadhyay, S.; Scheicher, R.H.; Pandey, R.; Karna, S.P. Sensitivity of Boron Nitride Nanotubes toward Biomolecules of Different Polarities. J. Phys. Chem. Lett. 2011, 2, 2442-2447.

35. Yang, C.-K. Exploring the interaction between the boron nitride nanotube and biological molecules. Comput. Phys. Commun. 2011, 182, 39-42.

36. Noel, Y.; D’Arco, P.; Demichelis, R.; Zicovich-Wilson, C.M.; Dovesi, R. On the Use of Symmetry in the Ab Initio Quantum Mechanical Simulation of Nanotubes and Related Materials. J. Comput. Chem. 2010, 31, 855-862.

37. Demichelis, R.; Noel, Y.; D’Arco, P.; Rerat, M.; Zicovich-Wilson, C.M.; Dovesi, R. Properties of Carbon Nanotubes: An ab Initio Study Using Large Gaussian Basis Sets and Various DFT Functionals. J. Phys. Chem. C 2011, 115, 8876-8885.

38. D’Arco, P.; Noel, Y.; Demichelis, R.; Dovesi, R. Single-layered chrysotile nanotubes: A quantum mechanical ab initio simulation. J. Chem. Phys. 2009, 131, doi:10.1063/1.3251791.

39. Demichelis, R.; Noel, Y.; D’Arco, P.; Maschio, L.; Orlando, R.; Dovesi, R. Structure and energetics of imogolite: A quantum mechanical ab initio study with B3LYP hybrid functional. J. Mater. Chem. 2010, 20, 10417-10425. 
40. Ferrari, A.M.; Szieberth, D.; Zicovich-Wilson, C.M.; Demichelis, R. Anatase(001) 3 ML Nanotubes, The First $\mathrm{TiO}_{2}$ Nanotube With Negative Strain Energies: A DFT Prediction. J. Phys. Chem. Lett. 2010, 1, 2854-2857.

41. Marx, D.; Hutter, J. Ab Initio Molcular Dynamics: Theory and Implementation. In Modern Methods and Algorithms of Quantum Chemistry; Grotendorst, J., Ed.; Forschungszentrum Jülich: Jülich, Germany, 2000.

42. Rimola, A.; Civalleri, B.; Ugliengo, P. Neutral vs. Zwitterionic Glycine Forms at the Water/Silica Interface: Structure, Energies, and Vibrational Features from B3LYP Periodic Simulations. Langmuir 2008, 24, 14027-14034.

43. Rimola, A.; Corno, M.; Zicovich-Wilson, C.M.; Ugliengo, P. Ab initio modeling of protein/biomaterial interactions: Competitive adsorption between glycine and water onto hydroxyapatite surfaces. Phys. Chem. Chem. Phys. 2009, 11, 9005-9007.

44. Schwierz, N.; Horinek, D.; Liese, S.; Pirzer, T.; Balzer, B.N.; Hugel, T.; Netz, R.R. On the Relationship between Peptide Adsorption Resistance and Surface Contact Angle: A Combined Experimental and Simulation Single-Molecule Study. J. Am. Chem. Soc. 2012, 134, 19628-19638.

45. Dovesi, R.; Saunders, V.R.; Roetti, C.; Orlando, R.; Zicovich-Wilson, C.M.; Pascale, F.; Civalleri, B.; Doll, K.; Harrison, N.M.; Bush, I.J.; et al. CRYSTAL09 User's Manual; University of Torino: Torino, Italy, 2009; p. 307.

46. Grimme, S. Semiempirical GGA-type density functional constructed with a long-range dispersion correction. J. Comput. Chem. 2006, 27, 1787-1799.

47. Becke, A.D. Density-functional thermochemistry. III. The role of exact exchange. J. Chem. Phys. 1993, 98, 5648-5652.

48. Lee, C.; Yang, W.; Parr, R.G. Development of the Colle-Salvetti correlation-energy formula into a functional of the electron density. Phys. Rev. B 1988, 37, 785-789.

49. Civalleri, B.; Zicovich-Wilson, C.M.; Valenzano, L.; Ugliengo, P. B3LYP augmented with an empirical dispersion term (B3LYP-D*) as applied to molecular crystals. CrystEngComm 2008, 10, 405-410.

50. Rimola, A.; Civalleri, B.; Ugliengo, P. Physisorption of aromatic organic contaminants at the surface of hydrophobic/hydrophilic silica geosorbents: A B3LYP-D modeling study. Phys. Chem. Chem. Phys. 2010, 12, 6357-6366.

51. Civalleri, B.; Maschio, L.; Ugliengo, P.; Zicovich-Wilson, C.M. Role of dispersive interactions in the $\mathrm{CO}$ adsorption on $\mathrm{MgO}$ (001): Periodic B3LYP calculations augmented with an empirical dispersion term. Phys. Chem. Chem. Phys. 2010, 12, 6382-6386.

52. Schafer, A.; Horn, H.; Ahlrichs, R. Fully optimized contracted Gaussian basis sets for atoms Li to Kr. J. Chem. Phys. 1992, 97, 2571-2577.

53. Monkhorst, H.J.; Pack, J.D. Special points for Brillouin-zone integrations. Phys. Rev. B 1976, 13, 5188-5192.

54. Doll, K.; Saunders, V.R.; Harrison, N.M. Analytical Hartree-Fock gradients for periodic systems. Int. J. Quantum Chem. 2001, 82, 1-13. 
55. Doll, K. Implementation of analytical Hartree-Fock gradients for periodic systems. Comput. Phys. Commun. 2001, 137, 74-88.

56. Doll, K.; Dovesi, R.; Orlando, R. Analytical Hartree-Fock gradients with respect to the cell parameter for systems periodic in three dimensions. Theor. Chem. Acc. 2004, 112, 394-402.

57. Civalleri, B.; D'Arco, P.; Orlando, R.; Saunders, V.R.; Dovesi, R. Hartree-Fock geometry optimisation of periodic systems with the CRYSTAL code. Chem. Phys. Lett. 2001, 348, 131-138. 


\title{
The Role of Lead (Pb) in the High Temperature Formation of $\mathrm{MoS}_{2}$ Nanotubes
}

\author{
Olga Brontvein, Reshef Tenne and Andrey Enyashin
}

Abstract: Recent studies have clearly indicated the favorable effect of lead as a growth promoter for $M X_{2}(M=\mathrm{Mo}, \mathrm{W} ; X=\mathrm{S}, \mathrm{Se})$ nanotubes using $M X_{2}$ powder as a precursor material. The experimental work indicated that the lead atoms are not stable in the molybdenum oxide lattice ion high concentration. The initial lead concentration in the oxide nanowhiskers ( $\mathrm{Pb}$ :Mo ratio $=0.28)$ is reduced by one order of magnitude after one year in the drawer. The initial $\mathrm{Pb}$ concentration in the $\mathrm{MoS}_{2}$ nanotubes lattice (produced by solar ablation) is appreciably smaller ( $\mathrm{Pb}$ :Mo ratio for the primary samples is 0.12 ) and is further reduced with time and annealing at $810{ }^{\circ} \mathrm{C}$, without consuming the nanotubes. In order to elucidate the composition of these nanotubes in greater detail; the Pb-"modified" $M X_{2}$ compounds were studied by means of DFT calculations and additional experimental work. The calculations indicate that $\mathrm{Pb}$ doping as well as $\mathrm{Pb}$ intercalation of $\mathrm{MoS}_{2}$ lead to the destabilization of the system; and therefore a high $\mathrm{Pb}$ content within the $\mathrm{MoS}_{2}$ lattice cannot be expected in the final products. Furthermore; substitutional doping ( $\mathrm{PbM}$ ) leads to p-type semiconducting character; while intercalation of $\mathrm{MoS}_{2}$ by $\mathrm{Pb}$ atoms $\left(\mathrm{Pb}_{y} / \mathrm{MoS}_{2}\right)$ should cause n-type semiconducting behavior. This study not only sheds light on the role of added lead to the growth of the nanotubes and their role as electron donors; but furthermore could pave the way to a large scale synthesis of the $\mathrm{MoS}_{2}$ nanotubes.

Reprinted from Inorganics. Cite as: Brontvein, O.; Tenne, R.; Enyashin, A. The Role of Lead (Pb) in the High Temperature Formation of $\mathrm{MoS}_{2}$ Nanotubes. Inorganics 2014, 2, 363-375.

\section{Introduction}

Inorganic nanotubes (INT) have become a prominent research subject in recent years. Their unique mechanical, optical and electrical properties [1-8] prompted extensive investigation. A number of synthetic routes have been developed, for example, chemical vapor transport using bromine for INT-MoS 2 [9,10], bismuth catalyzed vapor-liquid-solid method for $\mathrm{SnS}_{2}$ nanotubes [11], misfit compounds superstructures of $\mathrm{SnS}-\mathrm{SnS}_{2}$ and $\mathrm{NbS}_{-} \mathrm{PbS}_{2}$ nanotubes [12,13] and sulfidization of tungsten suboxide nanowhiskers leading to the formation of INT-WS 2 [14].

Recent studies have shown a new strategy for successful synthesis of $M X_{2}(M=\mathrm{Mo}, \mathrm{W} ; X=\mathrm{S}, \mathrm{Se})$ nanotubes using $\mathrm{Pb}$ as a growth promoter [15,16]. Figure 1 presents a SEM image of $\mathrm{MoS}_{2}$ nanotubes (Figure 1a) and high resolution TEM image of single $\mathrm{MoS}_{2}$ nanotube (Figure 1b) formed by irradiating $\mathrm{MoS}_{2}$ powder by a focused $(\times 15,000)$ sunlight for $10 \mathrm{~min}[15]$. The inset in Figure $1 \mathrm{~b}$ shows the distance profile of nanotube layers. The interlayer spacing of $0.634 \mathrm{~nm}$ is somewhat larger than that of bulk $2 \mathrm{H}-\mathrm{MoS}_{2}(0.62 \mathrm{~nm})$. This $2 \%$ expansion of the lattice spacing in the nanotubes is well documented [17] and is attributed to strain relaxation. Figure 1c shows the EDS spectrum of single $\mathrm{MoS}_{2}$ nanotube after the irradiation, the table in the inset in Figure 1c shows the atomic $\%$ of the elements. In both cases the formation mechanism can be described as a Pb-promoted $M X_{2}$ conversion 
into $\mathrm{Mo}(\mathrm{Pb}) \mathrm{O}_{3-x}$ nanowhiskers at high temperature $\left(>2500^{\circ} \mathrm{C}\right)$. Once formed, the nanowhiskers react back with the $\mathrm{X}$-vapor which leads to the formation of $M X_{2}$ nanotubes. Numerous attempts to synthesize $\mathrm{MoS}_{2}$ nanotubes in a conventional oven (up to $1000^{\circ} \mathrm{C}$ ) or induction furnace (up to $1600{ }^{\circ} \mathrm{C}$ ), with the same precursor materials, or generation of $\mathrm{Mo}(\mathrm{Pb}) \mathrm{O}_{3-x}$ nanowhiskers and their subsequent sulfidization did not succeed. It appears, therefore, that the high temperatures $\left(>2500{ }^{\circ} \mathrm{C}\right)$ and the presence of lead are critical for the conversion of $\mathrm{MoS}_{2}$ powder into $\mathrm{Mo}(\mathrm{Pb}) \mathrm{O}_{3-x}$ nanowhiskers which serve as template for the synthesis of $M X_{2}$ nanotubes according to this process. In addition, the use of $\mathrm{Pb}$ as a growth promoter is environmentally unfavorable. Therefore, stabilization of molybdenum suboxide phases could be done using different metals, for example V, Ta or W [18-20].

Figure 1. $\mathrm{MoS}_{2}$ nanotubes formed using solar ablation system (a) SEM image of $\mathrm{MoS}_{2}$ nanotubes; (b) high resolution TEM image of a single $\mathrm{MoS}_{2}$ nanotube (adapted after [15]). The inset shows the profile of the interlayer distances of the nanotube wall. The interlayer spacing is somewhat larger than that of bulk $2 \mathrm{H}-\mathrm{MoS}_{2}(0.62 \mathrm{~nm})$; (c) EDS spectrum of a single $\mathrm{MoS}_{2}$ nanotube; the inset table shows atomic \% of the nanotube's elements.

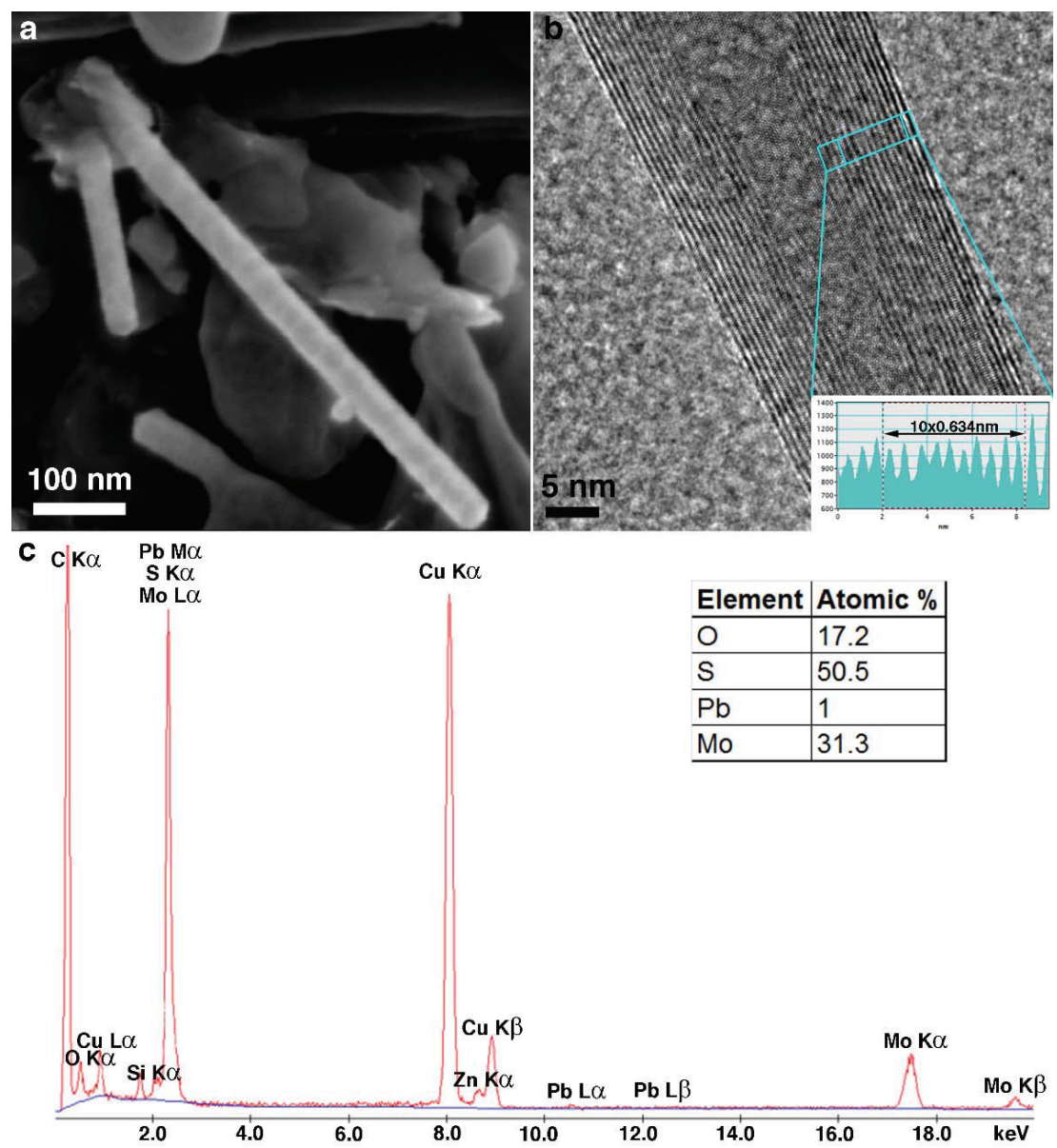


The main goal of the current work is to understand the role of lead $(\mathrm{Pb})$ as a growth promoter of INT-MoS2; evaluate its solubility limits in the two lattices (oxide and sulfide) and their electronic state in the INT-MoS 2 lattice. In particular, the stability of this heavy metal in the oxide precursor and the formed $\mathrm{MoS}_{2}$ nanotubes was investigated through both theory and experiment. Due to the fast kinetics of the process, the samples (oxide nanowhiskers and nanotubes) formed in such extreme conditions contain a certain amount of $\mathrm{Pb}$. However, the content of $\mathrm{Pb}$ is found to be time-dependent and can considerably decrease upon thermal annealing. DFT calculations give preliminary information about the coordination and the electronic state of $\mathrm{Pb}$ atoms within the $\mathrm{MoS}_{2}$ lattice. They verify a low affinity of the $\mathrm{Pb}$ atoms as dopants or intercalants in the sulfide matrices and indicate that the experimentally observed high amount of lead $(\mathrm{Pb})$ is too far from the thermodynamic equilibrium conditions.

\section{Results and Discussion}

\subsection{Annealing}

To distinguish between the different samples of oxide nanowhiskers and the molybdenum disulfide nanotubes studied in this work a labeling system was used (see Table 1). For this work, two different precursors were investigated: $\mathrm{MoO}_{3-x}$ (2) nanowhiskers and $\mathrm{MoS}_{2}$ (2) nanotubes, which were left one year in the drawer after solar ablation synthesis. The detailed description of their synthesis can be seen in the experimental part. Due to the small quantities of the $\mathrm{MoS}_{2}$ nanotubes and $\mathrm{MoO}_{3-x}$ nanowhiskers that are obtained in the synthesis $(\sim 1 \%-2 \%$ yield $)$ only single particle EDS measurement can be suitable for their chemical analysis.

Table 1. The samples labeling that were used during the experimental work.

\begin{tabular}{cccccc}
\hline Precursor & $\begin{array}{c}\text { After the Solar } \\
\text { Ablation Synthesis }\end{array}$ & $\begin{array}{c}\text { After a Year in } \\
\text { Ambient Conditions }\end{array}$ & $\begin{array}{c}\mathbf{3 0} \text { min } \\
\text { Annealing in } \mathbf{H}_{2} \mathbf{S}\end{array}$ & $\begin{array}{c}\mathbf{1} \text { h Annealing } \\
\text { in } \mathbf{H}_{2} \mathbf{S}\end{array}$ & $\begin{array}{c}\mathbf{2} \text { h Annealing } \\
\text { in } \mathbf{H}_{2} \mathbf{S}\end{array}$ \\
\hline $\mathrm{MoO}_{3-x}$ & $\mathrm{MoO}_{3-x}(1)$ & $\mathrm{MoO}_{3-x}(2)$ & $\mathrm{MoS}_{2}(3 \mathrm{a})$ & $\mathrm{MoS}_{2}(3 \mathrm{~b})$ & $\mathrm{MoS}_{2}(3 \mathrm{c})$ \\
$\mathrm{MoS}_{2}$ & $\mathrm{MoS}_{2}(1)$ & $\mathrm{MoS}_{2}(2)$ & $\mathrm{MoS}_{2}(4 \mathrm{a})$ & $\mathrm{MoS}_{2}(4 \mathrm{~b})$ & $\mathrm{MoS}_{2}(4 \mathrm{c})$ \\
\hline
\end{tabular}

The experimental work which has recently been carried-out indicated that the $\mathrm{Pb}$ atoms are not stable in high concentrations in the $\mathrm{MoO}_{3-x}$ or $\mathrm{MoS}_{2}$ lattice. According to the EDS measurements the initial lead concentration in the molybdenum suboxide nanowhiskers $\mathrm{MoO}_{3-x}$ (1) was reduced by one order of magnitude after one year in the drawer ( $\mathrm{Pb}$ :Mo ratio reduced from $\sim 0.28$ to 0.03 in $\mathrm{MoO}_{3-x}(2)$ ) (Figure 2). The same analysis showed that in the case of $\mathrm{MoS}_{2}$ (1) nanotubes the $\mathrm{Pb}$ concentration decreased from $\sim 0.12$ to 0.03 in $\mathrm{MoS}_{2}$; (2). Subsequent annealing of both types of nanostructures, i.e., $\mathrm{MoO}_{3-x}$ (2) nanowhiskers and $\mathrm{MoS}_{2}$ (2) nanotubes, leads to additional reduction of the $\mathrm{Pb}$ concentration. The $\mathrm{Pb}$ :Mo ratio before and after annealing calculated from EDS measurements can be seen in Figure 2. It should be emphasized that the accuracy of the EDS is greatly compromised at the lower concentration limit of $\mathrm{Pb}$.

The outdiffusion of the lead from the molybdenum oxide nanowhiskers $\mathrm{MoO}_{3-x}$ (2) did influence their high-temperature stability and their conversion into $\mathrm{MoS}_{2}$ (3) nanotubes upon annealing in $\mathrm{H}_{2} \mathrm{~S}$ atmosphere at $810^{\circ} \mathrm{C}$. In all cases, $\mathrm{MoS}_{2}(3,4)$ nanotubes were observed after the sulfidization and annealing of the lead-depleted $\mathrm{MoO}_{3-x}$ (2) nanowhiskers and $\mathrm{MoS}_{2}$ (2) nanotubes. TEM images of 
the precursors and products can be seen in Figure 3. Detailed description of the annealing conditions can be seen in the experimental section.

Figure 2. $\mathrm{Pb}: \mathrm{Mo}$ ratio of the precursors and products according to the EDS measurements, (a) $\mathrm{MoO}_{3-x}$ (2) nanowhiskers as a precursor material; (b) $\mathrm{MoS}_{2}$ (2) nanotubes as a precursor material.
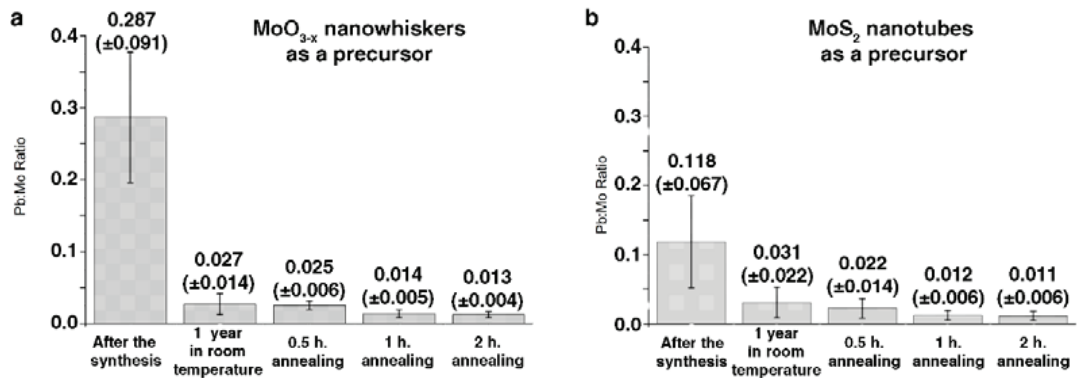

Due to the overlap between the $\mathrm{Mo}(\mathrm{L})$ and $\mathrm{Pb}(\mathrm{M})$ peaks $(2.3 \mathrm{KeV})$ in the EDS spectra, the calculated atomic concentration of $\mathrm{Pb}$ is based on the $\mathrm{Pb}(\mathrm{L})(10.5 \mathrm{KeV})$ and $\mathrm{Mo}(\mathrm{K})(17.4 \mathrm{KeV})$ peaks, which provide lower accuracy. Therefore, the detection of $\mathrm{Pb}$ atoms after a year in the drawer is limited to the detected range ( $>0.2$ at $\%$ ). On the other hand, it can be seen from the EDS spectra of the samples before and after annealing that in the case of molybdenum suboxide nanowhiskers $\mathrm{MoO}_{3-x}$ (2) as a precursor material, the $\mathrm{Pb}$ peak disappears after two hours annealing in $\mathrm{H}_{2} \mathrm{~S}$ atmosphere, while the same peak disappears after 30 min annealing at $810{ }^{\circ} \mathrm{C}$ in the case of $\mathrm{MoS}_{2}$ (2) nanotubes (Figure 4).

Figure 3. TEM images, (a) $\mathrm{MoO}_{3-x}$ (1) nanowhisker obtained after the synthesis (solar ablation for $30 \mathrm{~s}$ ); (b) $\mathrm{MoO}_{3-x}$ (2) nanowhisker a year after the synthesis; (c-e) $\mathrm{MoS}_{2}$ (3) nanotubes after $30 \mathrm{~min}, 1$ and $2 \mathrm{~h}$ of $\mathrm{H}_{2} \mathrm{~S}$ annealing, respectively, with $\mathrm{MoO}_{3-x}$ nanowhiskers as a precursor; (f) $\mathrm{MoS}_{2}$ (1) nanotubes after the synthesis (solar ablation for $10 \mathrm{~min}$ ); (g) $\mathrm{MoS}_{2}$ (2) nanotube a year after the synthesis, (h-j) $\mathrm{MoS}_{2}$ (4) nanotubes after $30 \mathrm{~min}, 1$ and $2 \mathrm{~h}\left(\mathrm{H}_{2} \mathrm{~S}\right)$ annealing, respectively, with $\mathrm{MoS}_{2}(2)$ nanotubes as a precursor.
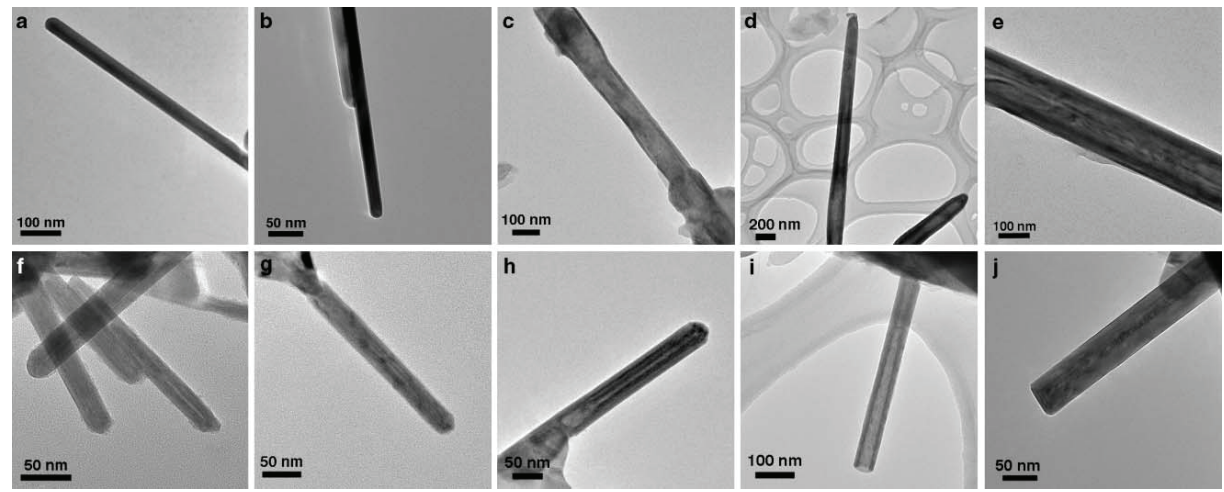
Figure 4. EDS spectra of the samples before and after annealing at $810^{\circ} \mathrm{C}$, (a) $\mathrm{MoO}_{3-x}$ (2) nanowhiskers as a precursor; (b) $\mathrm{MoS}_{2}$ (2) nanotubes as a precursor.

a $\mathrm{MoO}_{3-\mathrm{x}}$ nanowhiskers as a precursor

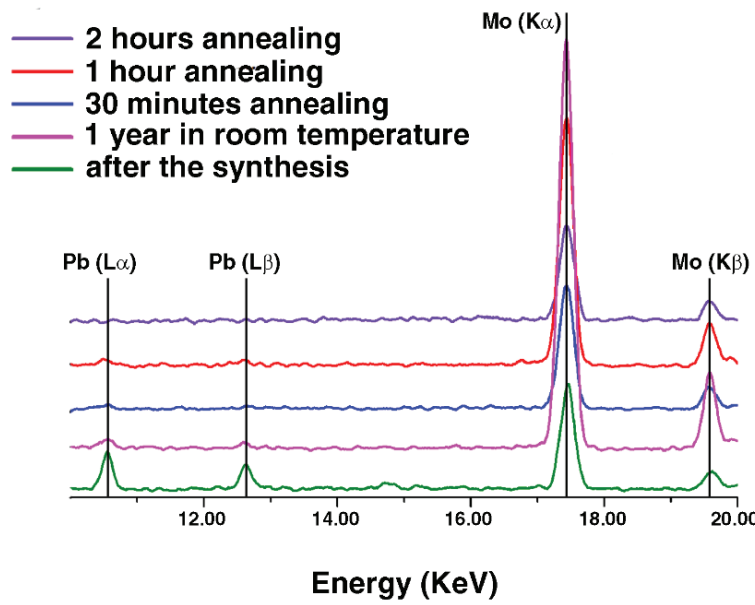

b $\mathrm{MoS}_{2}$ nanotubes as a precursor

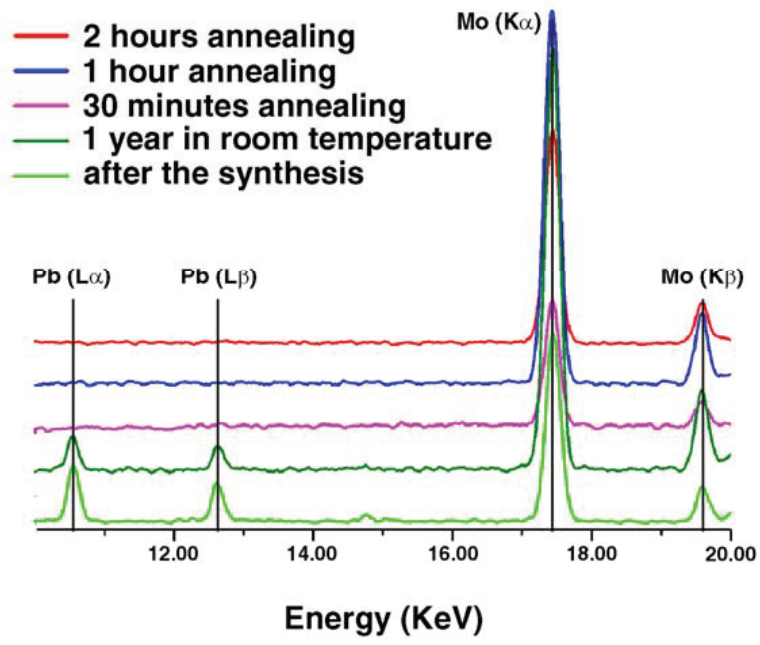

\subsection{DFT Calculations of $\mathrm{Mo}_{1-x} \mathrm{~Pb} b_{x} \mathrm{~S}_{2}$ Solid Solutions and $\mathrm{Pb} / \mathrm{MoS} \mathrm{S}_{2}$ Intercalates}

The experimental work has indicated a time-dependent content of $\mathrm{Pb}$ in the samples of $\mathrm{MoS}_{2}$ nanotubes after $\mathrm{Pb}$-promoted synthesis, which suggests that the $\mathrm{MoS}_{2}$ lattice forms an unfavorable environment for the $\mathrm{Pb}$ atoms in these nanostructures. In order to elucidate preliminary the accommodation type, chemical bonding and the highest possible (at equilibrium) concentration of $\mathrm{Pb}$ atoms in the samples of $\mathrm{MoS}_{2}$ nanotubes a set of DFT calculations were performed. Two cases were considered: (1) the 
lead atoms substitute the Mo in the lattice and serve as dopants ( $\mathrm{Mo}_{1-x} \mathrm{~Pb}_{x} \mathrm{~S}_{2}$ solid solutions); (2) the $\mathrm{Pb}$ atoms intercalate into the interlayer space of the $\mathrm{MoS}_{2}$ host lattice ( $\mathrm{Pb}_{y} / \mathrm{MoS}_{2}$ intercalates).

\subsubsection{Electronic Structure}

Test DFT calculations at LDA level were performed on example of the bulk $2 \mathrm{H}-\mathrm{MoS}_{2}$ and fcc-PbS crystals (see Section 3). $2 \mathrm{H}-\mathrm{MoS}_{2}$ was found to be a semiconductor with direct band gap $2.18 \mathrm{eV}$ and indirect $\Gamma \rightarrow \frac{1}{2} \mathrm{~K} \Gamma$ gap of $0.59 \mathrm{eV}$. The valence band is composed mainly of S3p-states, about $\sim 3.5-6.0 \mathrm{eV}$ below the Fermi level. The top of the valence band and the bottom of the conduction band are dominated by Mo4d-states. In the electronic structure of fcc-PbS the occupied $\mathrm{Pb} 6 \mathrm{~s}$ band is present between 6.5 and $9.5 \mathrm{eV}$ below the Fermi level. The three predominately S3p bands are located between -6 and $0 \mathrm{eV}$. The fundamental band gap of $0.11 \mathrm{eV}$ is direct at the $\mathrm{L}$ point with the conduction band composed predominately of Pb6 $p$ states. While the values of the band gaps are also typically underestimated in LDA approach, the band structures are in full accordance with other theoretical and experimental results $[21,22]$.

In general, the band structure of $\mathrm{MoS}_{2}$ and the corresponding picture of the densities of states (DOS) are essentially not perturbed by substitutional doping of the Mo sublattice by a single $\mathrm{Pb}$ atom (Figure 5a). A single level separates from the bottom of the conduction band into the band gap of pristine $\mathrm{MoS}_{2}$, while the Fermi energy shifts downwards the top of valence band. In addition, a new level emerges at $1 \mathrm{eV}$ below the bottom of the valence band of doped $\mathrm{MoS}_{2}$. It is composed mainly of Pb6s states, which do not overlap with the $\mathrm{S} 3 p$ band and do not contribute to the chemical bonding of the system. Thus, like in the case of $\mathrm{PbS}$ compound, the chemical bonding of $\mathrm{Pb}$ atom with $\mathrm{S}$ atoms is released due to the depopulation of only $\mathrm{Pb} 6 p$ states, which can be found at 5-6 eV above the Fermi energy. All these features are evidence for a p-type semiconducting character of the $\mathrm{Pb}$-doped $\mathrm{MoS}_{2}$ and nominal $2+$ oxidation state of $\mathrm{Pb}$. Such oxidation state and the arising electron deficiency of $\mathrm{MoS}_{2}$ electronic structure should lead to destabilization of the chemical environment of the $\mathrm{Pb}$-doped $\mathrm{MoS}_{2}$ lattice. The map of the differential electron densities for this system supports this deduction and demonstrates a vanishing electron density and weakening of the bonds near the $\mathrm{Pb}$ atom compared to the electron density nearby the Mo atoms (Figure 5a).

Another possible process, which could occur during the $\mathrm{Pb}$-promoted growth of $\mathrm{MoS}_{2}$ nanotubes, is an intercalation of $\mathrm{MoS}_{2}$ lattice by $\mathrm{Pb}$ atoms. The intercalation by a single $\mathrm{Pb}$ atom also does not affect the band structure essentially (Figure 5b). Yet, in this case the Fermi level shifts upwards into the conduction band. The localized $\mathrm{Pb} 6 s$ states occur deeper at $2 \mathrm{eV}$ below the bottom of valence band. $\mathrm{Pb} 6 p$ states of the intercalating $\mathrm{Pb}$ atom are more delocalized within the conduction band, than in the aforementioned case of $\mathrm{Pb}$ doping. Thus, $\mathrm{a} \mathrm{Pb}_{y} / \mathrm{MoS}_{2}$ intercalate should behave as an n-type semiconductor. The map of differential electron density for this system does not reveal any essential covalent bond formation with $\mathrm{S}$ atoms and serves as an evidence for the non-amicable environment of the intercalating $\mathrm{Pb}$ atoms. 
Figure 5. (a) Band structure; electronic partial densities of states (DOS) for $\mathrm{Pb}$ and differential electron density map for $2 \mathrm{H}-\mathrm{MoS}_{2}$ crystal doped by single $\mathrm{Pb}$ atom; (b) Band structure, DOS for $\mathrm{Pb}$ and differential electron density map for $2 \mathrm{H}-\mathrm{MoS}_{2}$ crystal intercalated by single $\mathrm{Pb}$ atom. LDA DFT calculations.
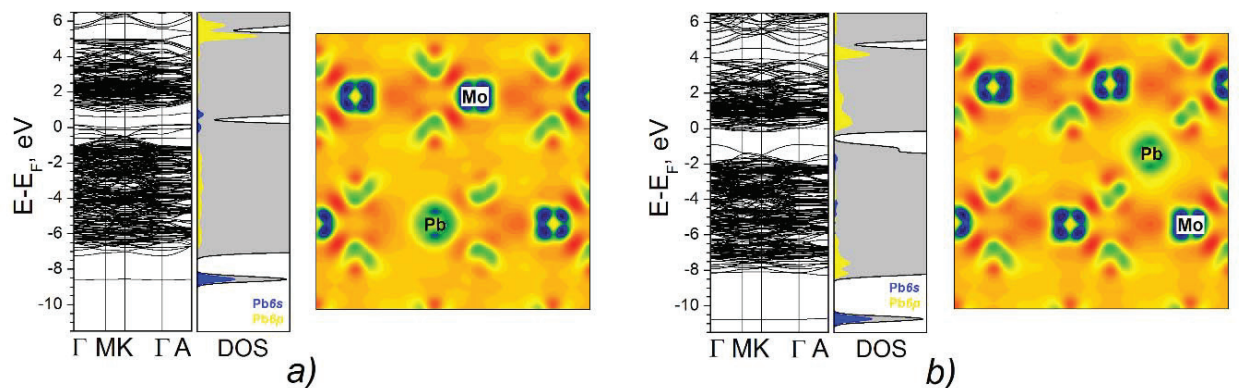

b)

\subsubsection{Estimation of the Stability Limit for Lead Atoms in the $\mathrm{MoS}_{2}$ Lattices}

Both doping and intercalation by single $\mathrm{Pb}$ atoms lead to a destabilization of the $\mathrm{MoS}_{2}$ electronic system. A weakening of the chemical bonding within the $\mathrm{MoS}_{2}$ lattice due to electron deficiency of the valence band or occupation of anti-bonding Mo $4 d$-states, are observed, respectively. A stabilization of the lattice is not favored also by the sterical strain induced after the difference in the radii between $\mathrm{Mo}$ and $\mathrm{Pb}$ atoms. e.g., optimized lattice parameters for substitutionally $\mathrm{Pb}$-doped $\mathrm{MoS}_{2}$ lattice reveal a slight increase of the lattice parameter $a$ from $3.12 \AA$ to $3.15 \AA$ due to the single $\mathrm{Pb}$ impurity atom and up to $3.19 \AA$ due to the "cluster" of four $\mathrm{Pb}$ atoms. The interlayer distance is not considerably affected in both cases and is $\sim 0.05 \AA$ smaller, than in the pure $2 \mathrm{H}-\mathrm{MoS}_{2}$. The intercalation of $\mathrm{MoS}_{2}$ lattice by individual $\mathrm{Pb}$ atoms has opposite effect: the $a$ lattice parameter is increased by $\sim 0.01 \AA$, while the interlayer distance is getting larger by $\sim 0.4 \AA$. These trends agree well with the large atomic radius of $\mathrm{Pb}$ atoms. Indeed, the calculated metal-sulfur bond lengths in the bulk of $\mathrm{PbS}$ and $\mathrm{MoS}_{2}$ are: $2.94 \AA$ for $\mathrm{Pb}-\mathrm{S}$ and $2.40 \AA$ for Mo-S, while the length of the covalent $\mathrm{Pb}-\mathrm{S}$ bond within $\mathrm{Pb}$-doped $\mathrm{MoS}_{2}$ lattice is $2.65 \AA$.

Furthermore, the influence of the concentration and ordering of the impurity atoms on the thermodynamic stability of doped $\mathrm{Mo}_{1-x} \mathrm{~Pb}_{x} \mathrm{~S}_{2}$ and intercalated $\mathrm{Pb}_{y} / \mathrm{MoS}_{2}$ phases can be considered. To characterize the stability of $\mathrm{Mo}_{1-x} \mathrm{~Pb}_{x} \mathrm{~S}_{2}$ and the $\mathrm{Pb}_{y} / \mathrm{MoS}_{2}$ phases, the cohesion energies $E_{\text {coh }}$ were calculated for a set of $4 \times 4 \times 12 \mathrm{H}-\mathrm{MoS}_{2}$ supercells modified by 1-4 Mo atoms (Table 1). In agreement with the picture of the electronic structure, the absolute values of $E_{\mathrm{coh}}$ for all modified systems vanish with the growing content of $\mathrm{Pb}$, which is an evidence for the weakening of chemical bonding in both doped and intercalated $\mathrm{MoS}_{2}$ compared to the pristine $\mathrm{MoS}_{2}$. Noteworthy, the cohesion energies of the solid solutions as well as intercalates vary almost in the same order of magnitude, and the competing formation of both phases during the $\mathrm{Pb}$-promoted growth of $\mathrm{MoS}_{2}$ nanotubes can be anticipated.

The exact growth mechanism and the main compounds participating in the growth of $\mathrm{MoS}_{2}$ nanotubes have still to be found. Yet, a first insight in this process is possible by the consideration of some model reactions. The formation energies $\Delta E$ for $\mathrm{Mo}_{1-x} \mathrm{~Pb}_{x} \mathrm{~S}_{2}$ and $\mathrm{Pb} / \mathrm{MoS}_{2}$ phases from $\mathrm{MoS}_{2}$ 
and the corresponding compounds were estimated using calculated change in the total energies of the next formal reactions:

$$
\begin{gathered}
(1-x) \mathrm{MoS}_{2}+x \mathrm{PbS}+x \mathrm{~S}=\mathrm{Mo}_{1-x} \mathrm{~Pb}_{x} \mathrm{~S}_{2} \\
y \mathrm{~Pb}+\mathrm{MoS}_{2}=\mathrm{Pb}_{y} / \mathrm{MoS}_{2}
\end{gathered}
$$

Both reactions have been found to be highly endothermic (Table 2). Concerning the calculated values of $\Delta E$, a strong tendency for the phase separation of $\mathrm{Pb}$-modified $\mathrm{MoS}_{2}$ lattice into a mixture of simple binary sulfides and simple elements may be contemplated. These theoretical observations are in agreement with the experimental finding of the time-depended $\mathrm{Pb}$ content in fabricated $\mathrm{MoS}_{2}$ samples.

Table 2. Cohesion energies $E_{\text {coh }}$ and energies of formation $\Delta E$ of $\mathrm{Mo1}_{-x} \mathrm{~Pb}_{x} \mathrm{~S}_{2}$ solid solutions and $\mathrm{Pb}_{y} / \mathrm{MoS}_{2}$ intercalates concerning reactions (1) and (2), as a function of the content

\begin{tabular}{|c|c|c|c|c|}
\hline \multirow{2}{*}{ Isomer } & Pb atoms Arrangement & Supercell Composition & $E_{\text {coh, }} \mathrm{eV} /$ atom & $\Delta E, \mathrm{eV} / \mathrm{Pb}$ \\
\hline & no $\mathrm{Pb}$ atoms & $\mathrm{Mo}_{32} \mathrm{~S}_{64}, x, y=0.00$ & -1.58 & - \\
\hline \multicolumn{5}{|c|}{ Mo1-x $_{1} \mathrm{~Pb}_{x} \mathrm{~S}_{2}$ Doped by } \\
\hline D1 & single $\mathrm{Pb}$ atom & $\mathrm{Pb}_{1} \mathrm{Mo}_{31} \mathrm{~S}_{64}, x=0.031$ & -1.50 & 4.65 \\
\hline $\mathrm{D} 2 \mathrm{a}$ & $\begin{array}{l}\text { two neighbor } \mathrm{Pb} \text { atoms } \\
\text { within the same layer }\end{array}$ & $\mathrm{Pb}_{2} \mathrm{Mo}_{30} \mathrm{~S}_{64}, x=0.063$ & -1.45 & 3.75 \\
\hline $\mathrm{D} 2 \mathrm{~b}$ & $\begin{array}{l}\text { two distant } \mathrm{Pb} \text { atoms } \\
\text { within the same layer }\end{array}$ & $\mathrm{Pb}_{2} \mathrm{Mo}_{30} \mathrm{~S}_{64}, x=0.063$ & -1.43 & 4.63 \\
\hline $\mathrm{D} 2 \mathrm{c}$ & $\begin{array}{c}\text { two neighbor } \mathrm{Pb} \text { atoms in } \\
\text { different layers }\end{array}$ & $\mathrm{Pb}_{2} \mathrm{Mo}_{30} \mathrm{~S}_{64}, x=0.063$ & -1.43 & 4.42 \\
\hline D2d & $\begin{array}{l}\text { two distant } \mathrm{Pb} \text { atoms in } \\
\text { different layers }\end{array}$ & $\mathrm{Pb}_{2} \mathrm{Mo}_{30} \mathrm{~S}_{64}, x=0.063$ & -1.43 & 4.46 \\
\hline D3a & $\begin{array}{l}\text { three neighbor } \mathrm{Pb} \text { atoms } \\
\text { within the same layer }\end{array}$ & $\mathrm{Pb}_{3} \mathrm{Mo}_{29} \mathrm{~S}_{64}, x=0.094$ & -1.39 & 3.34 \\
\hline $\mathrm{D} 4 \mathrm{a}$ & $\begin{array}{c}\text { four neighbor } \mathrm{Pb} \text { atoms } \\
\text { within the same layer }\end{array}$ & $\mathrm{Pb}_{4} \mathrm{Mo}_{28} \mathrm{~S}_{64}, x=0.125$ & -1.33 & 3.18 \\
\hline \multicolumn{5}{|c|}{$\mathrm{Pb}_{y} / \mathrm{MoS}_{2}$ Intercalated by } \\
\hline I1 & single $\mathrm{Pb}$ atom & $\mathrm{Pb}_{1} / \mathrm{Mo}_{32} \mathrm{~S}_{64}, y=0.031$ & -1.51 & 5.72 \\
\hline $\mathrm{I} 2 \mathrm{a}$ & $\begin{array}{l}\text { two neighbor } \mathrm{Pb} \text { atoms } \\
\text { within the same vdW gap }\end{array}$ & $\mathrm{Pb}_{2} / \mathrm{Mo}_{32} \mathrm{~S}_{64}, y=0.063$ & -1.47 & 3.79 \\
\hline $\mathrm{I} 2 \mathrm{~b}$ & $\begin{array}{c}\text { two distant } \mathrm{Pb} \text { atoms } \\
\text { within the same vdW gap }\end{array}$ & $\mathrm{Pb}_{2} / \mathrm{Mo}_{32} \mathrm{~S}_{64}, y=0.063$ & -1.46 & 4.28 \\
\hline $\mathrm{I} 2 \mathrm{c}$ & $\begin{array}{l}\text { two neighbor } \mathrm{Pb} \text { atoms in } \\
\text { different vdW gaps }\end{array}$ & $\mathrm{Pb}_{2} / \mathrm{Mo}_{32} \mathrm{~S}_{64}, y=0.063$ & -1.44 & 5.66 \\
\hline $\mathrm{I} 2 \mathrm{~d}$ & $\begin{array}{l}\text { two distant } \mathrm{Pb} \text { atoms in } \\
\text { different vdW gaps }\end{array}$ & $\mathrm{Pb}_{2} / \mathrm{Mo}_{32} \mathrm{~S}_{64}, y=0.063$ & -1.43 & 5.73 \\
\hline
\end{tabular}
and the arrangement of $\mathrm{Pb}$ atoms within model supercells. LDA DFT calculations.

Noteworthy, the formation energies for the model supercells with "associated" (neighboring) $\mathrm{Pb}$ atoms in both $\mathrm{Pb}$-doped and $\mathrm{Pb}$-intercalated $\mathrm{MoS}_{2}$ are considerably lower, than for those supercells, where all $\mathrm{Pb}$ atoms are separated. e.g., the interaction between two $\mathrm{Pb}$ atoms separated by 
the distance $\sim 6.3 \AA$ in $\mathrm{Mo}_{1-x} \mathrm{~Pb}_{x} \mathrm{~S}_{2}$ solid solutions is almost absent ( $\Delta E$ for $\mathrm{D} 2 \mathrm{~b}$ and D2c isomers are close to that of $\mathrm{D} 1$, Table 1). The same tendency can be obtained in $\mathrm{Pb}_{y} / \mathrm{MoS}_{2}$ intercalates, yet, with a higher range of interaction between $\mathrm{Pb}$ atoms ( $\Delta E$ for I2b isomer is still less, than for I1, Table 1). Thus, the solid solutions of $\mathrm{Mo}_{-x} \mathrm{~Pb}_{x} \mathrm{~S}_{2}$ should be more stable than $\mathrm{Pb}_{y} / \mathrm{MoS}_{2}$ intercalates, since the coalescence of intercalated $\mathrm{Pb}$ atoms is more favorable and $\mathrm{Pb} / \mathrm{MoS}_{2}$ intercalates might exist in the narrower part of the phase diagram at a lower $\mathrm{Pb}$ content, than $\mathrm{Mo1}_{-} \mathrm{Pb} \mathrm{P}_{x} \mathrm{~S}_{2}$ solid solutions.

Concerning the analysis of calculated formation energies for the case of single doping or intercalating $\mathrm{Pb}$ atoms, the formation of $\mathrm{Mo}_{1-x} \mathrm{~Pb}_{x} \mathrm{~S}_{2}$ solid solutions is more likely, than the formation of $\mathrm{Pb}_{y} / \mathrm{MoS}_{2}$ intercalates. As well, the values of formation energies allow an estimation of the limit of the lead solubility at certain temperature $\mathrm{T}$ at thermodynamic equilibrium. As example, we consider roughly the possible content within $\mathrm{Mol}_{-} \mathrm{Pb}_{x} \mathrm{~S}_{2}$ compounds after reaction (1). The preference of one of two states in a chemical reaction is determined by the free energy change $\Delta F=\Delta U-T \Delta S$, where $\Delta U$ is the change of internal energy, $\Delta S$ is the change of entropy. The condition corresponding to the phase separation is $\Delta F \leq 0$.

In the first approximation, $\Delta \mathrm{S}$ can be defined as the configurational entropy of randomly distributed $\mathrm{Pb}$ atoms in the metal sublattice of $\mathrm{Mo}_{1-x} \mathrm{~Pb}_{x} \mathrm{~S}_{2}$. From the theory of ideal solutions it follows, that

$$
\Delta S=R\left(\ln x+\frac{(1-x)}{x} \ln (1-x)\right)
$$

where $\Delta S$ is expressed per mole of $\mathrm{Pb} . \Delta U$ can be approximated as the energy of reaction (1) $(\Delta U=\Delta E)$. The estimation of the free energy change for the phase separation within $\mathrm{Mo}_{1-x} \mathrm{~Pb}_{x} \mathrm{~S}_{2}$ solid solutions with a fortiori low $x$ imply the use of the energy for the formation of single $\mathrm{Pb}$ atom within $\mathrm{MoS}_{2}$ lattice, i.e., $\Delta E=4.65 \mathrm{eV}$ or $448.7 \mathrm{~kJ} / \mathrm{Pb}$-mol (isomer D1, Table 1).

The results of this approach based on the formal reaction (1) are visualized in Figure 6. They reveal that the substitution of $\mathrm{Pb}$ atom instead of the Mo atom within the $\mathrm{MoS}_{2}$ lattice is a quite rare event and the content of $\mathrm{Pb}$ at thermodynamic equilibrium would be around $x=10^{-7}$ at the temperatures $\sim 3000 \mathrm{~K}$. In this manner, the experimentally fabricated $\mathrm{MoS}_{2}$ nanotube samples with primary $\mathrm{Pb}$ content of $x=0.12$, which were formed during the extreme reaction conditions of the sun-light driven evaporation, are thermodynamically unstable and should show a high urge towards a phase separation.

Figure 6. Calculated free energy $\Delta F$ of the phase separation within $\mathrm{Mo}_{1-x} \mathrm{~Pb}_{x} \mathrm{~S}_{2}$ solid solutions depending on the temperature and $\mathrm{Pb}$ content $(x)$.

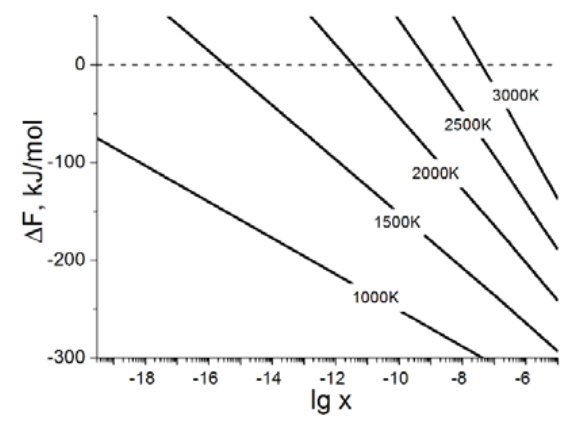




\section{Experimental Section}

$\mathrm{MoO}_{3-x}$ nanowhiskers and $\mathrm{MoS}_{2}$ nanotubes were prepared using solar ablation system [15]. The $\mathrm{Pb}$ and $\mathrm{MoS}_{2}$ mixture was sealed in a quartz ampoule and irradiated using solar ablation system for $30 \mathrm{~s}$ in the case of $\mathrm{MoO}_{3-x}$ nanowhiskers, and for $10 \mathrm{~min}$ in the case of $\mathrm{MoS}_{2}$ nanotubes. Both nanostructures contain a few atomic percent of $\mathrm{Pb}$. After spending a year in ambient conditions these samples were annealed at $810{ }^{\circ} \mathrm{C}$ in the presence of $\mathrm{H}_{2} \mathrm{~S}$ and forming gas $\left(5 \% \mathrm{H}_{2}\right.$ and $\left.95 \% \mathrm{~N}_{2}\right)$. The annealing time varied from $30 \mathrm{~min}$ to $2 \mathrm{~h}$. The sample labeling can be seen in Table 2 .

At each stage the samples were examined with a transmission electron microscope (TEM) operating at $120 \mathrm{kV}$, equipped with an energy-dispersive X-ray spectroscopy (EDS) detector for chemical analysis. The SEM image was generated using a scanning electron microscope (SEM), and high-resolution imaging was achieved with a field-emission gun TEM operating at $300 \mathrm{kV}$.

The calculations were performed using the SIESTA 2.0 (Madrid, Spain) implementation [23,24] within the framework of density-functional theory (DFT) [25]. The exchange-correlation potential within the local-density approximation (LDA) with the Perdew-Zunger parametrization was used [26]. The core electrons were treated within the frozen core approximation, applying norm-conserving Troullier-Martins pseudopotentials [27]. The valence electrons were taken as $3 s^{2} 3 p^{4}$ for $\mathrm{S}, 4 d^{5} 5 s^{1} 5 p^{0}$ for $\mathrm{Mo}, 6 s^{2} 6 p^{2}$ for $\mathrm{Pb}$, while $\mathrm{Pb} 5 d^{10}$ electrons were included as semi-core state. The pseudopotential core radii were chosen, as suggested by Martins, and are equal to $1.70 \mathrm{a}_{\mathrm{B}}$ for all S states, $2.45 \mathrm{a}_{\mathrm{B}}$ for Mo4d and Mo5s, 2.65 ав for Mo5p states, 3.20 ав for Pb6s and Pb6 $p, 3.00$ ав for Pb5d states. In all calculations double- $\zeta$ basis set is used for all atoms. For $k$-point sampling, a cutoff of $10 \AA$ was used [28]. The $k$-point mesh was generated by the method of Monkhorst and Pack [29]. The real-space grid used for the numeric integrations was set to correspond to the energy cutoff of 300 Ry. For the study of substitutional doping and intercalation of $\mathrm{MoS}_{2}$ by $\mathrm{Pb}$ atoms the supercell of $4 \times 4 \times 1$ of $2 \mathrm{H}-\mathrm{MoS}_{2}$ unit cells was considered. The energies of chemical reactions were estimated from the calculations of total energies for $2 \mathrm{H}-\mathrm{MoS}_{2}$, bcc-Mo, fcc- $\mathrm{Pb}$, fcc- $\mathrm{PbS}$ and molecular S8. All calculations were performed using variable-cell and atomic position relaxation, with convergence criteria set to correspond to a maximum residual stress of $0.1 \mathrm{GPa}$ for each component of the stress tensor, and maximum residual force component of $0.01 \mathrm{eV} / \AA$.

\section{Conclusions}

The experimental work shows that $\mathrm{MoO}_{3-x}$ (1) nanowhiskers and $\mathrm{MoS}_{2}$ (1) nanotubes synthesized using solar ablation system, are stable for more than a year in the ambient conditions. However, $\mathrm{Pb}$ atoms that were observed in these nanostructures, are not stable in high concentration and tend to diffuse out of the lattice. The $\mathrm{Pb}$ outdiffusion from these nanostructures did not influence their high-temperature stability and their conversion into $\mathrm{MoS}_{2}(3,4)$ nanotubes. In all cases $\mathrm{MoS}_{2}$ nanotubes were observed after the sulfidization and annealing of $\mathrm{MoO}_{3-x}(2)$ nanowhiskers and $\mathrm{MoS}_{2}(2)$ nanotubes. According to the EDS measurements the initial $\mathrm{Pb}$ concentration in both nanostructures was reduced after one year in the drawer. While subsequent annealing of these nanostructures lead to additional reduction of their $\mathrm{Pb}$ concentration. It can be seen from the EDS spectra that in the case of molybdenum suboxide nanowhiskers $\mathrm{MoO}_{3-x}(2)$ as a precursor material, the $\mathrm{Pb}$ peak disappears after two hours 
$\left(\mathrm{H}_{2} \mathrm{~S}\right)$ annealing, while the same peak disappears after $30 \mathrm{~min}$ annealing at $810^{\circ} \mathrm{C}$ in the case of $\mathrm{MoS}_{2}(2)$ nanotubes.

DFT calculations have verified a very low affinity of $\mathrm{Pb}$ atoms as either substitutional or intercalating agents to the host $\mathrm{MoS}_{2}$ lattice. Both types of modifications should lead to the destabilization of the electronic structure of the pristine $\mathrm{MoS}_{2}$ and cause a weak chemical bonding between $\mathrm{Pb}$ and $\mathrm{S}$ atoms with the oxidation state of $\mathrm{Pb}^{2+}$. The calculations have also demonstrated that the interaction between $\mathrm{Pb}$ atoms as intercalates is slightly stronger, than the interaction between $\mathrm{Pb}$ atoms as substitutional dopants in the $\mathrm{MoS}_{2}$ lattice. Thus, $\mathrm{Pb}_{y} / \mathrm{MoS}_{2}$ intercalates might exist only at lower $\mathrm{Pb}$ content compared to a solid solution of $\mathrm{Mo}_{1-x} \mathrm{~Pb}_{x} \mathrm{~S}_{2}$. The latter phase seems to be a more favorite form of $\mathrm{Pb}$ within the fabricated $\mathrm{MoS}_{2}$ nanotubes. The estimations of the free energies for the phase separation of $\mathrm{Mo1}_{-x} \mathrm{~Pb}_{x} \mathrm{~S}_{2}$ solid solutions explain the experimentally observed time- and high-temperature reduction of the $\mathrm{Pb}$ content in the nanotube lattice. The experimentally reached $\mathrm{Pb}$ concentrations $(x=0.3)$ may be attributed to a thermodynamically unstable system which was obtained by a fast kinetic of the chemical reaction between lead atoms and the primary $2 \mathrm{H}-\mathrm{MoS}_{2}$ lattice during the solar ablation experiments.

\section{Acknowledgments}

We wish to thank Jeffrey M. Gordon and Daniel Feuerman from the Jacob Blaustein Institutes for Desert Research in Sede Boqer of the Ben-Gurion University for their assistance in the synthesis of the oxide nanowhisekrs and nanotubes. This research was supported by the ERC grant INTIF 226639; the EU ITN 317451 grant and a grant of the Israel Science Foundation. Reshef Tenne acknowledges the support of the Harold Perlman Foundation and the Irving and Azelle Waltcher Foundation in honor of Moshe Levy. We are grateful also to the Irving and Cherna Moskowitz Center for Nano and BioNano Imaging. Reshef Tenne holds the Drake Family chair in Nanotechnology and is the director of the Helen and Martin Kimmel Center for Nanoscale Science.

\section{Author Contributions}

Olga Brontvein and Reshef Tenne designed the research. Olga Brontvein recorded and analyzed TEM, SEM and EDS data. Andrey Enyashin carried out DFT calculations. The manuscript was written through contributions of all authors. All authors have given approval to the final version of the manuscript.

\section{Conflicts of Interest}

The authors declare no conflict of interest.

\section{References}

1. Kaplan-Ashiri, I.; Cohen, S.; Gartsman, K.; Rosentsveig, R.; Seifert, G.; Tenne, R. Mechanical behavior of individual $\mathrm{WS}_{2}$ nanotubes. J. Mater. Res. 2004, 19, 454-459. 
2. Kaplan-Ashiri, I.; Cohen, S.R.; Gartsman, K.; Ivanovskaya, V.; Heine, T.; Seifert, G.; Wiesel, I.; Wagner, H.; Tenne, R. On the mechanical behavior of $\mathrm{WS}_{2}$ nanotubes under axial tension and compression. Proc. Natl. Acad. Sci. USA 2006, 103, 523.

3. Nagapriya, K.S.; Goldbart, O.; Kaplan-Ashiri, I.; Seifert, G.; Tenne, R.; Joselevich, E. Torsional stick-slip behavior in $\mathrm{WS}_{2}$ nanotubes. Phys. Rev. Lett. 2008, 101, 195501.

4. Frey, G.L.; Tenne, R.; Matthews, M.J.; Dresselhaus, M.S.; Dresselhaus, G. Optical properties of $M_{2}(M=\mathrm{Mo}, \mathrm{W})$ inorganic fullerenelike and nanotube material optical absorption and resonance raman measurements. J. Mater. Res. 1998, 13, 2412-2417.

5. Staiger, M.; Rafailov, P.; Gartsman, K.; Telg, H.; Krause, M.; Radovsky, G.; Zak, A.; Thomsen, C. Excitonic resonances in WS2 nanotubes. Phys. Rev. B 2012, 86, doi:10.1103/PhysRevB.86.165423.

6. Virsek, M.; Jesih, A.; Milosevic, I.; Damnjanovic, M.; Remskar, M. Raman scattering of the $\mathrm{MoS}_{2}$ and $\mathrm{WS}_{2}$ single nanotubes. Surf. Sci. 2007, 601, 2868-2872.

7. Enyashin, A.; Gemming, S.; Seifert, G. Nanosized allotropes of molybdenum disulfide. Eur. Phys. J.-Spec. Top. 2007, 149, 103-125.

8. Levi, R.; Bitton, O.; Leitus, G.; Tenne, R.; Joselevich, E. Field-effect transistors based on $\mathrm{WS}_{2}$ nanotubes with high current-carrying capacity. Nano Lett. 2013, 13, 3736-3741.

9. Remskar, M.; Skraba, Z.; Cleton, F.; Sanjines, R.; Levy, F. MoS2 as microtubes. Appl. Phys. Lett. 1996, 69, 351-353.

10. Remskar, M.; Mrzel, A.; Virsek, M.; Godec, M.; Krause, M.; Kolitsch, A.; Singh, A.; Seabaugh, A. The $\mathrm{MoS}_{2}$ nanotubes with defect-controlled electric properties. Nanoscale Res. Lett. 2010, $6,26$.

11. Yella, A.; Mugnaioli, E.; Panthöfer, M.; Therese, H.A.; Kolb, U.; Tremel, W. Bismuth-catalyzed growth of $\mathrm{SnS}_{2}$ nanotubes and their stability. Angew. Chem. Int. Ed. 2009, 48, 6426-6430.

12. Radovsky, G.; Popovitz-Biro, R.; Stroppa, D.G.; Houben, L.; Tenne, R. Nanotubes from chalcogenide misfit compounds: $\mathrm{Sn}-\mathrm{S}$ and $\mathrm{Nb}-\mathrm{Pb}-\mathrm{S}$. Acc. Chem. Res. 2013, 47, 406-416.

13. Radovsky, G.; Popovitz-Biro, R.; Staiger, M.; Gartsman, K.; Thomsen, C.; Lorenz, T.; Seifert, G.; Tenne, R. Synthesis of copious amounts of $\mathrm{SnS}_{2}$ and $\mathrm{SnS}_{2} / \mathrm{SnS}$ nanotubes with ordered superstructures. Angew. Chem. Int. Ed. 2011, 50, 12316-12320.

14. Zak, A.; Sallacan-Ecker, L.; Margolin, A.; Genut, M.; Tenne, R. Insight into the growth mechanism of $\mathrm{WS}_{2}$ nanotubes in the scaled-up fluidized bed reactor. Nano 2009, 4, 91-98.

15. Brontvein, O.; Stroppa, D.G.; Popovitz-Biro, R.; Albu-Yaron, A.; Levy, M.; Feuerman, D.; Houben, L.; Tenne, R.; Gordon, J.M. New high-temperature Pb-catalyzed synthesis of inorganic nanotubes. J. Am. Chem. Soc. 2012, 134, 16379-16386.

16. Brontvein, O.; Jayaram, V.; Reddy, K.P.J.; Gordon, J.M.; Tenne, R. Two-step synthesis of MoS 2 nanotubes using shock waves with lead as growth promoter. Z. Anorg. Allg. Chem. 2014, 640, 1152-1158.

17. Feldman, Y.; Wasserman, E.; Srolovitz, D.; Tenne, R. High-rate, gas-phase growth of $\mathrm{MoS}_{2}$ nested inorganic fullerenes and nanotubes. Science 1995, 267, 222-225.

18. Yamazoe, N.; Kihlborg, L. $\mathrm{Mo}_{5} \mathrm{O}_{14}$-Twinning and three-dimensional structure, determined from a partly tantalum-substituted crystal. Acta Crystallogr. Sect. B 1975, 31, 1666-1672. 
19. Yamazoe, N.; Ekstrom, T.; Kihlborg, L. Structural effects of vanadium substitution in molybdenum oxide (Mo17O47). Acta Chem. Scand. Ser. A 1975, 29, 404-408.

20. Kihlborg, L. Stabilization of the tunnel structure of molybdenum oxide ( $\left.\mathrm{Mo}_{5} \mathrm{O}_{14}\right)$ by partial metal atom substitution. Acta Chem. Scand. 1969, 23, 1834-1835.

21. Boker, T.; Severin, R.; Muller, A.; Janowitz, C.; Manzke, R.; Voss, D.; Kruger, P.; Mazur, A.; Pollmann, J. Band structure of $\mathrm{MoS}_{2}, \mathrm{MoSe}_{2}$, and $\alpha-\mathrm{MoTe}_{2}$ : Angle-resolved photoelectron spectroscopy and ab initio calculations. Phys. Rev. B 2001, 64, 235305.

22. Walsh, A. Effects of reduced dimensionality on the electronic structure and defect chemistry of semiconducting hybrid organic-inorganic PbS solids. Proc. R. Soc. A 2011, 467, 1970-1985.

23. Ordejon, P.; Artacho, E.; Soler, J.M. Self-consistent order-N density-functional calculations for very large systems. Phys. Rev. B 1996, 53, 10441-10444.

24. Soler, J.M.; Artacho, E.; Gale, J.D.; Garcia, A.; Junquera, J.; Ordejon, P.; Sanchez-Portal, D. The SIESTA method for ab initio order-N materials simulation. J. Phys.-Condens. Matter 2002, 14, 2745-2779.

25. Hohenberg, P.; Kohn, W. Inhomogeneous electron gas. Phys. Rev. 1964, 136, B864-B871.

26. Perdew, J.P.; Zunger, A. Self-interaction correction to density-functional approximations for many-electron systems. Phys. Rev. B 1981, 23, 5048-5079.

27. Troullier, N.; Martins, J.L. Efficient pseudopotentials for plane-wave calculations. Phys. Rev. $B$ 1991, 43, 1993-2006.

28. Moreno, J.; Soler, J.M. Optimal meshes for integrals in real-space and reciprocal-space unit cells. Phys. Rev. B 1992, 45, 13891-13898.

29. Monkhorst, H.J.; Pack, J.D. Special points for Brillouin-zone integrations. Phys. Rev. B 1976, 13, 5188-5192. 


\title{
Microstructural Study of IF-WS $\mathbf{2}_{\mathbf{2}}$ Failure Modes
}

\author{
Jamie Cook, Steven Rhyans, Lou Roncase, Garth Hobson and Claudia C. Luhrs
}

\begin{abstract}
This manuscript summarizes the failure mechanisms found in inorganic fullerene-type tungsten disulfide $\left(\mathrm{IF}-\mathrm{WS}_{2}\right)$ nanoparticles treated with diverse pressure loading methods. The approaches utilized to induce failure included: the use of an ultrasonic horn, the buildup of high pressures inside a shock tube which created a shock wave that propagated and impinged in the sample, and impact with military rounds. After treatment, samples were characterized using electron microscopy, powder X-ray diffraction, energy dispersive X-ray spectroscopy, and surface area analysis. The microstructural changes observed in the IF-WS 2 particulates as a consequence of the treatments could be categorized in two distinct fracture modes. The most commonly observed was the formation of a crack at the particles surface followed by a phase transformation from the 3D cage-like structures into the 2D layered polymorphs, with subsequent agglomeration of the plate-like sheets to produce larger particle sizes. The secondary mechanism identified was the incipient delamination of IF-WS2. We encountered evidence that the IF-WS 2 structure collapse initiated in all cases at the edges and vertices of the polyhedral particles, which acted as stress concentrators, independent of the load application mode or its duration.
\end{abstract}

Reprinted from Inorganics. Cite as: Cook, J.; Rhyans, S.; Roncase, L.; Hobson, G.; Luhrs, C.C. Microstructural Study of IF-WS 2 Failure Modes. Inorganics 2014, 2, 377-395.

\section{Introduction}

The first inorganic fullerene-like nanoparticles of $\mathrm{WS}_{2}$ were discovered by Tenne et al., working at the Weizmann Institute of Science in 1992 [1]. Using the diffusion-controlled sulfurization of metal oxides, they were able to empirically prove the existence of inorganic compounds based on $\mathrm{WS}_{2}$ with structures that were believed to only exist for carbon-based materials. Shortly thereafter, the discovery of $\mathrm{WS}_{2}$ nanotubes and fullerene-like structures led to the establishment of a new field of inorganic chemistry; one dealing with closed-hollow nanomaterials [2-5].

Originally, carbon fullerenes, made of concentric layers of carbon, were thought to provide outstanding tribological properties, and under low loads and high velocities they do [6,7]. However, due to their phase transition from graphite to diamond at high temperatures and pressures, they cannot entirely live up to the expectations that their structures suggest [8]. IF-WS2 particles have numerous properties similar to the carbon fullerenes, making them excellent solid lubricants; their extremely small size (in the nanometer scale) gives them the ability to fill imperfections in the lubricated material to effectively smooth the surface and prevent degradation. Due to their spherical shape, IFs are also said to act like nano-ball bearings, feature that allows them to roll rather than slide, performing better than other solid lubricants [4].

Currently, IF-WS 2 particulates are recognized for their potential not only as lubricants but also as structural nanocomposites and shock absorbers [9-15]. 
The impressive shock absorbing performance of IF-WS 2 and IF-MoS 2 structures were first reported by Zhu et al. [16,17]. Using uniaxial shockwave pressures up to $30 \mathrm{GPa}$ and studying the microstructural features of samples treated at diverse pressure settings, it was found that these cage-like particulates have superior performance than their carbon counterparts. Those reports also identified a lattice reduction for the samples treated at the higher pressures and provided a glimpse of the material breakage mechanisms. Moreover, those journal articles introduced the concept that smaller, more spherical IF particulates might be less prone to sustain damage than larger particles, a key feature to have in mind when designing highly resistant nanoparticles.

By studying the structural characteristics of a material that has fractured one can understand how materials fail and then make changes to the design and prevent, to a certain extent, the encountered failure modes. Fracture mechanics principles allow us to predict maximum working stress for a given material, establish relationships between materials properties, stress levels, crack producing flaws and conditions for the cracks propagation that ultimately will result in catastrophic breakdown of structures.

Here, we induced the breakage of IF-WS 2 particulate structures employing three different setups: (i) the use of an ultrasonic horn operated at diverse amplitudes and periods of time with the sample immersed in a solvent; (ii) pressure waves created inside a shock tube; and (iii) impact with military rounds. The ultrasonic horn was used at diverse amplitudes and treatment times and produced cyclic loading conditions similar to the ones used for fatigue studies of macroscopic objects. The shock tube and the military rounds produced a single uniaxial impact event over only fractions of a second.

The objective of the experiments was to study the microstructural characteristics of the materials postmortem and based on those observations identify failure modes - fracture mechanisms. Then establish relationships between those and the type and duration of each treatment method employed, identifying the most important variables for the particulates failure.

Our results demonstrated that the effects observed when applying pressure loads to the IF-WS2 nanometric particles are a particular case of the more general principles established by fracture mechanics to explain macroscopic crack propagation: stress concentrators at the particle surface play a much larger role in the material failure than the directionality or duration of methods used to apply the loads.

\section{Results and Discussion}

\subsection{Sample Characteristics before Treatment}

The IF-WS 2 particulates utilized for this study were analyzed by diverse techniques before treatment. TEM analysis showed that the IF nanostructures presented the typical hollow cores and the particles exhibit polyhedral shapes, as seen in Figure 1. The value for the lattice spacing observed varied depending on where the measurement was performed; the faces of the polyhedrons presented an average value of $0.62 \mathrm{~nm}$, while the vertices had an average separation of $0.63 \mathrm{~nm}$. Thus, when the IF particulates do not have a perfect spherical shape the lattice distance seem not to be uniform. Observation by SEM determined that the particle size distribution was between 30 and $420 \mathrm{~nm}$, with 
an average diameter of $143 \mathrm{~nm}$ and only a few particles of more than $500 \mathrm{~nm}$. BET surface area of the sample was determined to be $35 \mathrm{~m}^{2} / \mathrm{g}$.

Figure 1. TEM image of an IF-WS2 particle before treatment.

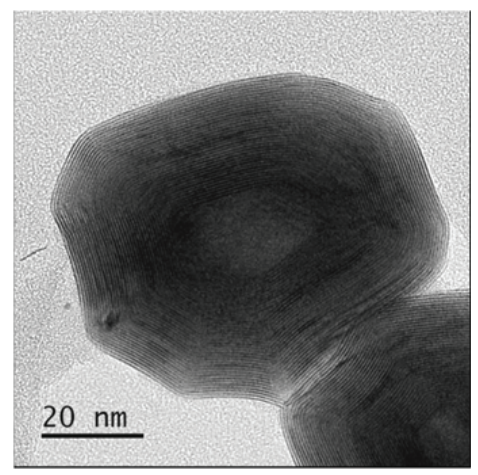

\subsection{Sample Postmortem Analysis}

Before presenting the post treatment sample characteristics, it is worth noting that the diverse methods used to impinge in the sample (each described in the experimental methods section) impart very different levels of energy at very different time scales:

It has been estimated that the use of military rounds of the size used at the velocities recorded provided approximately 2070 Joules in a non-isotropic single event that lasted only fractions of a second. The compressed-gas driven shock tube experiment produced a wave that first impacted in the Kevlar layer located in front of the sample (which was used solely to contain the powder IF-WS 2 ) impacting next the sample in a non-isotropic manner. This single incident also lasted fractions of a second. A pressure sensor located after the Kevlar layer recorded an average pressure of $1742 \mathrm{KPa}$ when using $\mathrm{He}$ as the gas, as such, the estimated pressure of the wave that actually reached the sample. In contrast, during the ultrasonic treatment, the particles were completely immersed in a liquid, thus, being subject to isotropic shocks for much longer periods of time (between 3,600 and $10,800 \mathrm{~s}$ ). The estimated energy conveyed when using a $1200 \mathrm{~W}$ ultrasound horn during such periods of time was from 4032 to 12,096 kilojoules respectively. In all cases, only IF-WS 2 particulates were tested, no composites are included in this study.

Some research groups have studied the variations in the $d_{002}$ interlayer spacing in the samples before and after pressure loads using XRD powder data or TEM observations to relate such values to unit cell expansions or contractions [17,18]. Given the dispersion in lattice values noted by TEM studies mentioned above in the untreated samples, the extraction of useful postmortem TEM data could result very complicated. The change in lattice constant for IF-WS 2 seen before treatment arises from the differences in curvature between different locations of the polyhedral cage-like spherical or semispherical IF-WS 2 nanoparticles. To determine if XRD patterns could provide more reliable data, given that they include information from all the crystal locations and orientations, we performed such analysis for the samples treated by the three loading methods. 
The XRD patterns of samples subjected to the diverse treatments are shown in Figure 2. The graph in the upper section of the image shows the peaks encountered between $5^{\circ}$ and $70^{\circ}(2 \theta)$, while the bottom image presents only the (002) peak. Specimens treated by ultrasound waves in wet conditions (dispersed in a solvent) and then dried for the analysis, present a (002) reflection that has shifted to higher angles. This alteration in the X-ray pattern indicates a reduction in the cell spacing when compared to the untreated sample. A similar observation was made in references above [17,18]. We believed that such shift could be related not only to a compressed IF cell but to a phase transition from the IF cage structure to a more dense $2 \mathrm{D}$ plate-like $\mathrm{WS}_{2}$ arrangement with slightly smaller 001 distance. The width of the (002) XRD peak for ultrasound treated powders in Figure 2 (red line) might suggest the presence of both, IF-WS 2 and two-dimensional $\mathrm{WS}_{2}$ in the same powder sample.

The peaks identified with the * symbol do not belong to $\mathrm{WS}_{2}$ but to $\mathrm{WO}_{3}$. The existence of small amounts of $\mathrm{WO}_{3}$ could not be verified by EDS analysis of large portions of the sample by SEM but was clearly identified by TEM of selected locations (uploaded as Figure S1).

By comparison, the samples collected after shock tube tests and military rounds insult show either no change or a slight shift to lower angles. Such result could be interpreted as no modification of the structure or a small expansion in the lattice parameter. We believe, however, that disparity in those results, when compared to the ultrasonic treated sample, might be more related to the experimental constrains faced when collecting the powders than a clear representation of the specimen features. The collection of the section of the sample that was directly exposed to the shockwave or to the impact of the penetrator was difficult; the particles had the tendency to get expelled from the impact site and only some of them remained in the center of the tested specimen or close to the hole left by the round and got recovered. That is, the XRD signal represents the average of all the particles gathered in the postmortem, some of which were not as affected by the event as the ones at the center of the impact location. In the case of ultrasonic treatment, the full sample was contained at all times and all particles were equally affected by the treatment, therefore rendering a powder that presented more uniform features and more reliable data, thus confirming a shift in the $d_{002}$.

The precise effects of applying diverse loading conditions in the specimens' microstructures were much more evident once the samples were studied by electron microscopy. We were able to conduct the analysis with smaller amounts of sample and use only particulates from affected locations. Figure 3 presents a SEM image of the IF-WS 2 particles before (a) and after (b) ultrasonic treatment. The existence of extended layered structures (identified with arrows) along with the original IF polyhedral, semispherical shapes is the most noticeable change. According to particle size distribution measurements (Figure 3c) the average diameter of the particles in the sample after treatment has shifted to larger sizes, $252 \mathrm{~nm}$ (blue histogram), and clearly shows a bimodal distribution. Particles between $800 \mathrm{~nm}$ and 1 micron, not seen in the untreated sample, are present in the ultrasonic treated specimen. The larger plate-like particulates correspond to the $2 \mathrm{D}$ polymorph and no longer present the hollow cage structure of IF-WS 2 . The appearance of the 2D phase supports the XRD data collected regarding both, the XRD peak shift and its width, and agrees with the initial reports suggesting a lattice compression $[17,18]$. Notably, the particles did not only collapsed and lose their hollow cores; they also transformed into 2D structures and then suffered an agglomeration mechanism that turned them into much larger, irregular shape units. 
Figure 2. X-ray diffraction analysis of particulates recovered after treatments. Top: reflections observed between $5^{\circ}$ and $70^{\circ}$; Bottom: (002) peak for all samples. Only the sample gathered after ultrasonic treatment showed a shift to higher angles. The symbol * identifies a $\mathrm{WO}_{3}$ peak.
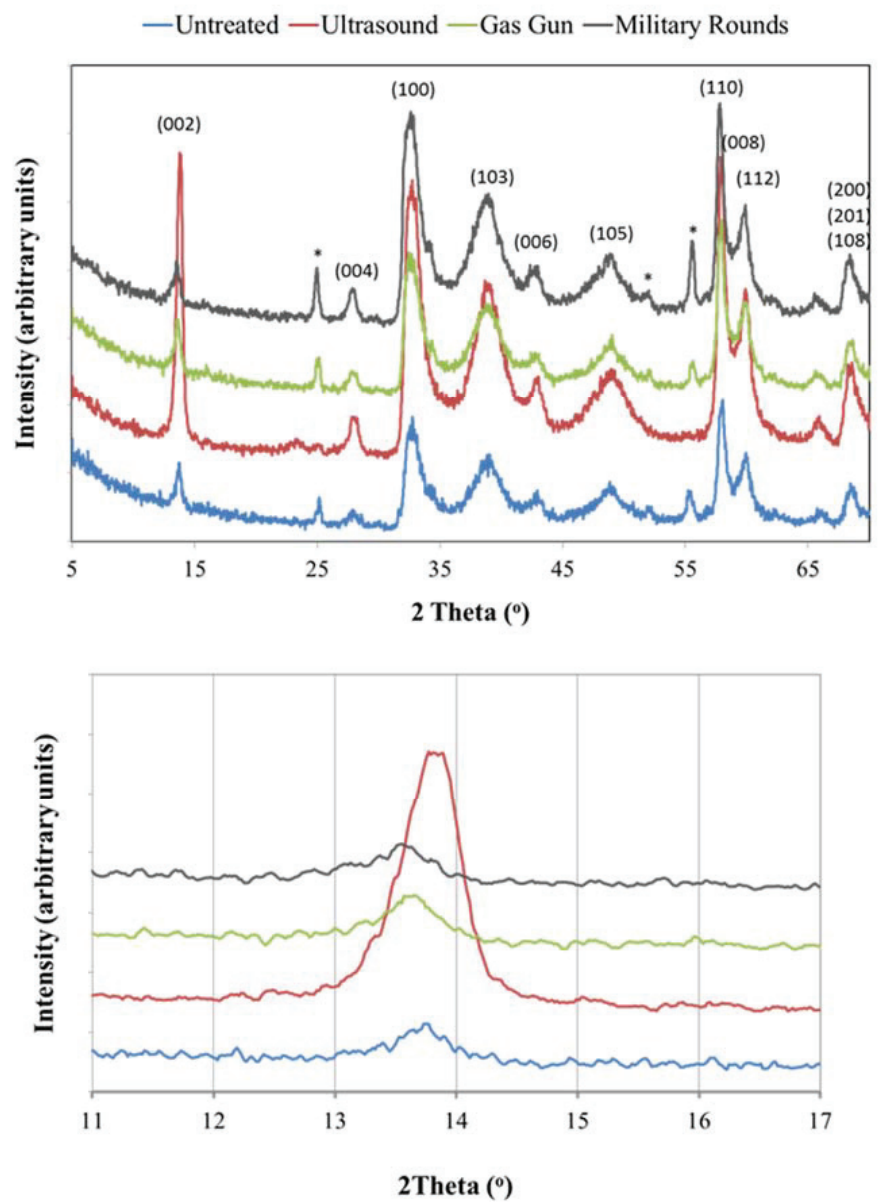

Given those observations, we have denominated this transformation or fracture mode as "agglomeration", since the collapsed particles seem to have larger dimensions after the experiment, implying the agglomeration of many of the original IF spheroids. However, agglomeration is a general term that only refers to the enlargement of the particles and does not completely account for all the complex steps that the particles suffer during this failure mode. Some of the stages we believe are necessary for the 2D polymorphs to acquire the sizes observed imply: (i) an initial crack formed in the surface of the IF particles; (ii) crack propagation towards the core, leading to a complete fracture of the walls; (iii) a structure collapse and disappearance of the hollow cores; (iv) sheets re-arrangement; and (v) new bonds being formed. Moreover, the large sheet-like particles in Figure 3c show little evidence of grain boundaries, which suggests the involvement of a sintering or crystal growth 
mechanism, rather than simple agglomeration. Some of the flattened sections (Figure 3c) reached even micrometer lengths, far from the $143 \mathrm{~nm}$ average diameter in the untreated IF-WS2.

Figure 3. SEM micrographs of (a) untreated particles, (b) samples treated with ultrasonic horn during $3 \mathrm{~h}$ at $20 \%$ amplitude (17 $\mu \mathrm{pp}$ ). Note the appearance of layered particles of much larger dimensions than original spheroids; (c) Particle diameter comparison between the two samples.

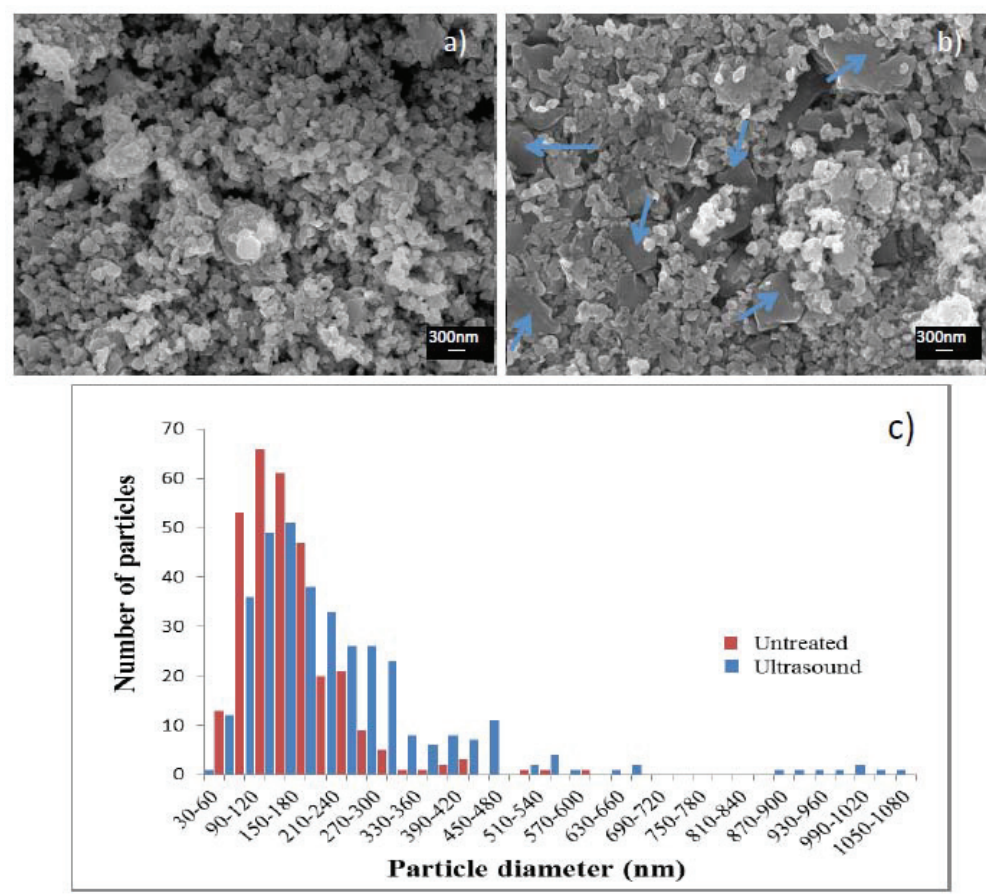

The temperature during the sonication experiments was controlled by an ice bath to prevent solvent evaporation. Nevertheless, given that the particles were exposed to the ultrasonic waves during extended periods of time (up to 10,800 s in some cases) the process seems to have provided enough energy to allow all the steps mentioned above to happen (i-v), followed by crystal growth. The fact that the (002) peak for ultrasound sample, in Figure 2, is much more intense than the one from the untreated specimen supports the idea that more particles with such orientation exist, as is typical of layered structures, fact now verified also by the SEM data.

The study of the ultrasonic sample postmortem by transmission electron microscopy corroborates the existence of both the 2D and 3D (IF) polymorphs. The TEM study of the IF particles that did not collapse presented evidence of crack initiation in the vertices of the polyhedrons. Figure 4 (left) shows one of those cracks, which has not yet propagated through the whole structure. In a similar fashion that surface defects can promote the fracture of macroscopic objects at smaller levels of stress than the ones predicted by theoretical calculations, in IF-WS 2 nanostructures the imperfections in the 


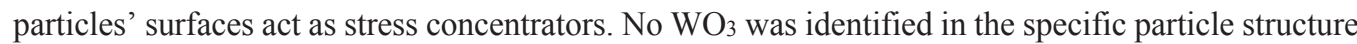
where such observations were made, ruling out its effect in the particle fracture mechanisms.

Figure 4. Left: TEM micrograph of a particle treated with ultrasonic horn that presents a surface crack. The inset is a magnified version of the area pointed by the arrow; Right: TEM studies corroborated the damage of both, internal and external layers of the IF structures.
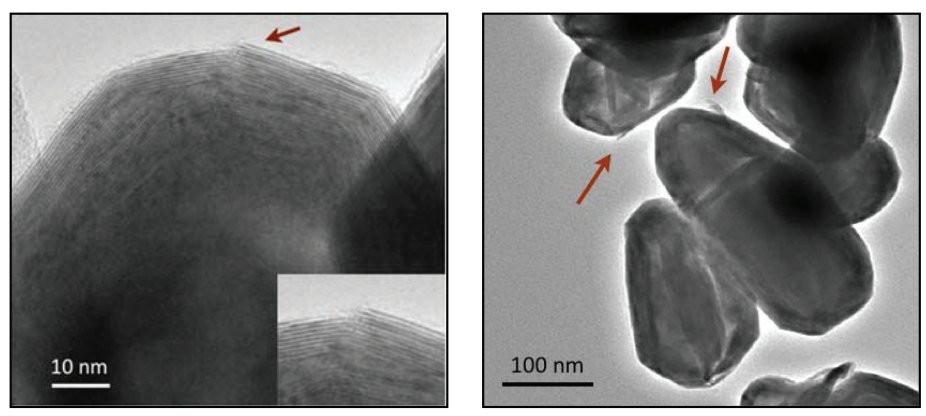

As pointed out in multiple references [19-22], materials do not fracture while in compression, they fracture in tension. The failure modes observed in samples post ultrasonic treatment present a similar signature as the ones expected from fatigue experiments; they resemble the fracture of materials that have been subjected to a cyclic load which involves the contraction and expansion of the structure. We found evidence of breakage in the surface (crack in Figure 4 left) presumably created during one of the expansion cycles while in tension. However, there is also evidence of some of the sheets breaking inside the particles' core (Figure 4 right), indicating a similar mechanism occurring but in the opposite direction, pointing to a cyclic load.

The complete breakage of the outer layers in the crack area (in Figure 4 left) seem to happen without extensive plastic deformation, resembling the typical brittle failure. Such feature is observed in many particles and diverse sections of the sample. However, we also identified particles that present a slightly larger separation between the first and second layers of the lattice than the one found for the inner layers. Such a fact can be interpreted as evidence of a very small amount of plastic deformation occurring in the direction perpendicular to the lattice fringes.

Apart from the multiple particles suffering the agglomeration type failure (fracture-agglomerationphase transformation steps described before), a second mechanism was identified by the observation of the samples by TEM: incipient "delamination". Indeed, small sections ( $c a .10-15 \mathrm{~nm})$ of a few of the IF particles seem to partially separate from the main body of the structure. Figure 4 (right) exemplifies that case, the arrows pointing to sites of partial delamination. A similar exfoliating mechanism has been observed by other groups $[17,23]$. We did not find evidence of larger sections or complete layers totally separating from the IF spheroids in the ultrasonic treated specimens. The amount of particles presenting partial delamination, when compared to the amount that presented agglomeration, was minimal.

The main difference found between the two failure modes is that agglomeration mechanism destroys the hollow cage structures, generating 2D extended structures, and delamination does not. The main 
parallelism between them is that both, incipient delamination in the outer layers of the IF structures, and crack formation with propagation towards the particle core are observed in the vicinity of vertices, edges or surface defects.

The fact that the vertices in untreated particulates presented a larger average interlayer distance $(0.63 \mathrm{~nm})$ than the faceted sides or walls $(0.62 \mathrm{~nm})$ is suspected to have an influence over which failure more (agglomeration $v$ s. delamination) is predominant. However, the difference between such values is so small that more data and a higher resolution instrument might be required to be able to make a definitive correlation.

There is no evidence that suggest that one failure mode represents a first step towards the other. The transformation between the IF particles and the 2D structures in the agglomeration mode seem to start with a crack that advances towards the particle core, feature observed in samples treated in different conditions. The few cases of delamination seen do not exhibit such crack directionality but a separation of only the outer layers. The failure modes do not seem to be associated to particle sizes either. Perfect spherical particles might present a correlation between such since the size will determine the radius of curvature, which is a factor that affects the crack propagation in macroscopic objects.

One of the main limitations we encountered during this study was the lack of a method to quantify the level of damage that the samples suffered. From the SEM images we could estimate the percentage of the sample surface that transformed into larger particles due to the agglomeration mechanism (as in Figure 3). However, multiple images need to be taken to assure that the features observed actually represent the bulk of the sample and the existence of the flat particles underneath the surface layer of the sample could be overlooked. Since the most common failure mechanism observed by large was agglomeration, and only very small sections of the sample delaminated, we contemplated the use of surface area measurements to evaluate the level of damage. Indeed, most particles suffer an enlargement that will produce a surface area reduction (due to the agglomeration mechanism) and only a few of them will have more area exposed (due to delamination).

The surface area data from samples treated with the ultrasonic horn at diverse amplitudes was calculated using the BET methodology and is presented in Figure 5. Samples treated at higher amplitudes or for longer periods of time (not shown) suffer an evident surface area reduction. Both amplitude and time imply more energy being imparted to the particles. The former because the energy transported by a wave is directly proportional to the square of the amplitude of the wave: A high-energy wave is characterized by a high amplitude; a low-energy wave is characterized by a low amplitude. The latter, because energy is calculated directly from the product of the Watts provided by the source and the time of the treatment. The trend towards smaller surface areas in Figure 5 could be correlated to particle agglomeration and the presence of extended 2D layered structures, as the ones seen in the example presented in Figure 3.

The BET surface area measurement does not provide a definitive way to evaluate damage when both agglomeration and delamination occur in large extents. However, it provided a way to compare the extent of damage in bulk samples when agglomeration was the dominant mechanism (as in the ultrasound treated samples) without the need for lengthy SEM/TEM analysis. 
Figure 5. Surface area analysis $\left(\mathrm{m}^{2} / \mathrm{g}\right) v s$. amplitude found for samples subjected to ultrasonic treatments.

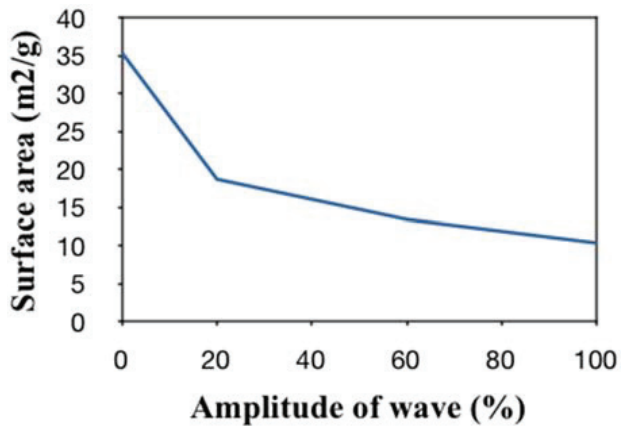

As previously stated, the study of samples by electron microscopy provided a more definitive proof of the changes that the IF-WS2 structure suffered under load than the shifts observed in the XRD analysis alone. Such was particularly true for the samples subjected to shockwave tests and impacted with military rounds, since smaller amounts of powder could be analyzed and only particulates from insulted regions were collected. See Figure 6.

Figure 6. Cracks in the vertices of the particles are the only evidence of damage in samples subjected to shock tube tests. No crack propagation or structure collapse was observed. No evidence of delamination was found either.

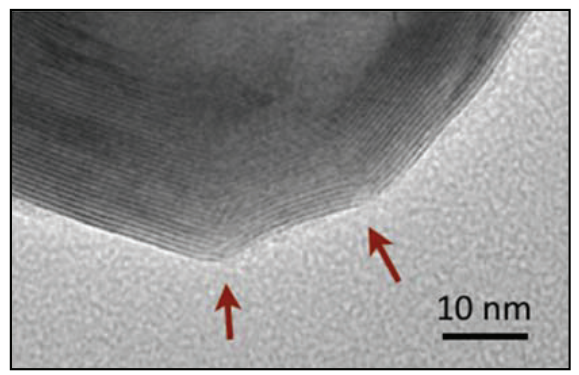

The evaluation of the samples treated by shockwaves revealed that the damage sustained was less than the one observed for ultrasonic methods, as expected from the low pressures registered by the sensors. The SEM observation of the sample from shock tube revealed that only very small sections of the sample showed agglomeration. TEM images showed crack initiation and Figure 6 exemplifies this feature. The arrows on the image point to two different sites where the crystal structure fractured. The pressures used by shockwaves produced by $\mathrm{N}_{2}$ and by He were not enough to cause the sample to suffer a complete collapse. We did not find evidence of delamination in any of the multiple particles analyzed. Thus, we found no evidence that the agglomeration failure mode is preceded by delamination.

Samples impinged with military rounds suffered a larger level of damage than that observed for shock tube tests. Unfortunately, as stated before, it was difficult to collect large amounts of sample in the circumference left by the penetrator, reason for the XRD and BET data not to be as reliable as the 
one seen for ultrasonic treatments. Nevertheless, the SEM analysis of the sample provided proof of the same mechanisms described earlier in this article. We found layered structures similar to the ones in Figure 3b; however, in this case the agglomerates reached not only micrometer dimensions in length and width, but also in height. There are regions in the postmortem sample that the IF particles can still be encountered but the agglomerates are much more abundant.

Figure 7 shows both, the more frequently observed agglomeration (left) and an image of the less common delamination (right). Regarding the agglomeration case, once the IF particles fracture at the polyhedron vertices or edges, they collapse, their sheets re-arrange to diminish any spaces in between layers and are added to the larger crystals in a fashion similar to the one observed during crystal growth (Figure 7 center). In Figure 7 left, grain boundaries mark the regions where IF particles have joined the main particle. The letters in the figure represent: (a) matter has completely been incorporated onto the agglomerate and is now part of the surface to which new particles will connect; (b) the grain boundaries of the initial IF particle are still visible within the main body of the agglomerate; (c) a new layer is being formed, some sections of the same present layered structure with sharp edges and others partially conserve the spherical shape; and (d) an IF-WS2 semispherical particle still lays in the surface.

Figure 7. Sample recovered from impact with military rounds. Note the similarities between regions where IF have been added to an existent layered structure, such as $a, b$, c, and d (left) with sites proposed by crystal growth theories for addition matter in a surface (center). Particle showing layers that delaminated (right).

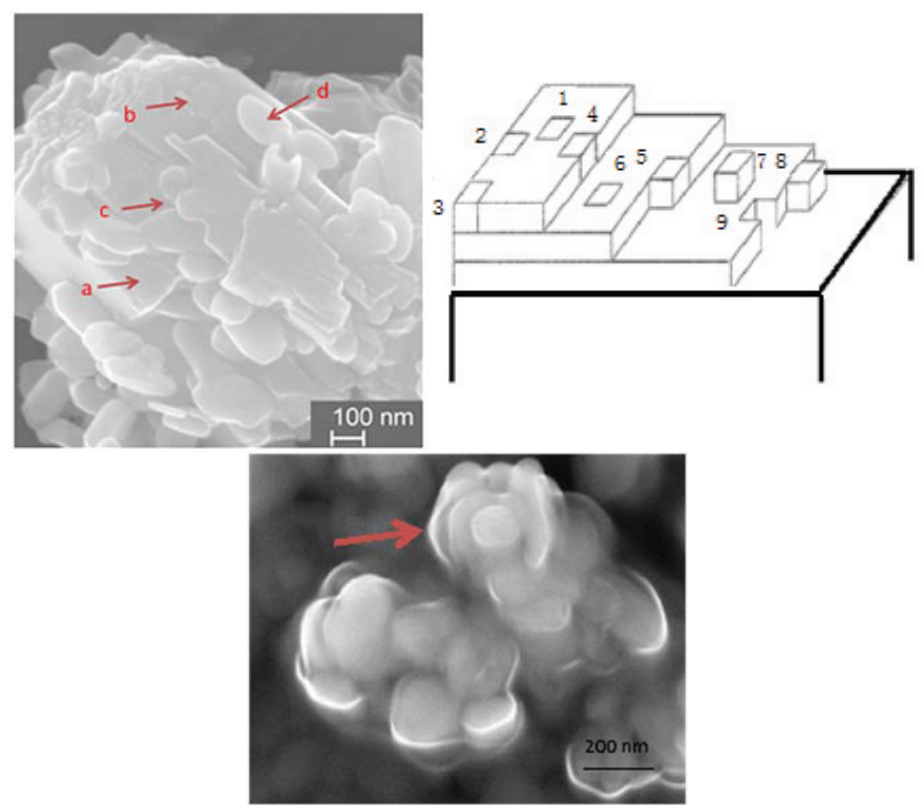

The angles in the vertices in the polyhedral particles seem to play a similar role than the radius of curvature of the surface or internal cracks have in traditional fracture mechanics analysis. Furthermore, the larger crystal to which other IF are being added present imperfections such as kinks, 
ledges, steps, etc. Each of those sites represent regions in the sample where dangling bonds exist; those locations are more prone to accept the addition of new matter to reduce the overall surface energy as is usually described by crystal growth theories [24,25]. According to the latter, the thermodynamics of crystal surface formation and transformation is based on the idea that the energy that each atom's position on a crystal surface is determined by the number of neighboring atoms and the amount of unsatisfied bonds. The numbers in Figure 7 (center) represent: surface atoms, surface vacancies, adatoms, kinks, and steps. Figure 7 (right): this image represents the only instance that we found of what it seems as a complete layer delamination; the outer sheets of the material seem to come apart to reveal an inner particle still intact.

The effect of temperature in the material agglomeration during the military round impact certainly plays a significant role to transform the IF into extended sheet-like $\mathrm{WS}_{2}$ structures. However, given the complexity of the test, no temperature values were recorded and the heat effect will only be subject of further study and discussion in future reports.

Figure 8 summarizes our findings. In the agglomeration mechanism: IF particles are more susceptible to breakage in areas where defects or discontinuities, such as edges and vertices, are located. Those act as stress raisers and are the primary sites where crack initiates, in the outer layers of the cage structures. Once the crack propagates it will reach the hollow cores, which will promote the particles collapse and the transition between the 3D and the $2 \mathrm{D} \mathrm{WS}_{2}$ polymorphs. The layered structures will agglomerate to reach micron scale dimensions. The collapse and agglomeration of the IF structures was the main effect identified as a consequence of the application of ultrasonic treatment in diverse conditions and the use of military rounds despite the diverse time scale of each of those methods. Shock waves in the conditions employed only produced surface cracks without the full structure collapse. Agglomeration signature is identified as the predominant mechanism in the experimental conditions used.

Figure 8. Representation of the two failure modes identified in the IF- $\mathrm{WS}_{2}$ structures.

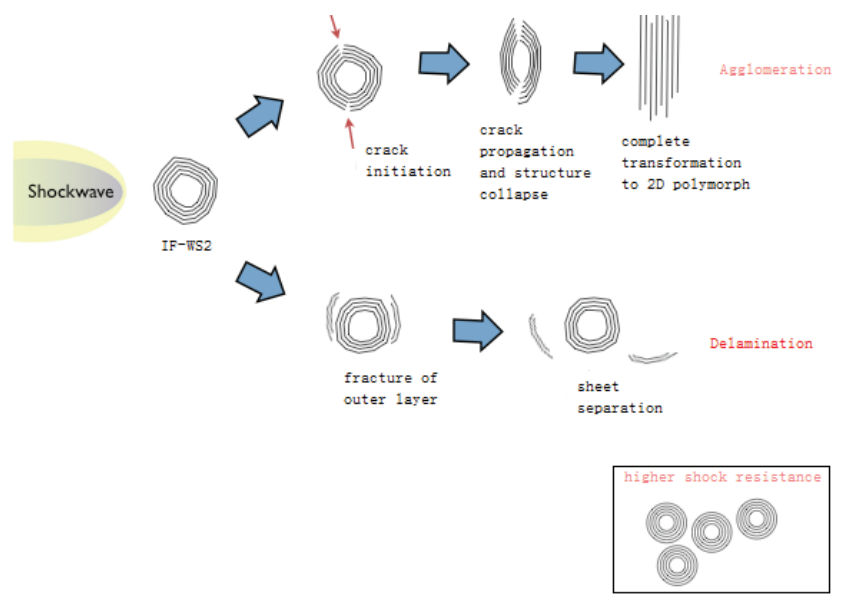


We also identified evidence that suggests that the particles can suffer delamination. The areas in the IF particles surface where this failure mode was identified also correspond to locations where defects or discontinuities are observed. During this failure mode the Van der Waals attraction keeping the layers in the together is broken as a consequence of the shock and small portions of the outer layer come apart, leaving behind sections with a smaller diameter particle but still IF characteristics. The separation between surface layers do not seem to propagate into internal layers. Remarkably, during our experiments we observed incipient delamination in only very few particles treated by sonication. No correlation was found between the extent of this failure mode and times of treatment. We observed only one instance of an almost complete layer separation (Figure 7c).

In sum, all the electron microscopy observations conducted in the postmortem products point to the existence of surface flaws as starting point where agglomeration or delamination take place, independent of the amount energy imparted by the insult, the time of the event or the isotropic or non-isotropic nature of the experiment.

The structure fails by two modes: either as a consequence of the creation of a surface crack and its propagation into the hollow core, followed by the structure collapse and resulting in the transformation into a 2D sheet-like polymorph and the agglomeration of particles into larger bodies, or by delamination of small sections of the sample, which does not completely destroy the IF structure but removes superficial sections, leaving the hollow cores and inner walls intact.

What conditions favor agglomeration over delamination is still subject of study. No link has been found here between the two fracture mechanisms and levels of stress. Crack formation, usually followed by propagation and structure collapse has been identified without evidence of delamination at diverse levels of stress and over a wide range of times of treatment. The interlayer separation and the radius of curvature (given by particle size and existence of faceted structures) are suspected to play a role on which mode dominates however, such has not been clearly demonstrated/quantified.

Studies of brittle fracture demonstrate that the experimental fracture strengths of most materials are lower than the theoretical ones (based on calculations related to atomic bond energies) due to the existence of microscopic flaws that act as stress raisers, amplifying the stress at a given point. The postmortem TEM analyses indicate that the IF-WS 2 particles deform and break where structural discontinuities appear: defects and edges in the polyhedral shapes, along the degree of curvature of the particulates, seem to play a critical role on the material resistance to breakage, following a pattern similar to the ones observed in more general fracture mechanics principles. These findings provide evidence that without the stress concentrators in the particle surface, as would be the case if more spherical particles were used, the material should be able to support higher pressure loadings, supporting previous reports [17]. The bottom of Figure 8 shows a schematic representation of how such particles will look like.

No evidence of crack formation or delamination was seen in the more uniform sections of the particle surfaces. These results suggest that IF-WS 2 is prone to show a phase transformation into a $2 \mathrm{D}$ polymorph and only near spherical particles might show remarkable shock absorption characteristics. Despite finding that the structures have similar failure modes than their carbon counterparts, which can diminish their shock resistance, our findings also point to a solution to the failure: use of 
defect free (more spherical, not faceted) particulates, supporting previous statements made by Tenne et al. [17].

It is worth noting that the creation of defect free IF-WS 2 particles might prove challenging; the use of completely spherical $\mathrm{WO}_{3}$ produced by microwave plasma methods in our labs and reacted with $\mathrm{H}_{2} \mathrm{~S}$, produced highly faceted IF-WS 2 , see Figure 9. Different treatment conditions should be developed in order to achieve completely spherical nanoparticles.

Figure 9. IF-WS 2 generated at $900{ }^{\circ} \mathrm{C}$ from the $\mathrm{WO}_{3}$ produced using a microwave atmospheric plasma system. Despite the highly spherical nature of the precursor, the IF-WS 2 particulates present facets and polyhedral shapes. A few atoms thick $2 \mathrm{D} \mathrm{WS}_{2}$ were also observed.

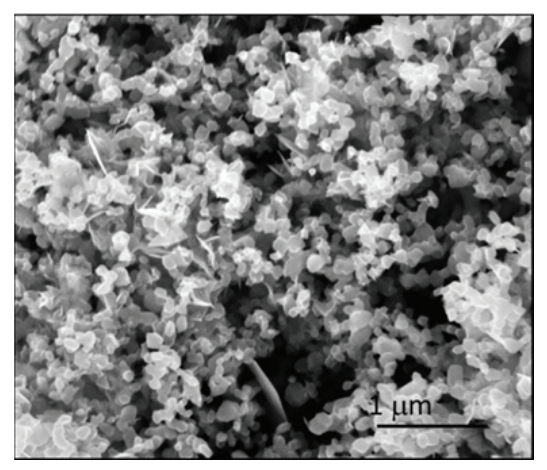

With failure modes now clearly characterized future research could (a) correlate the cracks observed, the angles at the vertices and the levels of stress used, to generate mathematical expressions that could predict mechanical properties such as fracture toughness for the particular size of IF-WS2 and (b) design nanoparticles against fracture.

\section{Experimental Section}

Commercially manufactured inorganic fullerene-type tungsten disulfide (IF-WS 2 ) was obtained in the form of a commercial lubricant Nanolub (NanoMaterials Ltd., Ness Ziona, Israel). The sample was washed with ethanol to remove additives; the solvent was separated using an Eppendorf (New York, NY, USA) Model 5418 centrifuge and the IF-WS2 particles dried at room temperature in a desiccator. The sample was studied by XRD and SEM before being subject to treatments and as postmortem.

\subsection{Methods Used to Induce Failure}

The ultrasonic tester used for this procedure was a Sonomechanics (New York, NY, USA) $1200 \mathrm{~W}$ ultrasonic liquid processor shown in Figure 10. The equipment consists of a generator that provides electric signal input to the piezoelectric transducer, a water-cooled barbell horn and a protective enclosure. The samples were placed in a solvent (water or ethanol) and the beaker containing such dispersion was placed in a second beaker, which was filled with iced water and a copper coil that was 
also circulating cold water to maintain the bath at a constant temperature. The generator produced a constant frequency of $20 \mathrm{kHz}$ at the horn tip; however diverse samples were exposed to the ultrasonic waves during different conditions: the amplitude was varied between $20 \%$ and $100 \%$ (which correspond to 17 to $81 \mu \mathrm{pp}$, respectively) and times of exposure extended from 3600 to $10,800 \mathrm{~s}$.

Figure 10. Ultrasonic horn setup. Note that a cooling bath was used to maintain a constant temperature, however the bath that included the cooling coil and the sample in the liquid media were not in contact. The latter were placed in a second beaker. The blue foam was used to keep the inner beaker in place.

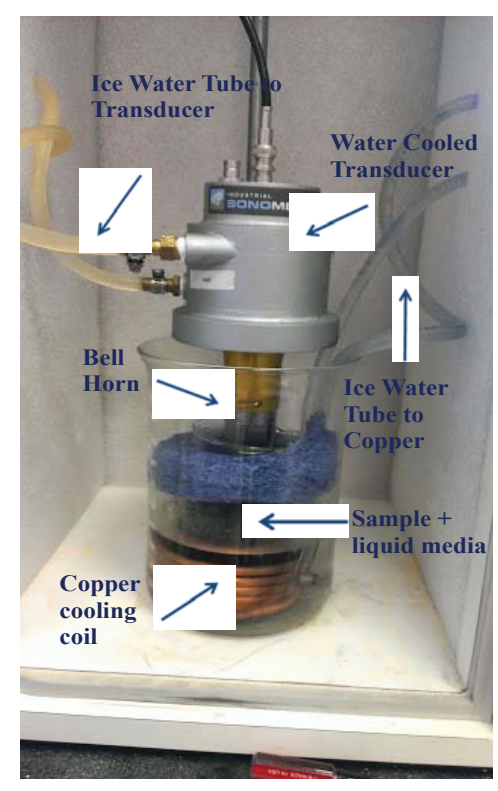

A shock tube was used for creating a shock wave that propagated and impinged on the sample. A Kevlar layer was positioned on the surface of the particles to prevent their dispersion inside the shock tube. The system uses a pressurized driver section and evacuated driven section separated by a pair of diaphragms to create a supersonic wave that propagates along the tube and impinges on a test holder at the end of the device. Pressure transducers are located along the tube allow for monitoring of the shock and measuring its speed and strength (pressure sensors 1, 2, and 3 in Figure 11). Detailed theory can be found in [26].

The material to be tested was attached to the holder and bolted onto the end section where pressure transducer cable was connected. The shock tube was then loaded with two heat-treated copper diaphragms 0.025 inches thick scored to a depth of 0.013 inches. Vacuum was then drawn to an absolute pressure of approximately $2 \mathrm{~mm} \mathrm{Hg}$ in the driven section through the use of a roughing pump. When this was complete, gas was added in a controlled manner until the driven section reached $25 \mathrm{~mm} \mathrm{Hg}$. The vacuum gauge was isolated and then disconnected to prevent damage to it during creation of the pressure event. The driver section was then loaded with either Nitrogen or Helium (depending on the desired Mach number) to $720 \mathrm{psig}$. When the pressure gauge reached $350 \mathrm{psig}$, the 
mid-section was isolated. The shock tube was then fired by opening the firing valve. As pressurized gas entered the diaphragm section the pressure differential across the second diaphragm caused it to burst creating a wave through the driven section. The subsequent drop in pressure in the diaphragm section caused the first diaphragm to burst and the formation of a second, faster wave. The second wave overtook the first and the two coalesced into a single wave that traveled along the driven section and to the sample holder.

Figure 11. Shock tube setup (top) and image of the sample contained with Kevlar (bottom).

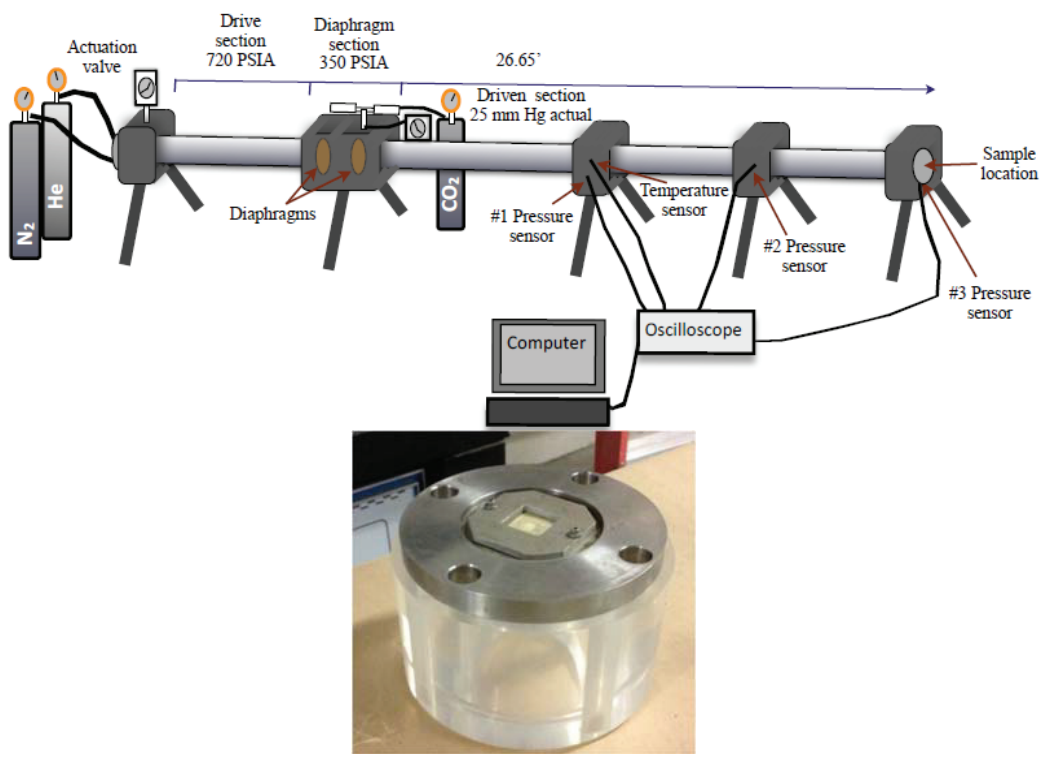

For the impact with military rounds the IF powder sample was dispersed in between Kevlar layers and the layers introduced into a nylon pouch. The later was then positioned in front of a clay bed and a $7.62 \mathrm{~mm}$ NATO round was then fired at it as indicated in Figure 12. The IF-WS 2 particles located close to the hole left by the penetrator were then collected and analyzed by the methods described in the next section.

Figure 12. Sample testing setup (left) and orifice left by penetrator in a nylon bag that contained the sample in between Kevlar layers (right).
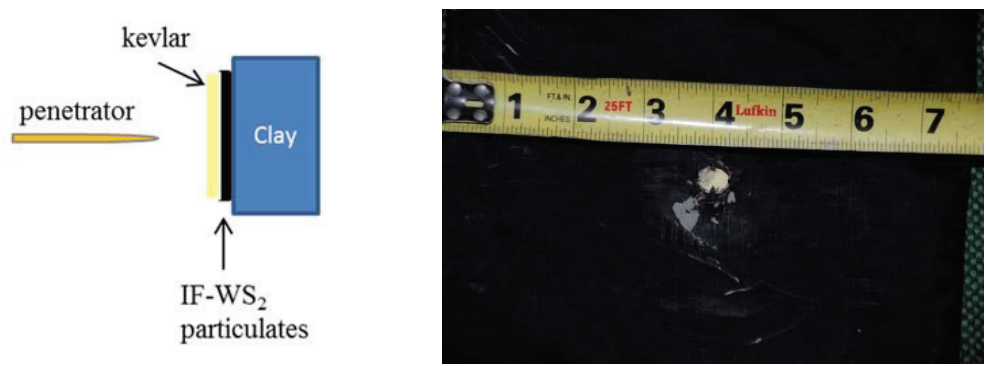


\subsection{Methods Used to Characterize Samples}

A Philips 1830 PAnalytical X-Ray Diffractometer (Almelo, The Netherlands), using a copper source, $\mathrm{K} \alpha$ of $1.54 \AA$ was employed for the analysis of the crystalline components of the samples. The powders were positioned into a silicon zero background sample holder and the diffraction patterns recorded between $5^{\circ}$ and $70^{\circ}(2 \theta)$ with $0.020^{\circ}$ step size and one second per step.

A JEOL (Tokyo, Japan) 2010F FASTEM field emission gun scanning transmission electron microscope (STEM/TEM) equipped with Gatan (Pleasanton, $C A$, USA) GIF image filtering system was used to collect images and electron diffraction patterns. The samples were dispersed in ethanol and a drop of the mixture was allowed to dry in a Holey carbon copper grid. The JEOL 2010F lattice resolution is $0.1 \mathrm{~nm}$.

The microstructural features of the specimens post treatment were analyzed using a Zeiss Neon (Oberkochen, Germany) 40 High Resolution Scanning Electron Microscope (SEM). Images were acquired at diverse magnifications while the microscope was operated between 5 and $20 \mathrm{kV}$. Energy Dispersive Spectroscopy (EDS) experiments were conducted in conjunction with the SEM using an Apollo 10 silicon drift detector (SDD). Data was collected and analyzed using Genesis Spectrum software (EDAX, Mahwah, NJ, USA).

Brunauer Emmet Teller (BET) surface area analysis was performed employing a Quantachrome (Boynton Beach, FL, USA) Nova 4200 Physisorption analyzer. A degas step was conducted prior to the analysis. The measurements were done using nitrogen atmosphere.

\subsection{Attempts to Produce Highly Spherical IF-WS 2}

Highly spherical $\mathrm{WO}_{3}$ was generated in an atmospheric plasma torch microwave system following procedures previously reported $[27,28]$. The $\mathrm{WO}_{3}$ sample was then transformed into IF-WS 2 by a thermal treatment in a tubular furnace operated at $900{ }^{\circ} \mathrm{C}$ for a period of $2 \mathrm{~h}$ using $\mathrm{H}_{2} \mathrm{~S}$ atmosphere.

\section{Conclusions}

The microstructural changes observed in the IF-WS 2 particulates as a consequence of treatments with ultrasonic horn, shock tube and military rounds could be categorized in two distinct fracture modes.

The most commonly observed was the phase transformation of the $3 \mathrm{D}$ cage-like structures to the 2D layered polymorphs, which involved a crack forming at the particulate surface leading to the structure collapse and subsequent agglomeration of the plate-like sheets, to produce larger particle sizes. Such mechanism dominated the samples' microstructure for experiments performed using ultrasonic waves and the ones exposed to military rounds. Incipient cracks were identified in shock wave postmortem samples.

A less common secondary mechanism of particle breakage was also identified; the delamination of IF-WS 2 . However, the later was mainly observed as an incipient process, with only very small sections of the shells separating from the IF main body and the survival of the IF hollow cage characteristics.

The steps required for particle failure by the first mechanism included: (i) an initial crack formed in the surface of the IF particles; (ii) crack propagation towards the core, leading to a complete fracture of the walls; (iii) structure collapse and disappearance of the hollow cores; (iv) sheet re-arrangement; 
and (v) new bonds being formed, which resulted in an overall agglomeration. We encountered evidence that the IF-WS 2 structure collapse initiated at the edges of the polyhedral particles, which acted as stress concentrators, demonstrating that general fracture mechanics concepts can be applied to materials at the nanoscale. Based on those findings and in agreement with previous reports, defect-free perfectly spherical IF-WS 2 surfaces are expected to present improved shock absorbing performance than that observed for polyhedral shape IFs.

We found that surface area measurements could be correlated to the extent of particle damage when agglomeration could be recognized as the main effect of the pressure load.

We demonstrated that more irregular particles (faceted) tend to fail at defect sites that act as stress concentrators independently of how energy is delivered: shock being applied in fractions of a second, or over long periods of time, as an isotropic or non-isotropic event, as a single occurrence or by cyclic treatment.

\section{Supplementary Information}

Figure S1. TEM image of IF-WS 2 nanoparticles (left) along EDS spectra (right) showing that in some locations $\mathrm{WO}_{3}$ can be identified.
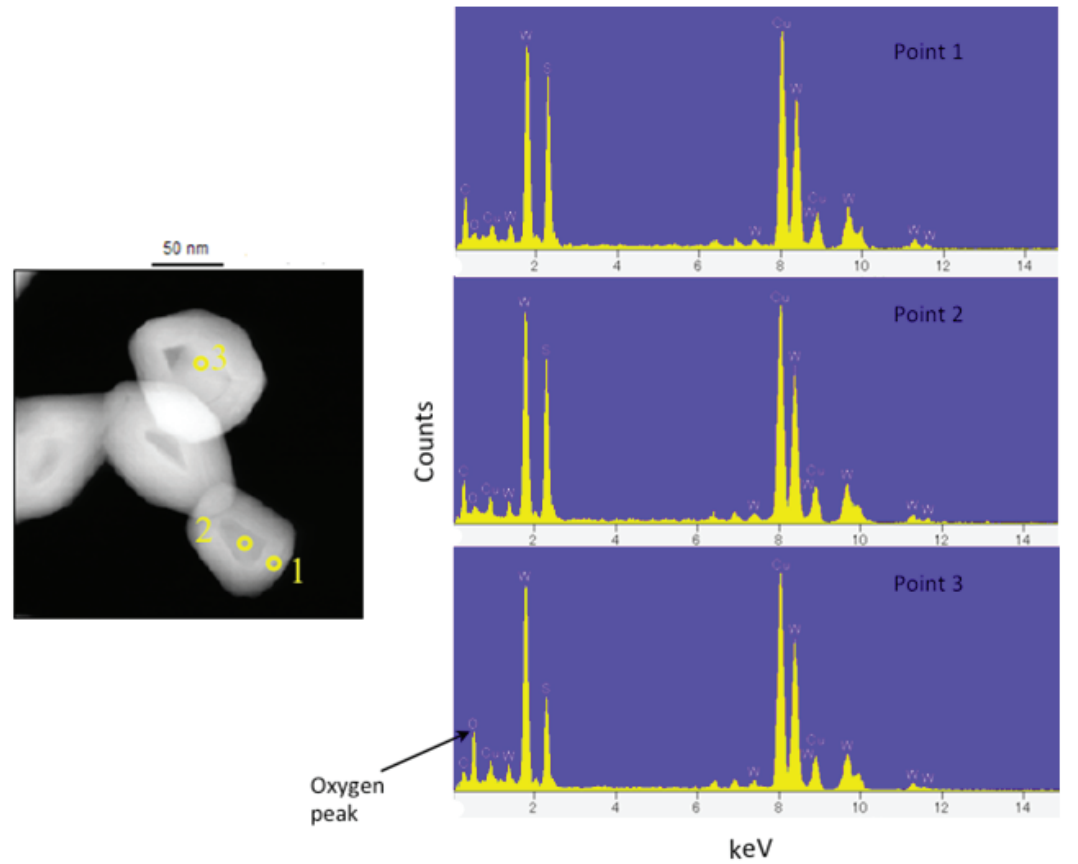

\section{Acknowledgments}

This work was conducted with support of the Office of Naval research, Code 30. We appreciate the help that Chris Clay and John Gibson provided to conduct the shock tube tests. We thank Jing Bing from University of New Mexico and JEOL, USA Inc. for facilitating the collection of TEM data. We also thank Alexey Peshkovsky, from Industrial Sonomechanics for guidance using the transducer. 


\section{Author Contributions}

The findings in this manuscript are part of Jamie Cook's Master degree thesis work. Claudia C. Luhrs advised the thesis and directed the research. Garth Hobson guided the shock tube tests. Lou Roncase conducted the military round impacts and Steven Rhyans performed a summer internship aiding the team with some of the ultrasonic experiments.

\section{Conflicts of Interest}

The authors declare no conflict of interest.

\section{References}

1. Tenne, R.; Margulis, L.; Genut, M.; Hodes, G. Polyhedral and cylindrical structures of tungsten disulfide. Nature 1992, 360, 444-446.

2. Margulis, L.; Tenne, R.; Iijima, S. Nucleation of $\mathrm{WS}_{2}$ fullerenes at room temperature. Microsc. Microanal. Microstruct. 1996, 7, 87-89.

3. Feldman, Y.; Frey, G.; Homyonfer, M.; Lyakhovitskaya, V.; Margulis, L.; Cohen, H.; Hodes, G.; Hutchison, J.; Tenne, R. Bulk synthesis of inorganic fullerene-like $\mathrm{MS}_{2}(\mathrm{M}=\mathrm{Mo}, \mathrm{W})$ from the respective trioxides and the reaction mechanism. J. Am. Chem. Soc. 1996, 118, 5362-5367.

4. Rapoport, L.; Bilik, Y.; Feldman, Y.; Homyonfer, M.; Cohen, S.; Tenne, R. Hollow nanoparticles of $\mathrm{WS}_{2}$ as potential solid-state lubricants. Nature 1997, 387, 791-793.

5. Tenne, R.; Homyonfer, M.; Feldman, Y. Nanoparticles of layered compounds with hollow cage structures (inorganic fullerene-like structures). Chem. Mater. 1998, 10, 3225-3238.

6. Kroto, H.; Heath, J.; Obrien, S.; Curl, R.; Smalley, R. C-60-Buckminsterfullerene. Nature 1985, 318, 162-163.

7. Kroto, H. The stability of the fullerenes C-24, C-28, C-32, C-36, C-50, C-60 and C-70. Nature 1987, 329, 529-531.

8. Vul', A.; Davidenko, V.; Kidalov, S.; Ordan'yan, S.; Yashin, V. Fullerenes catalyze the graphite-diamond phase transition. Tech. Phys. Lett. 2001, 27, 384-386.

9. Rapoport, L.; Fleischer, N.; Tenne, R. Applications of $\mathrm{WS}_{2}\left(\mathrm{MoS}_{2}\right)$ inorganic nanotubes and fullerene-like nanoparticles for solid lubrication and for structural nanocomposites. J. Mater. Chem. 2005, 15, 1782-1788.

10. Diez-Pascual, A.M.; Naffakh, M. Mechanical and thermal behaviour of isotactic polypropylene reinforced with inorganic fullerene-like $\mathrm{WS}_{2}$ nanoparticles: Effect of filler loading and temperature. Mater. Chem. Phys. 2013, 141, 979-989.

11. Naffakh, M.; Diez-Pascual, A.M.; Marco, C.; Ellis, G.J.; Gomez-Fatou, M.A. Opportunities and challenges in the use of inorganic fullerene-like nanoparticles to produce advanced polymer nanocomposites. Prog. Polym. Sci. 2013, 38, 1163-1231.

12. Diez-Pascual, A.M.; Naffakh, M.; Marco, C.; Ellis, G. Rheological and tribological properties of carbon nanotube/thermoplastic nanocomposites incorporating inorganic fullerene-like $\mathrm{WS}_{2}$ nanoparticles. J. Phys. Chem. B 2012, 116, 7959-7969. 
13. Tehrani, M.; Luhrs, C.C.; Al-Haik, M.S.; Trevino, J.; Zea, H. Synthesis of $\mathrm{WS}_{2}$ nanostructures from the reaction of $\mathrm{WO}_{3}$ with $\mathrm{CS}_{2}$ and mechanical characterization of $\mathrm{WS}_{2}$ nanotube composites. Nanotechnology 2011, 22, 285714.

14. Zohar, E.; Baruch, S.; Shneider, M.; Dodiuk, H.; Kenig, S.; Tenne, R.; Wagner, H.D. The Effect of $\mathrm{WS}_{2}$ Nanotubes on the Properties of Epoxy-Based Nanocomposites. J. Adhes. Sci. Technol. 2011, 25, 1603-1617.

15. Naffakh, M.; Diez-Pascual, A.M.; Marco, C.; Gomez, M.A.; Jimenez, I. Novel melt-processable poly(ether ether ketone)(PEEK)/inorganic fullerene-like $\mathrm{WS}_{2}$ nanoparticles for critical applications. J. Phys. Chem. B 2010, 114, 11444-11453.

16. Zhu, Y.; Sekine, T.; Li, Y.; Wang, W.; Fay, M.; Edwards, H.; Brown, P.; Fleischer, N.; Tenne, R. $\mathrm{WS}_{2}$ and $\mathrm{MoS}_{2}$ inorganic fullerenes-Super shock absorbers at very high pressures. Adv. Mater. 2005, 17, 1500-1503.

17. Zhu, Y.; Sekine, T.; Li, Y.; Fay, M.; Zhao, Y.; Poa, C.; Wang, W.; Roe, M.; Brown, P.; Fleischer, N. Shock-absorbing and failure mechanisms of $\mathrm{WS}_{2}$ and $\mathrm{MoS}_{2}$ nanoparticles with fullerene-like structures under shock wave pressure. J. Am. Chem. Soc. 2005, 127, 16263-16272.

18. Vasu, K.; Matte, H.S.S.R.; Shirodkar, S.N.; Jayaram, V.; Reddy, K.P.J.; Waghmare, U.V.; Rao, C.N.R. Effect of high-temperature shock-wave compression on few-layer $\mathrm{MoS}_{2}, \mathrm{WS}_{2}$ and MoSe2. Chem. Phys. Lett. 2013, 582, 105-109.

19. Meyers, M.; Chawla, K. Mechanical Behavior of Materials, 2nd ed.; Cambridge University Press: Cambridge, UK, 2009.

20. Griffith, A.A. The Phenomena of Rupture and Flow in Solids. Philos. Trans. Ser. A 1920, 221, 163-198.

21. Irwin, G.R. Fracture Dynamics; American Society for Metals: Cleveland, OH, USA, 1948.

22. Orowan, E. Fracture and Strength of Solids. Rep. Prog. Phys. 1949, 12, doi:10.1088/00344885/12/1/309.

23. Joly-Pottuz, L.; Martin, J.; Dassenoy, F.; Belin, M.; Montagnac, G.; Reynard, B.; Fleischer, N. Pressure-induced exfoliation of inorganic fullerene-like $\mathrm{WS}_{2}$ particles in a Hertzian contact. J. Appl. Phys. 2006, 99, 023524.

24. Kossel, W. Extenoling the law of bravais. Nach. Ges. Wiss. Gottingen. 1927, 143.

25. Stranski, I.N. Zur Theorie des Kristallwachstums. Z. Phys. Chem. 1928, 136, 259-278 (In German).

26. Crinklaw, J.D. Acoustic Shock Testing on Re-entry Vehicle Insulation Material. Master's Thesis, Naval Postgraduate School, Monterey, CA, USA, March 2005.

27. Wakeland, S.; Cui, Y.; Knapp, A.; Richard, M.; Phillips, J.; Luhrs, C. Multilayered nanoparticles generated by plasma methods for energy storage applications. Nanosci. Nanotechnol. Lett. 2012, 4, 316-322.

28. Phillips, J.; Luhrs, C.C.; Richard, M. Review: Engineering particles using the aerosol-through-plasma method. IEEE Trans. Plasma Sci. 2009, 37, 726-739. 


\title{
Nanostructured Boron Nitride: From Molecular Design to Hydrogen Storage Application
}

\author{
Georges Moussa, Chrystelle Salameh, Alina Bruma, Sylvie Malo, Umit B. Demirci, Samuel \\ Bernard and Philippe Miele
}

\begin{abstract}
The spray-pyrolysis of borazine at $1400{ }^{\circ} \mathrm{C}$ under nitrogen generates boron nitride (BN) nanoparticles (NPs). The as-prepared samples form elementary blocks containing slightly agglomerated NPs with sizes ranging from 55 to $120 \mathrm{~nm}$, a Brunauer-Emmett-Teller (BET)-specific surface area of $34.6 \mathrm{~m}^{2} \mathrm{~g}^{-1}$ and a helium density of $1.95 \mathrm{~g} \mathrm{~cm}^{-3}$. They are relatively stable in air below $850{ }^{\circ} \mathrm{C}$ in which only oxidation of the NP surface proceeds, whereas under nitrogen, their lower size affects their high temperature thermal behavior in the temperature range of $1450-2000{ }^{\circ} \mathrm{C}$. Nitrogen heat-treated nanostructures have been carefully analyzed using X-ray diffraction, electron microscopy and energy-dispersive X-ray spectroscopy. The high temperature treatment $\left(2000{ }^{\circ} \mathrm{C}\right)$ gives hollow-cored BN-NPs that are strongly facetted, and after ball-milling, hollow core-mesoporous shell NPs displaying a BET-specific surface area of $200.5 \mathrm{~m}^{2} \cdot \mathrm{g}^{-1}$ and a total pore volume of $0.287 \mathrm{~cm}^{3} \cdot \mathrm{g}^{-1}$ were produced. They have been used as host material to confine, then destabilize ammonia borane $(\mathrm{AB})$, thus improving its dehydrogenation properties. The as-formed $\mathrm{AB} @ \mathrm{BN}$ nanocomposites liberated $\mathrm{H}_{2}$ at $40{ }^{\circ} \mathrm{C}$, and $\mathrm{H}_{2}$ is pure in the temperature range $40-80{ }^{\circ} \mathrm{C}$, leading to a safe and practical hydrogen storage composite material.
\end{abstract}

Reprinted from Inorganics. Cite as: Moussa, G.; Salameh, C.; Bruma, A.; Malo, S.; Demirci, U.B.; Bernard, S.; Miele, P. Nanostructured Boron Nitride: From Molecular Design to Hydrogen Storage Application. Inorganics 2014, 2, 396-409.

\section{Introduction}

Advanced nanostructured materials may be defined as materials having one dimension in the 1 to $100 \mathrm{~nm}$ range. The massive academic and industrial research efforts concerning these materials over the past decade arose from the remarkable variations in their physical and chemical properties when their dimension shrinks to the nanometric scale. In this category of materials, the interest for hexagonal-boron nitride ( $h$-BN, but expressed here as $\mathrm{BN}$ ) grew during the past few decades in relation to their unique combination of key properties.

$\mathrm{BN}$ is a synthetic chemical compound containing boron $(\mathrm{B})$ and nitrogen $(\mathrm{N})$ atoms in a one-to-one ratio. The in-plane atoms are linked through covalent bonds, while the out-of-plane layers are bonded by weak interactions (van der Waals forces) between $\mathrm{B}$ and $\mathrm{N}$ atoms, alternatively, providing anisotropic properties. BN displays a large band gap $(\sim 5.5 \mathrm{eV})$ and offers the lowest density $\left(d=2.26 \mathrm{~g} \cdot \mathrm{cm}^{-3}\right)$ among non-oxide ceramics. It proposes relatively good thermal stability in air and vacuum, high thermal conductivity, good thermal shock resistance, high electrical resistance, a low dielectric constant and loss tangent, microwave transparency, non-toxicity and easy machinability. Furthermore, it is non-abrasive, lubricating and non-reactive towards molten metals [1-6]. 
BN was obtained for the first time by Balmain [7] in 1842 through the reaction between boric oxide and potassium cyanide. It is nowadays produced by conventional powder technology, requiring nitridation or carbothermal reaction of boric acid/boric oxide with melamine or urea and the use of additives during the further sintering process [8]. It is used in various fields of chemistry, metallurgy, high temperature technology, electronics and in thermal management applications. However, beside the fact that the use of boric oxide inherently induces the presence of oxygen-containing phases, BN is only produced as powders with a plate-like morphology and workpieces. This inherently limits the development of $\mathrm{BN}$.

Recently, interest at the academic level has arisen in both the synthesis of nanostructured BN and their applications for energy and the environment [9-13]. The important industrial challenges in line with nanostructured $\mathrm{BN}$ production requires the development of materials in which topologies, shapes and morphologies are tuned on demand. Inherent difficulties of traditional techniques to manufacture such materials can be addressed by the development of synthetic pathways where molecular/inorganic chemistry, processing and material chemistry/science are combined rationally to process $\mathrm{BN}$ with tailor-made properties [14]. The key step in nanostructured BN preparation is the selection of the $\mathrm{BN}$ precursors. Precursors with a good B:N ratio where/for which hydrogen $(\mathrm{H})$ is the only element added to $\mathrm{B}$ and $\mathrm{N}$ are preferred. Borazine and derived polyborazylene are the most appropriate candidates $[15,16]$. Within this context, in this review article, we discuss the use of borazine (BZ) as a single-source molecular precursor used for the design of BN nanoparticles (NPs), hollow-cored BN-NPs that are strongly facetted and hollow core-mesoporous shell NPs. The latter have been used as host materials to encapsulate and store ammonia borane (AB).

\section{Results and Discussion}

\subsection{Borazine-Derived BN Nanoparticles}

Borazine (BZ) had been originally discovered by Alfred Stock in 1926 [17]. It displays a chemical formula $\mathrm{H}_{3} \mathrm{~B}_{3} \mathrm{~N}_{3} \mathrm{H}_{3}$. It is a preformed $\mathrm{B}-\mathrm{N}$-like ring structure and has the correct $\mathrm{B}$-to- $\mathrm{N}$ ratio. Furthermore, it is economically competitive and attractive from a technical point of view, based on its reaction starting from cheap compounds, such as $\left(\mathrm{NH}_{4}\right)_{2} \mathrm{SO}_{4}$ and $\left(\mathrm{NaBH}_{4}\right)$, reacting in tetraglyme at low temperature $\left(120-140^{\circ} \mathrm{C}\right)$ [18]. Borazine offers the advantage of being liquid with an adequate vapor pressure to be applied in gas phase pyrolysis processes to prepare nanostructured $\mathrm{BN}$. As an illustration, we have demonstrated the interest of $\mathrm{BZ}$ to produce $\mathrm{BN}$ nanoparticles by spray-pyrolysis [19-22]. In our process, BZ is nebulized into an aerosol, and the stream consisting of tiny BZ droplets suspended in the carrier gas is transported by the carrier gas to be passed through the preheated tubular furnace at $1400{ }^{\circ} \mathrm{C}$ under nitrogen. In the hot-zone, the conversion of the nebulized precursor occurs through molecular condensation and ring-opening mechanisms involving the evolution of dihydrogen and producing vapors of BN ring-based species. The latter, reacting to form the consolidated boron nitride network, are swept by the nitrogen carrier-gas flow and, then, condensed into a white product getting collected into the cooling traps near the outlet of the furnace. The as-obtained product is stored inside an argon-filled glove-box. The scanning electron microscopy (SEM) images in Figure 1a show that the sample consists of particles with a relatively homogeneous 
size. This indicates that the most important operating factors, including the properties of the starting precursor, the pyrolysis temperature, the nitrogen flow rate, the residence time and heating rate of the droplet particles, are controlled during processing.

Figure 1. SEM (a), TEM (b) and HRTEM (c) images of samples obtained by spray-pyrolysis of borazine (BZ).

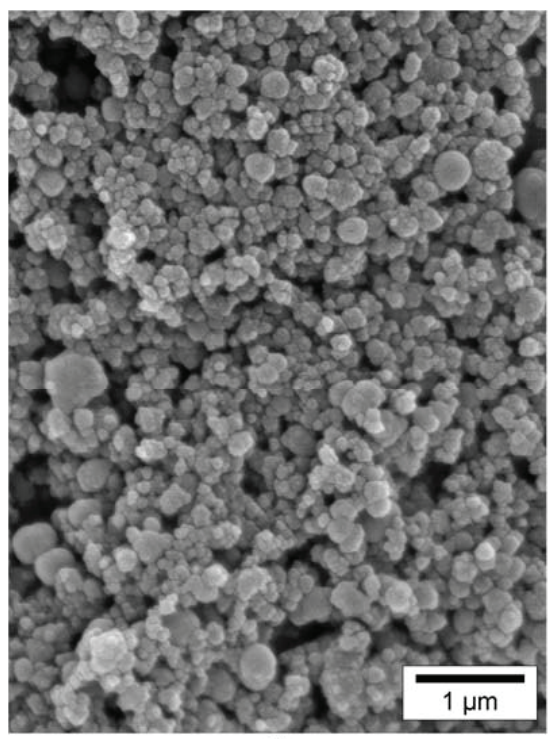

(a)

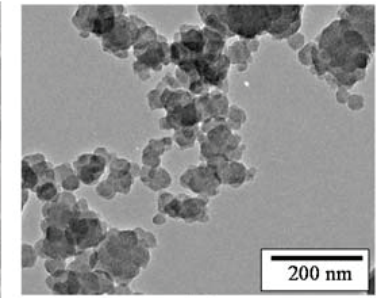

(b)

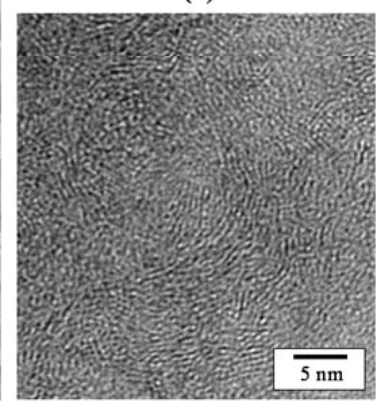

(c)

The low-magnification transmission electron microscope (TEM) bright field image of the sample (Figure 1b) show elementary blocks that are composed of slightly agglomerated nanoparticles (NPs). The particle size ranges from 55 to $120 \mathrm{~nm}$. The high resolution TEM (HRTEM) image (Figure 1c) of the particle core demonstrates that the specimen consists of very fine $\mathrm{BN}$ crystallites in which $s p^{2}$ layers are significantly buckled in a disordered stacking sequence, exhibiting a size corresponding to less than six atomic basal planes, whereas their length does not exceed $50 \AA$. This points to the fact that BN is poorly crystallized similarly to a turbostratic structure. The TEM data are reinforced by the X-ray diffraction (XRD) experiments (Figure 2). The corresponding XRD patterns show very broad peaks at the $h$-BN (002), (100)/(101)/(004) and (110) positions. In particular, the (002) peak slightly shifts to lower angles in such samples, and the (100), (101) and (004) peaks merge into a single broad peak. Finally, the samples displayed a chemical formula of $\mathrm{B}_{1.0} \mathrm{~N}_{0.9}$. Their specific surface area is $34.6 \mathrm{~m}^{2} \mathrm{~g}^{-1}$, and the helium density is $1.95 \mathrm{~g} \mathrm{~cm}^{-3}$, as measured by Brunauer-Emmett-Teller (BET) and helium pycnometry, respectively.

$\mathrm{B}_{1.0} \mathrm{~N}_{0.9}$-NPs are stable in air below $850{ }^{\circ} \mathrm{C}$ in which only surface oxidation proceeds [21]. Here, we report the evolution of the nanostructural organization of $\mathrm{B}_{1.0} \mathrm{~N}_{0.9}$-NPs in the temperature range of $1450-2000{ }^{\circ} \mathrm{C}$ under nitrogen. The XRD patterns in Figure 2 range from $10^{\circ}$ to $90^{\circ}$ for heat-treated $\mathrm{B}_{1.0} \mathrm{~N}_{0.9}$-NPs. 
Figure 2. XRD patterns of borazine-derived $\mathrm{B}_{1.0} \mathrm{~N}_{0.9}-\mathrm{NPs}$ and annealed at a temperature ranging from 1450 to $2000{ }^{\circ} \mathrm{C}$.

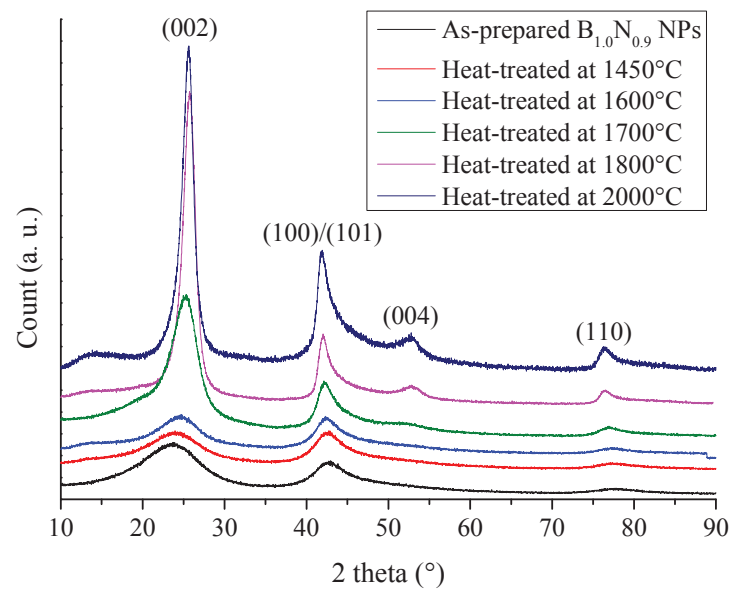

The XRD patterns of samples heat-treated in the temperature range of $1450-1600{ }^{\circ} \mathrm{C}$ display features similar to the ones recorded for $\mathrm{B}_{1.0} \mathrm{~N}_{0.9}-\mathrm{NPs}$, indicating a turbostratic structure. For the sample annealed at $1700{ }^{\circ} \mathrm{C}$, the $(002)$ peak at $25.30^{\circ}$ is sharpened, suggesting that the crystallite size became larger in the $c$-axis direction, although the shoulder-shaped broad feature remained on the low-angle side of the peak. This is also shown for the sharper $(100) /(101) /(004)$ peak, which tends to be separated into the (004) peak and the $(100) /(101)$ peak. The increase of the heat-treatment temperature to $1800{ }^{\circ} \mathrm{C}$ and $2000{ }^{\circ} \mathrm{C}$ resulted in an increased resolution of the XRD patterns. We can clearly distinguish the (002), (100)/(101), (004) and (110) peak positions. According to the sharpening of the $(002)$ and $(100) /(101)$ peaks, we suggest that the crystallite size continuously increased in the $c$ - and $a$-axes directions from $1400^{\circ} \mathrm{C}$ to $2000^{\circ} \mathrm{C}$. However, no clear peaks corresponding to the (102) and (112) planes were observed. These findings tend to demonstrate that $\mathrm{B}_{1.0} \mathrm{~N}_{0.9}$-NPs annealed at $2000{ }^{\circ} \mathrm{C}$ exhibit a turbostratic structure. The variation of the average crystallite size in the $c$-axis from the $(002)$ peak $\left(\overline{\mathrm{L}_{\mathrm{c}}}\right)$ and the interlayer $\mathrm{d}_{002}$ spacing of the samples during heat-treatment is shown in Figure 3. The dimension d002 is calculated from Bragg's law using the diffraction angle of the (002) peak. $\bar{L}_{c}$ represents the average number of stacked layers in the crystallites. The average stack height $\overline{\mathrm{L}_{\mathrm{c}}}$ is calculated from the Scherrer relation $\left(\overline{\mathrm{L}_{\mathrm{c}}}=0.9 \lambda /\left(\mathrm{B}^{2}-\mathrm{B}^{12}\right)^{1 / 2} \cos \theta\right.$, where $\lambda$ is the $\mathrm{CuK}_{\alpha 1}$ wavelength $(\lambda=0.1540 \mathrm{~nm}), \theta$ the Bragg angle of the (002) diffraction peak, B the full width at half maximum intensity (FWHM) of the peak and $\mathrm{B}^{\prime}$ the instrumental contribution).

In the range of $1450{ }^{\circ} \mathrm{C}\left(\overline{\mathrm{L}_{\mathrm{c}}}=1.10 \mathrm{~nm} ; \mathrm{d}_{002}=0.367 \mathrm{~nm}\right)-1600{ }^{\circ} \mathrm{C}\left(\overline{\mathrm{L}_{\mathrm{c}}}=1.42 \mathrm{~nm} ; \mathrm{d}_{002}=0.363 \mathrm{~nm}\right)$, there is no major modification in both the apparent average grain size $\left(\overline{\mathrm{L}}_{\mathrm{c}}(002)\right)$ and the value of the interlayer $\mathrm{d}$-spacing $\mathrm{d}_{002}$. Values are close to those calculated for as-prepared $\mathrm{B}_{1.0} \mathrm{~N}_{0.9}-\mathrm{NPs}$ $\left(\overline{\mathrm{L}_{\mathrm{c}}}=1.06 \mathrm{~nm} ; \mathrm{d}_{002}=0.376 \mathrm{~nm}\right)$. This indicates a relatively high amount of disorder in the structure of the corresponding samples. At $1700{ }^{\circ} \mathrm{C}$, the apparent average grain size increases slightly $\left(\overline{\mathrm{L}_{\mathrm{c}}}=2.23 \mathrm{~nm}\right)$. Although the crystallization state in NPs heat-treated at $1700^{\circ} \mathrm{C}$ is slightly improved, the $\mathrm{BN}$ phase remains poorly ordered as confirmed by the value of $\mathrm{d}_{002}\left(\mathrm{~d}_{002}=0.351 \mathrm{~nm}\right)$, higher than 
that in a $h$-BN crystal $(0.3327 \mathrm{~nm})$. At $1800{ }^{\circ} \mathrm{C}, \overline{\mathrm{L}_{\mathrm{c}}}$ increases to $4.63 \mathrm{~nm}$ and the interlayer d002 spacing is found to be $0.345 \mathrm{~nm}$, which are values characteristic of a turbostratic phase. The minor changes in the XRD patterns of samples heat-treated at $2000{ }^{\circ} \mathrm{C}$ is reflected in the values of $\overline{\mathrm{L}_{\mathrm{c}}}(4.65 \mathrm{~nm})$ and d002 $(0.346 \mathrm{~nm})$. In addition to XRD studies, we investigated TEM (Figure 4) and HRTEM (Figure 5) experiments to follow the evolution of the nanostructural organization in the temperature range of $1450-2000^{\circ} \mathrm{C}$.

Figure 3. Evolution of $\overline{\mathrm{L}_{\mathrm{c}}(002)}$ and $\mathrm{d}_{002} v s$. annealing temperature.

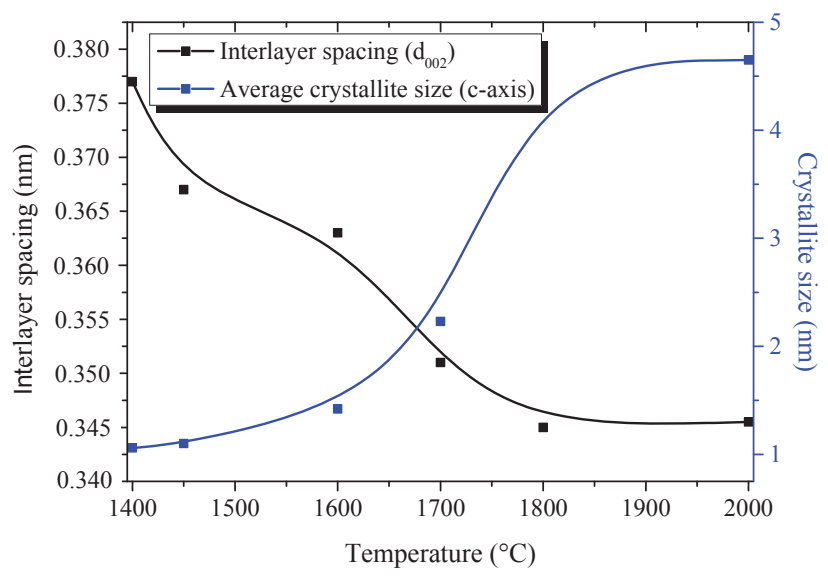

Figure 4. TEM images of the samples annealed at (a) $1450{ }^{\circ} \mathrm{C}$; (b) $1600^{\circ} \mathrm{C}$; (c) $1700{ }^{\circ} \mathrm{C}$; (d) $1800{ }^{\circ} \mathrm{C}$ and (e) $2000{ }^{\circ} \mathrm{C}$.

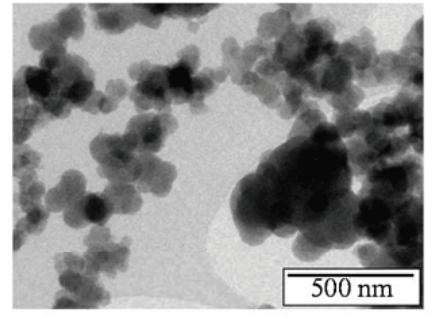

(a)

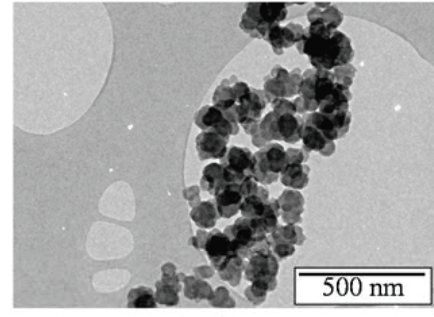

(b)

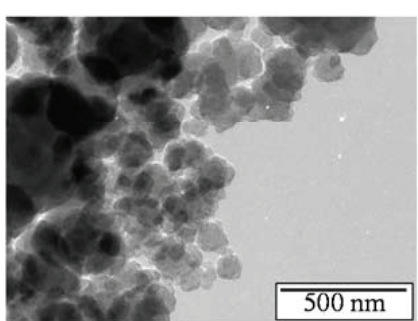

(c)

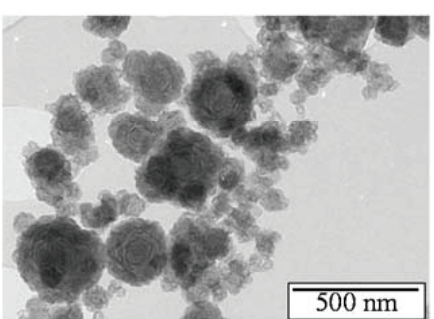

(d)

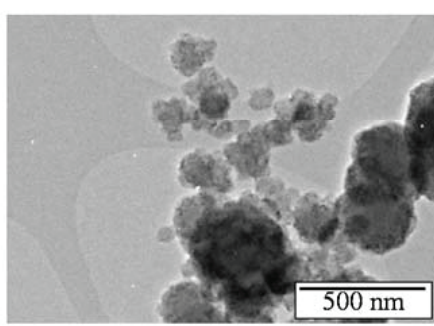

(e) 
Figure 5. HRTEM images of the samples annealed at (a) $1450{ }^{\circ} \mathrm{C}$; (b) $1600{ }^{\circ} \mathrm{C}$; (c) $1700{ }^{\circ} \mathrm{C}$; (d) $1800{ }^{\circ} \mathrm{C}$; (e) $2000{ }^{\circ} \mathrm{C}$; (f) evidence of a core-shell structure generated at $2000{ }^{\circ} \mathrm{C}$.

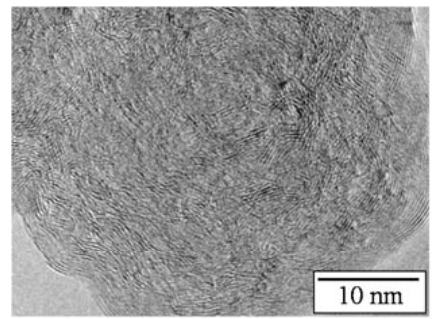

(a)

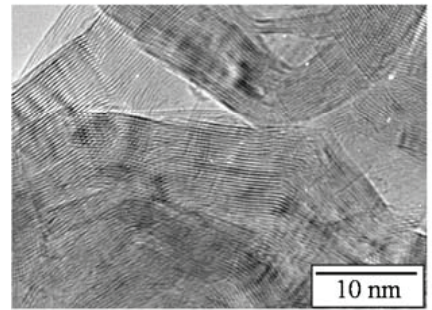

(d)

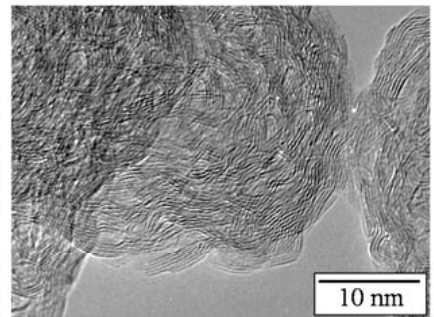

(b)

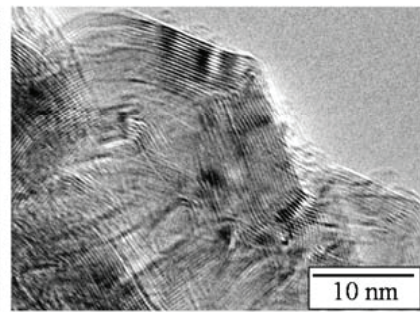

(e)

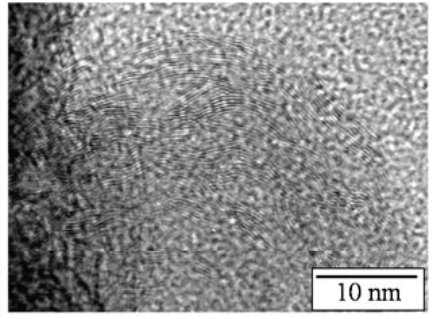

(c)

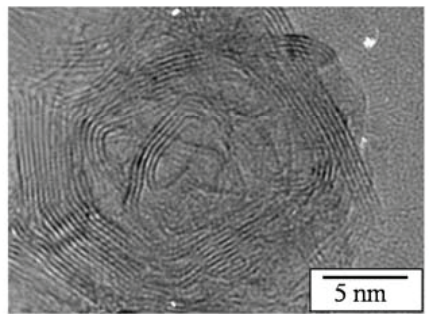

(f)

The annealed samples form elementary blocks composed of nanosized particles that are round in shape and slightly agglomerated. Both the average size of annealed particles and the agglomeration seem to increase with the temperature of the annealing, which is in good agreement. This is clear for the samples annealed at $1800{ }^{\circ} \mathrm{C}$ (Figure $4 \mathrm{~d}$ ) and $2000{ }^{\circ} \mathrm{C}$ (Figure 4e), respectively. We therefore extended our analysis, by performing high resolution TEM (HRTEM), in order to refine/emphasize the structural information.

Figure 5 reports HRTEM images of the same samples.

Clear differences appear between the samples annealed in the range of 1450-2000 ${ }^{\circ} \mathrm{C}$. After heat-treatment to $1450{ }^{\circ} \mathrm{C}$ (Figure 5a), the sample displays a turbostratic BN structure with more distinct (002) layers in comparison to the nanostructure observed in pristine $\mathrm{B}_{1.0} \mathrm{~N}_{0.9}$-NPs (Figure 1c). In the sample annealed at $1600{ }^{\circ} \mathrm{C}$ (Figure 5b) and $1700{ }^{\circ} \mathrm{C}$ (Figure 5c), we can also observe the formation of nanodomains made of BN layers surrounding voids. The HRTEM image reveals the formation of concentric shelled nanodomains. The lattices of these BN nanostructures have a local interlayer spacing of $3.51 \AA$. Annealing at a temperature of $1800{ }^{\circ} \mathrm{C}$ (Figure 5d) and $2000{ }^{\circ} \mathrm{C}$ (Figure 5e,f) leads to hollow-cored BN-NPs that are strongly facetted, forming polygonal particles with an interlayer spacing of $3.34 \AA$. We investigated the potential of samples heat-treated at $2000{ }^{\circ} \mathrm{C}$ to confine $\mathrm{H}_{2}$ storage materials.

\subsection{Hydrogen Storage Applications}

Ammonia borane $(\mathrm{AB})$ is a white crystalline solid that was first prepared by Shore and Parry in 1955 [23]. Over the past decade, this compound has attracted considerable attention as portable 
hydrogen storage materials, according to its high gravimetric hydrogen contents ( $\mathrm{ca}$. $20 \%$ by weight) [24-29]. A very pertinent review dedicated to this compound and related derivatives as dihydrogen sources was proposed by Staubitz et al. in 2010 [29].

In the pristine state, $\mathrm{AB}$ is almost stable under inert conditions up to about $100{ }^{\circ} \mathrm{C}$ and decomposes within the range $100-200{ }^{\circ} \mathrm{C}$ through a two-step exothermic process where two equivalent $\mathrm{H}_{2}$, as well as undesired by-products, such as borazine $\mathrm{B}_{3} \mathrm{~N}_{3} \mathrm{H}_{6}$ and $\mathrm{NH}_{3}$, are evolved [24,25]. This decomposition suffers from three important problems: (1) the process is exothermic, which means that the storage reversibility is thermodynamically impossible in acceptable operating conditions; (2) the dehydrogenation temperature is too high for the portable/mobile application prospects; (3) the emission of undesired by-products is detrimental, as they are incompatible with the use of proton exchange membrane fuel cell (PEMFC) [27].

A promising solution seems to be the decrease of the particle size at the nanoscale $(<10 \mathrm{~nm})$ via confinement of the borane in a porous compound (i.e., scaffold) [30]. As an illustration, Gutowska et al. showed that AB confined in the mesoporosity of silica SBA (Santa Barbara Amorphous)-15 has improved dehydrogenation behavior in comparison to the pristine hydride, with an onset at $70{ }^{\circ} \mathrm{C}$ and the liberation of borazine-free $\mathrm{H}_{2}$ [31]. The destabilization of $\mathrm{AB}$ is generally explained by two phenomena. The first one is the nanosizing of the hydride particle. At the nanoscale, both kinetics and thermodynamics might be altered by both size and interface effects. In fact, the surface energy value could be different as a result of the interactions between the active confined material and the scaffold. The second phenomenon is associated with $\mathrm{H}^{\delta+} \cdots \mathrm{H}^{\delta-}$ surface interactions, with $\mathrm{H}^{\delta^{-}}$of the $\mathrm{BH}_{3}$ moiety of $\mathrm{AB}$ and $\mathrm{H}^{\delta+}$ belonging to surface/terminal hydroxyl groups $(-\mathrm{O}-\mathrm{H})$ generally found on carbonaceous or oxide nano-scaffolds. Such acid-base interactions enhance $\mathrm{H}_{2}$ release, but usually lead to an unstable material at room conditions [25].

An improved strategy we recently demonstrated is to use nano-scaffolds free of reactive surface groups [22]. For that purpose, we used the $\mathrm{B}_{1.0 \mathrm{~N}} \mathrm{~N}_{0.9}$-NPs annealed at $2000{ }^{\circ} \mathrm{C}$, which we labeled $\mathrm{B}_{1.0} \mathrm{~N}_{0.9}$-NP2000. As measured by energy dispersive X-ray spectrometry (EDX), boron, nitrogen and oxygen contents are 43.55, 55.7 and $0.75 \mathrm{wt} \%$, respectively. Unfortunately, they exhibit a Brunauer-Emmett-Teller (BET)-specific surface area of $21.8 \mathrm{~m}^{2} \cdot \mathrm{g}^{-1}$, which is low to achieve the nanoconfinement of AB. Therefore, we applied a ball-milling process of this sample to tentatively increase the specific surface area, leading to the sample labeled $\mathrm{B}_{1.0} \mathrm{~N}_{0.9}$-NP2000BM. In comparison to $\mathrm{B}_{1.0} \mathrm{~N}_{0.9}$-NP2000, the sample $\mathrm{B}_{1.0} \mathrm{~N}_{0.9}$-NP2000BM shows a considerably increased BET-specific surface area with $200.5 \mathrm{~m}^{2} \cdot \mathrm{g}^{-1}$ and a total pore volume of $0.424 \mathrm{~cm}^{3} \cdot \mathrm{g}^{-1}$ as measured by the Barrett-Joyner-Halenda (BJH) analysis. As a result of the ball-milling, the HRTEM images (Figure 6a,b) of the sample showed that cleavage of the walls occurred through the basal planes. In addition, Figure $6 \mathrm{c}$ show that the stacking sequence can in some cases be disordered similarly to those of $t$-BN after ball-milling.

As a result of the BET and TEM investigations, we successfully demonstrated that the walls of the hollow-cored BN-NPs could be opened to provide porosity after ball-milling. 
Figure 6. HRTEM images of the sample $\mathrm{B}_{1.0} \mathrm{~N}_{0.9}-\mathrm{NP} 2000 \mathrm{BM}$ evidencing in (a) and (b), a cleavage of the walls in the area delimited by the white arrows, and in (c), a disordering of the stacking sequence.

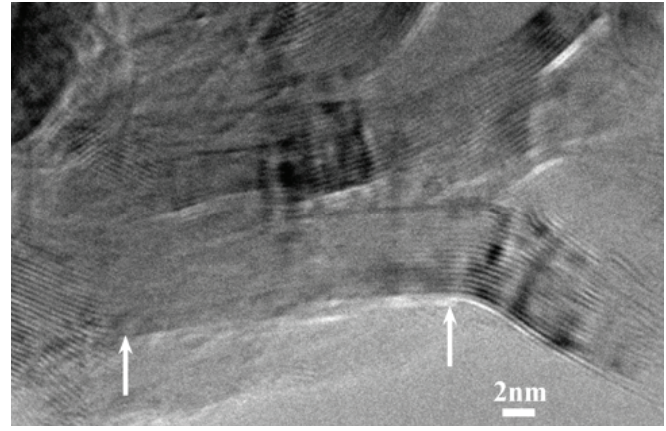

(a)

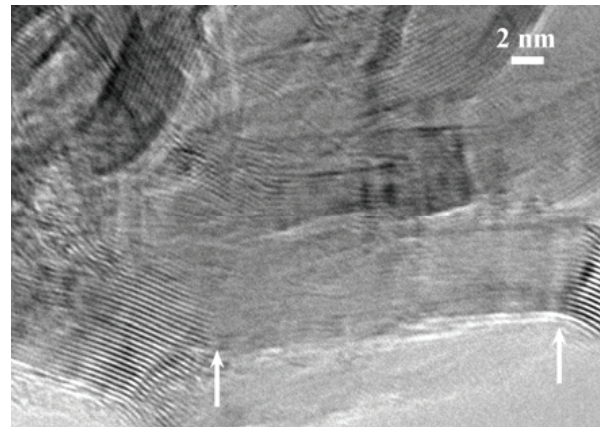

(b)

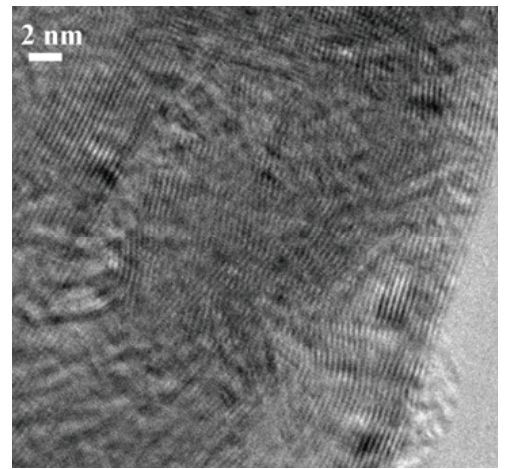

(c)

Hydrogen storage materials can be confined within porous scaffolds by melt infiltration (if the active hydrogen storage material melts and do not decompose) or solution infiltration. In our procedure, a solution of $\mathrm{AB}$ in tetrahydrofuran (THF) was infiltrated into the framework of the sample $\mathrm{B}_{1.0} \mathrm{~N}_{0.9}$-NP2000BM according to an optimized procedure described elsewhere [25]. A nanocomposite labeled $\mathrm{AB} @ \mathrm{~B}_{1.0} \mathrm{~N}_{0.9}-\mathrm{NP} 2000 \mathrm{BM}$ was formed. It was stored at $3-4{ }^{\circ} \mathrm{C}$. The successful impregnation of $\mathrm{AB}$ in $\mathrm{B}_{1.0} \mathrm{~N}_{0.9}-\mathrm{NP} 2000 \mathrm{BM}$ was followed by $\mathrm{N}_{2}$ adsorption/desorption analysis of the nanocomposite. A BET-specific surface area of $6.7 \mathrm{~m}^{2} \cdot \mathrm{g}^{-1}$ and a total pore volume of $0.023 \mathrm{~cm}^{3} \cdot \mathrm{g}^{-1}$ are measured, which demonstrates that $\mathrm{AB}$ was inserted into the hollow core and blocked the pores of the nano-scaffolds. More interesting is that the decomposition of $\mathrm{AB}$ is down to $81{ }^{\circ} \mathrm{C}$ (compared to $110^{\circ} \mathrm{C}$ for the pristine $\mathrm{AB}$ in our conditions) and that a major evolution of $\mathrm{H}_{2}$ is identified by $\mathrm{MS}$. In our experimental conditions, the only by-product was identified to be $\mathrm{NH}_{3}$ above $80{ }^{\circ} \mathrm{C}$. At $80{ }^{\circ} \mathrm{C}$, a weight loss of $1.7 \mathrm{wt} \%$ was measured, which means an effective gravimetric hydrogen storage capacity of $3.4 \mathrm{wt} \%$ by considering a weight ratio equal to $1: 1$ in $\mathrm{AB} @ \mathrm{~B}_{1.0} \mathrm{~N}_{0.9}-\mathrm{NP} 2000 \mathrm{BM}$.

Our results confirmed the remarkable benefit of hollow-cored BN-NPs on the dehydrogenation behavior of $\mathrm{AB}$. The performance is comparable to the dehydrogenation results of $\mathrm{AB}$ confined into a magnesium metal organic framework (MOF) [32,33] or nickel MOF [34], whereas only nanoconfinement 
is considered here. Most interesting, the MS results suggest that there is no detectable trace of borazine as a gaseous by-product. Another important observation standing from the thermogravimetric analysis coupled mass spectrometry (TGA-MS) result is that AB@ $1 \mathrm{~B}_{1.0} \mathrm{~N}_{0.9}-\mathrm{NP} 2000 \mathrm{BM}$ is stable at room conditions. Accordingly, the stability of $\mathrm{AB} @ \mathrm{~B}_{1.0} \mathrm{~N}_{0.9}-\mathrm{NP} 2000 \mathrm{BM}$ at $<40{ }^{\circ} \mathrm{C}$ is clearly attributed to the absence of surface $\mathrm{H}^{\delta+}$, and the improvement of the dehydrogenation properties of $\mathrm{AB}$ in $\mathrm{AB} @ \mathrm{~B}_{1.0} \mathrm{~N}_{0.9}-\mathrm{NP} 2000 \mathrm{BM}$ can be exclusively ascribed to the effect of nanoconfinement.

\section{Experimental Section}

The synthesis of borazine was carried out in an argon atmosphere, using argon/vacuum lines and Schlenk-type flasks. Argon (>99.995\%) was purified by passing through successive columns of phosphorus pentoxide (Sigma-Aldrich, Saint Quentin, France), sicapent ${ }^{\mathrm{TM}}$ (Millipore S.A.S, Molsheim, France) and copper oxide-based catalysts (Sigma-Aldrich, Saint Quentin, France). The Schlenk flasks were dried at $120{ }^{\circ} \mathrm{C}$ overnight before pumping under vacuum and before filling with argon for the synthesis. Sodium borohydride $\left(\mathrm{NaBH}_{4}, \geq 98.5 \%\right.$, powder from Sigma-Aldrich, Saint Quentin, France), ammonium sulfate $\left(\left(\mathrm{NH}_{4}\right)_{2} \mathrm{SO}_{4}, \geq 99.0 \%\right.$ from Sigma-Aldrich (Saint Quentin, France) and tetraethylene glycol dimethyl ether $\left(\mathrm{CH}_{3} \mathrm{O}\left(\mathrm{CH}_{2} \mathrm{CH}_{2} \mathrm{O}\right)_{4} \mathrm{CH}_{3}, 99.0 \%\right.$, from Sigma-Aldrich (Saint Quentin, France) were used as-received. It should be mentioned that ammonium sulfate was dried at $120^{\circ} \mathrm{C}$ inside an oven for three days, then put under vacuum during cooling for $1 \mathrm{~h}$. Manipulation of the chemical products was made inside an argon-filled glove box (Jacomex BS521; Dagneux, France) dried with phosphorus pentoxide.

Borazine Synthesis: The operating procedure, adapted from the literature [18], was previously reported by our group [19]. FTIR (Caesium Iodide $(\mathrm{CsI})$ windows $\left./ \mathrm{cm}^{-1}\right)$ : $(\mathrm{N}-\mathrm{H})=3451$ medium; $(\mathrm{B}-\mathrm{H})=2509$ medium; $(\mathrm{B}-\mathrm{N})=1454$ small; $(\mathrm{B}-\mathrm{N}-\mathrm{B})=897$ medium. ${ }^{1} \mathrm{H}$ NMR $\left(300 \mathrm{MHz} / \mathrm{CDCl}_{3} / \mathrm{ppm}\right):=3.30-5.35$ (quadruplet, $3 \mathrm{H}, \mathrm{B} H$ ), 5.35-6.05 (triplet, $\left.3 \mathrm{H}, \mathrm{NH}\right) .{ }^{11} \mathrm{~B} \mathrm{NMR}$ (96.29 MHz/C6 $\left.\mathrm{D}_{6} / \mathrm{ppm}\right):=30.1$ (br).

Nanoparticle Preparation: The experimental set-up is composed of a nebulized spray generator (RBI, Meylan, France), in which the spray is generated by a piezoelectric device (barium titanate). Frequency $(800 \mathrm{kHz})$ and power $(100 \mathrm{~W})$ alimentations are adjusted to obtain the aerosol. The aerosol temperature is first held at $15^{\circ} \mathrm{C}$ by a regulated water circulation to avoid borazine evaporation and/or condensation. The piezoelectric device generates an ultrasound beam, which is directed to the liquidgas interface; a fountain formed at the surface followed by the generation of the spray, resulting from vibrations at the liquid surface and cavitations at the gas-liquid interface.

The borazine was directly introduced in the aerosol generating chamber under nitrogen, then aerosolized and carried to the pyrolysis furnace with a $0.5 \mathrm{~mL} \cdot \mathrm{min}^{-1}$ nitrogen flow rate. The thermal decomposition of borazine was performed in a hot alumina tube containing an isothermal zone of $0.1 \mathrm{~m}$ in length. The fast heating rate implies gaseous species generation leading to powder formation by a chemical vapor condensation route. The particles were finally trapped into two collectors placed before the vacuum pump and containing filter-barriers made of microporous alumina (pore size of $1 \mu \mathrm{m}$ ). Yield was estimated to be $0.22 \mathrm{~g} \cdot \mathrm{min}^{-1}$. After synthesis, the particles are stored inside an argon-filled glove-box. In a typical experiment, $27 \mathrm{~mL}(21.9 \mathrm{~g})$ of borazine is used to produce $6.5 \mathrm{~g}$ of $\mathrm{B}_{1.0} \mathrm{~N}_{0.9}$-NPs. However, the exact yield is difficult to estimate, because of the design of the 
spray-pyrolysis system. A non-negligible/considerable quantity of powders, deposited in the furnace tube, cannot be recovered. To study the evolution of their crystallization degree, $2 \mathrm{~g}$ of the $\mathrm{B}_{1.0} \mathrm{~N}_{0.9}-\mathrm{NPs}_{\mathrm{s}}$ are placed into boron nitride boats and then introduced in a graphite furnace (Gero 5 Model HTK 8). The furnace chamber is subsequently suctioned with a pump charged with nitrogen before heating. A cycle of ramping at $10{ }^{\circ} \mathrm{C} \cdot \mathrm{min}^{-1}$ is used to heat the sample to the desired temperature (in the range 1400-2000 ${ }^{\circ} \mathrm{C}$ ) with a holding time of $1 \mathrm{~h}$, before cooling down to $\mathrm{RT}$ at $10^{\circ} \mathrm{C} \cdot \mathrm{min}^{-1}$. Chemical analysis found (wt\%): $\mathrm{B}, 50.0 ; \mathrm{N}, 49.4 ; \mathrm{O}, 0.6$. The milling process of $\mathrm{B}_{1.0} \mathrm{~N}_{0.9}-\mathrm{NP} 2000$ is performed under inert condition (argon) with a planetary ball-miller (Retsch PM100; Haan, Germany). The described process has been optimized (in terms of mass, ratio balls/BN, time, rotation) to our conditions. Typically, degassed $\mathrm{B}_{1.0} \mathrm{~N}_{0.9}-\mathrm{NP} 2000$ (at $150{ }^{\circ} \mathrm{C}$ under dynamic vacuum for $24 \mathrm{~h}$ ) is introduced into a stainless steel reactor $\left(25 \mathrm{~mL}\right.$ ). Balls in stainless steel are added (weight ratio balls: $\mathrm{B}_{1.0} \mathrm{~N}_{0.9}-\mathrm{NP} 2000$ of 20). The milling process is performed at $600 \mathrm{rpm}$ for $1 \mathrm{~h}$. The as-obtained $\mathrm{B}_{1.0} \mathrm{~N}_{0.9}-\mathrm{NP} 2000 \mathrm{BM}$ is finally sieved.

The infiltration of ammonia borane is performed as follows: the host material $\mathrm{B}_{1.0} \mathrm{~N}_{0.9}$-NP2000BM (100 mg) is degassed at $150{ }^{\circ} \mathrm{C}$ under dynamic vacuum for $24 \mathrm{~h}$ in a Schlenk tube and then cooled to $0{ }^{\circ} \mathrm{C}$. In an argon-filled glove box, a concentrated solution of ammonia borane (100 mg, 97\%; Sigma Aldrich, Saint Quentin, France) is prepared using $0.5 \mathrm{~mL}$ of anhydrous THF (Sigma Aldrich, Saint Quentin, France). The ammonia borane solution is injected into the Schlenk tube containing $\mathrm{B}_{1.0} \mathrm{~N}_{0.9}$-NP2000BM kept under static vacuum and at $0{ }^{\circ} \mathrm{C}$. By capillary action, the ammonia borane solution fills the channels of the host rapidly, which is evidenced by vigorous effervescence. When the effervescence stops, the sample is put under ultrasonic treatment for $20 \mathrm{~min}$ at $0{ }^{\circ} \mathrm{C}$. Finally, the as-obtained sample $\mathrm{AB} @ \mathrm{~B}_{1.0} \mathrm{~N}_{0.9}-\mathrm{NP} 2000 \mathrm{BM}$ (weight ratio $\mathrm{B}_{1.0} \mathrm{~N}_{0.9}-\mathrm{NP} 2000 \mathrm{BM}: \mathrm{AB}$ of 1 ) is dried under dynamic vacuum for $48 \mathrm{~h}$ at $0{ }^{\circ} \mathrm{C}$. The composite samples obtained are denoted $\mathrm{AB} @ \mathrm{~B}_{1.0} \mathrm{~N}_{0.9}-\mathrm{NP} 2000 \mathrm{BM}$. Samples are transferred in an argon-filled vial and then stored in a fridge at $3-4{ }^{\circ} \mathrm{C}$.

Characterizations: The $\mathrm{B}_{1.0} \mathrm{~N}_{0.9}$-NPs and annealed samples are first mounted on carbon film-covered stainless pads for scanning electron microscopy (SEM, Hitachi S4800, Tokyo, Japan) including Energy Dispersive X-ray Spectroscopy (EDX, EDAX/TSL Genesis 4000, Tokyo, Japan). Due to the insulating properties of $\mathrm{BN}$, the samples are sputtered with $10 \mathrm{~nm}$ of a $\mathrm{Pd} / \mathrm{Au}$ mixture to prevent charging during SEM observations. In parallel, the same samples are ultrasonicated in ethanol, and the resulting solution is afterwards deposited on a collection of hollow carbon-film-covered copper grids for transmission electron microscopy (TEM, TOPCON 002B working at $200 \mathrm{kV}$, Tokyo, Japan) observation. Samples were characterized using a Philips PW 3040/60 X'Pert PRO X-ray diffraction system (Eindhoven, The Netherland). Powder samples are prepared by placing $\sim 100 \mathrm{mg}$ on the XRD sample holder (PVC), and the sintered pieces were put down on the XRD sample holder for data collection. $\mathrm{Cu} \mathrm{K} \alpha(\lambda=1.54 \AA)$ radiation with a Ni filter was used with a working voltage and a current of $40 \mathrm{kV}$ and $30 \mathrm{~mA}$, respectively. Scans were continuous from $2 \theta=10^{\circ}-90^{\circ}$ with a time per step of $0.85 \mathrm{~s}$ in increments of $0.017^{\circ}$. Peak positions and relative intensities were characterized by comparison with JCPDS (Joint Committee on Powder Diffraction Standards) files of the standard material (JCPDS card No 34-0421). Debye-Scherrer line broadening was used to calculate the average crystallite sizes from each XRD pattern. The transmission electron microscopy (TEM) studies of 
$\mathrm{B}_{1.0} \mathrm{~N}_{0.9}$-NP2000BM samples were carried out with a JEOL (Tokyo, Japan) GmbH 2010F transmission electron microscope $(\mathrm{Cs}=1 \mathrm{~mm})$ operating at $200 \mathrm{kV}$. The characterization of the samples was performed by $\mathrm{N}_{2}$ adsorption/desorption (Sorptomatic 1990 Series, Thermo Fisher Scientific Inc, Waltham, MA, USA). Thermogravimetric analysis (TGA) measurements (repeated at least three times to ensure the reproducibility of the results) were performed with a Mettler Toledo TGA/SDTA 851e (Schwerzenbach, Switzerland) under the following conditions: sample mass 9-10 $\mathrm{mg}$, aluminum crucible of $100 \mu \mathrm{L}$ with a pinhole, heating rate of $5^{\circ} \mathrm{C} \cdot \mathrm{min}^{-1}$, temperature range 25-200 ${ }^{\circ} \mathrm{C}$ and atmosphere of $\mathrm{N}_{2}\left(60 \mathrm{~mL} \cdot \mathrm{min}^{-1}\right)$. The purity of $\mathrm{H}_{2}$ was analyzed by mass spectrometry (Canon Anelva Corporation MQA200TS, Tokyo, Japan) coupled to the TGA apparatus.

\section{Conclusions}

This article reviews our recent advancements in the synthesis and energy application of nanostructured boron nitride. Such materials exhibit chemical and physical properties that are significantly different from those of bulk and microsized materials. In our approach, we discussed our recent strategy based on borazine, which has been used as a vapor phase pyrolysis precursor for the synthesis and fabrication of BN nanoparticles. In particular, we demonstrated the possibility of tailoring the nanostructure of these nanoparticles by a further annealing process at high temperature $\left(2000{ }^{\circ} \mathrm{C}\right)$, leading to hollow-cored BN-NPs that are strongly facetted, forming polygonal particles with an interlayer spacing of $3.34 \AA$. The ball-milling of these nanostructures allowed developing the specific surface area of the material while hollow-cored BN-NPs porous shell structures were obtained. They show a BET-specific surface area of $200.5 \mathrm{~m}^{2} \cdot \mathrm{g}^{-1}$, a total pore volume of $0.287 \mathrm{~cm}^{3} \cdot \mathrm{g}^{-1}$ and a narrow pore size distribution centered at $3.5 \mathrm{~nm}$. They were used as nano-scaffolds of ammonia borane in order to improve its dehydrogenation properties to form a nanocomposite able to liberate pure $\mathrm{H}_{2}$ in the temperature range $40-80{ }^{\circ} \mathrm{C}$ in our conditions. The only trace of by-product being detected at $>80{ }^{\circ} \mathrm{C}$ is ammonia. Considering the regenerability of ammonia borane [35], our results suggest that our composite material is a safe and practical hydrogen storage material. This improvement is exclusively ascribed to the nanoconfinement effect.

\section{Acknowledgments}

The authors acknowledge Vincent Salles for spray-pyrolysis and Arnaud Brioude for the TEM observation of nanoparticles and samples annealed in the temperature range of $1450-2000{ }^{\circ} \mathrm{C}$ before ball-milling.

\section{Author Contributions}

The findings in this manuscript are part of Georges Moussa thesis work. Chrystelle Salameh performed borazine synthesis. Philippe Miele, Umit B. Demirci and Samuel Bernard advised the thesis and directed the research. Alina Bruma and Sylvie Malo guided the TEM experiments of the sample $\mathrm{B}_{1.0} \mathrm{~N}_{0.9}$-NP2000BM. The manuscript was written through contributions of all authors. All authors have given approval to the final version of the manuscript. 


\section{Conflicts of Interest}

The authors declare no conflict of interest.

\section{References}

1. Paine, R.T.; Narula, C.K. Synthetic route to boron nitride. Chem. Rev. 1990, 90, 73-91.

2. Wu, J.; Han, W.-Q.; Walukiewicz, W.; Ager, J.W., III; Shan, W.; Haller, E.E.; Zettl, A. Raman spectroscopy and time-resolved photoluminescence of $\mathrm{BN}$ and $\mathrm{B}_{x} \mathrm{C}_{y} \mathrm{~N}_{z}$ nanotubes. Nano Lett. 2004, 4, 647-650.

3. Watanabe, K.; Tanigushi, T.; Kanda, H. Direct-bandgap properties and evidence for ultraviolet lasing of hexagonal boron nitride single crystal. Nat. Mater. 2004, 3, 404-409.

4. Kubota, Y.; Watanabe, K.; Tsuda, O.; Tanigushi, T. Deep ultraviolet light-emitting hexagonal boron nitride synthesized at atmospheric pressure. Science 2007, 317, 932-934.

5. Macnaughton, J.B.; Moewes, A.; Wilks, R.G.; Zhou, X.T.; Sham, T.K.; Tanigushi, T.; Watanabe, K.; Chan, C.Y.; Zhang, W.J.; Bello, I.; et al. Electronic structure of boron nitride single crystals and films. Phys. Rev. B 2005, 72, 195113:1-195113:8.

6. Zhong, W.; Wang, S.; Li, J.; Bechelany, M.C.; Ghisleni, R.; Rossignol, F.; Balan, C.; Chartier, T.; Bernard, S.; Miele, P.; et al. Design of carbon fibre reinforced boron nitride matrix composites by vacuum-assisted polyborazylene transfer moulding and pyrolysis. J. Eur. Ceram. Soc. 2013, 33, 2979-2992.

7. Balmain, W.H. Bemerkungen über die Bildung von Verbindungen des Bors und Siliciums mit Stickstoff und gewissen Metallen. J. Prakt. Chem. 1842, 27, 422-430 (In German).

8. Lipp, A.; Schwetz, K.A.; Hunold, K. Hexagonal boron nitride: Fabrication, properties and applications. J. Eur. Ceram. Soc. 1989, 5, 3-9.

9. Lei, W.; Portehault, D.; Liu, D.; Qin, S.; Chen, Y. Porous boron nitride nanosheets for effective water cleaning. Nat. Commun. 2013, 4, 1777-1783.

10. Rousseas, M.; Goldstein, A.P.; Mickelson, W.; Worsley, M.A.; Woo, L.; Zettl, A. Synthesis of highly crystalline sp2-bonded boron nitride aerogels. ACS Nano 2013, 7, 8540-8546.

11. Li, J.; Xiao, X.; Xu, X.; Lin, J.; Huang, Y.; Xue, Y.; Jin, P.; Zou, J.; Tang, C. Activated boron nitride as an effective adsorbent for metal ions and organic polluants. Nature 2013, 3, 3208-3214.

12. Weng, Q.; Wang, X.; Zhi, C.; Bando, Y.; Golberg, D. Boron nitride porous microbelts for hydrogen storage. ACS Nano 2013, 7, 1558-1565.

13. Siria, A.; Poncharal, P.; Biance, A.-L.; Fulcrand, R.; Blasé, X.; Purcell, S.T.; Bocquet, L. Giant osmotic energy conversion measured in a single transmembrane boron nitride nanotube. Nature 2013, 494, 455-458.

14. Bernard, S. Design, Processing and Properties of Ceramic Materials from Preceramic Precursors in Materials Science and Technologies; Nova Publishers: New York, NY, USA, 2012.

15. Alauzun, J.G.; Ungureanu, S.; Brun, N.; Bernard, S.; Miele, P.; Backov, R.; Sanchez, C. Novel Monolith-type Boron Nitride Hierarchical Foams Obtained through Integrative Chemistry. J. Mater. Chem. 2011, 21, 14025-14030. 
16. Termoss, H.; Toury, B.; Payan, S.; Brioude, A.; Bernard, S.; Cornu, D.; Vallette, S.; Benayoun, S.; Miele, P. Preparation of boron nitride-based coatings on metallic substrates via infrared irradiation of dip-coated polyborazylene. J. Mater. Chem. 2009, 19, 2671-2674.

17. Stock, A.; Pohland, E. Borwasserstoffe, IX.: B $3 \mathrm{~N}_{3} \mathrm{H}_{6}$. Ber. Dtsch. Chem. Ges. 1926, 59(B), 2215-2223 (In German).

18. Wideman, T.; Sneddon, L.G. Convenient procedures for the laboratory preparation of borazine. Inorg. Chem. 1995, 34, 1002-1003.

19. Salles, V.; Bernard, S.; Li, J.; Brioude, A.; Chehaidi, S.; Foucaud, S.; Miele, P. Design of Highly Dense Boron Nitride by the Combination of Spray-Pyrolysis of Borazine and Additive-free Sintering of Derived Ultrafine Powders. Chem. Mater. 2009, 21, 2920-2929.

20. Bernard, S.; Salles, V.; Li, J.; Brioude, A.; Bechelany, M.; Demirci, U.B.; Miele, P. High-Yield Synthesis of Hollow Boron Nitride Nano-Polyhedrons. J. Mater. Chem. 2011, 21, 8694-8699.

21. Salles, V.; Bernard, S.; Chiriac, R.; Miele, P. Structural and Thermal Properties of Boron Nitride Nanoparticles. J. Eur. Ceram. Soc. 2012, 32, 1867-1871.

22. Moussa, G.; Demirci, U.B.; Malo, S.; Bernard, S.; Miele, P. Boron Nitride Nanopolyhedrons with hollow core@Mesoporous Shell structure: From Design to solid-state hydrogen storage application. J. Mater. Chem. A 2014, 2, 7717-7722.

23. Shore, S.G.; Parry, R.W. The crystalline compound ammonia boraone $\mathrm{H}_{3} \mathrm{NBH}_{3}$. J. Am. Chem. Soc. 1955, 77, 6084-6085.

24. Demirci, U.B.; Bernard, S.; Chiriac, R.; Toche, F.; Miele, P. Hydrogen release by thermolysis of ammonia borane $\mathrm{NH}_{3} \mathrm{BH}_{3}$ and then hydrolysis of its by-product $\left[\mathrm{BNH}_{x}\right]$. J. Power Sources 2011, 196, 279-286.

25. Moussa, G.; Bernard, S.; Demirci, U.B.; Chiriac, R.; Miele, P. Room-temperature hydrogen release from activated carbon-confined ammonia borane. Int. J. Hydr. Energy 2012, 37, 13437-13445.

26. Stephens, F.H.; Pons, V.; Baket, R.T. Ammonia-Borane: The Hydrogen Source par excellence. Dalton Trans. 2007, 2613-2626.

27. Hamilton, C.W.; Baker, R.T.; Staubitz, A.; Manners, I. B-N compounds for chemical hydrogen storage. Chem. Soc. Rev. 2009, 38, 279-293.

28. Sanyal, U.; Demirci, U.B.; Jagirdar, B.R.; Miele, P. Hydrolysis of ammonia borane as hydrogen source: Fundamental issues and potential solutions towards implementation. ChemSusChem. 2011, 4, 1731-1739.

29. Staubitz, A.; Robertson, A.P.M.; Manners, I. Ammonia-borane and related compounds as dihydrogen sources. Chem. Rev. 2010, 110, 1079-1124.

30. De Jong, P.E.; Adelhelm, P. Nanosizing and nanoconfinement: New strategies towards meeting hydrogen storage goals. ChemSusChem. 2010, 3, 1332-1348.

31. Gutowska, A.; Li, L.; Shin, Y.; Wang, C.M.; Li, X.S.; Linehan, J.C.; Smith, R.S.; Kay, B.D.; Schmid, B.; Shaw, W.; et al. Nanoscaffold Mediates Hydrogen Release and the Reactivity of Ammonia Borane. Angew. Chem. Int. Ed. 2005, 44, 3578-3582.

32. Gadipelli, S.; Ford, J.; Zhou, W.; Wu, H.; Udovic, T.J.; Yildirim, T. Nanoconfinement and catalytic dehydrogenation of ammonia borane by magnesium-metal-organic-framework-74. Chem. Eur. J. 2011, 17, 6043-6047. 
33. Srinivas, G.; Travis, W.; Ford, J.; Wu, H.; Guo, Z.-X.; Yildirim, T. Nanoconfined ammonia borane in a flexible metal-organic framework Fe-MIL-53: Clean hydrogen release with fast kinetics. J. Mater. Chem. A 2013, 1, 4167-4172.

34. Peng, Y.; Ben, T.; Jia, Y.; Yang, D.; Zhao, H.; Qiu, S.; Yao, X. Dehydrogenation of Ammonia Borane Confined by Low-Density Porous Aromatic Framework. J. Phys. Chem. C 2012, 116, 25694.

35. Sutton, A.D.; Burrell, A.K.; Dixon, D.A.; Garner, E.B., III; Gordon, J.C.; Nakagawa, T.; Ott, K.C.; Robinson, J.P.; Vasiliu, M. Regeneration of ammonia borane spent fuel by direct reaction with hydrazine and liquid ammonia. Science 2011, 331, 1426-1429. 


\title{
Design of Experiments: Optimizing the Polycarboxylation/Functionalization of Tungsten Disulfide Nanotubes
}

\section{Daniel Raichman, David Strawser and Jean-Paul Lellouche}

\begin{abstract}
Design of experiments (DOE) methodology was used to identify and optimize factors that influence the degree of functionalization (polycarboxylation) of WS2 INTs via a modified acidic Vilsmeier-Haack reagent. The six factors investigated were reaction time, temperature and the concentrations of 2-bromoacetic acid, WS $\mathrm{WN}_{2}$ INT, silver acetate and DMF. The significance of each factor and the associated interactive effects were evaluated using a two-level factorial statistical design in conjunction with statistical software (MiniTab $\left.{ }^{\circledR} 16\right)$ based on quadratic programming. Although statistical analysis indicated that no factors were statistically significant, time, temperature and concentration of silver acetate were found to be the most important contributors to obtaining maximum functionalization/carboxylation. By examining contour plots and interaction plots, it was determined that optimal functionalization is obtained in a temperature range of $115-120^{\circ} \mathrm{C}$ with a reaction time of $54 \mathrm{~h}$ using a mixture of $6 \mathrm{~mL} \mathrm{DMF}, 200 \mathrm{mg}$ INTs, $800 \mathrm{mg}$ 2-bromoacetic acid and $60 \mathrm{mg}$ silver acetate.
\end{abstract}

Reprinted from Inorganics. Cite as: Raichman, D.; Strawser, D.; Lellouche, J.-P. Design of Experiments: Optimizing the Polycarboxylation/Functionalization of Tungsten Disulfide Nanotubes. Inorganics 2014, 2, 455-467.

\section{Introduction}

Design of experiments (DOE) [1,2] is a widely used discipline applied in a variety of areas, including engineering [3-5], social sciences [6] and natural sciences [7-9]. Design of experiments is a powerful statistical methodology in its own right, with a number of software applications readily available to aid researchers in both designing and globally optimizing multi-parametric experiments to achieve the best results through analysis and interpretation. In this context, combining software-based applications with a researcher's experience and scientific intuition is a powerfully growing trend that typically results in significant savings in time and materials. The design of experiments methodology includes formal, planned experimentation with the goal of optimizing a set of reaction parameters that may disclose synergism between reaction parameters. The optimization typically comprises six main steps: (1) selection of variables and defining their range of variation; (2) selection of responses; (3) experimental design selection; (4) performing the designed experiments in random order; (5) determination of coefficients in a mathematical model; and (6) predicting the response and evaluating the model relevance. In this context, we designed a series of experiments to optimize globally the reaction conditions for maximizing the yield of covalently functionalized tungsten disulfide inorganic fullerene-like nanotubes ( $\mathrm{WS}_{2}$ INTs). This specific functionalization reaction comprises a polycarboxylation technique $[10,11]$, developed recently in our laboratories, that uses a modified highly electrophilic Vilsmeier-Haack reagent [12]. 
It is well known that classical Vilsmeier-Haack reactions use DMF (secondary $N$-formyl amine) and $\mathrm{POCl}_{3} / \mathrm{SOCl}_{2}$ to effect the formylation of a wide range of electrophilic substrates via intermediate electrophilic iminium salts of Type A (Scheme 1a). Such Vilsmeier-Haack reactions have been studied extensively and found to be quite versatile, leading to a number of oxygen and nitrogen heterocycles [13-18], as well. For example, a modified Vilsmeier-Haack reagent that uses ethyl chloroformate in place of $\mathrm{POCl}_{3}$ was found useful when reacted with active methylene compounds [19]. Analogously, we have discovered that by using a mixture containing DMF (a 2nd $\mathrm{N}$-CHO amine) and O-alkylating 2-bromoacetic acid with catalysis by $\mathrm{Ag}(\mathrm{I}) \mathrm{OAc}, \mathrm{WS}_{2}$ nanotubes may be polycarboxylated readily and quite effectively according to the mechanism described in Scheme 1c. Indeed, DMF removal or replacement with other polar, non-protic materials/solvents (e.g., 1,4-dioxane, DME, etc.) leads to unsuccessful polycarboxylation. This strict requirement of DMF (2nd $\mathrm{N}$-CHO amine) as an essential component of the reaction mixture led us to propose and detail a corresponding Vilsmeier-Haack-like reaction mechanism, displayed in Scheme 1c.

Interestingly, silver acetate $(\mathrm{Ag}(\mathrm{I}) \mathrm{OAc})$ was also included as an essential reaction factor due to its well-known ability to chemically trap halogens and, thereby, assist in halogen (Br) abstraction. Depicted in Scheme $1 \mathrm{~b}$ is the reaction of $\mathrm{Ag}(\mathrm{I}) \mathrm{OAc}$ with the $\mathrm{Br}$ atom of bromoacetic acid, leading to the formation of the Vilsmeier-Haack complex of Type B1 with subsequent precipitation of $\mathrm{Ag}(\mathrm{I}) \mathrm{Br}$.

Because several reaction factors are involved in the polycarboxylation reaction mentioned above, we selected a DOE methodology as the most economical means of optimizing the reaction factors to produce the highest yields of polycarboxylation. In this context, the level of polycarboxylation was determined indirectly by reacting the functionalized INTs with an excess of 1,3-diaminopropane, resulting in a terminal primary amine that was quantified by the Kaiser test [20]. Due to a one-to-one reaction between the diamine and carboxylic acid, the amount of terminal amine is equal to the amount of carboxylic acid.

In addition to the Kaiser test; further confirmation of the successful functionalization of the $\mathrm{WS}_{2}$ INTs was obtained by a combination of FT-IR; TGA and zeta potential analyses (see the Supplementary Information).

Thus far, we have investigated this unique functionalization method only for the polycarboxylation of $\mathrm{WS}_{2}$ nanotubes. However, we strongly believe it may prove applicable for similar polycarboxylation of other layered dichalcogenide nanomaterials $\left(M \mathrm{~S}(\mathrm{Se})_{2}, M=\mathrm{Mo}, \mathrm{Sn}\right)$. 
Scheme 1. (a) Generalized Vilsmeier-Haack generation of electrophilic iminium salts; (b) Vilsmeier-Haack generation of DMF-based electrophilic iminium salt complex with bromophilic $\operatorname{Ag}(\mathrm{I})$ assistance in halide abstraction; (c) sulfur-mediated nucleophilic addition of the iminium salt complex to the outermost sulfur atoms of $\mathrm{WS}_{2}$ INTs, producing the corresponding polycarboxylated INTs.

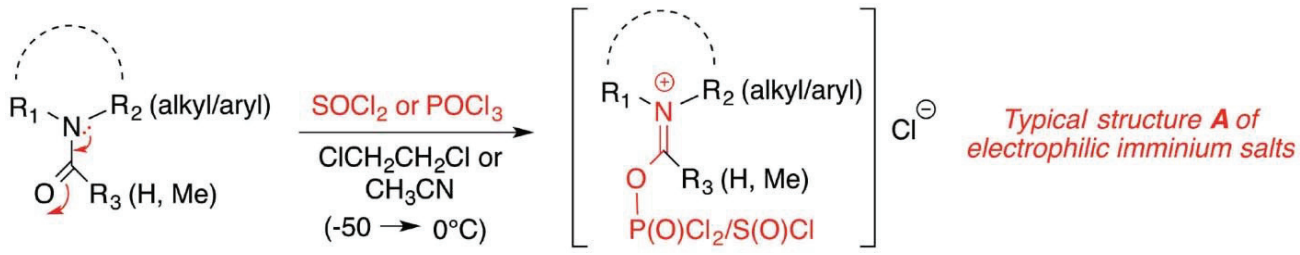

(a)

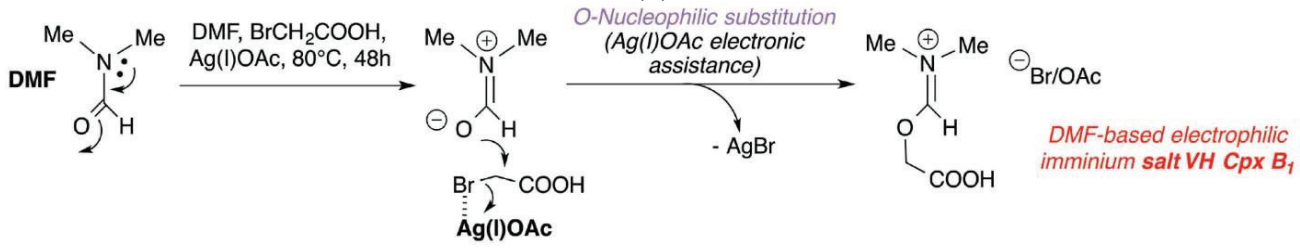

(b)

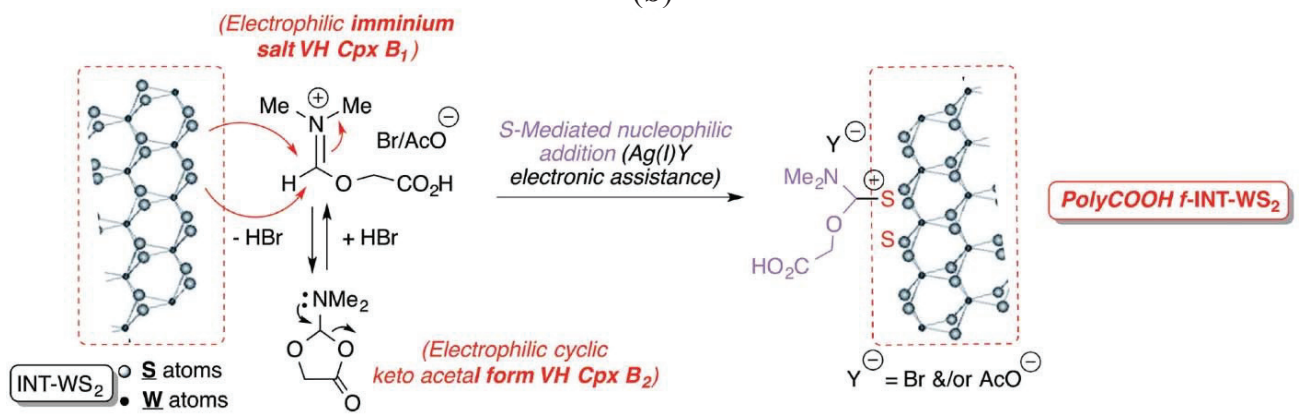

(c)

\section{Results and Discussion}

Because the level of surface functionalization of the $\mathrm{WS}_{2}$ INTs by the Vilsmeier-Haack-like reaction is of critical importance in determining the coordination capability of the corresponding optimally surface-engineered $\mathrm{WS}_{2}$ INTs, a statistically designed experiment was implemented using the design of experiment (DOE) methodology. The goal of the design was to disclose an optimal set of reaction conditions that would result in the maximized level of surface functionalization of the $\mathrm{WS}_{2}$ INTs. This DOE study enables varying more than one factor/reaction condition at a time for process optimization, even when several influential factors are involved. In addition, this multi-factor approach not only enables running fewer experiments, but enables the study of the interactions between the reaction factors and how these interactions influence the final result. These advantages are unavailable with the more commonly used one factor at a time (OFAT) optimization methods. 
Based on our current process knowledge, four factors (reaction parameters) likely to affect significantly the functionalization process were identified: DMF, silver acetate $(\mathrm{Ag}(\mathrm{I}) \mathrm{OAc}), \mathrm{WS}_{2}$ INTs and 2-bromoacetic acid $\left(\mathrm{BrCH}_{2} \mathrm{COOH}\right)$. In addition, two other factors, time and temperature, were included in the design. The experimental design and subsequent analysis of the significance of each factor and associated interactive effects were performed using a two-level factorial statistical design in conjunction with statistical software $\left(\right.$ MiniTab $\left.^{\circledR} 16\right)$ based on quadratic programming. Pareto analysis (Figure 1) was used to disclose which reaction parameters are process active. Using the software default value for $\alpha$ of 0.05 , all absolute magnitude effect values fall below the software error-calculated reference line for statistical significance (vertical red line, value 0.3361 ), indicating that none of the factors nor interactive effects are statistically significant. However, the software analysis indicates that time, temperature and the amount of silver acetate are the three most important contributors to obtaining maximum functionalization, and these warrant attention in the subsequent analyses. From the prediction model that was obtained, an optimal functionalization yield was obtained (1.21 mmol COOH groups/g) at an optimal temperature of $120^{\circ} \mathrm{C}$ and a reaction time of $54 \mathrm{~h}$.

Figure 1. Pareto chart of the effects of the factors on the amount of functionalization obtained. Values to the left of the reference line (vertical red line) are not statistically significant.

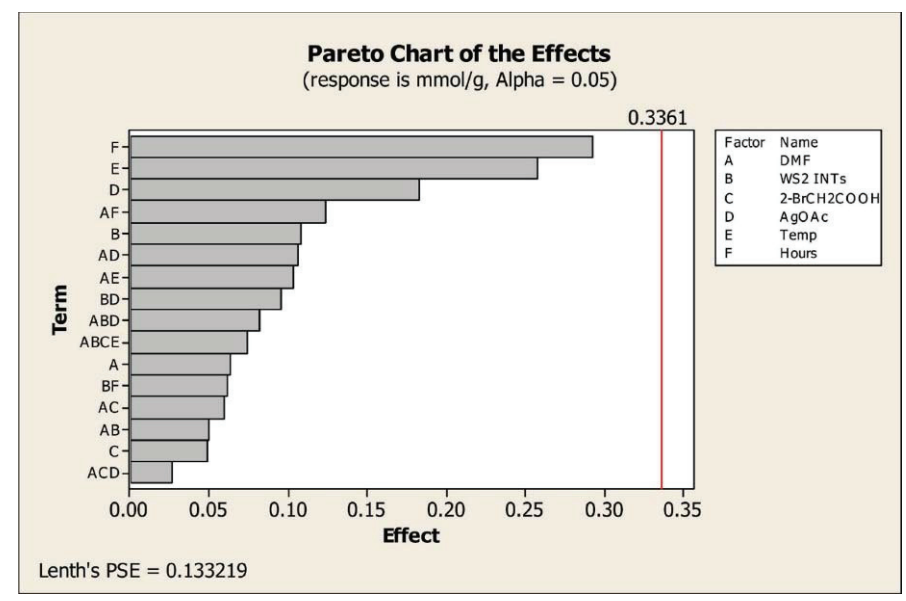

Figure 2 displays the effect of the two factors, temperature and time, on the amount of functionalization obtained per gram of INTs. Clearly, a higher temperature $\left(120^{\circ} \mathrm{C}\right)$ for a longer time $(54 \mathrm{~h})$ results in the highest level of functionalization.

To assess the effect of each of the other factors together with temperature on the amount of functionalization obtained when the reaction is conducted for $54 \mathrm{~h}$, a series of contour plots was made. For several of the factors, two combinations of temperature and factor $\left(\mathrm{WS}_{2} \mathrm{INTs}_{\mathrm{N}}\right.$ $\mathrm{BrCH}_{2} \mathrm{COOH}$, DMF or AgOAc) concentration produce the highest amount of carboxylation. When a higher temperature is used, the factor under evaluation does not affect the yield of carboxylation. However, in a lower temperature range, the highest amount of carboxylation is achieved only when the factor concentration is within a specific range. 
Figure 2. Amount (mmol) of carboxylic acid functional groups found per gram of $\mathrm{WS}_{2}$ INTs. Results are grouped by temperature and time. A higher temperature and a longer time increase the level of functionalization.
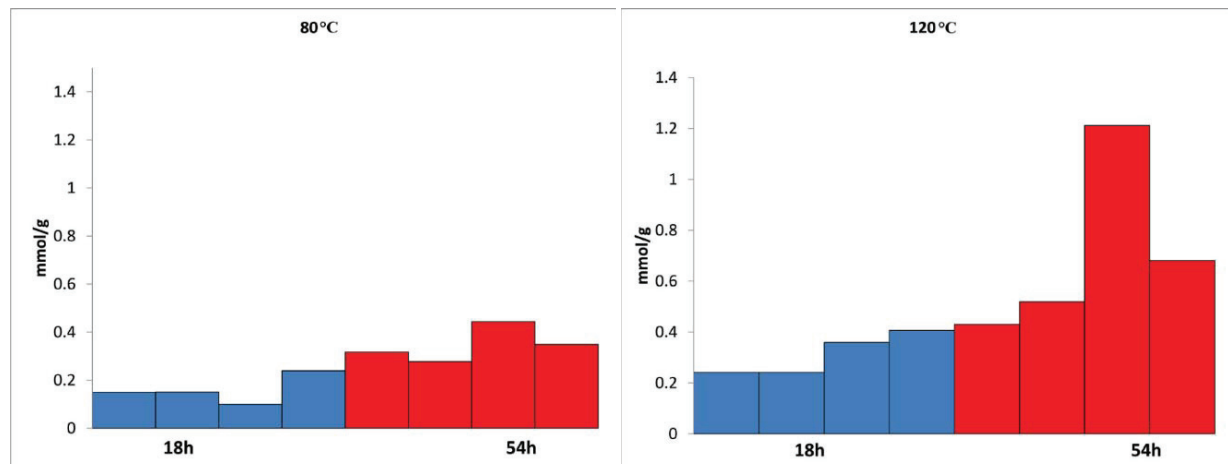

Figure 3 displays the effect of the dual factors, temperature $\left({ }^{\circ} \mathrm{C}\right)$ and quantity $(\mathrm{mg})$ of INTs, on the reaction mixture.

Figure 3. Contour plot of the amount (mmol) of carboxylation per gram of INTs obtained as the dual factors, temperature and amount of INTs in the reaction mixture, are varied at a constant reaction time of $54 \mathrm{~h}$.

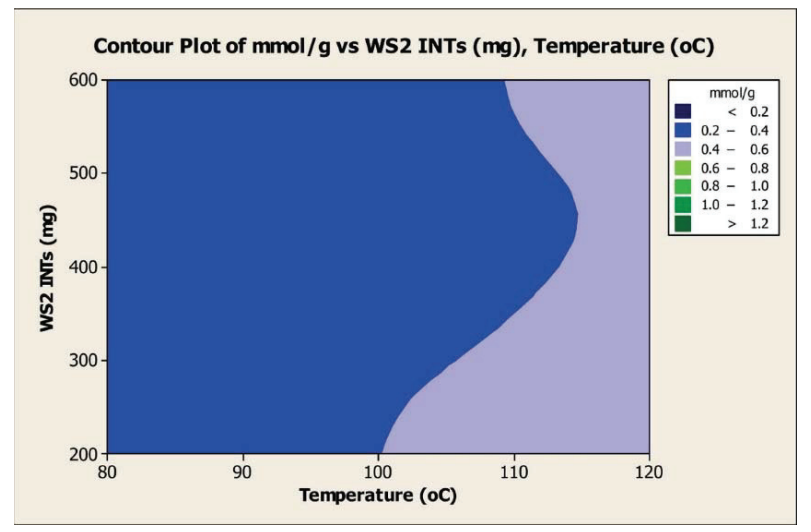

Two areas are revealed in which maximum carboxylation $(0.4-0.6 \mathrm{mmol} / \mathrm{g})$ can be obtained at a 54-h reaction time; by using 200-300 mg of INTs in the mixture with the temperature maintained in the range of $105-120{ }^{\circ} \mathrm{C}$ and using $200-600 \mathrm{mg}$ INTs with a temperature range of $115-120{ }^{\circ} \mathrm{C}$. Interestingly, a slightly lower amount of carboxylation $(0.2-0.4 \mathrm{mmol} / \mathrm{g})$ can be obtained using an INT level in the range of $200-600 \mathrm{mg}$ with a decrease of temperature in a range of $80-100{ }^{\circ} \mathrm{C}$. This suggests that it may be possible to customize the yield of carboxylation by tuning the amount of INTs in the reaction mixture together with the temperature.

Figure 4 displays the effect of the dual factors, temperature $\left({ }^{\circ} \mathrm{C}\right)$ and quantity $(\mathrm{mg}, \mathrm{mmol})$ of 2-bromoacetic acid in the reaction mixture. Two areas are revealed in which maximum carboxylation 
(0.4-0.6 $\mathrm{mmol} / \mathrm{g})$ can be obtained at a 54-h reaction time; by using 800-1100 $\mathrm{mg}(5.76-7.92 \mathrm{mmol})$ of 2-bromoacetic acid in the mixture with temperature maintained in a range of $105-120^{\circ} \mathrm{C}$ and using 800-2250 mg (5.76-16.2 mmol) of 2-bromoacetic acid with a temperature range of $115-120^{\circ} \mathrm{C}$. As mentioned above, with the INT concentration, by tuning the amount of 2-bromoacetic acid (800-2250 mg, 5.76-16.2 mmol) and the temperature $\left(80-100{ }^{\circ} \mathrm{C}\right)$, it may be possible to produce a controlled level of carboxylation within the range of $0.2-0.4 \mathrm{mmol} / \mathrm{g}$.

Figure 4. Contour plot of the amount (mmol) of carboxylation per gram of INTs obtained as the dual factors, temperature and amount of 2-bromoacetic acid in the reaction mixture, are varied at a constant reaction time of $54 \mathrm{~h}$.

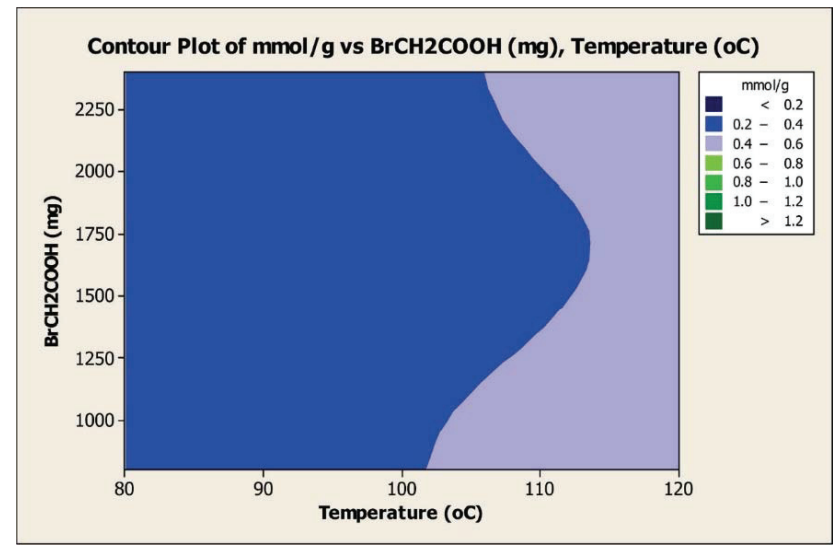

Figure 5 displays the effect of the dual factors, temperature $\left({ }^{\circ} \mathrm{C}\right)$ and quantity $(\mathrm{mL})$ of DMF in the reaction mixture. Two areas are revealed in which maximum carboxylation $(0.4-0.6 \mathrm{mmol} / \mathrm{g})$ can be obtained at a 54-h reaction time; by using $2-2.5 \mathrm{~mL}$ of DMF in the mixture with temperature maintained in a range of $110-120{ }^{\circ} \mathrm{C}$, and using 2-6 $\mathrm{mL}$ DMF with a temperature range of $115-120^{\circ} \mathrm{C}$. As mentioned above, for both INT and the 2-bromoacetic acid concentration, by tuning the amount of DMF $(2-6 \mathrm{~mL})$ and temperature $\left(80-100^{\circ} \mathrm{C}\right)$, it may be possible to produce a controlled level of carboxylation within the range of $0.2-0.4 \mathrm{mmol} / \mathrm{g}$.

Figure 6 displays the effect of the dual factors, temperature $\left({ }^{\circ} \mathrm{C}\right)$ and quantity $(\mathrm{mg})$ of $\mathrm{AgOAc}$ in the reaction mixture. Three zones of carboxylation yield are clearly identified, indicating that several sets of AgOAc concentration and temperature can be used to achieve the selected level of carboxylation. For example, maximum carboxylation $(0.6-0.8 \mathrm{mmol} / \mathrm{g})$ can be obtained at a $54-\mathrm{h}$ reaction time by using AgOAc in a range of 55-60 $\mathrm{mg}(0.33-0.36 \mathrm{mmol})$ in the mixture with temperature maintained in a range of $115-120^{\circ} \mathrm{C}$. The following combinations of AgOAc/temperature produce a lower carboxylation level of $0.4-0.6 \mathrm{mmol} / \mathrm{g}: 60 \mathrm{mg}(0.36 \mathrm{mmol}) / 100-110{ }^{\circ} \mathrm{C}$; $50-60 \mathrm{mg}(0.30-0.36 \mathrm{mmol}) / 105-110{ }^{\circ} \mathrm{C} ; 40-50 \mathrm{mg}(0.24-0.30 \mathrm{mmol}) / 115-120{ }^{\circ} \mathrm{C}$. In addition, a carboxylation level of $0.2-0.4$ can be achieved over the entire temperature range of $80-120^{\circ} \mathrm{C}$ if the AgOAc level is maintained within $20-35 \mathrm{mg}(0.12-0.21 \mathrm{mmol})$. Similarly, this lower carboxylation level is achievable over the entire range of $20-60 \mathrm{mg}(0.12-0.36 \mathrm{mmol}) \mathrm{AgOAc}$ by maintaining a lower temperature range of $80-100{ }^{\circ} \mathrm{C}$. 
Figure 5. Contour plot of the amount (mmol) of carboxylation per gram of INTs obtained as the dual factors, temperature and amount of DMF in the reaction mixture, are varied at a constant reaction time of $54 \mathrm{~h}$.

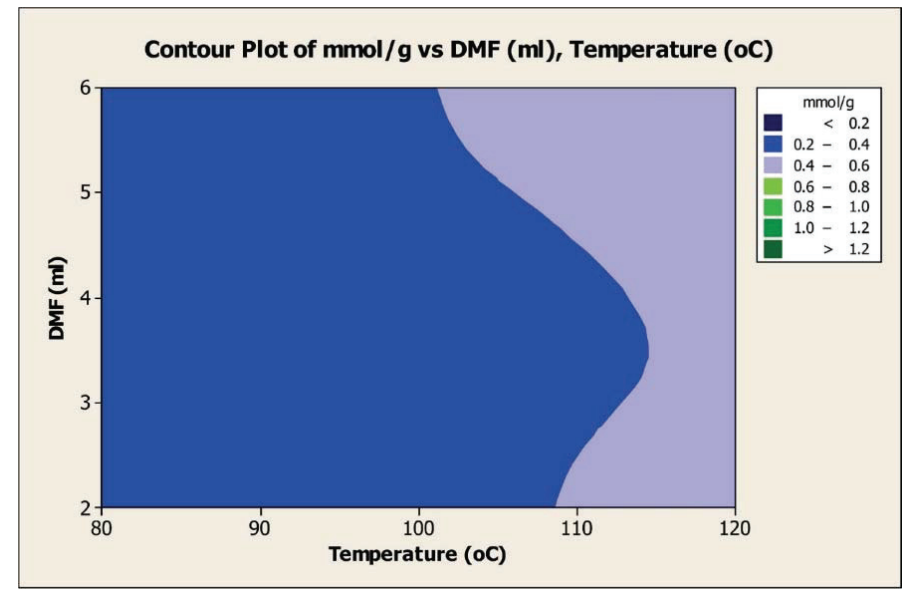

Figure 6. Contour plot of the amount (mmol) of carboxylation per gram of INTs obtained as the dual factors, temperature and amount of AgOAc in the reaction mixture, are varied at a constant reaction time of $54 \mathrm{~h}$.

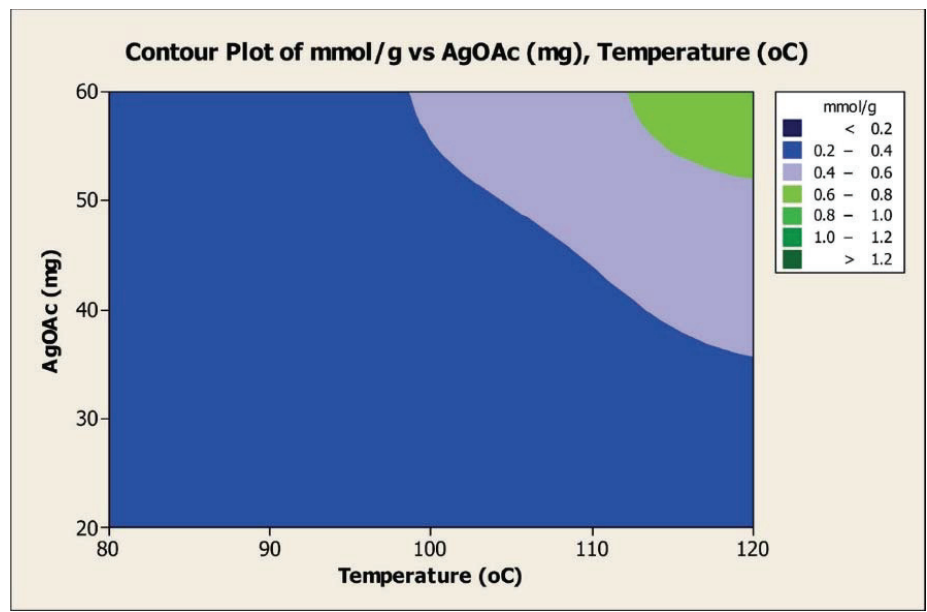

Figure 7 displays the effect on carboxylation yield with the dual factors, temperature $\left({ }^{\circ} \mathrm{C}\right)$ and time (h). For this factor combination, four zones of carboxylation yield are identified. As noted with the factors temperature and AgOAc, several sets of the two factors can be used to achieve a selected level of carboxylation. As examples for achieving the highest carboxylation level $(0.6-0.8 \mathrm{mmol} / \mathrm{g})$, a time range of $45-60 \mathrm{~h}$ at a temperature of $120^{\circ} \mathrm{C}$ or a temperature in a range of $110-120^{\circ} \mathrm{C}$ for $54 \mathrm{~h}$ can be used. Similar analyses can be done to find ranges of temperature and time to produce the lower carboxylation levels displayed in Figure 7. 
Figure 7. Contour plot of the amount (mmol) of carboxylation per gram of INTs obtained as the dual factors, temperature $\left({ }^{\circ} \mathrm{C}\right)$ and time $(\mathrm{h})$, are varied.

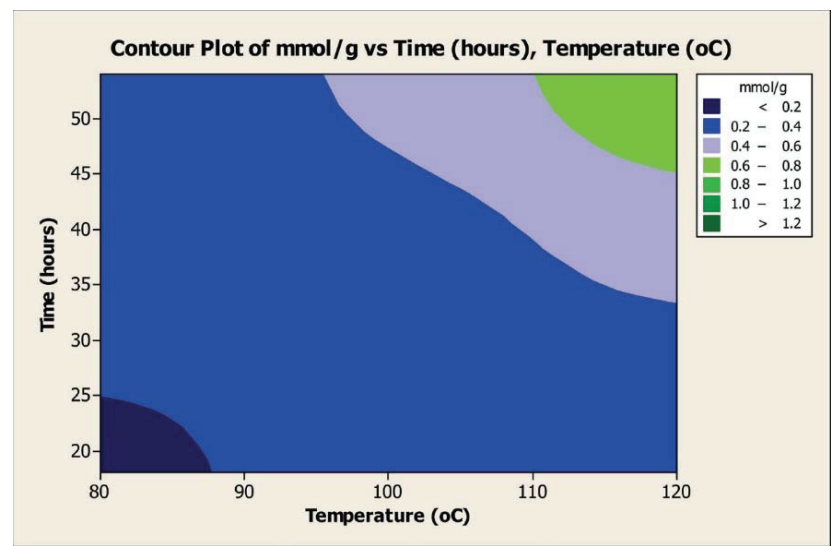

Analysis of the contour plots for a 54-h reaction time (Figures 3-7) indicates that only the temperature range of $115-120{ }^{\circ} \mathrm{C}$ is common to all of the factors to produce the maximum degree of carboxylation. Using this temperature range, the levels of each factor to achieve maximum carboxylation are as follows. INTs 200-600 mg; 2-bromoacetic acid 800-2250 mg (5.76-16.2 mmol); DMF 2-6 mL and AgOAc 55-60 mg (0.33-0.36 mmol).

Figure 8. Interaction plot of the effects of the factors on the degree of carboxylation at a reaction time of $54 \mathrm{~h}$.

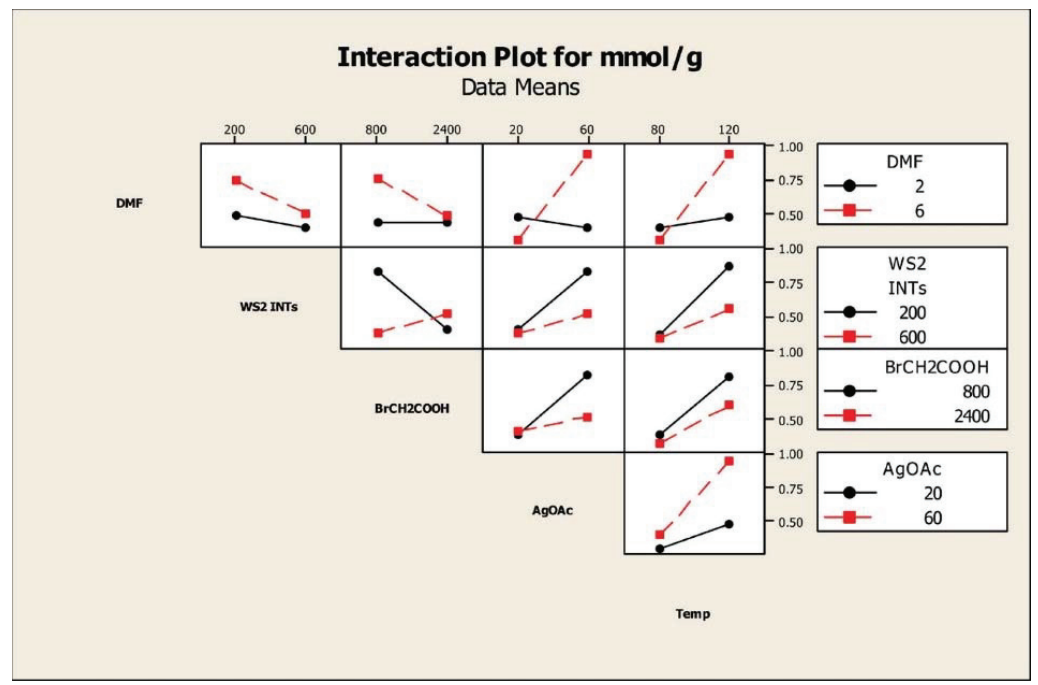

The influence of the factors on the degree of carboxylation was examined by interaction plots. Separate interaction plots were made for reaction times of $18 \mathrm{~h}$ and $54 \mathrm{~h}$. Figure 8 displays the interaction plot for a 54-h reaction time. The temperature column indicates that a reaction 
temperature of $80{ }^{\circ} \mathrm{C}$ results in not only a much lower amount of carboxylation than a reaction temperature of $120^{\circ} \mathrm{C}$, but, at the levels tested, changing the concentrations of the four factors, DMF, INT, 2-bromoacetic acid and AgOAc, has little effect on the degree of carboxylation. In contrast, at $120{ }^{\circ} \mathrm{C}$, higher concentrations of DMF $(6 \mathrm{~mL})$ and $\mathrm{AgOAc}(60 \mathrm{mg}, 0.36 \mathrm{mmol})$ with lower concentrations of INTs (200 mg) and 2-bromoacetic acid $(800 \mathrm{mg}, 5.76 \mathrm{mmol})$ produce higher degrees of carboxylation. Note that this analysis is in agreement with the findings of the contour plots (Figures 3-7).

The AgOAc column shows similar effects, with the lower concentration of AgOAc producing a lower amount of carboxylation than a higher concentration, and changing the concentration of the other factors has little effect on the degree of carboxylation. When a higher concentration of $\mathrm{AgOAc}$ is used, the degree of carboxylation is increased with a higher DMF concentration, but with lower concentrations of INTs and 2-bromoacetic acid. The 2-bromoacetic acid column indicates that a higher degree of carboxylation is obtained using a lower concentration of acid along with a lower concentration of INTs and a higher concentration of DMF. Similarly, the $\mathrm{WS}_{2}$ INT column indicates that using a lower concentration of INTs and a higher concentration of DMF produces a higher degree of carboxylation.

Figure 9 displays the interaction plot for an 18-h reaction time.

Figure 9. Interaction plot of the effects of the factors on the degree of carboxylation at a reaction time of $18 \mathrm{~h}$.

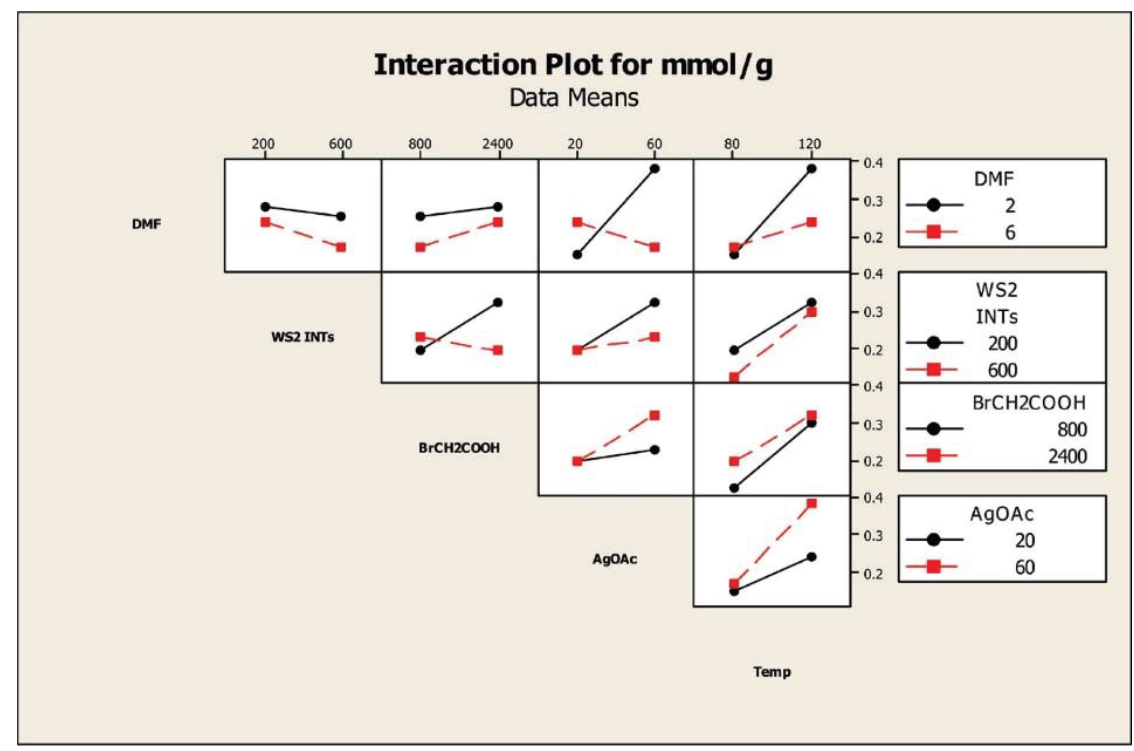

The temperature column indicates that a reaction time of $18 \mathrm{~h}$ with optimized factors of $120{ }^{\circ} \mathrm{C}$, $2 \mathrm{~mL}$ DMF, $60 \mathrm{mg}$ (0.36 mmol) AgOAc, 200-600 mg INTs, and 800-2400 mg (5.76-17.3 mmol) 2-bromoacetic acid gives a maximum amount of carboxylation that is less than half the amount that is obtained with optimized factors when the reaction time is $54 \mathrm{~h}$. 


\section{Experimental Section}

\subsection{Materials}

Tungsten disulfide nanotubes (WS 2 -INTs) were provided by NanoMaterials Ltd. (Yavne, Israel). All reagents and solvents were purchased from commercial sources and used without further purification.

\subsection{Methods}

Carboxylated $\mathrm{WS}_{2}$ INTs

The carboxylated INTs were prepared using the conditions in Table 1. Details for Sample 1 are presented as a representative example.

Table 1. Sample parameters for the carboxylation reaction.

\begin{tabular}{cccccccc}
\hline Sample & $\begin{array}{c}\text { DMF } \\
\mathbf{m L}\end{array}$ & $\begin{array}{c}\mathbf{W S}_{\mathbf{2}} \text { INTs } \\
\mathbf{m g}\end{array}$ & $\begin{array}{c}\text { 2-Bromoacetic acid } \\
\mathbf{m g}\end{array}$ & $\begin{array}{c}\text { AgOAc } \\
\mathbf{m g}\end{array}$ & $\begin{array}{c}\text { Temp } \\
{ }^{\circ} \mathbf{C}\end{array}$ & $\begin{array}{c}\text { Time } \\
\mathbf{h}\end{array}$ & $\begin{array}{c}\text { Carboxylation } \\
\mathbf{m m o l} / \mathbf{g}\end{array}$ \\
\hline 1 & 2 & 200 & 800 & 20 & 80 & 18 & 0.150 \\
2 & 6 & 200 & 800 & 20 & 120 & 18 & 0.241 \\
3 & 2 & 600 & 800 & 20 & 120 & 54 & 0.430 \\
4 & 6 & 600 & 800 & 20 & 80 & 54 & 0.318 \\
5 & 2 & 200 & 2400 & 20 & 120 & 54 & 0.519 \\
6 & 6 & 200 & 2400 & 20 & 80 & 54 & 0.278 \\
7 & 2 & 600 & 2400 & 20 & 80 & 18 & 0.151 \\
8 & 6 & 600 & 2400 & 20 & 120 & 18 & 0.241 \\
9 & 2 & 200 & 800 & 60 & 80 & 54 & 0.444 \\
10 & 6 & 200 & 800 & 60 & 120 & 54 & 1.212 \\
11 & 2 & 600 & 800 & 60 & 120 & 18 & 0.359 \\
12 & 6 & 600 & 800 & 60 & 80 & 18 & 0.100 \\
13 & 2 & 200 & 2400 & 60 & 120 & 18 & 0.406 \\
14 & 6 & 200 & 2400 & 60 & 80 & 18 & 0.240 \\
15 & 2 & 600 & 2400 & 60 & 80 & 54 & 0.349 \\
16 & 6 & 600 & 2400 & 60 & 120 & 54 & 0.681 \\
17 & 4 & 400 & 1600 & 40 & 100 & 36 & 0.346 \\
\hline
\end{tabular}

To a solution of 2-bromoacetic acid $(0.80 \mathrm{~g}, 5.76 \mathrm{mmol})$ in DMF $(2 \mathrm{~mL})$ was added $\mathrm{AgOAc}$ (20 mg, $0.12 \mathrm{mmol}$ ) and $\mathrm{WS}_{2}$ INTs $\left(200 \mathrm{mg}\right.$ ). The mixture was heated in an oil bath to $80{ }^{\circ} \mathrm{C}$ and stirred for $18 \mathrm{~h}$. After cooling to room temperature, the mixture was centrifuged and the supernatant discarded. The solids were washed with ethanol followed by centrifugation $(11,000 \mathrm{rpm}$ $5 \mathrm{~min}) 5$ times and dried under vacuum to obtain carboxylated $\mathrm{WS}_{2}$ INTs containing $0.150 \mathrm{mmol}$ carboxylic acid per gram of INT.

Quantification of the carboxylic acid was done indirectly by coupling 1,3-diaminopropane to the carboxylic acid using EDC as a coupling agent, followed by the Kaiser test for terminal amines $[10,13]$. 


\section{Conclusions}

By using the design of experiments (DOE) methodology, six reaction factors were identified and optimized to maximize the degree of functionalization of inorganic $\mathrm{WS}_{2}$ INTs with a carboxylic acid shell via a modified highly electrophilic Vilsmeier-Haack reaction. Initial studies produced functionalized INTs with $0.5 \mathrm{mmol} \mathrm{COOH}$ groups per gram of INT as quantified by the Kaiser test. Moreover, a statistically relevant DOE global optimization resulted in functionalization increasing by a factor of 2.4 to $1.2 \mathrm{mmol}$ of accessible $\mathrm{COOH}$ groups per gram of chemically-modified INTs. Time, temperature and amount of silver acetate $(\mathrm{Ag}(\mathrm{I}) \mathrm{OAc})$ were found to be the most important factors that affect the functionalization yield.

In addition, contour plots of interacting reaction parameters suggest that it may be possible to control the amount of polyCOOH functionalization by tuning the amounts of INTs, 2-bromoacetic acid, DMF and $\mathrm{Ag}(\mathrm{I}) \mathrm{OAc}$ in the reaction mixture in conjunction with reaction temperature. This attractive capability may prove valuable for optimizing the use of functionalized $\mathrm{WS}_{2} \mathrm{INTs}_{\mathrm{N}}$ in important applications, such as functional nanoscale fillers for the mechanical reinforcement of polymeric matrices with optimal interfacial phase interactions.

\section{Supplementary Information}

\section{FT-IR}

FT-IR spectra were recorded on an FT-IR Tensor 27 spectrometer (Bruker) using ATR.

Figure S1 displays the FT-IR spectrum of carboxylated WS2 INTs with peaks of interest marked. The peaks can be assigned as follows (wavenumbers $v$ in $\mathrm{cm}^{-1}$ ). 3367, O-H stretch; 2881, C-H stretch; $1747, \mathrm{C}=\mathrm{O}$ stretch of carboxylic acid; $1467, \mathrm{C}-\mathrm{H}$ bend; 1360, $\mathrm{C}-\mathrm{H}$ rock; $1039, \mathrm{C}=\mathrm{S}$ stretch (indicative of covalent attachment to sulfur of the INTs); 946, O-H of carboxylic acid.

Figure S1. FT-IR spectrum of carboxylated $\mathrm{WS}_{2}$ INTs.

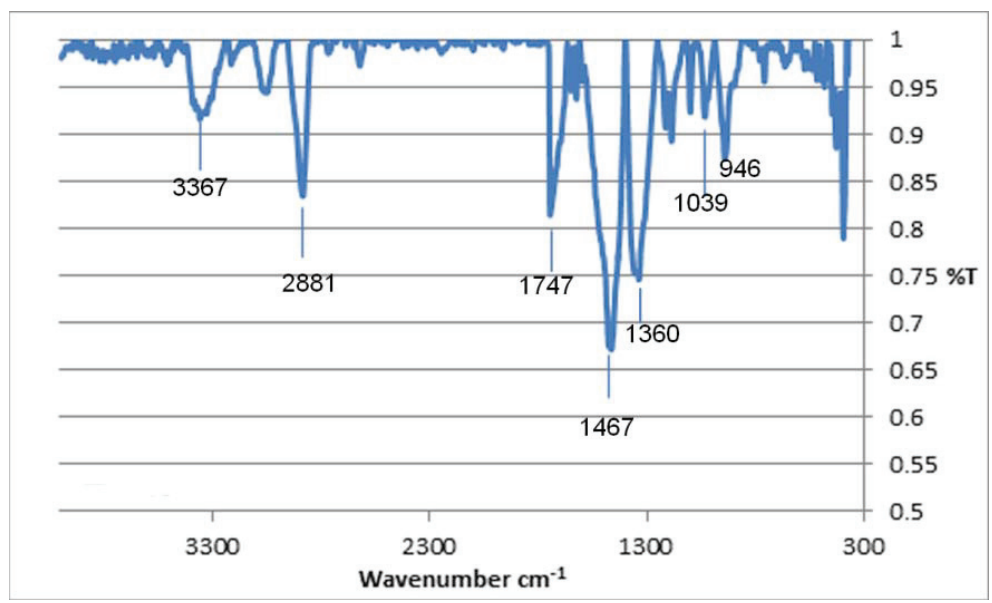




\section{Thermogravimetric Analysis}

Thermogravimetric analysis was performed on a TA Q600-0348, model SDT Q600 (Thermofinnigan) using a temperature profile of $25-800{ }^{\circ} \mathrm{C}$ at $10{ }^{\circ} \mathrm{C} / \mathrm{min}$ under $\mathrm{N}_{2}$ flow $(10 \mathrm{~mL} / \mathrm{min})$ with sample masses ranging from 5-12 mg.

The TGA analysis of the inorganic nanotubes before and after carboxylation is displayed in Figure S2. Weight losses were $1.39 \%$ and $17.12 \%$ for the untreated and carboxylated $\mathrm{WS}_{2}$ INTs respectively, indicating that organic material is attached to the surface of the treated nanotubes.

Figure S2. TGA plots of $\mathrm{WS}_{2}$ INTs.

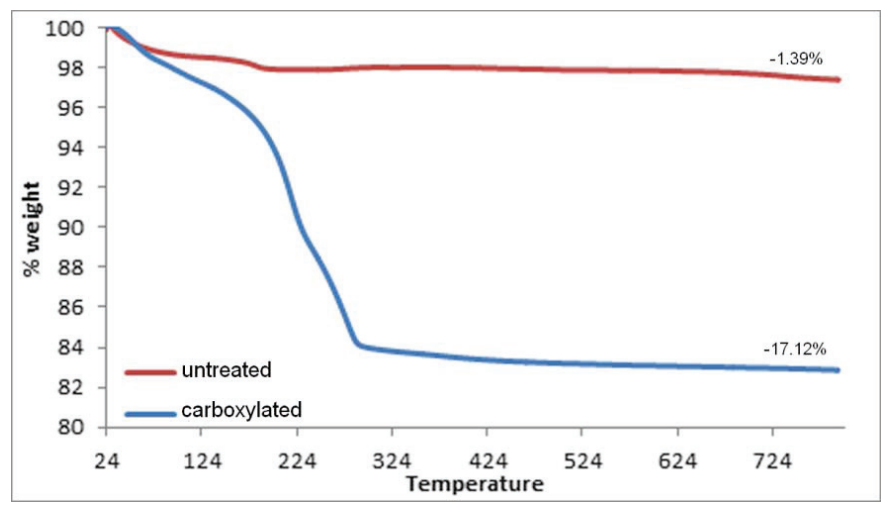

\section{Zeta Potential}

Nanoparticle surface charge ( $\xi$ potential) was determined using a Zetasizer Nano-ZS (Malvern Instruments Ltd. Worcestershire, UK) in water at $25^{\circ} \mathrm{C}$ and $150 \mathrm{~V}$. Dispersions were prepared with an ElmaSonic S30 sonicator (Elma GmbH \& Co., Singen, DE). $5 \mathrm{mg}$ of $\mathrm{WS}_{2}$ INTs or carboxylated $\mathrm{WS}_{2}$ INTs were dispersed in $15 \mathrm{ml}$ ultra-pure $\mathrm{H}_{2} \mathrm{O}$ (resitivity $>18 \mathrm{Mohm} \bullet \mathrm{cm}$ ) by sonication for one minute. The Zeta potential was measured immediately after sonication. The analysis showed -27.8 $\mathrm{mV}$ and $-17.3 \mathrm{mV}$ for the carboxylated and untreated $\mathrm{WS}_{2}$ INTs, respectively.

\section{Acknowledgments}

We thank NanoMaterials Ltd. for their generous gift of $\mathrm{WS}_{2}$ nanotubes and the Israel National Nanotechnology Initiative Focal Technology Area proposal Inorganic Nanotubes: From Nanomechanics to Improved Nanocomposites, (Reshef Tenne, program coordinator) for partial funding of this research.

\section{Author Contributions}

Daniel Raichman performed most of the experimental work, prepared drafts of the experimental section, prepared the initial images and researched the design of experiments. David Strawser assisted with experimental procedures and design of experiments processing, finalized images and edited much 
of the manuscript. Jean-Paul Lellouche initiated this $\mathrm{WS}_{2}$ INTs functionalization project, provided full research assistance in the selection of the influential factors, checked the entire manuscript and provided all of the necessary budget, laboratory facilities and intellectual support for conducting the corresponding research work.

\section{Conflicts of Interest}

The authors declare no conflict of interest.

\section{References}

1. Montgomery, D.C. Design and Analysis of Experiments, 6th ed.; Wiley: New York, NY, USA, 2005.

2. Engineering Statistics Handbook. Available online: http://www.itl.nist.gov/div898/ handbook/pri/section1/pri11.htm (accessed on 20 February 2014).

3. Castillo, P.; Magnin, J.P.; Velasquez, M.; Willisom, J. Modeling and Optimization of Hydrogen Production by the Methodology of Design of Experiments (DOE): Interaction between Lactate Concentration and Light Luminosity. Energy Procedia 2012, 29, 357-366.

4. Imandi, S.B.; Bandaru, V.V.R.; Somalanka, S.R.; Bandaru, S.R.; Garapati, H.R. Application of Statistical Experimental Designs for the Optimization of Medium Constituents for the Production of Citric Acid from Pineapple Waste. Bioresour. Technol. 2009, 99, 4445-4450.

5. Varrone, C.; Giussani, B.; Izzo, G.; Massini, G.; Marone, A.; Signorini, A.; Wang, A. Statistical Optimization of Biohydrogen and Ethanol Production from Crude Glycerol by Microbial Mixed Culture. Int. J. Hydrog. Energy 2013, 37, 16479-16488.

6. Schneider, S. Experimental Design in the Behavioral and Social Sciences; SAGE Publications: Thousand Oaks, CA, USA, 2013.

7. Mandenius, C.K.; Brundin, A. Bioprocess Optimization Using Design-of-Experiments Methodology. Biotechnol. Prog. 2008, 24, 1191-1203.

8. Yates, F. Imperial Bureau of Soil Science Technical Communication No. 35: The Design and Analysis of Factorial Experiments; Imperial Bureau of Soil Science: London, UK, 1937.

9. Pilat, P.; Votruba, J.; Dobersky, P.; Prokop, A. Application of Mathematical Optimization Methods in Microbiology. Folia Microbiol. 1976, 21, 391-405.

10. Raichman, D.; Strawser, D.A.; Lellouche, J.P. Covalent Functionalization/Polycarboxylation of Tungsten Disulfide Inorganic Nanotubes. Nanoscale 2014, submitted for publication.

11. Lellouche, J.-P. Versatile chemical Modificaions of Hard Fullerene-Like Inorganic Tungsten Disulfide $\left(\mathrm{WS}_{2}\right)$ Nanotubes $\left(\mathrm{WS}_{2}\right.$ INTs). U.S. Provisional Application for Patent No. 61/865, 151,13 August 2013.

12. Vilsmeier, A.; Haack, A. Uber die Einwirkung von Halogen Phosphor auf Alkyl-formanilide. Ein neue Methode zur Darstellung sekundarer und tertiarer p-Alkylamino-benzaldehyde. Chem. Berichts 1927, 60, 119-122 (In German).

13. Sreenivasulu, M.; Rao, K.G.S. Vilsmeier reaction studies on some $\alpha, \beta$-unsaturated alkenones. Indian J. Chem. Sect. B 1989, 28, 594-595. 
14. Lilienkampf, A.; Johansson, M.P.; Wahala, K. (Z)-1-Aryl-1-haloalkenes as Intermediates in the Vilsmeier Haloformylation of Aryl Ketones. Org. Lett. 2003, 5, 3387-3390.

15. Ali, M.M.; Tasneem; Rajanna, K.C.; Saiprakash, P.K. An efficient and facile synthesis of 2-chloro-3-formyl quinolines frome acetanilides in micellar media by Vilsmeier-Haack cyclisation. Synlett 2001, 2, 251-253.

16. Quiroga, J.; Trilleras, J.; Galvez, J.; Insuasty, B.; Abonıa, R.; Nogueras, M.; Cobo, J.; Marchal, A. $\mathrm{C}$ - and $\mathrm{N}$-cyanoacetylation of 6 -aminopyrimidines with cyanoacetic acid and acetic anhydride. Tetrahedron Lett. 2008, 49, 5672-5675.

17. Giles, C.M.M.A.P.R. Synthesis Using Vilsmeier Reagents. New Directions in Organic and Biological Chemistry, 1st ed.; CRC Press: Boca Raton, FL, USA, 1994.

18. Rajanna, K.C.; Ali, M.M.; Solomon, F.; Saiprakash, P.K. Vilsmeier-Haack Formylation of Coumarin Derivatives. A Solvent Dependent Kinetic Study. Int. J. Chem. Kinet. 1996, 28, $865-872$.

19. Ikawa, K.; Takami, F.; Fukui, Y.; Takuyama, K. A Novel Reagent for the Vilsmeier-Haack Reaction. Tetrahedron Lett. 1969, 38, 3279-3281.

20. Kaiser, E.; Colescott, R.L.; Bossiriger, C.D.; Cook, P.I. Color Test for the Detection of Free Terminal Amino Groups in the Solid-phase Synthesis of Peptides. Anal. Biochem. 1970, 34, $595-598$. 


\title{
Noble-Metal Chalcogenide Nanotubes
}

\section{Nourdine Zibouche, Agnieszka Kuc, Pere Miró and Thomas Heine}

\begin{abstract}
We explore the stability and the electronic properties of hypothetical noble-metal chalcogenide nanotubes $\mathrm{PtS}_{2}, \mathrm{PtSe}_{2}, \mathrm{PdS}_{2}$ and $\mathrm{PdSe}_{2}$ by means of density functional theory calculations. Our findings show that the strain energy decreases inverse quadratically with the tube diameter, as is typical for other nanotubes. Moreover, the strain energy is independent of the tube chirality and converges towards the same value for large diameters. The band-structure calculations show that all noble-metal chalcogenide nanotubes are indirect band gap semiconductors. The corresponding band gaps increase with the nanotube diameter rapidly approaching the respective pristine 2D monolayer limit.
\end{abstract}

Reprinted from Inorganics. Cite as: Zibouche, N.; Kuc, A.; Miró, P.; Heine, T. Noble-Metal Chalcogenide Nanotubes. Inorganics 2014, 2, 556-564.

\section{Introduction}

Inorganic nanotubes (INTs) are a class of materials that are very attractive for many applications in nanotechnology due to their interesting physical and chemical properties, which arise from their low dimensionality. In 1930, Pauling had already stated that mismatched layered materials may form cylindrical shapes [1]. However, the first carbon-based tubular forms, namely carbon nanotubes (CNTs) have been observed by Iijima in 1991 [2]. At the same time, the synthesis of $\mathrm{WS}_{2}$ NTs by Tenne and co-workers [3], has demonstrated that these tubular systems are not limited to carbon, but can also be obtained from any other layered compound. Later on, several INTs have been prepared and produced such as transition-metal sulfides [3,4]. boron-nitrides [5,6], metal oxides [7,8], rare earth oxide [9] and more recently misfit layered compounds [10].

$\mathrm{WS}_{2}$ and $\mathrm{MoS}_{2}$ NTs, being the first synthesised INTs, are semiconductors. They have demonstrated excellent mechanical properties [11-16] and are known to be good solid lubricants [17]. They have also been suggested as scanning probe tips [18], catalysts [19], reinforcements for composite materials [20], photo-transistors [21], gas storage and host materials [22,23], etc. Later, other transition-metal chalcogenide (TMC) NTs have been reported such as $\mathrm{TiS}_{2}, \mathrm{NbS}_{2}, \mathrm{ReS}_{2}, \mathrm{TiSe}_{2}$ and $\mathrm{TaS}_{2}$ [24-28]. Subsequently, many more TMC NTs can be expected due to the large manifold of the layered TMC materials [29,30]. Nowadays, different techniques and strategies have been employed and developed for the synthesis and growth of large amount of NTs such as chemical transport technique [31], thermochemical decomposition [32] and in situ heating [33]. For example, $\mathrm{WS}_{2}$ and $\mathrm{MoS}_{2}$ NTs were produced using gas-solids reactions at high temperatures by the reduction of $\mathrm{WO}_{3}\left(\mathrm{MoO}_{3}\right)$ in the atmosphere of a mixture of $\mathrm{H}_{2}, \mathrm{~N}_{2}$ and $\mathrm{H}_{2} \mathrm{~S}$ gases $[3,4,34]$.

In this work, we aim to extend the scope of inorganic nanotubular materials by investigating noble-metal chalcogenide $M X_{2}$ single wall nanotubes, where $M=\mathrm{Pt}$, Pd and $X=\mathrm{S}$, Se. Tubular forms based on these materials have not yet been experimentally observed, however, considering that 
$\mathrm{PtS}_{2}, \mathrm{PtSe}_{2}, \mathrm{PdS}_{2}, \mathrm{PdSe}_{2}$ belong to the family of layered TMCs, one can expect that such compounds may also form nanotubes. Structure and electronic structure of these noble metal chalcogenides have been subject to controversial debate in the 1950s and 1960s. Two possible phases of the Pd- and Pt-based TMCs were suggested, namely orthorhombic (pyrite) and 1T. [35-38]. We have calculated the relative stability of bulk and monolayered forms of these TMCs. While the orthorhombic phase is preferable for the bulk $\mathrm{PdS}_{2}$ material (energy difference of $56 \mathrm{meV}$ - all energies are given per $M X_{2}$ formula unit), the monolayers (MLs) favor the $1 T$ arrangement (energy difference of 68 $\mathrm{meV}$ ). For the heavier $\mathrm{PdSe}_{2}$, we have found similar trends for the MLs, where the $1 T$ form is by $0.6 \mathrm{eV}$ more stable than the orthorhombic one. Bulk $\mathrm{PdSe}_{2}$ is unstable in the orthorhombic form, forbidding comparison with the $1 T$ structure. Therefore, the choice of the $1 T$ polytype to simulate the single-wall NTs is justified. In view of the renaissance of layered materials and the advance of experimental technology it is important to reexamine these phases and attempt exfoliation.

A recent study of bi- and monolayered noble-metal materials have shown interesting quantum confinement effects and electromechanical properties, suggesting them for applications in optoelectronics and flexible devices [39]. Therefore we have investigated, using density functional theory (DFT), the stability of the $M X_{2}$ NTs and their structural and electronic properties. The strain energy is found to be chirality independent and exhibits the characteristic dependence on the tube diameter $d\left(\sim 1 / d^{2}\right)$. The band structure analysis shows that noble-metal chalcogenide NTs are all semiconducting in a similar way to their ML counterparts. Unlike $\mathrm{MoS}_{2}$ and $\mathrm{WS}_{2} \mathrm{NTs}$, where the band gap is direct and indirect for zigzag and armchair, respectively, $\mathrm{PtX}_{2}$ and $\mathrm{PdX}_{2} \mathrm{NTs}$ have indirect band gaps which increase with the diameter.

\section{Computational Details}

All calculations were carried out using density functional theory with the PBE (Perdew-Burke-Ernzerhof) exchange-correlation functional [40] as implemented in the Crystal09 code [41]. We have used an all electron triple-zeta valence basis set with one polarization function for sulphur atoms [42], a scalar-relativistic pseudopotential with 18 valence electrons for platinum atoms [43], Hay and Wadt effective core potentials with small core for palladium atoms [41], and the relativistic multi-electron pseudopotential with six valence electrons for selenium atoms [44]. We have employed helical boundary conditions, as implemented in the Crystal09 code [41], for the generation of the NT structures in order to reduce computational costs. Lattice vectors and atomic positions of $M X_{2}$ MLs and NTs were fully optimized. The tube diameters considered here are in the range of $10-50 \AA$, corresponding to chiral indices $(10,0)-(32,0)$ and $(6,6)-(24,24)$ for zigzag and armchair NTs, respectively. The shrinking factors of 16 for MLs and 8 for NTs were used, resulting in 30 and $5 k$-points in the irreducible Brillouin zone, respectively, following the Monkhorst-Pack sampling [45]. Band structures were calculated along the high symmetry $k$-points using the $\mathrm{M}-\Gamma-\mathrm{K}-\mathrm{M}$ and $\mathrm{X}-\Gamma$ paths for MLs and NTs, respectively. 


\section{Results and Discussion}

The monolayered noble-metal chalcogenides, considered here as large diameter NT limits, were adopted in the $1 T$ polymorph, with space group (P-3m1). Figure 1 shows the $1 T$ geometry for a monolayered structures in the left side, the top and the side views of zigzag and armchair NTs are represented in the middle and the right parts, respectively. The optimized lattice parameters and bond lengths with respect to the tube diameter are shown in Figure 2. Increasing the tube diameter $d$, the lattice vectors of the tubes decrease for both zigzag and armchair chiralities. For $(n, n)$ NTs, the lattice vectors correspond to those of the MLs; however, due to the curvature they enlarge as the diameters become smaller. Similar behaviour is observed for the bond lengths between metal and chalcogen atoms $(M-X)$, nevertheless, the convergence to the ML limit is much slower. Generally, the bond lengths and lattice vectors of selenide NTs are larger than those of sulphide NTs, and the same holds for the comparison of the platinum over the palladium forms.

The $M-X$ bond lengths can be divided into two types, the inner and the outer wall bond lengths, which are referred hereafter as $M-X_{i}$ and $M-X_{o}$, respectively. The $M-X_{o}\left(M-X_{i}\right)$ are longer (shorter) than the corresponding bond lengths found in the ML structures, and the deviations are more pronounced for armchair NTs than for zigzag NTs, particularly for small $d$. For diameters below $15 \AA$, the difference between outer and inner bond reaches $0.40 \AA$ and $0.25 \AA$ for armchair and zigzag NTs, respectively. These numbers strongly reduce to about $0.10 \AA$ difference for diameters of at least $40 \AA$. Recently, we have reported that the bond lengths of $\mathrm{MoS}_{2}$ and $\mathrm{WS}_{2}$ NTs exhibit opposite behaviour, where the zigzag NTs have longer (shorter) $M-X_{o}\left(M-X_{i}\right)$ bond lengths than the armchair NTs [46].

Figure 1. 1T 2D (monolayer) and 1D (tubular) forms of noble metal chalcogenides. The unit cell of 2D systems is shown.
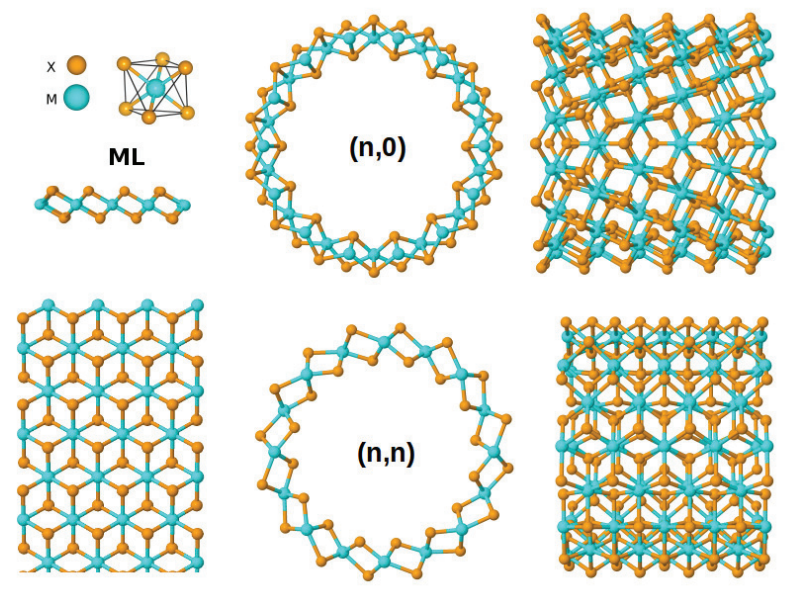
Figure 2. Lattice parameters and bond lengths $v s$. diameter of $M X_{2}$ NTs.
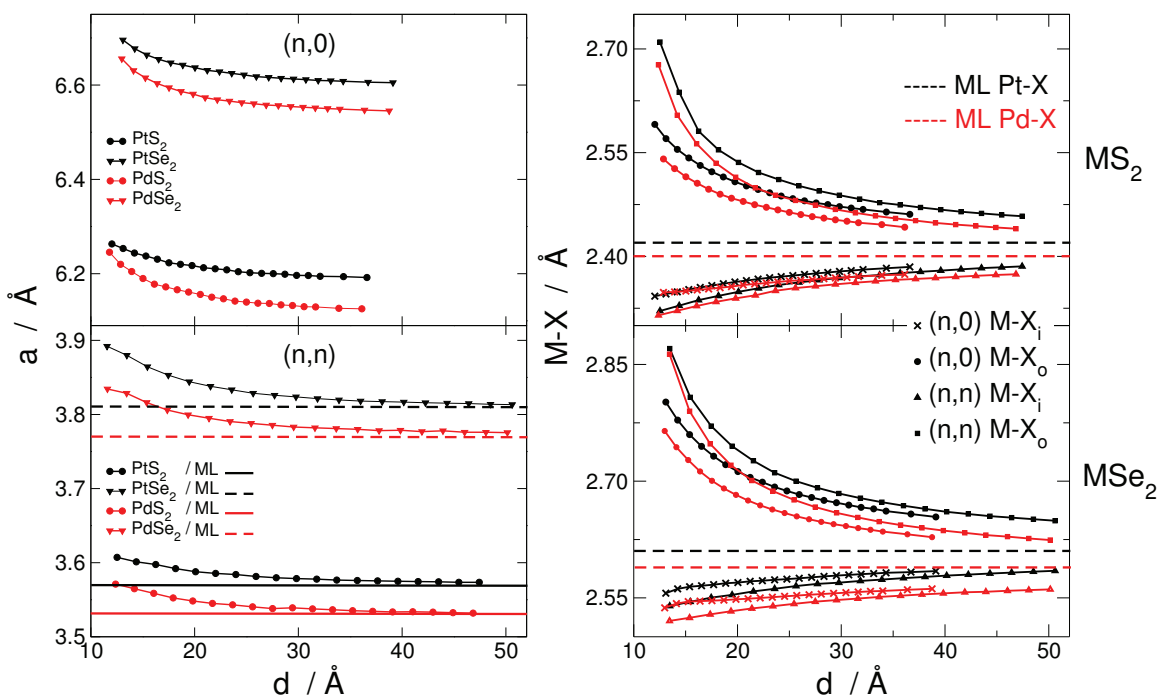

The stability of NTs can be expressed by the strain energy, $E_{\text {Strain }}$, which is the difference of total energy per atom of the tube and the respective ML. Generally, the strain energy of NTs is correlated to the tube diameter through the archetypal relation $E_{\text {Strain }} \sim 1 / d^{2}$. The calculated $E_{\text {Strain }}$ of $M X_{2}$ NTs with respect to their diameters (see Figure 3) follow the same dependence, where the strain energies decrease quadratically with the diameter and converge to the same value for all systems. The curves $E_{\text {Strain }}(d)$ were fitted to the equation $E_{\text {Strain }}=C / d^{2}$ with correlation coefficients greater than 0.999 and the values of coefficients $C$ are given in the Table 1 for each system. We note that strain energies of the $\mathrm{Pd} X_{2}$ NTs are smaller than for $\mathrm{Pt} X_{2}$ NTs for small diameters, this means that $\operatorname{Pd} X_{2}$ NTs are more stiff than $\mathrm{Pt} X_{2}$ NTs in that range. Furthermore, the strain energy of noble-metal chalcogenide tubes is the same for both zigzag and armchair chiralities, whereas for $\mathrm{MoS}_{2}$ and $\mathrm{WS}_{2}$ counterparts, armchair NTs are more stable than zigzag NTs, especially for large diameters [46]. In addition, the coefficients $C$ and the strain energy values of the noble-metal chalcogenide NTs are smaller than those of $\mathrm{MoS}_{2}$ and $\mathrm{WS}_{2}$ NTs. This means that noble-metal chalcogenide NTs are more favorable and easier to form than $\mathrm{MoS}_{2}$ and $\mathrm{WS}_{2} \mathrm{NTs}$.

Figure 3. Strain energy $v s$. diameter of $M X_{2}$ nanotubes.
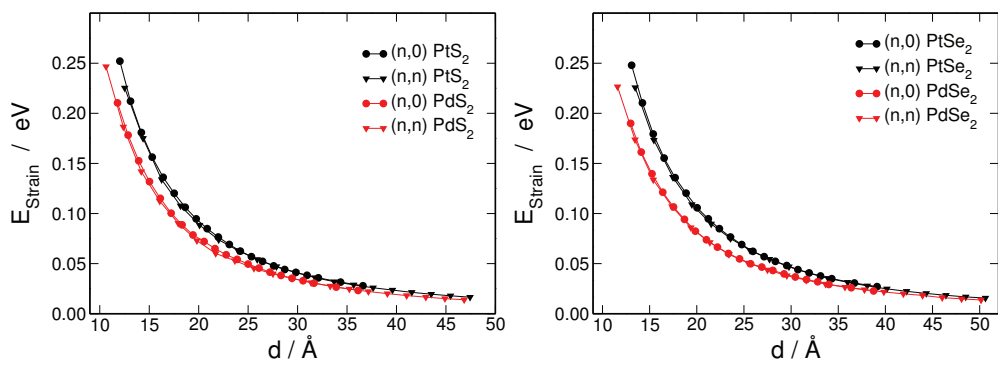
Table 1. Coefficients $C$ ( in $\mathrm{eV} \AA^{2}$ ) of the fitted curves $E_{\text {Strain }}=C / d^{2}$.

\begin{tabular}{ccc}
\hline System & Zigzag $(n, \mathbf{0})$ & Armchair $(n, n)$ \\
\hline $\mathrm{PtS}_{2}$ & 36.60 & 35.71 \\
$\mathrm{PtSe}_{2}$ & 42.47 & 41.22 \\
$\mathrm{PdS}_{2}$ & 29.71 & 28.46 \\
$\mathrm{PdSe}_{2}$ & 32.50 & 31.38 \\
$\mathrm{MoS}_{2}[46]$ & 57.50 & 50.90 \\
$\mathrm{WS}_{2}[46]$ & 58.14 & 59.68 \\
\hline
\end{tabular}

We have also investigated the electronic structure of these noble-metal chalcogenide systems. The $M X_{2}$ MLs are found to be semiconducting with indirect band gaps of $1.26 \mathrm{eV}, 1.75 \mathrm{eV}$, $0.74 \mathrm{eV}$ and $1.38 \mathrm{eV}$ for $\mathrm{PdS}_{2}, \mathrm{PtS}_{2}, \mathrm{PdSe}_{2}, \mathrm{PtSe}_{2}$, respectively. This is in agreement with the results of Miró et al. [39] for these $\mathrm{MX}_{2}$ MLs, where the obtained band gaps are $1.11 \mathrm{eV}, 1.75 \mathrm{eV}$, $0.39 \mathrm{eV}$ and $1.05 \mathrm{eV}$ for $\mathrm{PdS}_{2}, \mathrm{PtS}_{2}, \mathrm{PdSe}_{2}$ and $\mathrm{PtSe}_{2}$, respectively, using similar level of theory.

The band gaps $\Delta$ versus the tube diameter of all $M X_{2}$ NTs are plotted in Figure 4. Similar to $\mathrm{MoS}_{2}$ and $\mathrm{WS}_{2}$ NTs, these band gaps of $M X_{2}$ NTs increase with the diameter and approach the band gaps of their respective MLs. For small $d$, the band gaps of armchair NTs become larger than zigazag NTs for all systems. Substituting Pd with Pt causes an increase in $\Delta$, while replacing $\mathrm{S}$ by $\mathrm{Se}$ decreases it. The band structures of noble-metal $M X_{2}$ MLs and NTs are depicted in Figure 5. Unlike $\mathrm{MoS}_{2}$ and $\mathrm{WS}_{2}$ MLs, where the band gaps are direct with values of 1.9 and $2.1 \mathrm{eV}$, respectively [47], the noble-metal MLs are all indirect bandgap semiconductors. This difference could be understood in the electronic configuration of the metal elements, as well as, in the $2 \mathrm{H}$ and $1 T$ symmetries of $\mathrm{MoS}_{2} / \mathrm{WS}_{2}$ and the noble-metal chalcogenides, respectively. The $M X_{2}$ chalcogenide NTs also exhibit indirect band gaps, for both zigazags and armchairs and for all systems, whereas $\mathrm{MoS}_{2}$ and $\mathrm{WS}_{2}$ NTs show direct and indirect band gaps for zigzag and armchair forms, respectively [46].

Figure 4. Band gap vs. diameter of $M X_{2}$ nanotubes.
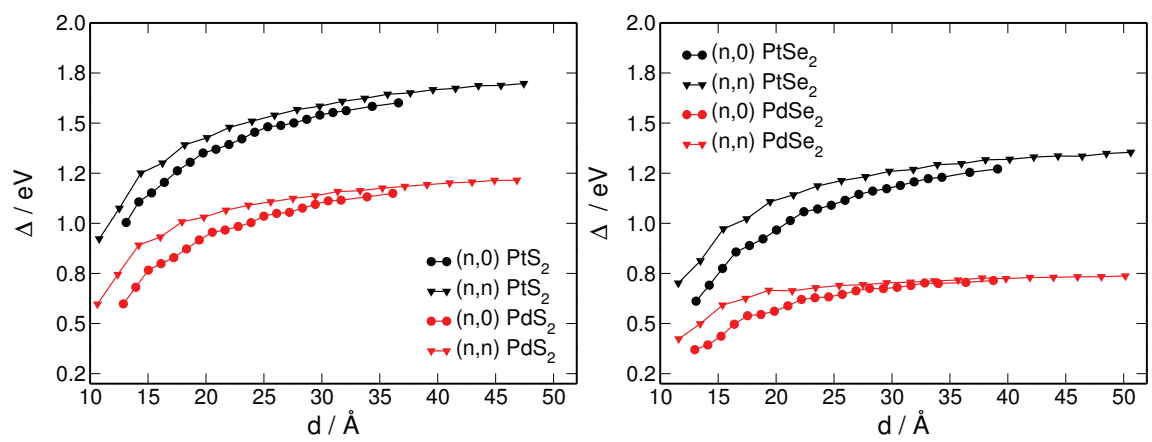
Figure 5. Band structures of monolayers, $(32,0)$, $(18,18)$ nanotubes of $M X_{2}$ systems, respectively.
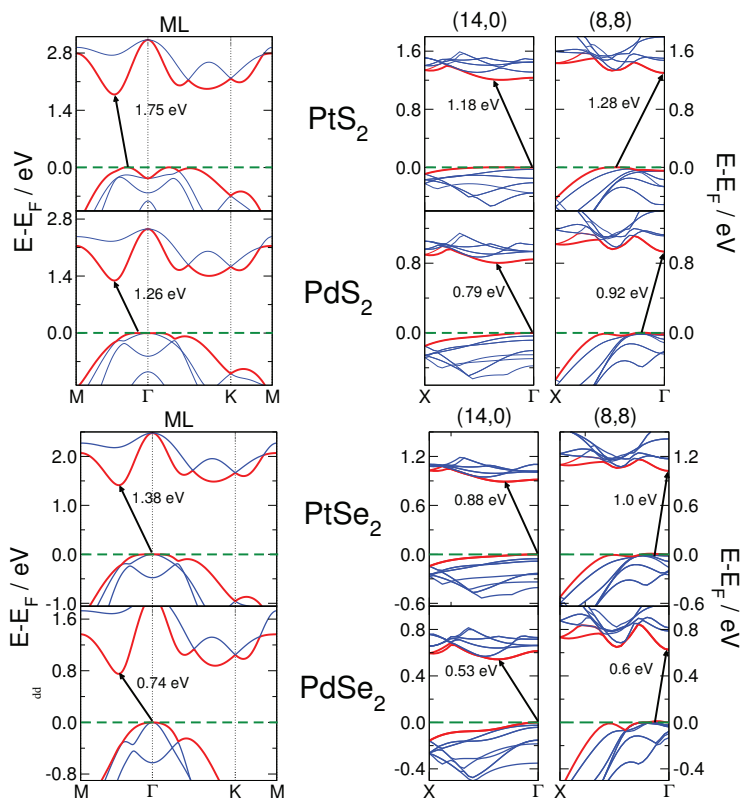

\section{Conclusions}

In analogy to the existing transition metal chalcogenide nanotubes, we have investigated hypothetical noble-metal chalcogenide nanotubes $\left(\mathrm{PdS}_{2}, \mathrm{PdSe}_{2}, \mathrm{PtS}_{2}\right.$ and $\left.\mathrm{PtSe}_{2} \mathrm{NTs}\right)$ through density functional theory calculations. We have shown that formation of these nanotubes is possible, since they have smaller strain energies than $\mathrm{MoS}_{2}$ or $\mathrm{WS}_{2}$ nanotubes. Furthermore, we have found that the strain energy of the studied nanotubes is chirality independent and decreases inverse quadratically with the tube diameter. Moreover, $\operatorname{Pd} X_{2}$ nanotubes are more stable than $\operatorname{Pt} X_{2}$ for nanotubes with small diameters. We have also examined the electronic structure of noble-metal chalcogenide monolayers and nanotubes, which are found to be all indirect band gap semiconductors in the ranges of 0.6-1.1 eV (0.9-1.7 eV) and 0.3-0.8 eV (0.6-1.3 eV) for $\mathrm{PdS}_{2}\left(\mathrm{PtS}_{2}\right)$ and $\mathrm{PdSe}_{2}\left(\mathrm{PtSe}_{2}\right) \mathrm{NTs}$, respectively. These NTs band gaps increase with the diameter rapidly approaching that of the respective pristine $2 \mathrm{D}$ monolayer.

\section{Acknowldgements}

This work was supported by the German Research Council (Deutsche Forschungsgemeinschaft, HE 3543/18-1), the European Commission (FP7-PEOPLE-2009-IAPP QUASINANO, GA 251149 and FP7-PEOPLE-2012-ITN MoWSeS, GA 317451). 


\section{Author Contributions}

Nourdine Zibouche, Agnieszka Kuc, Pere Miró and Thomas Heine generated, analyzed and discussed the results. Thomas Heine conceived this project. All authors contributed in writing this paper.

\section{Conflicts of Interest}

The authors declare no conflict of interest.

\section{References}

1. Pauling, L. The structure of the chlorites. Proc. Natl. Acad. Sci. USA 1930, 16, 578-582.

2. Iijima, S. Helical Microtubules of Graphitic Carbon. Nature 1991, 354, 56-58.

3. Tenne, R.; Margulis, L.; Genut, M.; Hodes, G. Polyhedral and Cylindrical Structures of Tungsten Disulfide. Nature 1992, 360, 444.

4. Margulis, L.; Salitra, G.; Tenne, R.; Talianker, M. Nested fullerene-like structures. Nature 1993, 365, 113-114.

5. Chopra, N.G.; Luyken, R.J.; Cherrey, K.; Crespi, V.H.; Cohen, M.L.; Louie, S.G.; Zettl, A. Boron Nitride Nanotubes. Science 1995, 269, 966-967.

6. Yin, L.W.; Bando, Y.; Golberg, D.; Li, M.S. Growth of Single-Crystal Indium Nitride Nanotubes and Nanowires by a Controlled-Carbonitridation Reaction Route. Adv. Mater. 2004, 16, 1833-1838.

7. Spahr, M.; Bitterli, P.; Nesper, R.; Mueller, M.; Krumeich, F.; Nissen, H.U. Redoxaktive Nanoröhren aus Vanadiumoxid. Angew. Chem. 1998, 110, 1339-1342.

8. Kasuga, T.; Hiramatsu, M.; Hoson, A.; Sekino, T.; Niihara, K. Titania Nanotubes Prepared by Chemical Processing. Adv. Mater. 1999, 11, 1307-1311.

9. Yada, M.; Mihara, M.; Mouri, S.; Kuroki, M.; Kijima, T. Rare Earth (Er, Tm, Yb, Lu) Oxide Nanotubes Templated by Dodecylsulfate Assemblies. Adv. Mater. 2002, 14, 309-313.

10. Radovsky, G.; Popovitz-Biro, R.; Stroppa, D.G.; Houben, L.; Tenne, R. Nanotubes from Chalcogenide Misfit Compounds: $\mathrm{Sn}-\mathrm{S}$ and Nb-Pb-S. Acc. Chem. Res. 2014, 47, 406-416.

11. Zhu, Y.Q.; Sekine, T.; Brigatti, K.S.; Firth, S.; Tenne, R.; Rosentsveig, R.; Kroto, H.W.; Walton, D.R.M. Shock-Wave Resistance of $\mathrm{WS}_{2}$ Nanotubes. J. Am. Chem. Soc. 2003, 125, 1329-1333.

12. Kaplan-Ashiri, I.; Cohen, S.R.; Gartsman, K.; Rosentsveig, R.; Ivanovskaya, V.; Heine, T.; Seifert, G.; Wagner, H.D.; Tenne, R. Mechanical properties of individual $\mathrm{WS}_{2}$ nanotubes. Electron. Prop. Synth. Nanostruct. 2004, 723, 306-312.

13. Kaplan-Ashiri, I.; Cohen, S.R.; Gartsman, K.; Rosentsveig, R.; Seifert, G.; Tenne, R. Mechanical behavior of individual $\mathrm{WS}_{2}$ nanotubes. J. Mater. Res. 2004, 19, 454-459.

14. Kaplan-Ashiri, I.; Cohen, S.R.; Gartsman, K.; Rosentsveig, R.; Ivanovskaya, V.; Heine, T.; Seifert, G.; Wagner, H.D.; Tenne, R. Direct tensile tests of individual $\mathrm{WS}_{2}$ nanotubes. Mater. Sci. Forum 2005, 475-479, 4097-4102. 
15. Kaplan-Ashiri, I.; Cohen, S.R.; Gartsman, K.; Ivanovskaya, V.V.; Heine, T.; Seifert, G.; Wiesel, I.; Wagner, H.D.; Tenne, R. On the Mechanical Behavior of $\mathrm{WS}_{2}$ Nanotubes under Axial Tension and Compression. Proc. Natl. Acad. Sci. USA 2006, 103, 523-528.

16. Kaplan-Ashiri, I.; Tenne, R. Mechanical Properties of $\mathrm{WS}_{2}$ Nanotubes. J. Clust. Sci. 2007, 18, 549-563.

17. Rapoport, L.; Bilik, Y.; Feldman, Y.; Homyonfer, M.; Cohen, S.R.; Tenne, R. Hollow nanoparticles of $\mathrm{WS}_{2}$ as potential solid-state lubricants. Nature 1997, 387, 791-793.

18. Rothschild, A.; Cohen, S.R.; Tenne, R. $\mathrm{WS}_{2}$ nanotubes as tips in scanning probe microscopy. Appl. Phys. Lett. 1999, 75, 4025, doi:10.1063/1.125526.

19. Komarneni, M.R.; Yu, Z.; Burghaus, U.; Tsverin, Y.; Zak, A.; Feldman, Y.; Tenne, R. Characterization of Ni-Coated $\mathrm{WS}_{2}$ Nanotubes for Hydrodesulfurization Catalysis. Isr. J. Chem. 2012, 52, 1053-1062.

20. Zhang, W.; Ge, S.; Wang, Y.; Rafailovich, M.H.; Dhez, O.; Winesett, D.A.; Ade, H.; Shafi, K.V.; Ulman, A.; Popovitz-Biro, R. Use of functionalized $\mathrm{WS}_{2}$ nanotubes to produce new polystyrene/polymethylmethacrylate nanocomposites. Polymer 2003, 44, 2109-2115.

21. Unalan, H.E.; Yang, Y.; Zhang, Y.; Hiralal, P.; Kuo, D.; Dalal, S.; Butler, T.; Cha, S.N.; Jang, J.E.; Chremmou, K.; et al. ZnO Nanowire and $\mathrm{WS}_{2}$ Nanotube Electronics. IEEE Trans. Electron Devices 2008, 55, 2988-3000.

22. Wang, G.X.; Bewlay, S.; Yao, J.; Liu, H.K.; Dou, S.X. Tungsten Disulfide Nanotubes for Lithium Storage. Electrochem. Solid-State Lett. 2004, 7, A321-A323.

23. Hua, M.; Zhanliang, T.; Feng, G.; Jun, C.; Huatang, Y. Synthesis, characterization and hydrogen storage capacity of $\mathrm{MS}_{2}(M=\mathrm{Mo}$, Ti) nanotubes. Front. Chem. China 2006, 1, 260-263.

24. Chen, J.; Li, S.L.; Tao, Z.L.; Gao, F. Low-temperature synthesis of titanium disulfide nanotubes. Chem. Commun. 2003, 980-981.

25. Remskar, M.; Mrzel, A.; Jesih, A.; Leperevy, F. Metal-Alloyed $\mathrm{NbS}_{2}$ Nanotubes Synthesized by the Self-Assembly of Nanoparticles. Adv. Mater. 2002, 14, 680-684.

26. Coleman, K.S.; Sloan, J.; Hanson, N.A.; Brown, G.; Clancy, G.P.; Terrones, M.; Terrones, H.; Green, M.L.H. The Formation of $\mathrm{ReS}_{2}$ Inorganic Fullerene-like Structures Containing Re4 Parallelogram Units and Metal-Metal Bonds. J. Am. Chem. Soc. 2002, 124, 11580-11581.

27. Chen, J.; Tao, Z.L.; Li, S.L.; Fan, X.B.; Chou, S.L. Synthesis of $\mathrm{TiSe}_{2}$ Nanotubes/Nanowires. Adv. Mater. 2003, 15, 1379-1382.

28. Li, P.; Stender, C.L.; Ringe, E.; Marks, L.D.; Odom, T.W. Synthesis of $\mathrm{TaS}_{2}$ Nanotubes from $\mathrm{Ta}_{2} \mathrm{O}_{5}$ Nanotube Templates. Small 2010, 6, 1096-1099.

29. Miro, P.; Audiffred, M.; Heine, T. An Atlas of Two-Dimensional Materials. Chem. Soc. Rev. 2014, 43, 6537-6554.

30. Nicolosi, V.; Chhowalla, M.; Kanatzidis, M.G.; Strano, M.S.; Coleman, J.N. Liquid Exfoliation of Layered Materials. Science 2013, 340, doi:10.1126/science.1226419.

31. Remskar, M.; Skraba, Z.; Regula, M.; Ballif, C.; Sanjines, R.; Levy, F. New crystal structures of $\mathrm{WS}_{2}$ : Microtubes, ribbons, and ropes. Adv. Mater. 1998, 10, 246-249. 
32. Catherine, M.; Zelenski, P.K.D. Template Synthesis of Near-Monodisperse Microscale Nanofibers and Nanotubules of $\mathrm{MoS}_{2}$. J. Am. Chem. Soc. 1998, 120, 734-742.

33. Zhu, Y.Q.; Hsu, W.K.; Grobert, N.; Chang, B.H.; Terrones, M.; Terrones, H.; Kroto, H.W.; Walton, D.R.M. Production of $\mathrm{WS}_{2}$ Nanotubes. Chem. Mater 2000, 12, 1190-1194.

34. Feldman, Y.; Wasserman, E.; Srolovitz, D.J.; Tenne, R. High-Rate, Gas-Phase Growth of $\mathrm{MoS}_{2}$ Nested Inorganic Fullerenes and Nanotubes. Science 1995, 267, 222-225.

35. Gronvold, F.; Rost, E. On the sulfides, selenides and tellurides of palladium. Acta Chem. Scand. 1956, 10, 1620-1634.

36. Kjekshus, A.; Gronvold, F. High temperature X-ray study of the thermal expansionn of $\mathrm{PtS}_{2}$, $\mathrm{PtSe}_{2}, \mathrm{PtTe}_{2}$ and $\mathrm{PdTe}_{2}$. Acta Chem. Scand. 1959, 13, 1767-1774.

37. Gronvold, F.; Haraldsen, H.; Kjekshus, A. On the sulfides, selenides and tellurides of platinium. Acta Chem. Scand. 1960, 14, 1879-1893.

38. Wilson, J.A.; Yoffe, A.D. The Transition Metal Dichalcogenides Discussion and Interpretation of Observed Optical, Electrical and Structural Properties. Adv. Phys. 1969, 18, 193-335.

39. Miro, P.; Ghorbani-Asl, M.; Heine, T. Two Dimensional Materials Beyond $\mathrm{MoS}_{2}$ : Noble-Transition-Metal Dichalcogenides. Angew. Chem. Int. Ed. 2014, 53, 3015-3018.

40. Perdew, J.P.; Burke, K.; Ernzerhof, M. Generalized gradient approximation made simple. Phys. Rev. Lett. 1996, 77, 3865, doi:10.1103/PhysRevLett.77.3865.

41. Dovesi, R.; Saunders, V.R.; Roetti, R.; Orlando, R.; Zicovich-Wilson, C.M.; Pascale, F.; Civalleri, B.; Doll, K.; Harrison, N.M.; Bush, I.J.; et al. CRYSTAL09 User's Manual; University of Torino: Torino, Italy, 2009.

42. Peintinger, M.F.; Oliveira, D.V.; Bredow, T. Consistent Gaussian basis sets of triple-zeta valence with polarization quality for solid-state calculations. J. Comput. Chem. 2013, 34, 451-459.

43. Doll, K. Adsorption on the Pt surface a comparison of a gradient corrected functional and a hybrid functional. Surf. Sci. 2004, 573, $464-473$.

44. Stoll, H.; Metz, B.; Dolg, M. Relativistic energy-consistent pseudopotentials-recent developments. J. Comput. Chem. 2002, 23, 767-778.

45. Monkhorst, H.J.; Pack, J.D. Special Points For Brillouin-Zone Integrations. Phys. Rev. B 1976, 13, 5188, doi:10.1103/PhysRevB.13.5188.

46. Zibouche, N.; Kuc, A.; Heine, T. From layers to nanotubes: Transition metal disulfides $\mathrm{TMS}_{2}$. Eur. Phys. J. B 2012, 85, 49, doi:10.1140/epjb/e2011-20442-1.

47. Kuc, A.; Zibouche, N.; Heine, T. Influence of quantum confinement on the electronic structure of the transition metal sulfide $\mathrm{TS}_{2}$. Phys. Rev. B 2011, 83, doi:10.1103/PhysRevB.83.245213. 
MDPI AG

Klybeckstrasse 64

4057 Basel, Switzerland

Tel. +41 616837734

Fax +4161302 8918

http://www.mdpi.com/

Inorganics Editorial Office

E-mail: inorganics@mdpi.com

http://www.mdpi.com/journal/inorganics 

口觜口 $\mathrm{MDPI}$ - Basel • Beijing • Wuhan ISBN 978-3-03842-149-8 www.mdpi.com 